Portland State University

PDXScholar

Summer 7-25-2014

\title{
Exploring Pedestrian Responsive Traffic Signal Timing Strategies in Urban Areas
}

Sirisha Murthy Kothuri

Portland State University

Follow this and additional works at: https://pdxscholar.library.pdx.edu/open_access_etds

Part of the Transportation Engineering Commons

Let us know how access to this document benefits you.

\section{Recommended Citation}

Kothuri, Sirisha Murthy, "Exploring Pedestrian Responsive Traffic Signal Timing Strategies in Urban Areas" (2014). Dissertations and Theses. Paper 1934.

https://doi.org/10.15760/etd.1933

This Dissertation is brought to you for free and open access. It has been accepted for inclusion in Dissertations and Theses by an authorized administrator of PDXScholar. Please contact us if we can make this document more accessible: pdxscholar@pdx.edu. 
Exploring Pedestrian Responsive Traffic Signal Timing Strategies In Urban Areas

by

Sirisha Murthy Kothuri

A dissertation submitted in partial fulfillment of the requirements for the degree of

\author{
Doctor of Philosophy \\ in \\ Civil and Environmental Engineering
}

Dissertation Committee:

Christopher Monsere, Chair

Kelly Clifton

Robert L. Bertini

Robert Fountain

Portland State University

2014 
C 2014 Sirisha Murthy Kothuri 


\begin{abstract}
The role of walking in the development of healthy, livable communities is being increasingly recognized. In urban areas, intersections represent locations where different modes converge, and are often viewed as deterrents to walking. This is due to the unwarranted and often unnecessary delays imposed by signal timing policies for pedestrians and increased potential for conflicts. Traditional signal timing design prioritizes vehicles over pedestrians leading to undesirable consequences such as large delays and risky pedestrian behaviors. Pedestrians are accommodated in a manner that is designed to cause least interruption to the flow of motor vehicles. This lack of pedestrian accommodation at signalized intersections is the focus of this dissertation.

Understanding pedestrian attitudes and perceptions is important because it offers insights into actual crossing behavior at signalized intersections. An intercept survey of 367 crossing pedestrians was undertaken at four signalized intersections in Portland, Oregon, and binary logistic regression models were constructed to quantify the impacts of demographics, trip characteristics and type of infrastructure on pedestrian perceptions and attitudes regarding delay, crossing time and motivators for crossing decisions. Safety was found to have a larger effect than compliance on the decision to cross the street. Pedestrians at recall intersections expressed higher satisfaction with delay than at actuated intersections.
\end{abstract}


Novel methods to measure pedestrian delay using 2070 signal controllers and Voyage software were developed. These methods have been adopted by the City of Portland to record actuation trends and delays at various intersections. In the absence of demand data, pedestrian push button actuations can be considered as a proxy for crossing demand. The micro-simulation software VISSIM was used to analyze delays resulting from varying pedestrian and vehicle volumes on a network of three intersections in Portland, Oregon. From a pedestrian perspective, free operation was found to be always beneficial due to lower pedestrian delays. However, from a system wide perspective, free operation was found to be beneficial only under low-medium traffic conditions from an overall delay reduction viewpoint, while coordinated operation showed benefits under heavy traffic conditions, irrespective of the volume of pedestrians. Control strategies were developed to identify the best mode of signal controller operation that produced the lowest overall average delay per user. A procedure to identify the optimal control strategy based on user inputs (major street volume to capacity ratios and rate of pedestrian phase serviced for the minor street) was developed. The procedure was applied to a network of three intersections in east Portland, OR and the findings were verified.

This research offers significant contributions in the field of pedestrian research. The findings related to attitudes and perceptions of crossing pedestrians offer greater insights into pedestrian crossing behavior and add to the body of existing literature. The methods developed to obtain pedestrian actuations and delay data from signal controllers represent an easy and cost-effective way to characterize pedestrian service at intersections. The results pertaining to signal timing strategies represent an important step 
towards incorporating pedestrian needs at intersections and demonstrate how control strategies employed to benefit pedestrians could benefit the entire system. 


\section{DEDICATION}

To my family 


\section{ACKNOWLEDGEMENTS}

Along this journey, there have been many people who have supported and encouraged me. I would like to express my deep appreciation and gratitude to my advisor Dr. Chris Monsere for his patience and guidance during the course of my research. I would also like to give special thanks to Dr. Robert Bertini, who encouraged me to start this journey many years ago and for his untiring support throughout. I would like to thank Dr. Kelly Clifton for motivating me constantly and for serving on my committee. Thanks are also due to Dr. Robert Fountain for participating on my committee.

I would also like to express my heartfelt gratitude to Peter Koonce and Ty Reynolds who served as my mentors and provided ample guidance. I would also like to recognize Miranda Wells, Alex Kiheri and the PTV support staff for their help with VISSIM software. To all past and present ITS lab members, your friendship and support along the way has been much appreciated. I also want to express my appreciation for the $\mathrm{PhT}$ group who provided critical feedback. I would also like to thank the staff in the Department of Civil Engineering as well as OTREC/NITC staff, for the administrative help. Thanks are also due to OTREC and NITC for funding this research.

Finally I'd like to express my profound gratitude to my family - my husband, son, parents, brothers and parents-in law for their constant encouragement. I could not have done it without your support and it is to you that I dedicate this work. 


\section{TABLE OF CONTENTS}

ABSTRACT

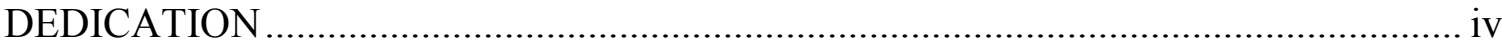

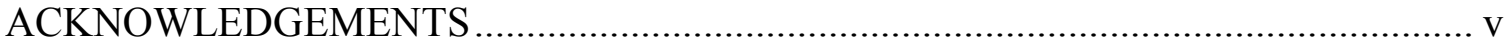

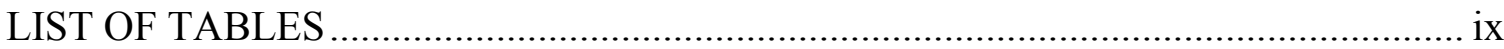

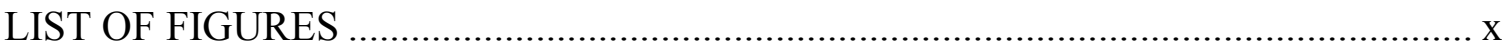

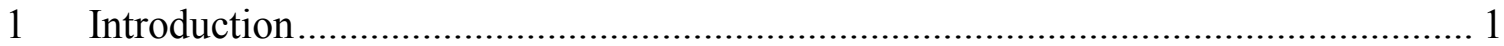

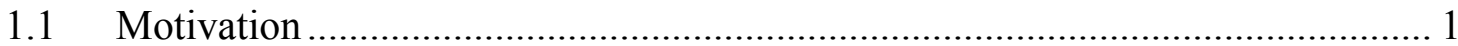

1.2 Research Goals and Objectives ............................................................... 2

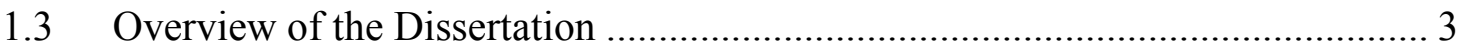

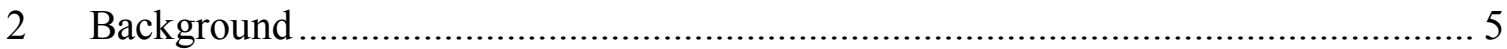

2.1 Signal Timing .......................................................................................... 5

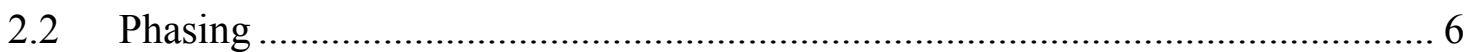

2.3 Pedestrian Signal Timing Schemes ............................................................. 7

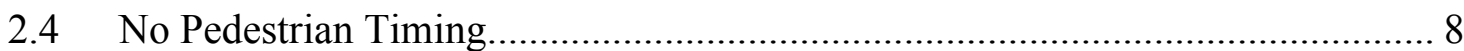

2.5 Concurrent Pedestrian Signal Timing ........................................................... 9

2.6 Leading Pedestrian Interval..................................................................... 10

2.6.1 Exclusive Pedestrian Phase ................................................................... 11

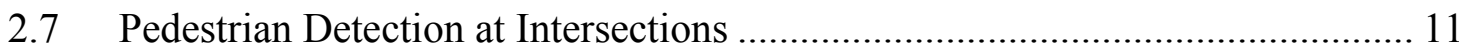

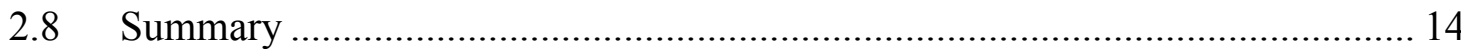

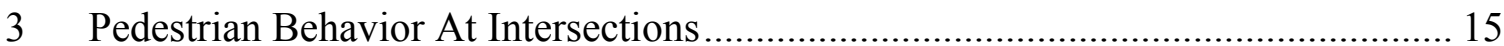

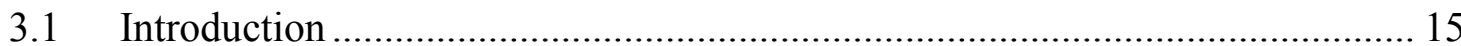

3.2 Background and Literature Review.......................................................... 16

3.2.1 Signal Violations and Compliance..................................................... 17

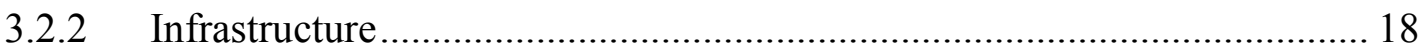

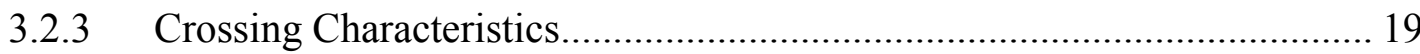

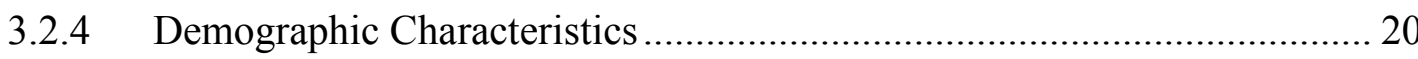

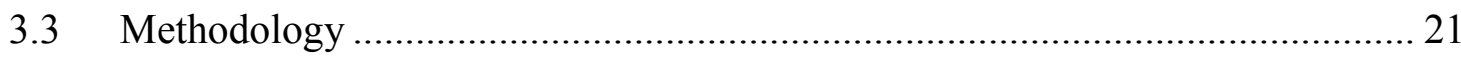

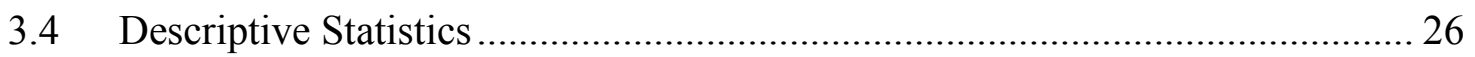

3.5 Model Development and Results ................................................................. 32

3.6 Discussion and Summary ...................................................................... 40 


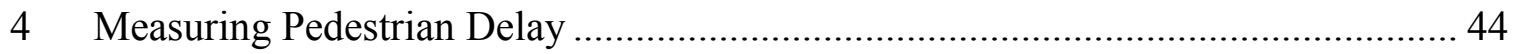

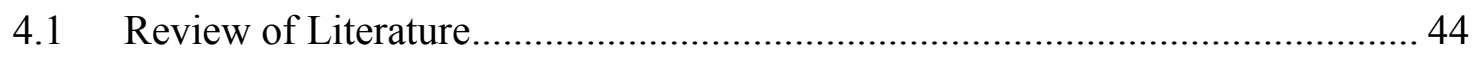

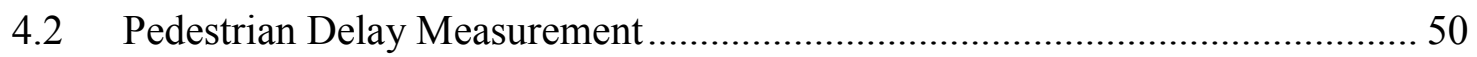

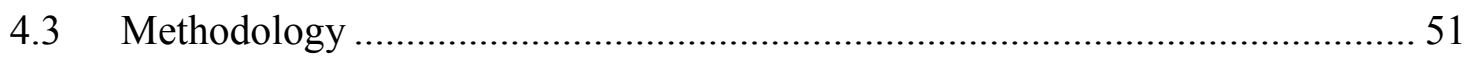

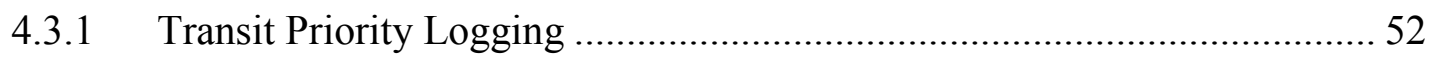

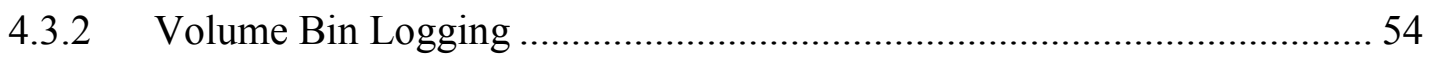

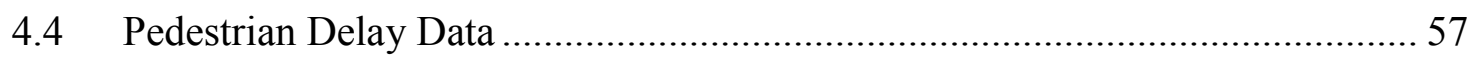

4.5 Field Test of Delay Effects of Control Strategies to Favor Pedestrians.............. 64

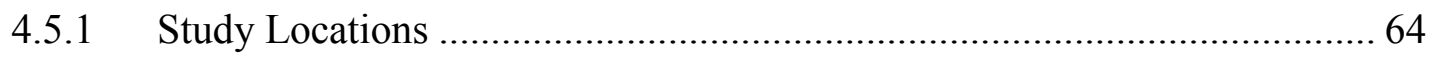

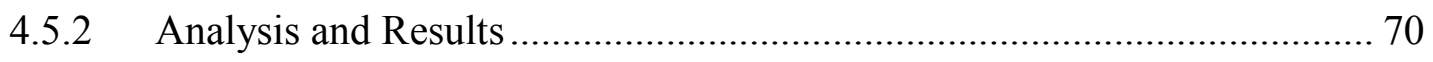

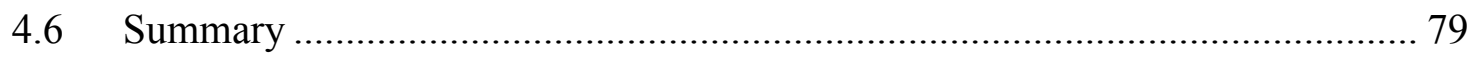

5 Exploring Pedestrian timing strateGies through Simulation Modeling .................... 81

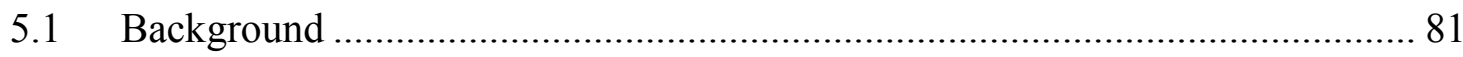

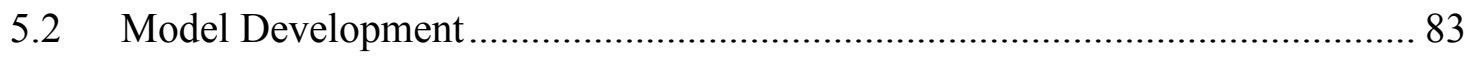

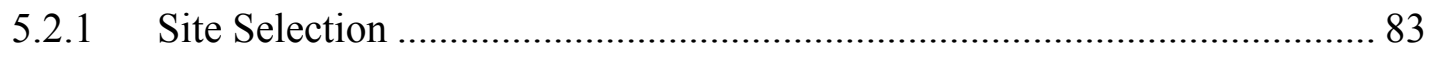

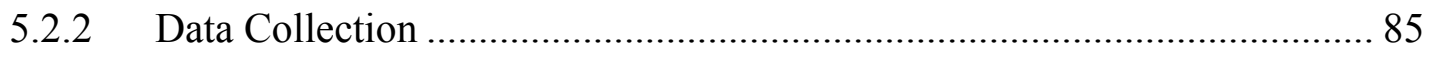

5.2.3 Network Development ........................................................................ 91

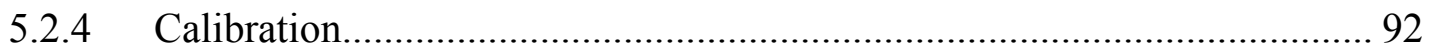

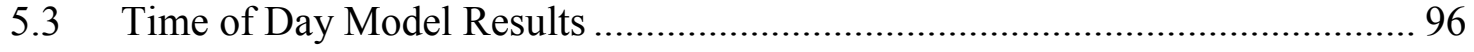

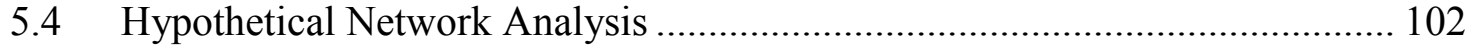

5.4.1 Hypothetical Network ........................................................................ 102

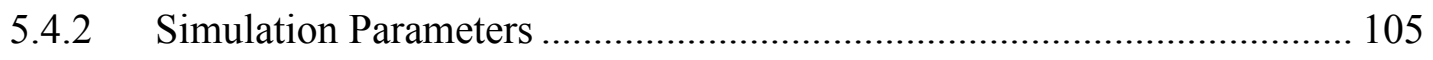

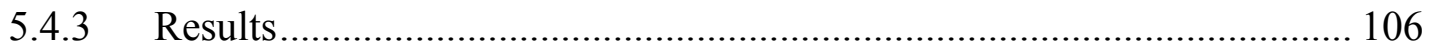

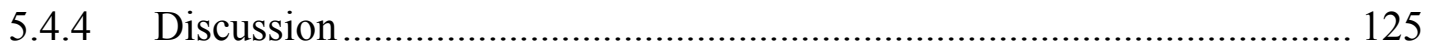

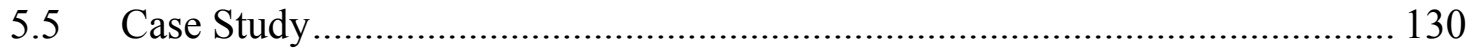

5.5.1 Data Inputs for Simulation................................................................ 131

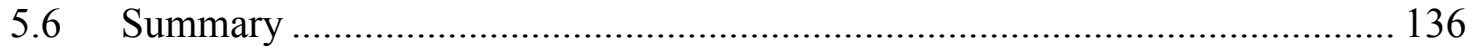

6 Conclusions and Recommendations ……………........................................... 138

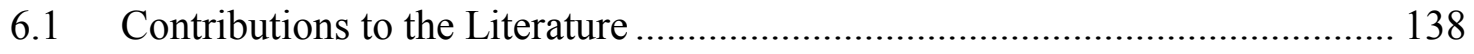

6.1.1 Signal Controller Data Logging ............................................................ 140

6.1.2 Pedestrian Crossing Behavior .............................................................. 141

6.1.3 Pedestrian Responsive Signal Timing Strategies..................................... 144 
6.2 Implications and Recommendations .................................................... 146

6.2.1 Using Existing Resources to Leverage Performance Measurement ......... 146

6.2.2 Signal Timing for all Users.................................................................. 147

6.2.3 The Three E's for Pedestrian Safety ....................................................... 149

6.3 Areas for Future Research.................................................................... 150

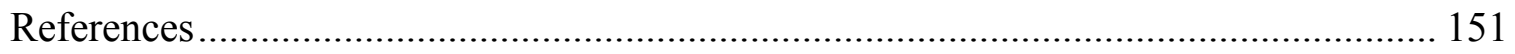

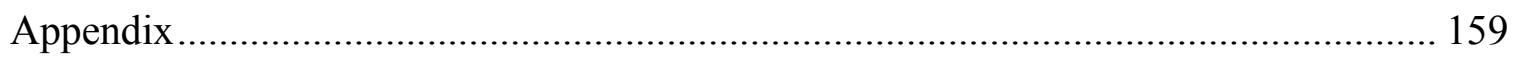




\section{LIST OF TABLES}

Table 3-1 Survey Questions..................................................................................... 23

Table 3-2 Summary Survey Response Statistics by Intersection.................................. 26

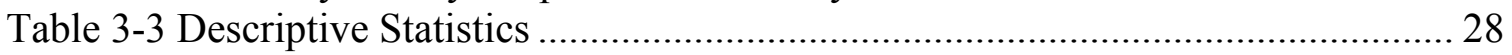

Table 3-4 Trip Characteristics by Intersection............................................................ 29

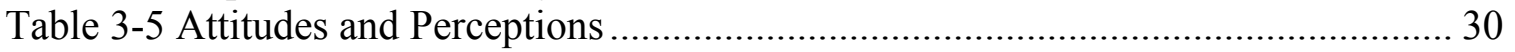

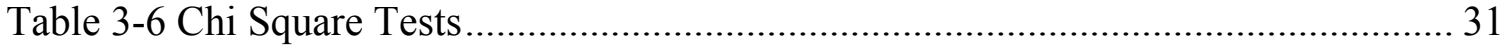

Table 3-7 Models of Influences on Crossing Decisions ............................................. 37

Table 3-8 Models of Delay Satisfaction in Actuated and Recall Intersections ................ 38

Table 3-9 Corridor Level Models of Satisfaction with Delay ...................................... 39

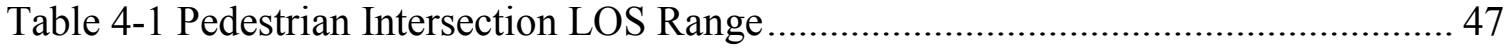

Table 4-2 Transit Priority Log with Pedestrian Delay Data .......................................... 58

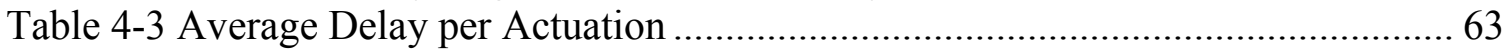

Table 4-4 Descriptive Statistics for Different Scenarios .............................................. 76

Table 4-5 Post hoc Tests for Different Scenarios ............................................................. 76

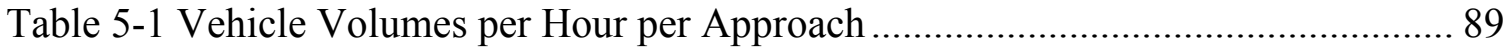

Table 5-2 Bicycle Volumes on Multnomah Boulevard ................................................ 90

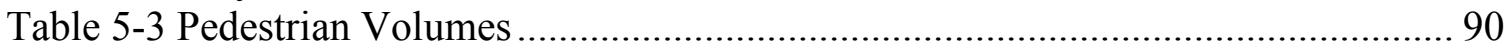

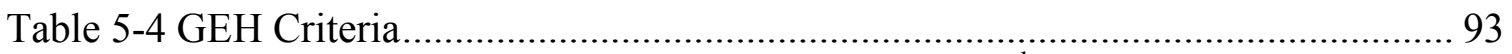

Table 5-5 GEH Statistic Calculations for Multnomah and $11^{\text {th }}$ using Mid-day Model .... 94

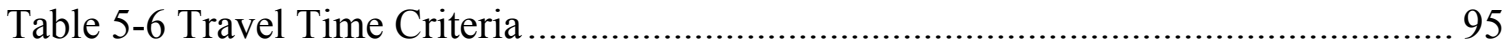

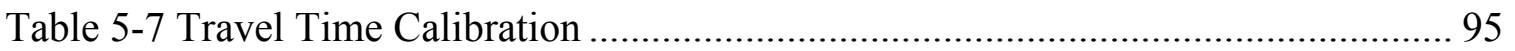

Table 5-8 Queue Lengths for Through Movements during Mid-day .............................. 96

Table 5-9 Performance Measures by Time of Day ........................................................ 99

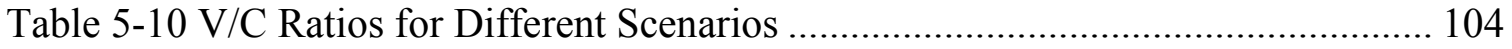

Table 5-11 Pedestrian Flows for Different Scenarios ................................................. 105

Table 5-12 Performance Metrics for Low Auto and Varying Pedestrian Volumes ....... 108

Table 5-13 Performance Metrics for Medium Auto and Varying Pedestrian Volumes . 110

Table 5-14 Performance Metrics for High Auto and Varying Pedestrian Volumes....... 111

Table 5-15 Average Delay Differences between Modes during Free operation and

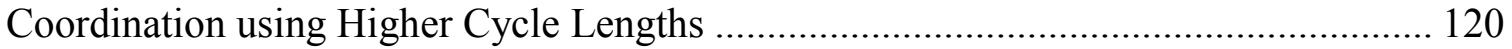

Table 5-16 Differences in Delay between Free and Fully Actuated Free Operation...... 125 


\section{LIST OF FIGURES}

Figure 2-1 Vehicle and Pedestrian Phases at an Intersection ........................................... 6

Figure 2-2 NEMA Ring and Barrier Diagram ............................................................. 7

Figure 2-3 Pedestrian Signal Displays with and without Countdown Timers.................... 8

Figure 2-4 Concurrent Pedestrian Timing .................................................................. 9

Figure 2-5 Leading Pedestrian Interval................................................................. 10

Figure 2-6 Exclusive Pedestrian Phase ..................................................................... 11

Figure 2-7 Pedestrian Push Buttons......................................................................... 13

Figure 3-1 Pedestrian Fatality Trends (2003-2012) ................................................... 16

Figure 3-2 Survey Locations...................................................................................... 25

Figure 4-1 Phase Diagram for NE 82nd Avenue and NE Tillamook Street..................... 52

Figure 4-2 Pedestrian Actuation Delay using Transit Priority Logs.................................. 53

Figure 4-3 Pedestrian Delay using Volume Logs ............................................................ 55

Figure 4-4 Map of Intersections with Actuation and Delay Data ................................... 59

Figure 4-5 Pedestrian Activity across Intersections on Monday, July 18, 2011 .............. 60

Figure 4-6 Pedestrian Actuations at SE 82nd and SE Division (July 15 -21, 2011) ........ 60

Figure 4-7 Pedestrian Actuations by Phase at SE 82 ${ }^{\text {nd }}$ and SE Division on July 19, 2011

Figure 4-8 NE 33rd Avenue and NE Hancock Street ......................................................... 65

Figure 4-9 Phase Diagram for NE 33rd Avenue and NE Hancock Street ......................... 66

Figure 4-10 Coordination Schedule and Time of Day Plans ............................................. 66

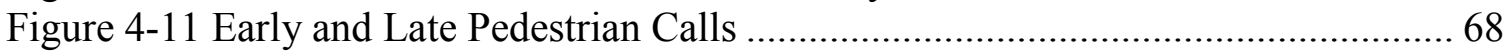

Figure 4-12 NE Sandy Boulevard and NE 16th Avenue ................................................ 69

Figure 4-13 Phase Diagram for NE Sandy Boulevard and NE 16th Avenue ................... 70

Figure 4-14 Late Pedestrian Calls for Permissive Length of $28 \mathrm{~s}$ and $35 \mathrm{~s}$..................... 73

Figure 4-15 Pedestrian Delay vs. Time of Actuation ................................................... 74

Figure 4-16 Box Plots of Pedestrian Delay................................................................ 75

Figure 4-17 Pedestrian Delay vs. Cycle Length ........................................................ 77

Figure 4-18 Distribution of Binned Delay ................................................................ 78

Figure 5-1 Intersections Chosen for Simulation ........................................................... 84

Figure 5-2 NE Multnomah Street Old Cross-section......................................................... 85

Figure 5-3 NE Multnomah Street New Cross-section with Cycle Track ......................... 85

Figure 5-4 Bicycle and Vehicle Count Locations............................................................ 86

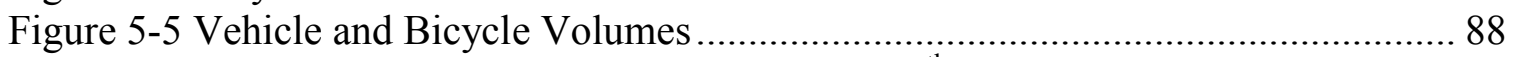

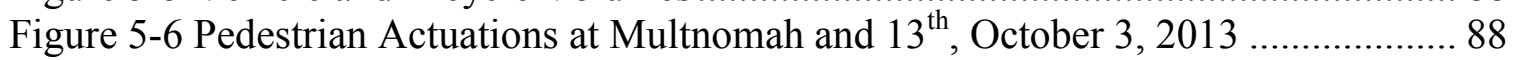

Figure 5-7 VISSIM Network for Multnomah Street ................................................. 92

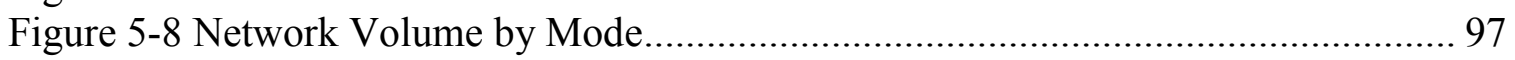

Figure 5-9 Average Delays per Person by Mode............................................................ 98

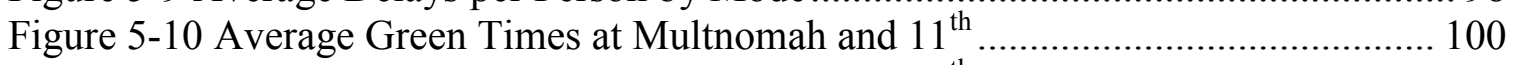

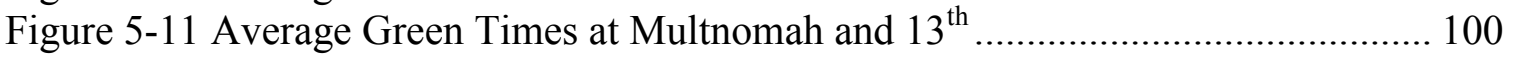




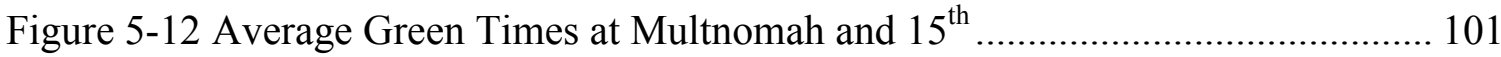

Figure 5-13 Auto, Bicycle and Pedestrian Volumes in the High Scenario.................... 105

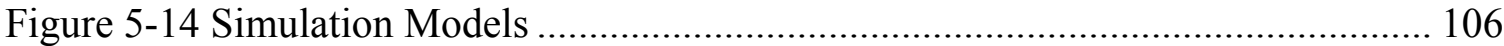

Figure 5-15 Actuated Pedestrian and Coordinated Movement Delay for Low Auto-Low

Ped Scenario

Figure 5-16 Actuated Pedestrian and Coordinated Movement Delay for Low Auto-High

Ped Scenario 114

Figure 5-17 Actuated Pedestrian and Coordinated Movement Delay for the High AutoLow Ped Scenario 115

Figure 5-18 Actuated Pedestrian and Coordinated Through Movement Delays for High Auto-High Ped Scenario 116

Figure 5-19 Comparison of Average Delays per User across Scenarios 117

Figure 5-20 Comparison of Average Delay per User between Coordinated (Optimized) and Free Operation 118

Figure 5-21 Percent Change in Average Delay per Person using 120s Cycle Length ... 120 Figure 5-22 Comparison of Modal Delays across Cycle Lengths and Free Operation .. 122 Figure 5-23 Comparison of Average Delays during Coordination and Fully Actuated Free Operation. 123

Figure 5-24 Strategy for Changing Signal Controller Mode of Operation .................... 127

Figure 5-25 Concept Mode of Operation based on Time of Day ................................ 128

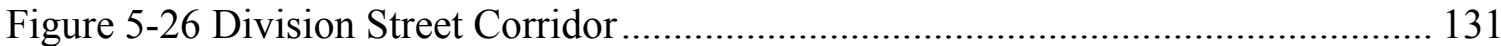

Figure 5-27 Division Street Network in VISSIM ..................................................... 132

Figure 5-28 Division Street Network Volumes ........................................................ 133

Figure 5-29 East and Westbound Bandwidths for Progression on Division Street ........ 134 Figure 5-30 Average Delay Comparison between Coordinated and Fully Actuated Free Modes on Division Street Network. 135

Figure 5-31 Percent Change in Average Delays on Division Street Network. 136 


\section{INTRODUCTION}

\subsection{Motivation}

Walking is a critical component in the development of healthy and sustainable communities. In the United States, walking trips have increased from 18 billion in 1990 to 42.5 billion in 2009; accounting for $10.9 \%$ of all trips undertaken (Santos, 2009). A nationwide survey of pedestrian and bicyclist attitudes found that the majority of walking trips were short- $67 \%$ of the trips were less than one mile in length (NHTSA, 2002). Many of these walking trips are in urban areas and require many street crossings, mostly at mid-block or intersections. Though generally viewed as the preferred place to cross the street for safety reasons, intersections can be a deterrent for walking if design and operation heavily favor motor vehicles.

When vehicle volumes are high, a traffic signal is used to separate conflicting users in time. Traditionally in the U.S., signal timing objectives have prioritized vehicle movements at intersections, often leading to large and unnecessary delays for pedestrians. In this thesis, pedestrian delay is defined as the difference between the time when a pedestrian activates the push button and the time that the pedestrian phase is served. Since most walking trips are short, the delays imposed by signal timing policies on pedestrians affect them disproportionately compared to other users. However, there is emerging interest within cities to actively promote active transportation modes, and to design and operate streets and intersections to accommodate all users efficiently. 
However, currently there is very limited research on accommodating and/or prioritizing pedestrians at signalized intersections in the North American context. Pedestrians are often considered as a deterrent to efficient vehicular traffic flow and therefore active efforts to include them in operational decisions at intersections have been lagging.

This research aims to fill that gap by understanding factors that influence pedestrian crossing behavior at signalized intersections and developing cost effective and easily deployable signal timing strategies that could be employed at intersections, to increase efficiency for pedestrians and also potentially improve compliance. The outcomes from this research offer insights into pedestrian behavior at intersections, which are critical for the design of safe facilities. In addition, the findings allow engineers and system operators to make better operational decisions at intersections that actively consider the needs of pedestrians.

\subsection{Research Goals and Objectives}

The primary objective of this research is to incorporate pedestrian considerations into signal timing design at intersections and develop control tactics that can benefit pedestrians. Towards that end, this research seeks to answer three key questions:

1. What external, demographic, and behavioral factors influence crossing decisions and satisfaction with delay while crossing?

2. What are the impacts of control strategies to benefit pedestrians such as change in signal controller mode of operation, cycle length variation and permissive length 
variation on different modes at an intersection (light vehicle, heavy vehicle, bicycle and pedestrian)?

3. Operationally, what traffic regimes are best suited for these strategies?

\subsection{Overview of the Dissertation}

The remainder of this dissertation contains a compilation of three technical papers (Chapters 3, 4 and 5) that contribute to the overall research objectives. Thus, each chapter contains the relevant literature and a separate chapter reviewing the literature is not included. In Chapter Two, an overview of phasing, current pedestrian accommodation, and detection mechanisms at signalized intersections are presented. Chapter Three presents a comprehensive literature review pertaining to pedestrian crossing behavior at signalized intersections and describes the results of an intercept survey that was designed to understand pedestrian attitudes and perceptions regarding delay and crossing time and explore factors that influence crossing decisions. Chapter Four presents a summary of current literature on pedestrian delay estimation and measurement and describes the development of methods to automatically measure pedestrian actuations and delay in the signal controller. The results of the field deployment of two pedestrian friendly strategies and their impacts on pedestrian delay are described and discussed. Chapter Five evaluates the impact of change in signal controller mode of operation on delays experienced by all modes through the use of simulation models. Vehicular and pedestrian demands were varied and the resulting delays for all modes were compared between coordinated and free operation. A matrix that outlines the 
feasibility traffic regimes for each mode of operation is developed. Chapter Six summarizes the findings of this research and discusses policy implications along with some recommendations for future research. 


\section{BACKGROUND}

Intersections often have to accommodate a variety of modes and this is accomplished primarily through signal timing. This chapter reviews the current accepted standards for pedestrian service at signalized intersections. An overview of signal phasing, pedestrian timing schemes as well as a brief discussion of pedestrian detection methods at signalized intersections are presented in this chapter.

\subsection{Signal Timing}

The goal of signal timing at an intersection is to safely separate conflicting movements in time. Signal timing is important because it directly affects the quality of our transportation system (Koonce et al., 2008). Signal timing is often reflective of a region's transportation policy and goals and these goals determine the priority by which users are served at an intersection. Intersections in urban areas are places where a wide variety of travel modes - bicycles, pedestrians, autos, trucks and transit come together to compete for limited time and space. In the United States, traditional signal timing objectives have focused on encouraging vehicle progression and minimizing vehicular delay and stops. Traffic engineers may feel that they have to accommodate "alternative" modes at the expense of vehicular performance. This vehicle centric focus often can lead to large and often unnecessary delays for pedestrians. Prior research states that pedestrian frustration and likelihood of non-compliance increases when their delays exceed 30s (Dunn 1984, Van Houten 2006). As cities and jurisdictions around the country seek to actively 
promote walking as a transportation mode, providing pedestrians with the ability to cross safely and efficiently is critical.

\subsection{Phasing}

Phasing is the way in which various users are accommodated at signalized intersections (Koonce et al., 2008). Both vehicular and pedestrian phases exist at intersections serving vehicular and pedestrian movements respectively. A typical eight vehicle phase intersection with four pedestrian phases (labeled as P) is shown in Figure 2-1. The vehicle movements are depicted by solid lines and the pedestrian movements are shown as dashed lines.

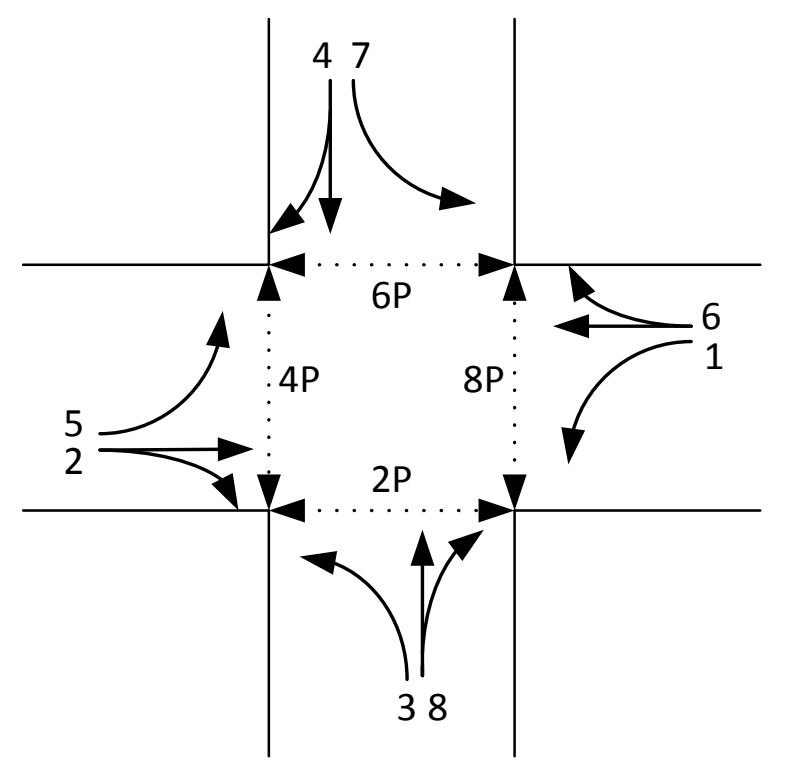

Figure 2-1 Vehicle and Pedestrian Phases at an Intersection

In the United States, signal phasing follows National Electrical Manufacturers Association (NEMA) standards. The NEMA ring and barrier diagram for a typical eight phase intersection in shown in Figure 2-2. This diagram typically consists of two rings 
and two barriers for one cycle. The rings allow non-conflicting movements to time concurrently whereas the barriers separate conflicting movements and prevent them from timing simultaneously. Pedestrians are accommodated through a few signal timing schemes that are discussed next.

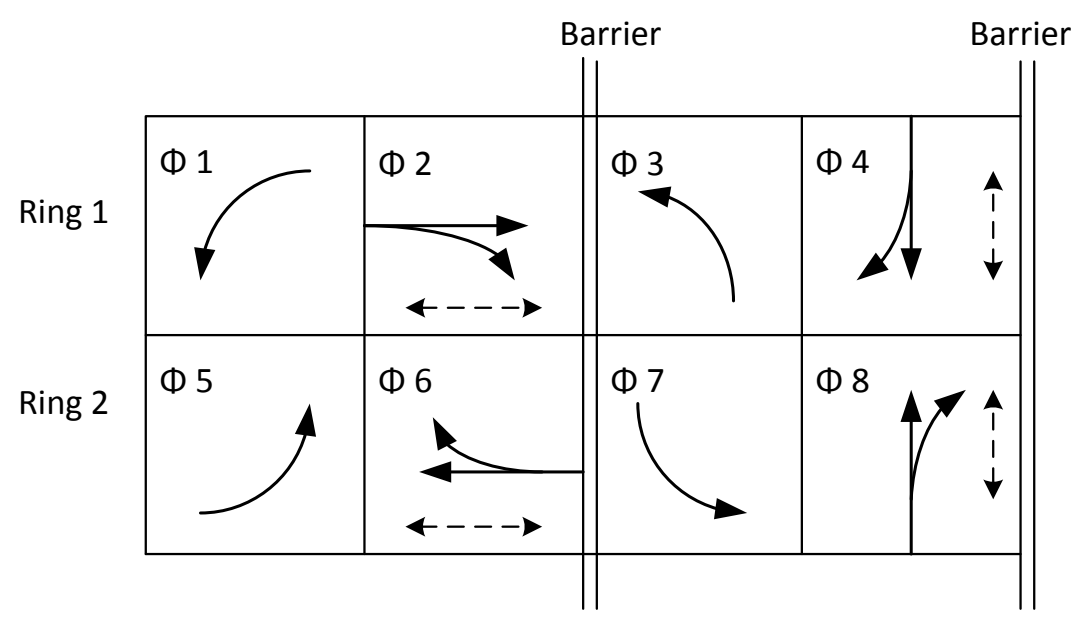

Figure 2-2 NEMA Ring and Barrier Diagram

\subsection{Pedestrian Signal Timing Schemes}

A pedestrian indication typically consists of three intervals: Walk, Flashing Don't Walk (FDW) and Solid Don't Walk. The intervals are communicated using the displays on the pedestrian signal head shown in Figure 2-3. During the Walk interval denoted by a steady walking person, pedestrians are permitted to step off the curb and start crossing the roadway. In the Flashing Don't Walk interval denoted by a flashing upraised hand, pedestrians are not supposed to start crossing, but those that have started crossing during the Walk phase may finish crossing. During the Solid Don't Walk interval denoted by the solid upraised hand, pedestrians are not permitted to enter the roadway. According the Manual of Uniform Traffic Control Devices (MUTCD), the minimum walk duration can 
be as low as $4 \mathrm{sec}$ and typically ranges between $4-7 \mathrm{sec}$ (MUTCD, 2009). Pedestrian clearance time (Flashing Don't Walk) is calculated as a ratio of crossing distance (length of crosswalk) to walking speed. Walking speeds vary among pedestrians; faster speeds are generally attributed to younger pedestrians and slower speeds are associated with older pedestrians. The MUTCD recommends a value of $3.5 \mathrm{ft} / \mathrm{s}$ to accommodate slower pedestrians.

Pedestrians at signalized intersections are accommodated through phasing schemes such as no pedestrian timing, concurrent timing, leading pedestrian interval, and pedestrian scramble or exclusive pedestrian phase.

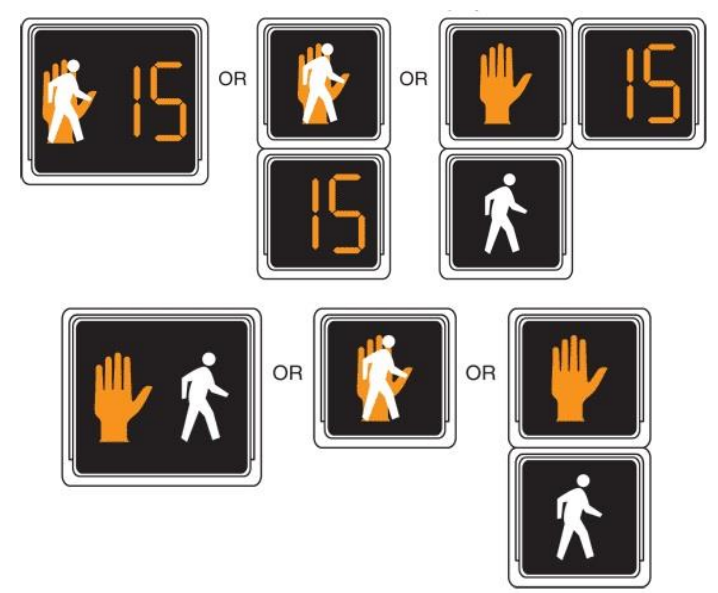

Figure 2-3 Pedestrian Signal Displays with and without Countdown Timers (Source: FHWA, Manual of Uniform Traffic Control Devices)

\subsection{No Pedestrian Timing}

In this scenario, no pedestrian timing parameters are input into the controller and no pedestrian signals are provided. Pedestrians may cross along with the parallel vehicle green indication. While the elimination of pedestrian timing may result in lower delays for vehicles, it may result in increased pedestrian vulnerability to conflicts especially if 
they have not finished crossing prior to the opposing vehicle green indication. The MUTCD recommends that engineering judgment be used to determine if pedestrian signal displays should be provided (MUTCD, 2009).

\subsection{Concurrent Pedestrian Signal Timing}

This is the most common way by which pedestrians are accommodated at signalized intersections. Pedestrian signal heads are provided for each crossing leg. In this type of phasing, the walk and flashing don't walk time concurrently with the parallel vehicle green indication. Turning vehicles (right and permissive left) are expected to yield to pedestrians in crosswalks. After the pedestrian clearance interval, the vehicle clearance follows and a solid don't walk is displayed for the pedestrians. The ring and barrier diagram for concurrent pedestrian signal timing is shown in Figure 2-4. The pedestrian phases $2 \mathrm{P}, 4 \mathrm{P}, 6 \mathrm{P}$ and $8 \mathrm{P}$ time concurrently with the vehicle phases $2,4,6$ and 8 respectively.

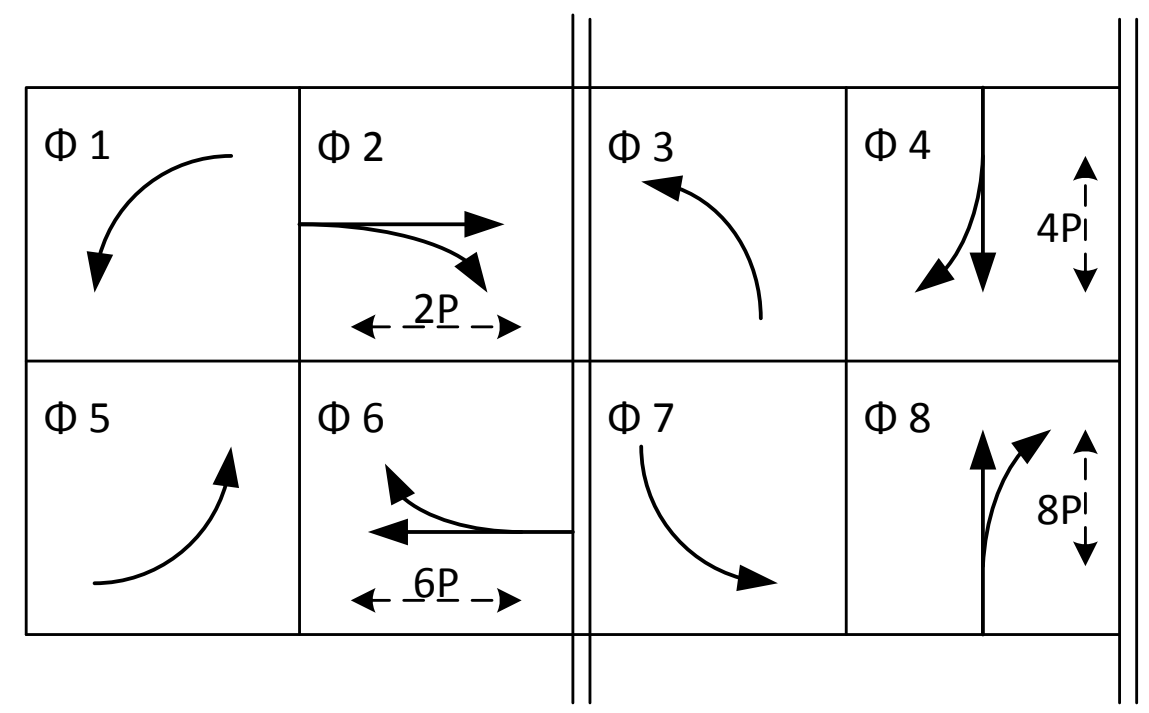

Figure 2-4 Concurrent Pedestrian Timing 


\subsection{Leading Pedestrian Interval}

In this type of timing, pedestrians are provided with an exclusive walk signal for a few sec, prior to the parallel vehicular green indication. This scheme provides pedestrians greater visibility and allows them to begin crossing before the vehicles can start their turning maneuvers. After the first few sec, the vehicular green indication for the parallel movement is served and the operation is similar to the concurrent timing as described above.

Figure 2-5 shows the ring and barrier diagram for LPI for phases 2 and 6 . The advantages of LPI are primarily safety related; studies have reported reduced conflicts between pedestrians and turning vehicles (Van Houten 2000, FHWA, Turner 2000). One study also reported a reduction in pedestrians yielding the right of way to turning vehicles (Fayish 2010). In addition to actual safety improvements, LPI's may also improve perceptions of safety. However, due to the lost time for vehicles during LPI, vehicular delays may increase.

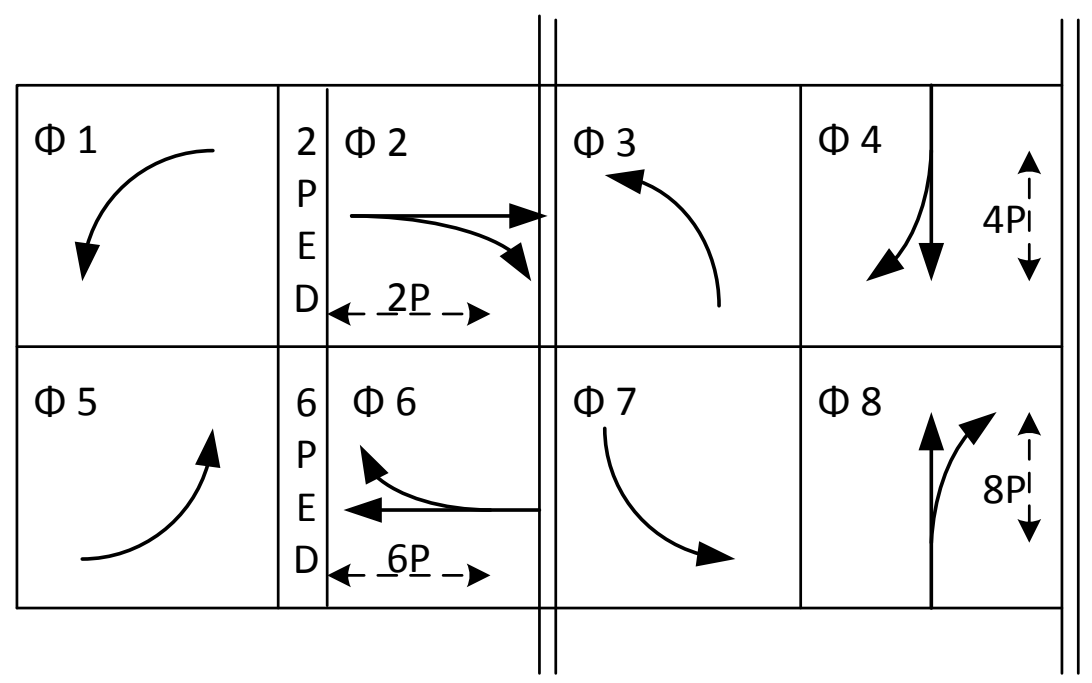

Figure 2-5 Leading Pedestrian Interval 


\subsubsection{Exclusive Pedestrian Phase}

An exclusive pedestrian phase (EPP) also known as a Barnes Dance or a Pedestrian Scramble is a type of phasing in which pedestrians are permitted exclusive use of the intersection including lateral and diagonal crossings while all vehicular traffic is stopped. The Walk signal for all crosswalks is displayed simultaneously. Figure 2-6 shows the ring diagram for EPP. While this phasing eliminates conflicts for pedestrians during EPP, it increases both vehicular and pedestrian delays due to increased cycle length. A few studies have documented increased pedestrian non-compliance due to the increased delays as a result of EPP implementation (Abrams 1977, Zeeger 1985, Zaidel 1987, Garder 1989, Bechtel 2004, Kattan 2009).

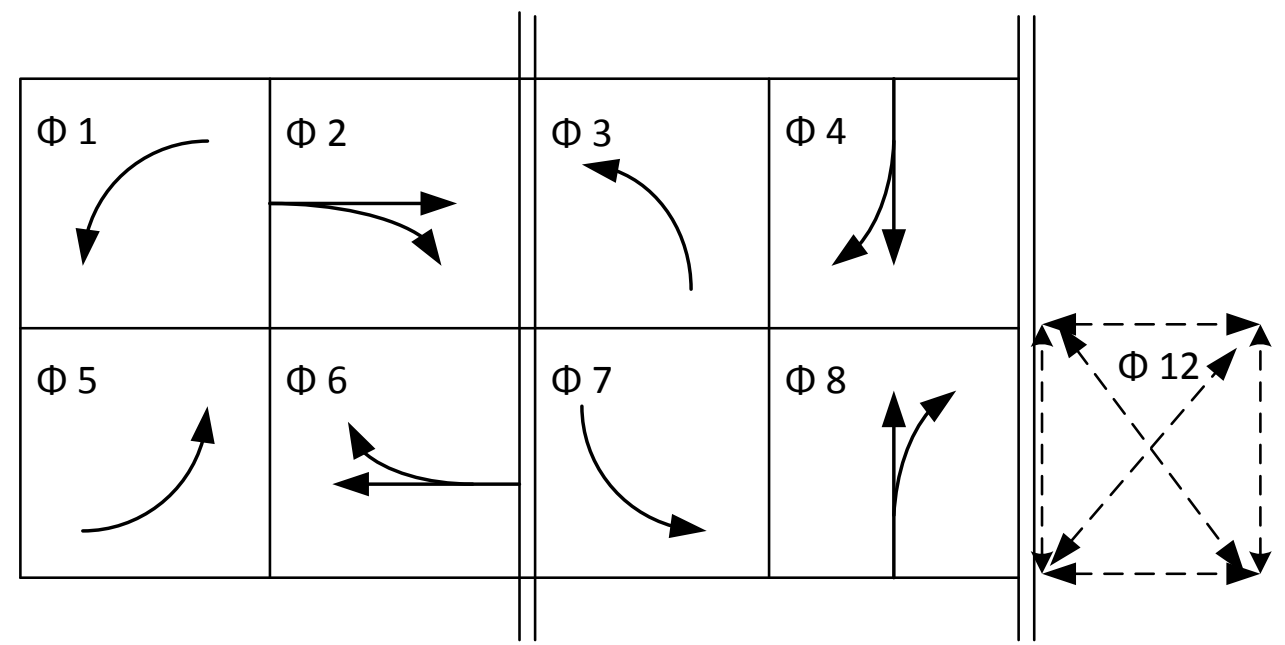

Figure 2-6 Exclusive Pedestrian Phase

\subsection{Pedestrian Detection at Intersections}

The primary purpose of detection at an intersection is to provide information to the signal controller that a user desires service (Koonce et al., 2008). The controller uses the information from multiple detectors to determine the sequence and duration of phases to 
be served. Detection technology for pedestrians is still evolving. The primary detection mechanism for pedestrians has been the use of pedestrian push buttons as shown in Figure 2-7. Detection through push buttons requires the pedestrians to press the button when they want to cross the street. The push button actuation is transmitted to the signal controller and a Walk display is provided to the pedestrians to safely cross the street.

Prior research has shown that not all individuals who want to cross will press the push button. Zeeger et al. found that only half of all pedestrians use the push button (Zeeger et al., 1985). The implication is that many pedestrians may cross against the signal in violation. Therefore, automated detection technologies have been suggested as an alternative way to detect pedestrians. Infrared, microwave and image processing technologies have been studied and show promise (FHWA, 2001). In addition to detection, infrared technologies are also being used to track a pedestrian's movement and extend crossing time if necessary. 


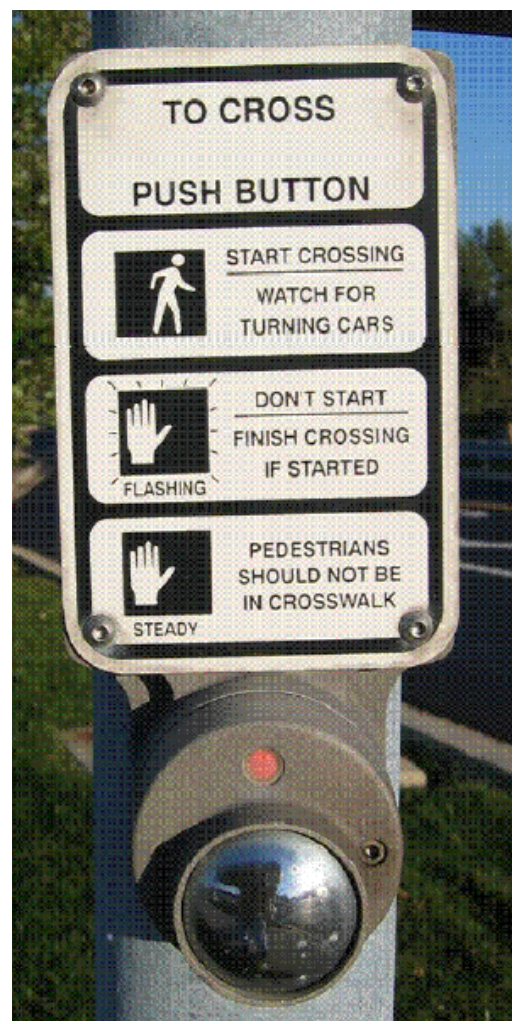

Figure 2-7 Pedestrian Push Buttons

(Source: Signal Timing Manual, 2008)

Another approach to serving pedestrians at intersections is to provide them with a Walk signal automatically every cycle. This approach termed as "Pedestrian Recall" eliminates the need for push buttons or other detection. It is typically used at locations with high pedestrian activity. The advantage with pedestrian recall is that pedestrians are automatically served each cycle irrespective of demand. The disadvantage is that it can result in an efficiency loss during certain time periods of the day when there are no pedestrians; however the signal controller has to still serve the walk and the clearance times leading to unnecessary delays. 


\subsection{Summary}

In this chapter, pedestrian signal timing schemes were reviewed. While concurrent phasing is the most common approach to accommodating pedestrians at intersections, other approaches such as leading pedestrian interval and Barnes Dance have been used selectively at intersections. While the benefits for both approaches are primarily safety related, intersection efficiency is reduced due to increased delay. While detection technology for pedestrians has been largely limited to pushbuttons, automated technologies are evolving.

It is imperative to understand pedestrian crossing behavior in order to design safe crossings. The next chapter reviews past literature on pedestrian crossing behavior and presents the findings of a survey that was designed to capture user perceptions and attitudes regarding delay, crossing time and crossing behavior decisions. 


\section{PEDESTRIAN BEHAVIOR AT INTERSECTIONS}

This chapter explores pedestrian attitudes and perceptions regarding safety, delay and crossing decisions at signalized intersections with the aim of gaining a better understanding of pedestrian behavior. The objective was to explore perceptions of delay and service as a function of characteristics that can be associated with the surrounding built environment or trip making behavior and some signal timing parameters. The chapter presents the findings of an intercept survey and through the use of regression models outlines factors that contribute to satisfaction with delay and motivations for crossing behavior. The chapter concludes with recommendations on measures to promote compliance and safe crossing behaviors at signalized intersections.

\subsection{Introduction}

Urban intersections are highly complex spaces, where the needs of all multimodal users have to be managed in the shared space, while simultaneously preventing conflicts. Past work has shown that pedestrian behavior is influenced by operational decisions at the intersection (Yanfeng et al., 2010). Traditionally traffic signal timing policies have often focused on minimizing delays for vehicular movements and pedestrian needs with respect to lower delays are not actively incorporated at intersections. Prioritizing vehicular movements may result in large delays for pedestrians, which in turn, can lead to pedestrian signal non-compliance. However, the motivations of pedestrian behavior with respect to their attitudes and perceptions of crossing conditions at intersections have not been adequately investigated. 
This chapter presents an effort to assess pedestrian attitudes and perceptions regarding safety, compliance and delay at signalized intersections via an intercept survey. It also explores differences in perceptions of satisfaction with delay based on type of pedestrian detection at intersections (recall vs. actuated), which has not been studied before.

\subsection{Background and Literature Review}

Pedestrian safety continues to be challenging in the United States and is one of the road user groups that is experiencing an increase in fatalities over the last few years (NHTSA, 2011). According to the National Highway Traffic Safety Administration (NHTSA) 4,743 pedestrians were killed and approximately 76,000 were injured in traffic crashes in the United States in 2012 (NHTSA, 2012). Figure 3-1 shows the trends in pedestrian fatalities from 2003 to 2012 .

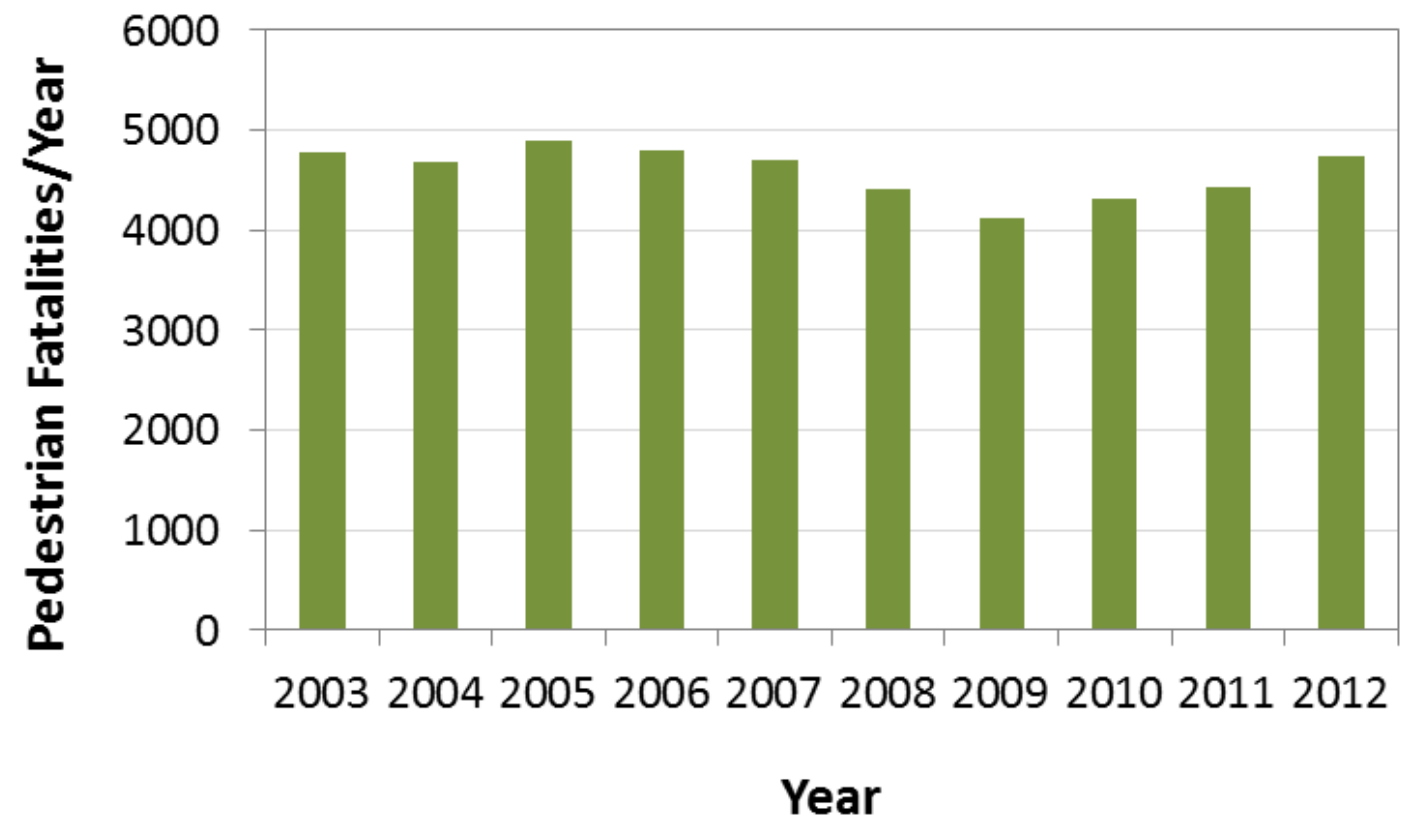

Figure 3-1 Pedestrian Fatality Trends (2003-2012) 
Out of these fatalities, $73 \%$ occurred in an urban setting. Older and younger populations are most at risk; older pedestrians $(65+)$ accounted for $20 \%$ of pedestrian fatalities and $9 \%$ of injuries and children younger than 15 accounted for $6 \%$ of pedestrian fatalities and $18 \%$ of pedestrian injuries (NHTSA, 2012). These specific age groups often rely on walking and modes other than driving to reach their destinations and therefore providing a safe walking environment is critical. Although the majority of these fatalities $(80 \%)$ did not occur at intersections, about $20 \%$ occurred at intersections.

Pedestrian crossing behavior and decisions are highly complex and varied. It is critical to understand crossing behavior in order to design safe intersections and crossings (Zaki, 2012). A safe pedestrian environment is critical to the development of a sustainable transportation system.

\subsubsection{Signal Violations and Compliance}

Pedestrian signals consist of three phases - walk indication, flashing don't walk and solid don't walk as described previously in section 2.3. Pedestrian signal violations occur when pedestrians disregard signal indications and rules. Signal compliance is a critical component of pedestrian crossing behavior. Some studies have established a link between non-compliance and traffic conflicts, leading to increased exposure (Struckman-Johnson 1989, Ayuso 2010). Shinar reported on a study of pedestrian crashes conducted in 13 U.S. cities, the findings indicated that a greater proportion of crashes could be attributed to risky and non-compliant behavior by pedestrians (Shinar 2008). Zeeger et al. analyzed pedestrian crashes in 15 cities and found a correlation between pedestrian crashes and 
signal violations; $42.7 \%$ of pedestrians were struck while violating the signal indication (Zeeger, 1985).

\subsubsection{Infrastructure}

Compliance was also influenced by type of pedestrian infrastructure at signalized intersections. Van Houten et al. found that providing confirmation of pushbutton activation through visual or auditory was beneficial in promoting pedestrian signal compliance (Van Houten et al. 2006). Bradbury et al. studied pedestrian compliance at six intersections in Seattle, Washington. They found that pedestrians were more likely to comply and wait for the walk indication if they pushed the button (Bradbury, 2012). They also recommend providing feedback to the pedestrian that their call for service has been registered by the signal controller. Several studies have evaluated the impact of pedestrian countdown timers on compliance and conflicts (Keegan et. al, 2003, Eccles et al. 2004). Keegan et al. evaluated before and after impacts of pedestrian countdown timers in Dublin, Ireland and found an increase in compliance after installation (Keegan et al. 2003). Eccles et. al. found a significant decrease in pedestrian vehicle conflicts post countdown timer installation (Eccles et al. 2004). This study showed that although pedestrians entered the intersection later in the pedestrian phase, they were able to clear the intersection before the green phase for the conflicting vehicle phase (Eccles et al. 2004). Hughes et al. conducted an evaluation of automated pedestrian detectors in conjunction with standard push buttons at signalized intersections (Hughes et al. 2000). 
Their findings revealed a decrease in non-compliance and pedestrian vehicle conflicts with automated pedestrian detection (Hughes et al. 2000).

\subsubsection{Crossing Characteristics}

Many crossing characteristics affect pedestrian crossing behavior. Yanfeng et al. found that wait time and pedestrian red phase length were important variables that dictated pedestrian behavior and could be key factors in improving intersection characteristics (Yanfeng et al. 2010). Many studies have shown that waiting time is a key factor in pedestrian non-compliance (Dunn et al. 1984, HCM 2010, Wang et al. 2011, Vallyon et al. 2011). The Highway Capacity Manual (HCM) states that when waiting times exceed 30s, they can induce frustration and increased likelihood of risk taking (HCM 2010). Wang et al. found that increase in wait time causes an increase in aggressive behavior and the likelihood of violation (Wang et al. 2011). A survey of pedestrians conducted in three cities in New Zealand found that perceived waiting times were longer than actual waiting times (Vallyon and Turner 2011). The study also found that after 20-30 sec of waiting, pedestrians level of frustration grew disproportionately compared to the actual waiting time (Vallyon and Turner 2011). Knoblauch et al. found that pedestrian walking speeds vary between compliers and non-compliers; complier speeds were slower than noncompliers (Knoblauch et al. 1996). Faster speeds were also observed with younger males, at locations with longer signal cycles and shorter pedestrian signal times (Knoblauch et al. 1996). Traffic volumes were also found to impact crossing behavior, with higher traffic volumes acting as a deterrent against risky behaviors (Hamed 2001). 


\subsubsection{Demographic Characteristics}

Age, gender and group settings also have an impact on pedestrian crossing characteristics. Crossing behavior of children was also found to be different compared to adults (Macgregor et al. 1999). Macgregor et al. found that $48 \%$ of unaccompanied children performed no visual search prior to crossing at signalized intersections (Macgregor et al. 1999). Diaz conducted a survey of pedestrian behavior in Santiago, Chile and found that younger people have more positive perception towards violations and report more violations than older adults (Diaz 2002). Wang et al. found that older pedestrians could endure longer waiting times than younger pedestrians (Wang et al., 2011). A few studies found that males were associated with higher levels of noncompliance compared to females (Diaz 2002, Wang et al. 2011). Presence of a group was found to impact crossing behavior, with pedestrians following the crossing actions of others at intersections with regard to compliance as well as non-compliance (Yanfeng et al. 2010, Bradbury et al. 2012, and Wang et al. 2011). Trip purpose also affects crossing behavior; Wang et al. found that pedestrians undertaking either work or school trips waited for shorter time (Wang et al. 2011).

While past research has explored some factors that influence crossing behavior and decisions, no previous study has explored pedestrian perceptions of delay based on type of signal phasing based on infrastructure (actuated or recall). This chapter attempts to fill that gap. 


\subsection{Methodology}

To gather information about the attitudes and perceptions that determine crossing decisions and behavior, an intercept survey was administered to pedestrians at four signalized intersections in Portland, OR. A pilot instrument was administered at an intersection on the campus of Portland State University prior to the actual survey. The

pilot tested the instrument (computer tablet vs. paper), wording of questions, and recruiting strategies for the intercept and served to train the survey staff. The experience from a pilot survey revealed that tablet instrument was preferred due to ease of use, faster administration, and generally more favorable perception of the survey. The tablets were found to be challenging to use in rain or bright sun (due to glare) and somewhat harder for older adults to use. Survey respondents were given a choice to either self-record their responses or indicate their responses to the surveyors, who recorded it for them. The pilot survey also revealed that it was harder to recruit and survey people walking in groups, pedestrians carrying on conversations over a mobile phone and pedestrians walking with earphones, listening to music. Therefore special emphasis was placed on the surveyors to actively recruit these populations during survey administration. The piloted survey instrument included questions that were designed to assess respondents' perception of crossing time, delay in minutes, trip length in miles and their understanding of pedestrian signals. However, prior work has shown that in general, people are poor at estimating time (Vallyon and Turner, 2011). Hence, the questions were revised to instead gather respondents' satisfaction with crossing and waiting times, information about trip 
duration, frequency and purpose, their perceptions of safety and determinants of crossing decisions.

Table 3-1 shows the survey instrument. The final survey was designed to be short (11 questions, less than 3 minutes to complete) in order to reduce the burden on the respondent and increase response rates. To that end, the survey was designed such that the surveyor entered certain demographic (gender) and other information (whether the respondent was in a group, presence of kids, surveyed location and crosswalk) based on observation, after the respondents completed the survey. 
1. Are you walking?

a. To public transportation b. From public transportation c. Neither

2. What is the purpose of your trip?

a. Work b. School/college c. Home d. Accompanying minor e. Shopping f. Eating out g. Visiting friends/rec activities h. Exercise i. Other

3. How long is your walking trip?

a. $<5$ mins b. $5-10$ mins c. $10-15$ mins d. $>15$ mins

4. How often do you walk through this intersection?

a. First time b. Less than one day a month c. 1-3 days/month d. 1-3 days/week e. 4 or more days a week

5. How satisfied are you with the amount of time you have to wait before crossing at this intersection?

a. Very satisfied b. Somewhat satisfied c. Somewhat dissatisfied d. Very dissatisfied

6. How satisfied are you with the amount of time the signal gives you to cross at this intersection?

a. Very satisfied b. Somewhat satisfied c. Somewhat dissatisfied d. Very dissatisfied

7. In general how safe do you feel crossing this intersection?

a. Very safe b. Somewhat safe c. Somewhat unsafe d. Very unsafe

8. Please indicate your level of agreement with the following statement: My crossing decisions are influenced by concerns about safety.

a. Strongly agree b. agree c. Disagree d. Strongly disagree

9. Please indicate your level of agreement with the following statement: My crossing decisions are influenced by concerns about whether I am violating traffic code (jaywalking, crossing against the signal etc.).

a. Strongly agree $b$. agree c. Disagree d. Strongly disagree

10. What is your age?

a. $18-25$ b. $26-39$ c. $40-65$ d. $66-75$ e. $76^{+}$

11. What is the zip code of your home address?

Four locations in Portland, OR were chosen for survey administration: NE Multnomah

Street and NE $9^{\text {th }}$ Avenue, NE Multnomah Street and NE $13^{\text {th }}$ Avenue, NE Sandy

Boulevard and NE $39^{\text {th }}$ Avenue and NE Sandy Boulevard and NE $43^{\text {rd }}$ Avenue. The

number of intersections chosen was governed by availability of resources (survey staff, time and equipment). Figure 3-2 shows the aerial views of the four intersections. The 
land use surrounding the intersections on Multnomah Boulevard was commercial mixed with retail. A large shopping mall was adjacent to both surveyed intersections on this corridor. The intersection at Sandy and $39^{\text {th }}$ was close to a major freeway (I-84) and the land use was mixed retail and service. At Sandy and $43^{\text {rd }}$, there was a large grocery store that was the primary destination for many pedestrians crossing that intersection. The intersection of Sandy at $39^{\text {th }}$ had only one crosswalk for pedestrians to cross Sandy, compared to two crosswalks crossing the major street at all other surveyed intersections.

Due to the prevailing land uses at these locations, shopping and work are primary destinations for many trips. These locations were selected based on a set of criteria which included: moderate levels of pedestrian activity ( $>300$ actuations per day), variability with pedestrian signal control (actuated vs. recall intersections) and presence of Type 2070 signal controllers which accorded the ability to record pedestrian activity and delay. Actuated intersections (Multnomah $/ 13^{\text {th }}$, Sandy $/ 39^{\text {th }}$ ) were equipped with pushbuttons for pedestrians to activate in order to cross the street. The pedestrian phases at recall intersections (Multnomah $/ 9^{\text {th }}$, Sandy $/ 43^{\text {rd }}$ ) are automatically served every cycle irrespective of demand. 


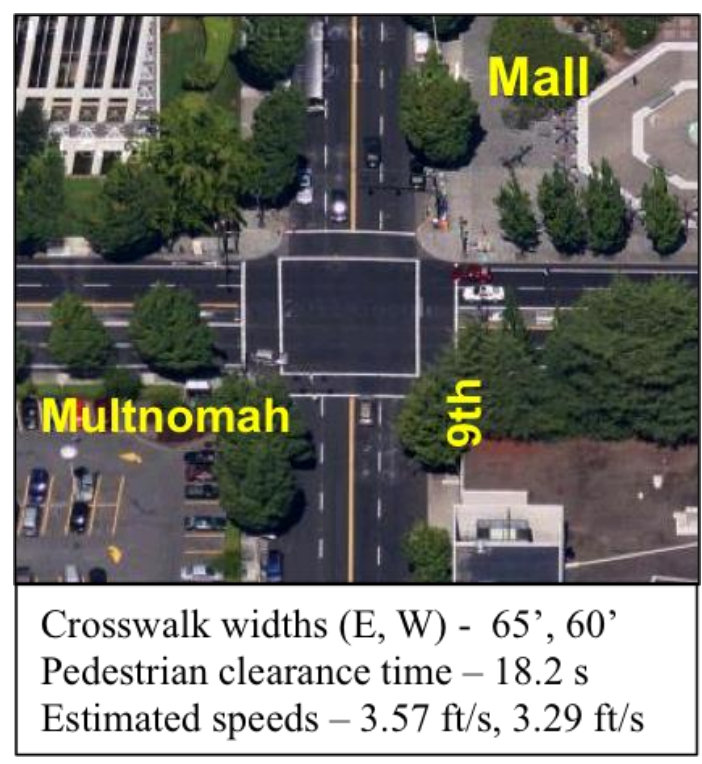

a) Multnomah and $9^{\text {th }}$

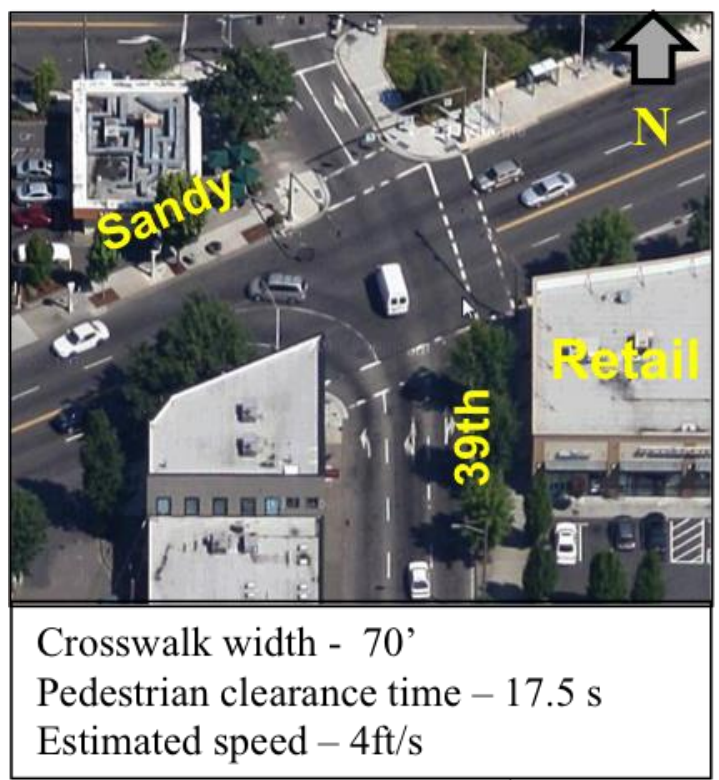

c) Sandy and $39^{\text {th }}$

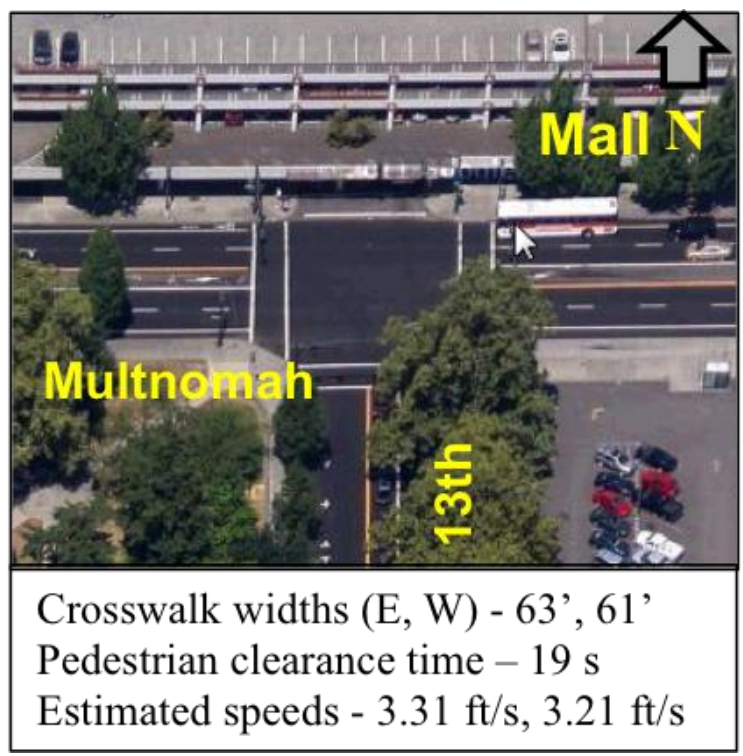

b) Multnomah and 13th

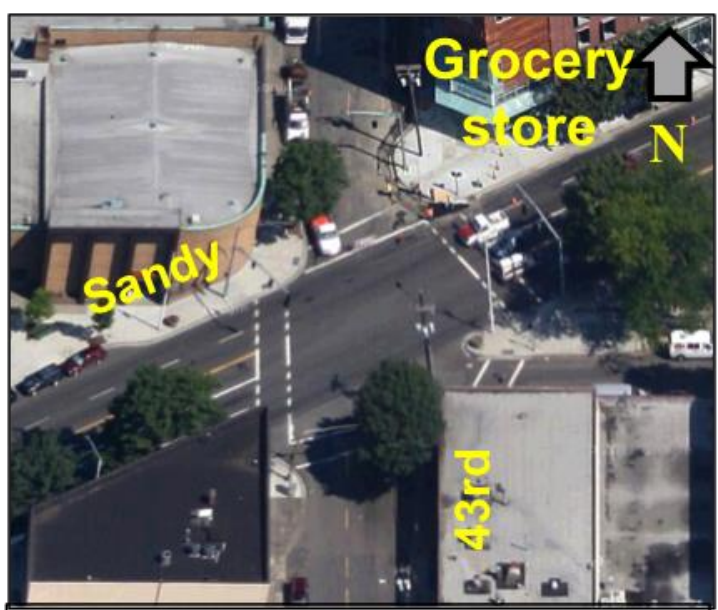

Crosswalk widths (E, W) - 72', 56'

Pedestrian clearance time - $19 \mathrm{~s}$

Estimated speeds $-3.78 \mathrm{ft} / \mathrm{s}, 2.94 \mathrm{ft} / \mathrm{s}$

d) Sandy and 43rd

\section{Figure 3-2 Survey Locations}

Data collection commenced on May 8 and was completed on May 31,2013. Three time periods were chosen for survey administration, two of them coinciding with traditional AM and PM vehicle peaks (7-9 AM, 4-6 PM) and the third corresponding to vehicle off- 
peak (11 AM - 1 PM) but characterized by higher pedestrian volumes. The length of each time period ( 2 hours) was chosen to align with the typical length of peak period. At each location, a minimum of three surveyors were present during the assigned days and time periods; two surveyors to administer the survey and the third surveyor to record the number of crossing pedestrians in crosswalks. The survey was administered only to those pedestrians who crossed the major street, as these pedestrian phases were actuated at two intersections. Minor street pedestrian phases were always on recall at the four locations.

\subsection{Descriptive Statistics}

A total of 529 respondents were solicited to participate in the survey; 367 respondents agreed to take the survey and 162 declined. No incentive was offered to the respondents for participation in the survey. More males than females (58\% vs. $42 \%)$ declined to participate in the survey. Also, $19 \%$ of the non-respondents were walking in a group and $3 \%$ of them had children accompanying them on their trip. Table 3-2 provides an overview of the survey sample for all sampled days and time periods. Prior to the survey commencement, a sampling rate of $10 \%$ was targeted for each time period. The response rate varied from $66 \%-77 \%$, while the sampling rate varied from $16 \%-33 \%$, thus meeting our set targets of $10 \%$ of the total crossing pedestrian volume for the time period at each intersection.

Table 3-2 Summary Survey Response Statistics by Intersection

\begin{tabular}{|c|c|c|c|c|c|}
\hline Location & Completed & Refused & Crossing Peds & Response Rate (\%) & \% Sampled \\
\hline Multnomah \& $9^{\text {th }}$ & 140 & 60 & 1242 & 70 & 16 \\
\hline Multnomah $\& 13^{\text {th }}$ & 81 & 42 & 757 & 66 & 16 \\
\hline Sandy $\& 39^{\text {th }}$ & 53 & 16 & 210 & 77 & 33 \\
\hline Sandy $\& 43^{\text {rd }}$ & 93 & 44 & 488 & 68 & 28 \\
\hline Total & 367 & 162 & 2697 & & \\
\hline
\end{tabular}


Table 3-3 shows the demographic characteristics of the surveyed sample by intersection. Overall, $49 \%$ of the respondents were between $40-65$ years of age and the gender split was fairly even among the respondents. As expected, the majority of the respondents $(83 \%)$ were residents of the Portland metropolitan area. Approximately $97 \%$ of the respondents surveyed did not have children along with them on their trip and $87 \%$ were not in a group. Higher proportions of younger adults and more individuals in groups were captured at the Multnomah locations compared to the Sandy locations. More females than males were captured at the Sandy locations. These differences in pedestrian characteristics can be perhaps attributed to differences in land use surrounding the intersections.

Table 3-4 summarizes the trip characteristics that were gathered from the participants. Overall, $37 \%$ of the participants were walking either to or from public transportation. The majority of the walk trips undertaken by the respondents $(72 \%)$ were less than 10 minutes in length and $47 \%$ of the respondents surveyed used the intersections on a very frequent basis. Of the surveyed respondents, the most common trip purpose was shopping (31\%), followed by work (29\%) and eating out (16\%). More respondents were captured while on work-based trips at Multnomah $/ 9^{\text {th }}$; similarly shopping was the predominant trip purpose at Multnomah/ $13^{\text {th }}$ and Sandy $/ 43^{\text {rd }}$. As expected these trip purposes were aligned with the surrounding land use and destinations at these intersections. 
Table 3-3 Descriptive Statistics

\begin{tabular}{l|rr|rr|r}
\hline \multicolumn{1}{c|}{ Sample Overview } & \multicolumn{2}{|c|}{$\%$} & \multicolumn{2}{c}{$\%$} & \multicolumn{1}{c}{$\%$} \\
\hline & \multicolumn{2}{|c|}{ Multnomah } & \multicolumn{2}{c}{ Sandy } & Total \\
& $\mathbf{9}^{\text {th }}$ & $\mathbf{1 3}^{\text {th }}$ & $\mathbf{3 9}^{\text {th }}$ & $\mathbf{4 3}^{\text {rd }}$ & \\
\hline Age & & & & & \\
$18-25$ & 5.0 & 15.2 & 13.2 & 7.5 & 9.1 \\
$26-39$ & 41.0 & 36.7 & 26.4 & 29.0 & 34.9 \\
$40-65$ & 50.4 & 41.8 & 50.9 & 50.5 & 48.6 \\
$66-75$ & 3.6 & 6.3 & 3.8 & 11.8 & 6.3 \\
$76+$ & 0.0 & 0.0 & 5.7 & 1.1 & 1.1 \\
Gender & & & & & \\
Male & 53.6 & 55.6 & 45.3 & 48.4 & 51.5 \\
Female & 46.4 & 44.4 & 54.7 & 51.6 & 48.5 \\
Resident status & & & & & \\
Non-local & 17.1 & 23.5 & 18.9 & 11.8 & 17.4 \\
Local & 82.9 & 76.5 & 81.1 & 88.2 & 82.6 \\
Presence of children & & & & & \\
Yes & 0.7 & 4.9 & 3.8 & 2.2 & 2.5 \\
No & 99.3 & 95.1 & 96.2 & 97.8 & 97.5 \\
Groups & & & & & \\
Yes & 16.4 & 14.8 & 13.2 & 7.5 & 13.4 \\
No & 83.6 & 85.2 & 86.8 & 92.5 & 86.6 \\
N & $\mathbf{1 4 0}$ & $\mathbf{8 1}$ & $\mathbf{5 3}$ & $\mathbf{9 3}$ & $\mathbf{3 6 7}$ \\
\hline
\end{tabular}


Table 3-4 Trip Characteristics by Intersection

\begin{tabular}{|c|c|c|c|c|c|}
\hline \multirow[t]{3}{*}{ Sample Overview } & \multicolumn{2}{|c|}{$\%$} & \multicolumn{2}{|c|}{$\%$} & $\%$ \\
\hline & \multicolumn{2}{|c|}{ Multnomah } & \multicolumn{2}{|c|}{ Sandy } & \multirow[t]{2}{*}{ Total } \\
\hline & $9^{\text {th }}$ & $13^{\text {th }}$ & $39^{\text {th }}$ & $43^{\text {rd }}$ & \\
\hline \multicolumn{6}{|l|}{ Public transit } \\
\hline To public transportation & 21.4 & 25.9 & 20.8 & 7.5 & 18.8 \\
\hline From public transportation & 13.6 & 30.9 & 18.9 & 14.0 & 18.3 \\
\hline Neither & 65.0 & 43.2 & 60.4 & 78.5 & 62.9 \\
\hline \multicolumn{6}{|l|}{ Trip length } \\
\hline$<5$ mins & 43.6 & 49.4 & 35.8 & 47.3 & 44.7 \\
\hline $5-10 \mathrm{mins}$ & 28.6 & 17.3 & 35.8 & 31.2 & 27.8 \\
\hline $10-15$ mins & 13.6 & 9.9 & 9.4 & 8.6 & 10.9 \\
\hline$>15$ mins & 14.3 & 23.5 & 18.9 & 12.9 & 16.6 \\
\hline \multicolumn{6}{|l|}{ Intersection usage } \\
\hline First time & 6.4 & 9.9 & 1.9 & 4.3 & 6.0 \\
\hline Less than one day per month & 9.3 & 16.0 & 5.7 & 2.2 & 8.4 \\
\hline $1-3$ days per month & 15.7 & 13.6 & 13.2 & 12.9 & 14.2 \\
\hline $1-3$ days per week & 18.6 & 28.4 & 35.8 & 21.5 & 24.0 \\
\hline 4 or more days per week & 50.0 & 32.1 & 43.4 & 59.1 & 47.4 \\
\hline \multicolumn{6}{|l|}{ Trip purpose } \\
\hline Work & 40.7 & 19.8 & 26.4 & 20.4 & 28.9 \\
\hline School/college & 1.4 & 4.9 & 0.0 & 0.0 & 1.6 \\
\hline Accompany minor & 0.0 & 1.2 & 0.0 & 0.0 & 0.3 \\
\hline Home & 14.3 & 9.9 & 18.9 & 16.1 & 14.4 \\
\hline Shopping, errands & 21.4 & 33.3 & 24.5 & 47.3 & 31.1 \\
\hline Eating out/coffee & 17.9 & 12.3 & 24.5 & 10.8 & 15.8 \\
\hline Visiting friends/recreation & 2.1 & 8.6 & 1.9 & 3.2 & 3.8 \\
\hline No particular destination/exercise & 2.1 & 7.4 & 1.9 & 2.2 & 3.3 \\
\hline Other & 0.0 & 2.5 & 1.9 & 0.0 & 0.8 \\
\hline $\mathbf{N}$ & 140 & 81 & 53 & 93 & 367 \\
\hline
\end{tabular}

Respondents were also asked to answer a series of questions ranging from perceptions of safety and satisfaction with delay and waiting time as well as their attitudes regarding crossing decisions. Table 3-5 shows the findings related to pedestrian perceptions and attitudes. Overall, a majority of the respondents $(80 \%)$ felt that the locations were either somewhat or very safe. Similarly, a majority of the respondents $(86 \%)$ were somewhat or very satisfied with delay and crossing times. Dissatisfaction with delay and crossing times were higher at actuated intersections compared to recall intersections. Also, $86 \%$ of the respondents also agreed with the statement "my crossing decisions are influenced by 
concerns about safety". Fewer respondents $(60 \%)$ agreed with the statement "my crossing decisions are influenced by concerns about whether I am violating traffic code" (jaywalking, crossing against the signal etc.). The findings clearly indicate that individuals value safety higher than compliance for crossing decisions.

Table 3-5 Attitudes and Perceptions

\begin{tabular}{|c|c|c|c|c|c|}
\hline \multirow{3}{*}{ Question } & \multirow{2}{*}{\multicolumn{2}{|c|}{$\frac{\%}{\text { Multnomah }}$}} & \multirow{2}{*}{\multicolumn{2}{|c|}{$\begin{array}{c}\% \\
\text { Sandy }\end{array}$}} & $\%$ \\
\hline & & & & & \multirow{2}{*}{ Total } \\
\hline & 9th & 13th & $39^{\text {th }}$ & 43rd & \\
\hline \multicolumn{6}{|c|}{ Satisfaction with crossing time } \\
\hline Very Dissatisfied & 0.7 & 4.9 & 3.8 & 1.1 & 2.2 \\
\hline Somewhat Dissatisfied & 8.7 & 11.1 & 15.4 & 11.8 & 11.0 \\
\hline Somewhat Satisfied & 38.4 & 58.0 & 30.8 & 29.0 & 39.3 \\
\hline Very Satisfied & 52.2 & 25.9 & 50.0 & 58.1 & 47.5 \\
\hline \multicolumn{6}{|l|}{ Satisfaction with Delay } \\
\hline Very Dissatisfied & 2.2 & 12.3 & 5.7 & 0.0 & 4.4 \\
\hline Somewhat Dissatisfied & 5.8 & 18.5 & 15.1 & 7.5 & 10.4 \\
\hline Somewhat Satisfied & 46.8 & 51.9 & 41.5 & 43.0 & 46.2 \\
\hline Very Satisfied & 45.3 & 17.3 & 37.7 & 49.5 & 39.1 \\
\hline \multicolumn{6}{|l|}{ Perception of safety } \\
\hline Very Unsafe & 2.9 & 4.9 & 5.7 & 7.5 & 4.9 \\
\hline Somewhat Unsafe & 15.8 & 11.1 & 15.1 & 18.3 & 15.3 \\
\hline Somewhat Safe & 33.8 & 56.8 & 32.1 & 43.0 & 41.0 \\
\hline Very Safe & 47.5 & 27.2 & 47.2 & 31.2 & 38.8 \\
\hline \multicolumn{6}{|c|}{ Crossing decision based on safety } \\
\hline Strongly Disagree & 2.9 & 1.2 & 5.7 & 1.1 & 2.5 \\
\hline Disagree & 12.4 & 17.3 & 11.3 & 6.5 & 11.8 \\
\hline Agree & 43.1 & 43.2 & 34.0 & 36.6 & 40.1 \\
\hline Strongly Agree & 41.6 & 38.3 & 49.1 & 55.9 & 45.6 \\
\hline \multicolumn{6}{|c|}{ Crossing decision based on compliance } \\
\hline Strongly Disagree & 14.5 & 11.1 & 13.2 & 9.7 & 12.3 \\
\hline Disagree & 26.8 & 29.6 & 20.8 & 32.3 & 27.9 \\
\hline Agree & 39.9 & 39.5 & 35.8 & 31.2 & 37.0 \\
\hline Strongly Agree & 18.8 & 19.8 & 30.2 & 26.9 & 22.7 \\
\hline $\mathbf{N}$ & 138 & 81 & 52 & 93 & 364 \\
\hline
\end{tabular}


A crosstab analysis was conducted in SPSS to determine whether these attitudes and perceptions varied by gender or age. Table 3-6 shows the statistically significant associations as indicated by a chi-square test. Significant differences in satisfaction associated with delay and crossing time by age were observed. More respondents in the 66-75 age group (21.7\%) expressed dissatisfaction with crossing time, followed by the 18-25 age group (21.2\%). The dissatisfaction with crossing time observed with the older age group may be related to insufficient crossing time (due to slower speeds), the dissatisfaction seen in the 18-25 age group is surprising. More respondents in age group $18-25(21.2 \%)$ reported dissatisfaction with the amount of time they had to wait before crossing at the intersections. Significant differences in perception of safety with respect to age were also observed, with younger respondents perceiving greater safety while crossing compared to older respondents. More women compared to men (55.4\% vs. $36.5 \%$ ) strongly agreed that their crossing decisions were based on safety considerations. Conversely more men than women (43.5\% vs. $36.9 \%$ ) disagreed (somewhat or strongly) that their crossing decisions were influenced by concerns about compliance.

Table 3-6 Chi Square Tests

\begin{tabular}{l|rr}
\hline \multicolumn{1}{c|}{ Variable 1 } & Variable 2 & $\boldsymbol{p}$-value \\
\hline \multirow{2}{*}{ Crossing time } & Gender & 0.500 \\
& Age & $\mathbf{0 . 0 0 2}$ \\
Delay & Gender & 0.301 \\
& Age & $\mathbf{0 . 0 1 3}$ \\
Safety & Gender & 0.377 \\
Crossing decisions - Safety & Age & $\mathbf{0 . 0 0 4}$ \\
Crossing decisions - Traffic code & Gender & $\mathbf{0 . 0 0 1}$ \\
& Age & $\mathbf{0 . 0 0 0}$ \\
& Gender & $\mathbf{0 . 0 2 8}$ \\
& Age & 0.318 \\
\hline
\end{tabular}

Bolded values are significant at $95 \%$ confidence 


\subsection{Model Development and Results}

To assess the factors that contribute to satisfaction with delay and motivations for crossing behavior, regression models were estimated. The survey measured four levels of satisfaction with delay and safety and four levels of agreement with crossing behavior choices as shown in Table 3-5. We initially explored the use of multinomial logit models to explore respondents' satisfaction with delay. However, in order to explore differences between locations, the small sample required the variables be recoded into two categories (for questions 5-9 in Table 3-1). For example, in the case of the crossing decisions variables (questions 8 and 9 in Table 3-1) strongly agree and agree responses were combined into one category, strongly disagree and disagree responses were combined into the other category. Thus, binary logistic regression models were developed using SPSS. These models are typically used to model relationship between categorical response variable and one or more explanatory variables that may be continuous or categorical. The dependent variable is not continuous, instead has only two possible outcomes. The advantage of logistic regression is the ability to interpret regression coefficients in terms of odds. The logistic regression equation is typically expressed as

$$
\ln \left(\frac{p_{i}}{1-p_{i}}\right)=\beta_{0}+\beta_{1} X_{1}
$$

where $p_{i}$ is the predicted probability and $X_{1}$ is the predictor variable. The left side of the equation is the logit function. The coefficients for predictors are often expressed as odds ratios. The odds ratio for each predictor is estimated as $\mathrm{e}^{\mathrm{B}}$ or $\exp (\mathrm{B})$ (Cohen et al., 2002). The odds ratio indicates the amount the odds of being in a group are multiplied when the 
predictor is incremented by one unit (Cohen et al., 2002). For each explanatory variable that was categorical, dummy variables were created and used to contrast the different categories. For all variables, the reference category for the dummy variable was chosen to be the category with the most number of observations. A correlation analysis was conducted between all the independent variables and Pearson's correlation coefficient was used to filter out the correlated variables. Crossing time was found to be significantly correlated with both delay and safety (Pearson coefficient $>0.4$ ); hence crossing time was not used in the models.

A total of six models were estimated to compare and contrast across intersections and perception questions. One model predicts the likelihood that crossing decisions were based on concerns about safety; another predicts likelihood that decisions were motivated by concerns about compliance. The dependent variable in these models is the probability that individuals agree that their crossing decisions are based on safety and compliance respectively. The data used in these two models were pooled from all surveyed intersections. The basis for model development was that crossing decisions were a function of demographics, trip characteristics and perceptions of delay and safety. Multiple iterations of the models were run and some non-significant variables were removed. The final model results for crossing decisions for safety $(-2 L L=242.53$, Model $\left.\chi^{2}=55.42\right)$ and compliance $\left(-2 \mathrm{LL}=457.82\right.$, Model $\left.\chi^{2}=31.17\right)$ are presented in Table 3-7. Since these models are exploratory in nature, some non-significant variables are retained in the model so as to understand their effect on the dependent variable, based on 
the sign of their coefficient. The reference categories for all the variables included in the models are listed in footnotes below each table.

Four sets of models were estimated to predict the likelihood of satisfaction with delay; one set each at actuated and recall intersections to reveal differences in location while controlling for type of detection (push buttons vs. automatic) and the other set was estimated at the corridor level (Multnomah vs. Sandy) to illustrate differences based on type of pedestrian control (actuated vs. recall). The data used in each of these models were a subset of the original data set. The dependent variable in these models is the probability that an individual is satisfied with delay at an intersection. Final models for actuated $\left(-2 \mathrm{LL}=126.15\right.$, Model $\left.\chi^{2}=24.46\right)$ and recall $\left(-2 \mathrm{LL}=99.86\right.$, Model $\left.\chi^{2}=26.57\right)$ intersections are shown in Table 3-8. Finally, Multnomah $\left(-2 \mathrm{LL}=155.08\right.$, Model $\chi^{2}=$ 33.33) and Sandy $\left(-2 L L=65.07\right.$, Model $\left.\chi^{2}=43.96\right)$ models are shown in Table 3-9.

Table 3-7 presents the models for crossing decisions. The significant predictors ( $p$ $<0.1)$ for the likelihood that crossing decisions were based on safety were compliance, presence in a group, work-based trip, home-based trip, recreational trip, trip duration greater than 15 minutes, older adults (age $>75)$ and perceptions of safety. Gender, use of public transportation, location, perception of delay and trip frequency were not significant factors. Respondents were more likely to agree that safety was a factor in their crossing decisions when they felt that compliance also played a role. Compared to shopping trips, respondents on home-based trips were associated with 4 times the odds of agreement with the statement that their crossing decisions were based on safety. Conversely work trips, presence of groups, longer trip lengths, older adults and 
respondents who perceived the intersections as safe were less likely to agree that safety was a factor in their crossing decisions. Respondents crossing in groups had $60 \%$ lower odds regarding concerns about safety while crossing compared to respondents crossing alone. Although not significant, compared to men, women were more likely to base their crossing decisions on safety concerns. Despite the poor model fit, a few observations can be made about pedestrian concerns about compliance. Respondents who undertook a recreational trip, whose trip length was between 10-15 minutes and, who identified safety as a determinant of crossing decision were more likely to agree that concerns with compliance were a factor while crossing. Although not significant, younger adults were less likely to make crossing decisions based on compliance. The poor model fit may be due to the small sample size or respondents' sensitivity about answering questions about compliance and/or lack of clarity about the intent of the question. Future work should explore question wording and respondent sensitivity in the survey instrument design. In behavior research, it is challenging to predict crossing behavior with explanatory variables that can fully capture pedestrian attitudes and perceptions (as seen with the low model pseudo $\mathrm{R}^{2}$ ). Factors that were not included in these models but could possibly influence crossing behavior include familiarity with intersections and attitudinal preferences regarding risk taking.

Table 3-8 shows the models for actuated and recall intersections. Initial runs of the models hypothesized that satisfaction with delay is a function of trip purpose, trip length, location, trip frequency, age, gender, presence of kids, group status, whether the respondent was local, safety perceptions and attitudes regarding crossing decisions. For 
the actuated intersections, safety perception and home-based trips were significant predictors of respondents' likelihood to be satisfied with delay. Compared to frequent users of the intersection ( 4 or more times per week), infrequent users ( $<1$ day per month) were associated with 4 times the odds of being satisfied with delay.

At intersections that were recalled (pedestrian phases were automatically served during each cycle), positive perceptions of safety resulted in satisfaction with delays. Respondents who perceived the intersection as either somewhat or very safe were associated with $622 \%$ higher odds with respect to delay satisfaction. Users of public transportation, pedestrians on a recreational trip and pedestrians whose trip lengths were short (5-10 minutes) had lower odds of being satisfied with the waiting time at these intersections. 
Table 3-7 Models of Influences on Crossing Decisions

\begin{tabular}{|c|c|c|c|c|c|c|c|}
\hline \multicolumn{4}{|c|}{ Crossing Decisions - Safety } & \multicolumn{4}{|c|}{ Crossing Decisions - Compliance } \\
\hline & B & S.E. & OR & & B & S.E. & OR \\
\hline $\begin{array}{l}\text { Intercept } \\
\text { Demographics }\end{array}$ & 2.948 & 0.862 & 19.074 & $\begin{array}{l}\text { Intercept } \\
\text { Demographics }\end{array}$ & -1.023 & 0.399 & 0.360 \\
\hline Gender $^{1}$ & 0.283 & 0.342 & 1.327 & Gender & 0.251 & 0.229 & 1.286 \\
\hline Age $(18-25)^{2}$ & -0.531 & 0.548 & 0.588 & Age $(18-25)^{5}$ & -0.187 & 0.392 & 0.829 \\
\hline Age $(76+)^{2}$ & -3.293 & 1.418 & 0.037 & Age $(66-75)^{5}$ & 0.613 & 0.521 & 1.846 \\
\hline Trip Characteristics & & & & Trip Characteristics & & & \\
\hline Groups & -0.909 & 0.451 & 0.403 & Children & 0.729 & 0.857 & 2.072 \\
\hline Public Transport & 0.249 & 0.395 & 1.283 & Work trip ${ }^{2}$ & 0.161 & 0.274 & 1.174 \\
\hline Work trip ${ }^{3}$ & -1.003 & 0.436 & 0.367 & Eat out trip ${ }^{2}$ & 0.293 & 0.335 & 1.340 \\
\hline Home trip ${ }^{3}$ & 1.460 & 0.868 & 4.307 & Rec trip ${ }^{2}$ & 1.283 & 0.730 & 3.609 \\
\hline Eat out trip ${ }^{3}$ & -0.589 & 0.529 & 0.555 & Exercise trip ${ }^{2}$ & -0.506 & 0.661 & 0.603 \\
\hline Rec trip ${ }^{3}$ & -2.006 & 0.726 & 0.135 & Length $(5-10 \text { mins })^{3}$ & -0.303 & 0.272 & 0.738 \\
\hline Exercise trip ${ }^{3}$ & 0.386 & 0.905 & 1.471 & Length $(10-15 \text { mins })^{3}$ & 0.939 & 0.429 & 2.557 \\
\hline Length (5-10mins) ${ }^{4}$ & 0.499 & 0.433 & 1.647 & Length $(>15 \text { mins })^{3}$ & 0.227 & 0.342 & 1.254 \\
\hline Length $(>15 \text { mins })^{4}$ & -1.005 & 0.432 & 0.366 & Freq (First time) ${ }^{4}$ & 0.729 & 0.516 & 2.073 \\
\hline Freq $(1-3 \text { days/wk })^{5}$ & -0.431 & 0.376 & 0.650 & Freq $(<1 / \mathrm{mo})^{4}$ & 0.619 & 0.445 & 1.857 \\
\hline Location & & & & Location & & & \\
\hline Sandy $/ 43^{\text {rd } 6}$ & 0.645 & 0.475 & 1.905 & Sandy $/ 39^{\text {th }} 6$ & 0.473 & 0.330 & 1.605 \\
\hline Perceptions & & & & Attitudes & & & \\
\hline Delay & 0.137 & 0.478 & 1.146 & CD - Safety & 1.094 & 0.334 & 2.985 \\
\hline Safety & -1.482 & 0.614 & 0.227 & & & & \\
\hline Attitudes & & & & & & & \\
\hline $\mathrm{CD}$ - Compliance & 1.114 & 0.344 & 3.045 & & & & \\
\hline \multicolumn{8}{|c|}{ Overall Model Statistics } \\
\hline$\overline{\mathrm{N}}$ & 362 & & & 362 & & & \\
\hline -2 Log-likelihood & 242.526 & & & 457.819 & & & \\
\hline Model $\chi^{2}$ & $\mathbf{5 5 . 4 2 0}$ & & & 31.170 & & & \\
\hline & & & & & & & \\
\hline Cox \& Snell $\mathrm{R}^{2}$ & 0.142 & & & 0.083 & & & \\
\hline Nagelkerke $\mathrm{R}^{2}$ & 0.253 & & & 0.111 & & & \\
\hline
\end{tabular}


Table 3-8 Models of Delay Satisfaction in Actuated and Recall Intersections

\begin{tabular}{|c|c|c|c|c|c|c|c|}
\hline \multicolumn{4}{|c|}{ Actuated Intersections } & \multicolumn{4}{|c|}{ Recall Intersections } \\
\hline & $\mathbf{B}$ & S.E. & OR & & $\mathbf{B}$ & S.E. & $\overline{\mathbf{O R}}$ \\
\hline Intercept & -1.174 & 0.870 & 0.309 & Intercept & 3.190 & 0.958 & 24.294 \\
\hline Demographics & & & & Demographics & & & \\
\hline Gender $^{1}$ & -0.461 & 0.485 & 0.630 & Gender ${ }^{1}$ & -0.300 & 0.590 & 0.741 \\
\hline Age $(26-39)^{2}$ & 0.254 & 0.523 & 1.290 & Age $(26-39)^{2}$ & -0.548 & 0.608 & 0.578 \\
\hline Age $(66-75)^{2}$ & -0.585 & 1.044 & 0.557 & Age $(66-75)^{2}$ & 1.082 & 1.458 & 2.951 \\
\hline Trip Characteristics & & & & Trip Characteristics & & & \\
\hline School trip ${ }^{3}$ & -1.513 & 1.140 & 0.220 & Groups & 1.330 & 1.118 & 3.783 \\
\hline Home trip ${ }^{3}$ & 1.772 & 0.915 & 5.880 & Public Transport & -1.130 & 0.622 & 0.323 \\
\hline Rec trip ${ }^{3^{2}}$ & 1.696 & 1.176 & 5.454 & Home trip ${ }^{3}$ & -0.662 & 0.734 & 0.516 \\
\hline Exercise trip ${ }^{3}$ & 0.672 & 1.090 & 1.957 & Rec trip ${ }^{3}$ & -2.201 & 1.371 & 0.111 \\
\hline Length $(10-15 \text { mins })^{4}$ & 1.596 & 1.126 & 4.933 & Exercise trip ${ }^{3}$ & -1.515 & 1.508 & 0.220 \\
\hline Length ( $>15$ mins $)^{4}$ & 0.561 & 0.599 & 1.753 & Length (5-10 mins) ${ }^{4}$ & -1.178 & 0.661 & 0.308 \\
\hline Freq $(<1 \text { day } / \mathrm{mo})^{\mathbf{5}}$ & 1.454 & 0.881 & 4.279 & Length $(>15 \text { mins })^{4}$ & -1.005 & 0.840 & 0.366 \\
\hline Freq (1-3 days/wk $)^{5}$ & 0.836 & 0.540 & 2.306 & Freq (1-3 days/mo $)^{5}$ & 1.194 & 1.197 & 3.300 \\
\hline Location & & & & Freq (1-3 days/wk $)^{5}$ & -0.516 & 0.708 & 0.597 \\
\hline Multnomah/13th ${ }^{6}$ & -0.732 & 0.501 & 0.481 & Location & & & \\
\hline Perceptions & & & & Multnomah/9th ${ }^{7}$ & -0.379 & 0.620 & 0.685 \\
\hline Safety & 1.697 & 0.580 & 5.455 & Perceptions & & & \\
\hline Attitudes & & & & Safety & 1.977 & 0.595 & 7.222 \\
\hline \multirow{2}{*}{ CD-Safety } & 0.866 & 0.601 & 2.377 & Attitudes & & & \\
\hline & & & & CD-Compliance & -0.444 & 0.597 & 0.641 \\
\hline \multicolumn{8}{|c|}{ Overall Model Statistics } \\
\hline $\mathrm{N}$ & 132 & & & 231 & & & \\
\hline -2 Log-likelihood & 126.153 & & & 99.861 & & & \\
\hline Model chi-square & 24.460 & & & 26.572 & & & \\
\hline & 14 & & & 15 & & & \\
\hline Cox \& Snell $\mathrm{R}^{2}$ & 0.169 & & & 0.109 & & & \\
\hline Nagelkerke $\mathrm{R}^{2}$ & 0.249 & & & 0.258 & & & \\
\hline
\end{tabular}

*Bolded coefficients are significant at $\mathrm{p}<0.05$. ${ }^{* *}$ Bolded and italicized coefficients are significant at $\mathrm{p}<0.1$.

${ }^{1}$ Male - base case, ${ }^{2}$ Age (40-65) - base case, ${ }^{\mathbf{3}}$ Shopping trip - base case, ${ }^{4}$ Length $\left(<5\right.$ mins) - base case, ${ }^{5}$ Frequency ( 4 or more days per week) - base case, ${ }^{6}$ Sandy $/ 39$ th - base case, ${ }^{7}$ Sandy $/ 43^{\text {rd }}-$ base case. 
Table 3-9 Corridor Level Models of Satisfaction with Delay

\begin{tabular}{|c|c|c|c|c|c|c|c|}
\hline \multicolumn{4}{|c|}{ Multnomah } & \multicolumn{4}{|c|}{ Sandy } \\
\hline & B & S.E. & OR & & B & S.E. & OR \\
\hline Intercept & 0.269 & 0.821 & 1.309 & Intercept & -0.057 & 1.395 & 0.944 \\
\hline Demographics & & & & Demographics & & & \\
\hline Gender $^{1}$ & -0.186 & 0.429 & 0.830 & Gender $^{1}$ & 0.867 & 0.774 & 2.379 \\
\hline Age $(18-25)^{2}$ & -1.175 & 0.671 & 0.309 & Age $(26-39)^{2}$ & -0.843 & 0.806 & 0.431 \\
\hline Local & -0.524 & 0.591 & 0.592 & Age $(66-75)^{2}$ & -0.592 & 1.195 & 0.553 \\
\hline Trip Characteristics & & & & Local & 0.036 & 0.919 & 1.037 \\
\hline Groups & 0.410 & 0.644 & 1.506 & Trip Characteristics & & & \\
\hline Public Transport & 0.574 & 0.485 & 1.775 & Work trip ${ }^{3}$ & -1.174 & 0.875 & 0.309 \\
\hline Work trip ${ }^{3}$ & 0.256 & 0.513 & 1.291 & Home trip ${ }^{3}$ & -0.556 & 1.049 & 0.574 \\
\hline Home trip ${ }^{3}$ & 0.374 & 0.757 & 1.453 & Eat out trip ${ }^{3}$ & 0.790 & 1.364 & 2.204 \\
\hline Other ${ }^{3}$ & -2.291 & 1.647 & 0.101 & Length $(5-10 \text { mins })^{4}$ & -2.752 & 0.952 & 0.064 \\
\hline Length (10-15 mins) ${ }^{4}$ & 0.990 & 0.860 & 2.692 & Length $(>15 \text { mins })^{4}$ & -1.614 & 1.079 & 0.199 \\
\hline Freq $(<1 / \mathrm{mo})^{5}$ & 1.309 & 0.915 & 3.702 & Freq (1-3 days/mo $)^{5}$ & -1.421 & 0.994 & 0.241 \\
\hline Freq $(1-3 \text { days } / \mathrm{mo})^{5}$ & 0.789 & 0.750 & 2.200 & Freq (1-3 days/wk $)^{5}$ & 2.830 & 1.355 & 16.941 \\
\hline Location & & & & Location & & & \\
\hline Recall Intersection ${ }^{6}$ & 1.646 & 0.458 & 5.188 & Recall Intersection $^{7}$ & 1.951 & 0.753 & 7.033 \\
\hline Perceptions & & & & Perceptions & & & \\
\hline Safety & 1.017 & 0.490 & 2.764 & Safety & 3.379 & 0.936 & 29.342 \\
\hline Attitudes & & & & Attitudes & & & \\
\hline CD -Compliance & -0.377 & 0.444 & 0.686 & CD - Safety & 0.731 & 1.052 & 2.077 \\
\hline \multicolumn{8}{|c|}{ Overall Model Statistics } \\
\hline $\mathrm{N}$ & 217 & & & 146 & & & \\
\hline -2 Log-likelihood & 155.084 & & & 65.072 & & & \\
\hline Model chi-square & 33.327 & & & 43.968 & & & \\
\hline & 14 & & & 14 & & & \\
\hline Cox \& Snell $\mathrm{R}^{2}$ & 0.142 & & & 0.260 & & & \\
\hline Nagelkerke $\mathrm{R}^{2}$ & 0.245 & & & 0.494 & & & \\
\hline
\end{tabular}

*Bolded coefficients are significant at $\mathrm{p}<0.05$. ${ }^{*}$ Bolded and italicized coefficients are significant at $\mathrm{p}<0.1$.

${ }^{1}$ Male - base case, ${ }^{2}$ Age (40-65) - base case, ${ }^{3}$ Shopping trip - base case, ${ }^{4}$ Length $\left(<5\right.$ mins) - base case, ${ }^{\mathbf{5}}$ Frequency (4 or more days per week) - base case, ${ }^{6}$ Multnomah/13th - base case, ${ }^{7}$ Sandy $/ 39^{\text {th }}-$ base case.

Table 3-9 presents the results of the corridor level models. For the intersections along Multnomah St. significant predictors for satisfaction with delay included location, perception of safety and young adults. Respondents at Multnomah and $9^{\text {th }}$ (recall intersection) were $419 \%$ higher odds of being satisfied with delay compared to the respondents at Multnomah and $13^{\text {th }}$ (actuated intersection), controlling for cycle length. Respondents who perceived the intersections along this corridor as safe were associated with $176 \%$ higher odds of satisfaction with delay. As expected, young adults had $70 \%$ 
lower odds of being satisfied with delay than older adults (40-65). Along Sandy Blvd. the findings were similar; respondents at Sandy $\& 43^{\text {rd }}$ (recall intersection) had $603 \%$ higher odds of being satisfied with delay compared to respondents at the actuated intersection along the same corridor. Also significant were trip frequency of 1-3 days per week and trip length of 5-10 minutes. These findings indicated that respondents whose trip length was between 5-10 minutes had 93\% lower odds of being satisfied with delay compared to respondents on short trips $(<5$ minutes $)$.

\subsection{Discussion and Summary}

This exploratory study examined factors that influence attitudes and pedestrian perceptions of safety, delay and determinants of crossing behaviors at signalized intersections. It adds to the body of work related to pedestrian behavior and its relation to safety. The empirical analysis was based on an intercept survey conducted at four signalized intersections in Portland, OR. The survey results reveal that for most individuals, both safety and compliance play a role in crossing decisions. However, more respondents cited that safety was a concern than compliance while making a decision to cross. Although we did not directly observe noncompliance in this study, the results suggest that individuals may perceive safety to be the greater threat and may be willing to violate the signal indication based on their assessment of risks. An individual's assessment of risk is considered to be highly subjective and varies significantly among the population. The lack of enforcement at these intersections may also play a role in respondents' crossing decisions and their assessment of risk. The lack of pedestrian 
enforcement, in general, may play a role in non-compliance on a larger scale. Education measures such as increasing awareness of the consequences of unsafe behavior and promoting safe crossing strategies can help in encouraging safe road use. Targeted enforcement can also promote signal compliance.

The findings also reveal that an individual's sensitivity to time is a factor in crossing decisions. Respondents on trips, who are time constrained (work-based trip, trips of longer duration) were less concerned about safety while crossing. These findings are in line with other studies that have shown that trip purpose is a significant factor for compliance and/or crossing speed (Ishaque et al., 2008, Wang et al., 2011). Respondents crossing in groups were also less likely to consider safety issues perhaps due to the influence of group behavior. Previous studies have found similar trends with respect to group behavior; pedestrians approaching the intersection are likely to follow the lead of others in either complying or violating the signal indication (Yanfeng et al., 2010, Bradbury et al., 2012 and Wang et al., 2011). Interventions targeted towards encouraging desirable and safe group behaviors can be beneficial for increasing pedestrian safety.

The survey findings also revealed that satisfaction with delay was correlated with time constraints. Respondents using public transportation and on trips of short duration were dissatisfied with delay. Operational improvements to make the signals more responsive towards pedestrians during peak periods, at intersections that are located close to transit stops can assist in promoting compliance. Younger adults also expressed dissatisfaction with delay; targeted intervention aimed at young adults to increase their knowledge and awareness regarding road safety will help in promoting safe crossing 
behaviors. This finding is consistent with other studies (Wang et al., 2011, Diaz, 2002).

The type of pedestrian detection at signalized intersections also influenced the perception of delay; respondents at intersections that were placed on recall were more satisfied with delay than respondents at actuated intersections. This is perhaps related to the additional burden placed on the respondent at actuated intersections, to activate a push button in order to be served. Many intersections equipped with push buttons offer no feedback to the pedestrian that their call for service was received, which may lead to increased frustration. Previous research by Van Houten et al. showed that installing pushbuttons that provided visual and auditory feedback resulted in increased compliance (Van Houten et al., 2006). Accessibility of push buttons is also critical. Operational improvements at actuated intersections such as installing clear signage indicating the necessity to activate the push button in order to be served, providing auditory or visual feedback, increasing accessibility and taking measures to reduce pedestrian delay will help in reducing dissatisfaction with delay.

There were a few limitations and potential biases with our study. Older adults were not sufficiently captured in our sample and children were not surveyed in our study. Also, older adults with hearing and sight limitations were unable to take the survey. Since these groups are the most vulnerable of the crossing population and given that crossing behaviors and attitudes vary by age, future work should include these populations. As the survey was only administered in English, non-English speaking populations were not included in our survey. In the future, surveys should be administered in multiple languages to obtain a representative sample. While this study obtained stated perceptions 
and attitudes, actual crossing behavior was not observed. A future study comparing stated preferences and revealed behavior with respect to pedestrian crossing decisions might be beneficial. Finally, this survey should be expanded to more locations with different land use patterns, varying built environment features, different weather conditions and varying time periods to gain a better understanding of pedestrian behavior.

The survey findings illustrate the complexity of crossing decisions. In addition to demographics, trip characteristics, attitudes and perceptions, operational decisions at intersections also influence crossing behavior, primarily through the delays imposed by the signal timing policies on pedestrians. Efficiency at intersections is often quantified based on level of service measures, which include delay as a critical component. While vehicle delays have been well researched, research on pedestrian delay has been lagging. The next chapter outlines the current state of research with respect to pedestrian delay estimation and describes efforts to automatically measure pedestrian delay at the intersections using signal controllers. 


\section{MEASURING PEDESTRIAN DELAY}

The findings from the intercept survey described in Chapter 3 revealed that the waiting times experienced by pedestrians at an intersection play a role in their crossing behavior. In this research, pedestrian delay is defined as the difference between the time when the pedestrian activates the push button and the time that the pedestrian phase is served. Delay is an important performance metric from the traffic operations perspective. While the goal of traditional signal timing is to minimize vehicular delay, pedestrian delay is not usually considered while designing optimal timing. Though the HCM provides an equation to estimate pedestrian delay, researchers have found deficiencies in the equation with regard to accuracy (Hubbard, 2008, Kothuri et al., 2013) and its inability to account for delay modulation resulting from changes in signal controller parameter setting or mode of operation (Kothuri et al. 2013). This chapter reviews literature on pedestrian delay estimation, measurement and methods to incorporate pedestrian delay during signal timing development and optimization. Methods to directly measure pedestrian delay in the controller using internal logic commands are outlined and impacts of signal controller parameter changes on pedestrian delay are discussed in this chapter.

\subsection{Review of Literature}

Although urban freeways facilities carry significant traffic, it is estimated that $40-50 \%$ of all vehicle miles traveled (Tarnoff 2009, Berkow 2009) occur on arterials. While performance measures on the vehicle front (travel times, delays) are well estimated and researched (Turner 1996, Zhang, 1999, Skabardonis 2008, Liu 2009, Quayle 2010), 
multimodal arterial performance measures have been attracting increased attention only recently. Some early work by Fruin led to the development of LOS measures for pedestrian flow and queuing based on allocations of pedestrian space (Fruin, 1971). More recently, HCM 2010 provides a number of pedestrian performance measures for evaluating intersection performance such as corner and crosswalk circulation areas, pedestrian delay and pedestrian Level of Service (LOS) score. While circulation area refers to the space available to the pedestrian, delay refers to the waiting time experienced by the pedestrian to legally cross the street. The intersection pedestrian LOS score combines a number of variables such as vehicle volume, vehicle speed and pedestrian delay and is calculated using the following equation as outlined in the HCM.

$$
I_{p}=0.5997+F_{w}+F_{v}+F_{s}+F_{\text {delay }}
$$

where,

$\mathrm{I}_{\mathrm{p}}=$ pedestrian LOS score for an intersection

$\mathrm{F}_{\mathrm{w}}=$ cross-section adjustment factor

$F_{v}=$ motorized vehicle volume adjustment factor

$F_{\mathrm{s}}=$ motorized vehicle speed adjustment factor

$\mathrm{F}_{\text {delay }}=$ pedestrian delay adjustment factor

$\mathrm{F}_{\text {delay }}$ is calculated using the following equation.

$$
F_{\text {delay }}=0.0401 \ln \left(d_{p}\right)
$$

where,

$$
d_{p}=\text { average pedestrian delay }(s / p)
$$


The average pedestrian delay for the major street is calculated as

$$
d_{p}=\frac{0.5(c-g)^{2}}{C}
$$

where,

$\mathrm{dp}=$ average pedestrian delay $(\mathrm{s} / \mathrm{p})$ or wait time

$\mathrm{C}=$ cycle length $(\mathrm{s})$

$\mathrm{g}=$ effective walk time for pedestrians $(\mathrm{s})$

The effective walk time is estimated using equations 4-4, 4-5 or 4-6 depending on the type of phase (pretimed vs. actuated) as well as whether rest-in walk is enabled. Rest in walk for a pedestrian phase is a feature which if set, enables the walk display to be maximized during a vehicle green (Koonce et al., 2008).

If the phase is actuated with a pedestrian signal head and rest-in-walk is not enabled or the phase is pre-timed with a pedestrian signal head, then

$$
g=w a l k+4.0
$$

If the phase is actuated and rest in walk is enabled then

$$
g=D_{p}-Y-R_{c}-P C+4.0
$$

If there is no pedestrian signal head,

$$
g=D_{p}-Y-R_{c}
$$

where, 


$$
\begin{aligned}
& \mathrm{g}=\text { effective walk time }(\mathrm{sec}) \\
& \mathrm{W}=\text { walk setting for minor street pedestrian phase }(\mathrm{sec}) \\
& \mathrm{PC}=\text { pedestrian clearance }(\mathrm{sec}) \\
& D_{\mathrm{p}}=\text { phase duration }(\mathrm{sec}) \\
& Y=\text { yellow change interval }(\mathrm{sec}) \\
& \mathrm{R}_{\mathrm{c}}=\text { red clearance interval }(\mathrm{sec})
\end{aligned}
$$

The LOS score calculated using the above equations is used to estimate pedestrian LOS based on ranges outlined in HCM, 2010. These are listed in Table 4-1.

\section{Table 4-1 Pedestrian Intersection LOS Range}

\begin{tabular}{l|r}
\hline \multicolumn{1}{c|}{ LOS } & \multicolumn{1}{c}{ LOS Score } \\
\hline A & $\leq 2.00$ \\
B & $>2.00-2.75$ \\
C & $>2.75-3.50$ \\
D & $>3.50-4.25$ \\
E & $>4.25-5.00$ \\
F & $>5.00$ \\
\hline
\end{tabular}

Equation 4-3 used in the HCM to estimate pedestrian delay was proposed by Pretty for one-stage crossings based on the assumption of uniform arrivals (Pretty 1979). Equation 4-3 also assumes complete pedestrian compliance. Braun and Roddin suggested a modification to include fraction of pedestrians who comply with the signal indication (Braun \& Roddin 1978). Their suggested equation is:

$$
d_{p}=\frac{0.5 F(C-g)^{2}}{C}
$$

where,

$\mathrm{F}=$ fraction of pedestrians who obey the signal. 
The above equation assumes that non complying pedestrians incur no delay. Based on data collected in Brisbane, Australia, Virkler suggested a modification to equation proposed by Pretty (Virkler, 1998). His observations showed that $69 \%$ of the clearance period was used by pedestrians as effective green. The modified equation is shown below:

$$
d_{p}=\frac{0.5(C-g+0.69 A)^{2}}{C}
$$

where,

$A=$ duration of the Flashing Don't Walk or pedestrian clearance interval Prior research has shown that the estimated pedestrian delay using equation 4-3 does not match well with the measured delay (Hubbard et al., 2008, Kothuri et al., 2012). Therefore some agencies are deploying new technology or leveraging existing infrastructure to gather pedestrian actuations and delay (Day et al., 2011, Kothuri et al., 2012). Equation 4-3 listed above assumes that pedestrian delay is only a function of the cycle length and the effective green time. However, actual pedestrian delay is more complicated and depends on certain signal timing parameters such as permissive period, which are not included in the above equations (Kothuri et al., 2013). In addition, there has been no discussion on the mode of operation (coordinated vs. free) and its resulting effect on pedestrian delay.

Tian evaluated different forms of split phasing due to various pedestrian timing treatments (Tian et al., 2001). Protected, permitted, protected/permitted, protected left turn displays with two-stage crossing treatments and an exclusive pedestrian phase were studied and their impacts on coordinated systems were analyzed. The efficiency of 
coordinated signal systems as a result of the various phasing schemes was evaluated. However, pedestrian delay was not explicitly included in the analysis. Wang et al. proposed a pedestrian delay model with a two stage crossing design for unconventional pedestrian crossings (Wang, 2010). While the proposed model provides a method to estimate average pedestrian delay at two stage crossings, it does not propose any strategies to reduce delay at single crossings.

Early signal timing efforts focused on reducing vehicle delay and ignored pedestrian delay (Webster 1958, Little 1975). Bhattacharya and Virkler used the signal timing optimization software Synchro to study the changes in vehicle delay resulting from changes in coordination plans and offsets (Bhattacharya and Virkler, 2005). They found that offsets that produce lowest vehicle delay are not always the same as offsets produced when lowest user cost is desirable and pedestrian value of time in considered (Bhattacharya and Virkler, 2005). Recent research mainly in the United Kingdom has focused on optimizing signal timing based on vehicular and pedestrian delays. Through a simple analytical model, Noland analyzed the travel delay costs of pedestrians and showed that ignoring pedestrian delay and focusing on vehicular flows may not be the most cost effective solution from an economic perspective as the travel time costs of delay to pedestrians may be significant (Noland 2003). Ishaque and Noland used micro simulation to understand pedestrian delay and study the trade-offs between pedestrian and vehicle delays in a hypothetical network (Ishaque and Noland 2005, Ishaque 2006). These studies found that low cycle lengths benefit pedestrians. Different flow combinations of pedestrians and vehicles were tested along with different type of 
pedestrian crossings (single, double and staggered) and a matrix identifying the proposed pedestrian phase based on the proportion of pedestrians and vehicles was developed. These results were based on models calibrated using traffic parameters and driving behavior in England and as such may not be directly transferrable to the U.S. Also, while the type of pedestrian crossing was varied, these studies did not look at either the effects of changing the mode of operation or the signal timing parameters on pedestrian delay.

Research conducted in New Zealand proposed methods to reduce pedestrian delay using micro-simulation modeling in three cities (Vallyon and Turner, 2011). Strategies evaluated included phasing changes, signal timing optimization and cycle length reduction. Per person optimization of time was proposed instead of per vehicle to allow for equitable consideration of all users. Although this study evaluated the impacts of certain pedestrian control strategies on delay, it did not evaluate the effects of mode of signal controller operation on delay and the associated feasibility regimes. Roshandeh et al. proposed simultaneous minimization of vehicle and pedestrian delays by adjusting green splits during the peak periods and timing plans during other time periods in a day, without changing cycle lengths and signal coordination (Roshandeh et al., 2013). While their signal timing optimization reduced delays within the coordinated framework; delays resulting from uncoordinated operation were not considered.

\subsection{Pedestrian Delay Measurement}

The City of Portland operates more than 1,050 signalized intersections, with a mix of Type 170 and 2070 controllers. Type 2070 controllers are newer and are operated using 
Voyage controller software provided by Northwest Signal Supply, Inc. (Northwest Signal Supply Inc., 2008). Voyage software can be used at isolated intersections or as a larger part of the central control system (Northwest Signal Supply Inc., 2008). Two methods were developed to automate the process for pedestrian delay data collection using Voyage software. Both the methods relied on pedestrian push button actuations to capture delay. Direct measurement of pedestrian delay using existing resources provides the system operators and engineers with data, that they could use to make better operational decisions at the intersections to serve pedestrians more efficiently.

\subsection{Methodology}

Along major arterial corridors in the City of Portland, pedestrian recall is implemented on the mainline phases (2 and 6), which ensures that pedestrians crossing concurrently with the main line get served each cycle, irrespective of demand. Thus, the relative importance of measuring delay on these movements is less than the more variable cross street travel. The pedestrian delay data in this study are only collected for side street pedestrian phases that are pedestrian actuated with push buttons. As detailed in section 2.2, NEMA has adopted specific phase numbering standards (1-8) for defining phase movements. Through and left turning movements are represented by even and odd numbers respectively. Figure 4-1 shows the phase diagram for the intersection of NE $82^{\text {nd }}$ Avenue and NE Tillamook Street in Portland, OR. This intersection operates as a 6 phase intersection, with phases 2 and 6 serving the mainline through movements (NE $82^{\text {nd }}$ Avenue south and north bound). As described previously, pedestrian phases P2 and P6 
are on recall. Methods to measure delay were implemented for pedestrian phases P4 and P8 (crossing the major arterial NE $82^{\text {nd }}$ Avenue), which are not on recall and are equipped with push buttons. A description of the two methods used for collecting pedestrian waiting times is provided below.

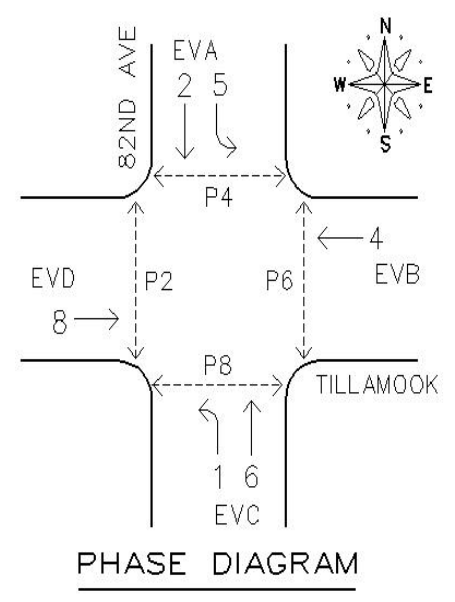

Figure 4-1 Phase Diagram for NE 82nd Avenue and NE Tillamook Street (Source: Portland Bureau of Transportation)

\subsubsection{Transit Priority Logging}

Among the various advanced features present in the Voyage software is the ability to implement transit priority. At various signalized intersections, the City of Portland implements conditional transit priority if a bus is running late to either "extend the green phase" or "shorten the red phase" depending on the time in the cycle during which a bus arrives at an intersection (Byrne et al., 2005). The transit priority log in the Voyage software records the activation time (when the transit priority call was received) and the time of service (when the call was served) for each event. Using the transit priority logging capability, logic was implemented in Voyage software to capture pedestrian delay for the first actuation in each cycle, by logging a pedestrian call as a transit priority 
call. To record waiting time for pedestrians, we log the time when a pedestrian call is placed and when it gets served. At intersections, where transit priority for buses is active, a sequence is needed to inform the controller regarding the order of priority for the two events. Currently, priority is set equal implying that the calls are handled on a first come first serve basis. Figure 4-2 shows a graphic of the logic used to capture delay for a pedestrian movement.

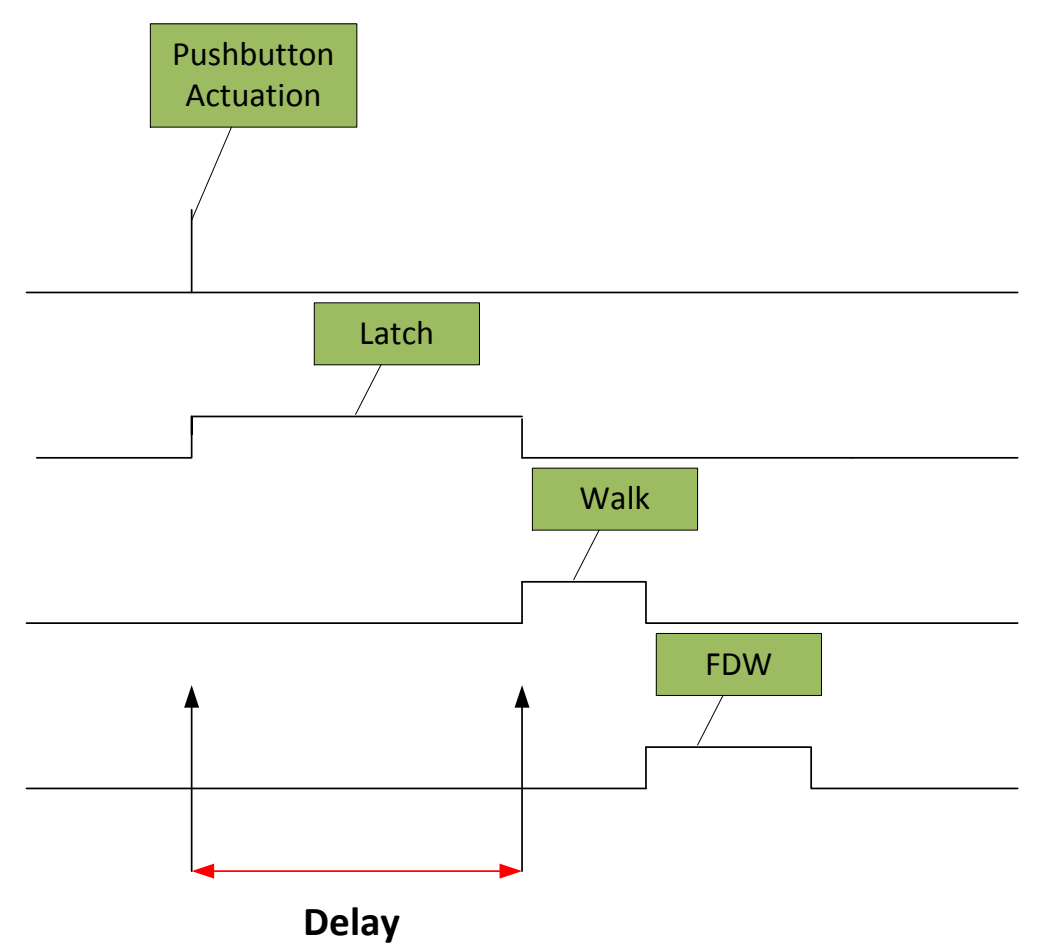

Figure 4-2 Pedestrian Actuation Delay using Transit Priority Logs

The pedestrian push button is reassigned to a vehicle detector and the logic is set up in a way such that a latch is turned on if the pushbutton is activated and the walk phase is not active currently. The latch ensures that the transit priority input turns on and is only released when the walk is served. Additional pedestrian calls during the walk phase are ignored by the logging feature. This allows the system to keep track of the time the push 
button was activated and the time until that the walk phase was served. One primary limitation of this method is that only one transit priority event can be active at a particular time. This limitation can hinder the actual transit priority operation, if the priority sequence is not set correctly. In addition, at an intersection if there are two pedestrian actuated phases and if the push buttons corresponding to both phases are actuated at the same time, only one pedestrian actuation event will be active and recorded at a particular time. Future versions of Voyage software will mitigate this issue by collecting delay as a standard measure of effectiveness (MOE) in the controller.

\subsubsection{Volume Bin Logging}

In addition to the using transit priority logs, another method using internal timers in the controller was also implemented to record pedestrian delay, which is grouped into bins. Three bins are used for delay data collection: 0-20 sec, $20-40 \mathrm{sec}$ and $>40 \mathrm{sec}$. Figure 4-3 shows the logic used in the implementation of this method. The dashed red lines indicate bin boundaries and the dashed dot blue line indicates when the walk is served for each case. When a pedestrian call is received, a latch is set using internal logic commands in Voyage software. Four (4) timers per each pedestrian phase are activated when the latch is set, the first timer counts down from $20 \mathrm{sec}$ to 0 (timers 5/6), the second timer counts down from $40 \mathrm{sec}$ to 0 (timers 7/8), the third timer counts down from $0.1 \mathrm{sec}$ to 0 when the walk is served (timers $1 / 2$ ) and the fourth timer counts down from $1 \mathrm{sec}$ to 0 when the walk ends and the clearance interval begins (timers 3/4). While the first and second 
timers classify the delay into the respective bins, the third and fourth timers keep track of beginning and end of walk indication.

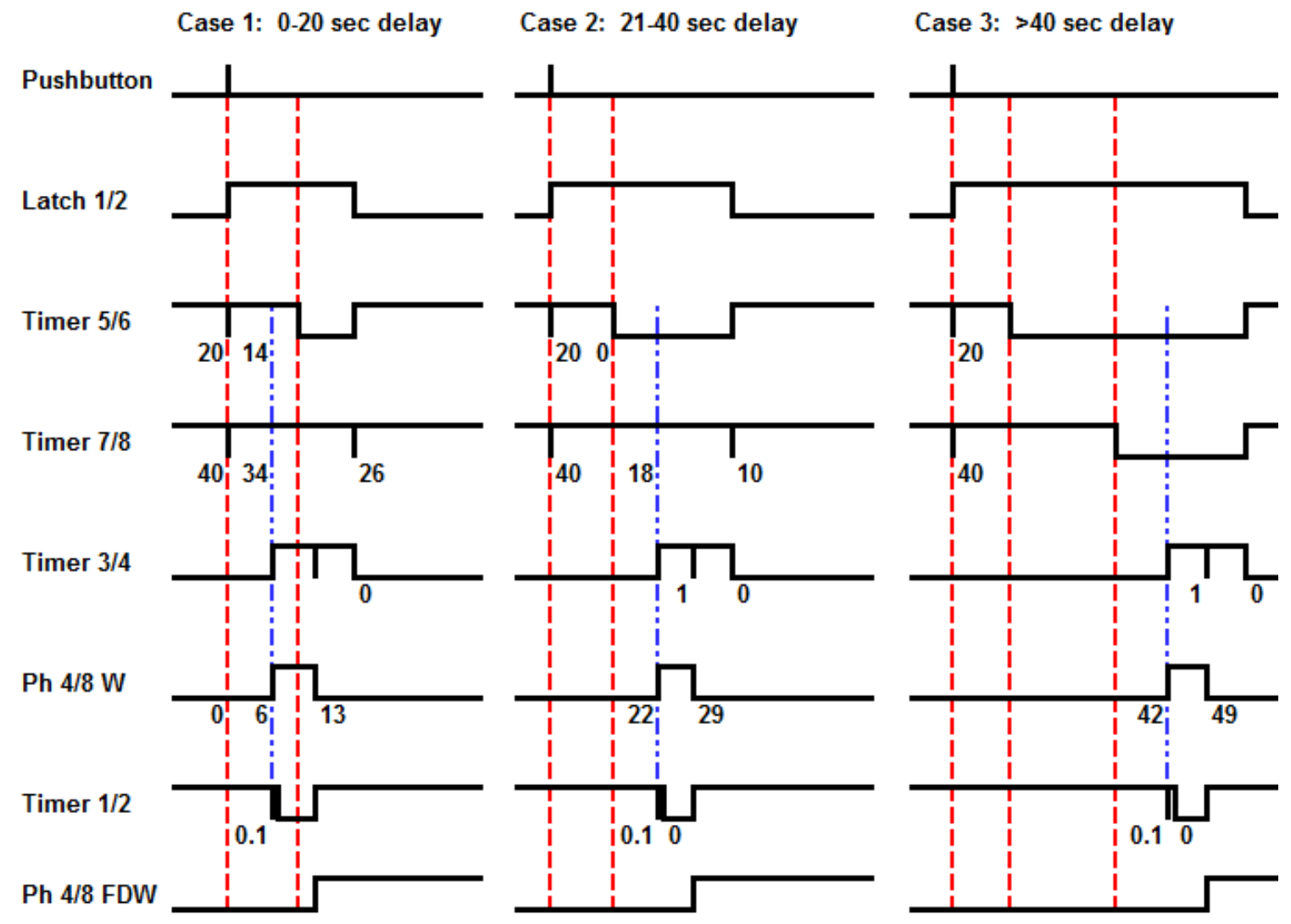

Figure 4-3 Pedestrian Delay using Volume Logs

The logic for classifying delay into bins is shown below: For each bin, the criteria listed below have to be satisfied in order for the delay value to be placed in that bin.

Bin 1: Delay between 0 - 20 sec
a. Latch 1 is set
b. Walk indication is active
c. Timer $1 \neq 0$ 
d. Timer $5 \neq 0$

Bin 2: Delay between $20-40$ sec
a. Latch 1 is set
b. Walk indication is active
c. Timer $1 \neq 0$
d. Timer $7 \neq 0$
e. Timer $5=0$

Bin 3: Delay greater than 40 sec
a. Latch 1 is set
b. Walk indication is active
c. Timer $1 \neq 0$
d. $\quad$ Timer $7=0$

Each bin is reassigned to a vehicle detector, so that the counts (delay) from these bins can be obtained through the volume logs in TransSuite $\AA$, the central signal system database used by the City of Portland. While this method provides less detailed (delays are binned rather than reporting actual time) data than the transit priority log method, it is considered more accurate due to the inability of the transit priority log method to handle multiple pedestrian calls or the introduction of incorrect data when a bus transit priority event occurs at the same time. 


\subsection{Pedestrian Delay Data}

The internal logic commands described above have been implemented at approximately twenty intersections in the City of Portland. The criteria for implementation are: presence of 2070 signal controller and availability of internal logic commands. The Voyage signal controller software allows up to 255 internal logic commands per intersection. In addition to delay measurement, internal logic commands are also used to implement advanced traffic control features at intersections.

Table 4-2 shows raw data obtained from the transit priority log for NE $82^{\text {nd }}$ Avenue and NE Tillamook Street. The transit priority column indicates the priority input at the intersection. At this intersection, bus transit priority is not active; transit priority inputs 2 and 4 indicate delay data logging for pedestrian phases 4 and 8 respectively. The status message "TP Input Active" indicates pedestrian push button actuation and "TP Phases Achieved" implies that the walk was served. The difference in times between these two indications is the delay experienced by the pedestrian. Other important data at the time of actuation such as the active phases when the pushbutton was actuated, the active coordination plan, the cycle length, the reading of the cycle timer when the button was pushed, green indication was served and cycle timer reading when the phase terminates are also recorded. 
Table 4-2 Transit Priority Log with Pedestrian Delay Data

\begin{tabular}{|c|c|c|c|c|c|c|c|c|c|}
\hline Date & Time & $\mathbf{T P}$ & Status & $\begin{array}{c}\text { Phases } \\
\text { Active }\end{array}$ & $\begin{array}{c}\text { Coord } \\
\text { Plan }\end{array}$ & $\mathbf{C L}$ & On & Green & Off \\
\hline $7 / 12 / 11$ & $17: 57: 38$ & 2 & TP Input Active & 26 & 3 & 80 & 33 & 0 & 0 \\
\hline $7 / 12 / 11$ & 17:58:29 & 2 & TP Phases Achieved & 48 & 3 & 80 & 33 & 4 & 0 \\
\hline $7 / 12 / 11$ & $17: 58: 29$ & 2 & TP Input Went Inactive & 48 & 3 & 80 & 33 & 4 & 4 \\
\hline $7 / 12 / 11$ & 18:01:52 & 4 & TP Input Active & 26 & 3 & 80 & 47 & 0 & 0 \\
\hline $7 / 12 / 11$ & 18:02:29 & 4 & TP Phases Achieved & 48 & 3 & 80 & 47 & 4 & 0 \\
\hline $7 / 12 / 11$ & $18: 02: 29$ & 4 & TP Input Went Inactive & 48 & 3 & 80 & 47 & 4 & 4 \\
\hline
\end{tabular}

The delay data obtained from the volume logs are less detailed. For a given time period, the count in each bin represents the number of times the delay was within that range. Summing the counts across all bins over the entire day provided the number of actuations for each intersection. Figure 4-4 shows the location of intersections along $82^{\text {nd }}$ Avenue, where pedestrian actuation and delay data were logged using the existing signal controllers. Figure 4-5 shows the pedestrian actuation activity at the eight intersections for one day. Five intersections (SE Division, SE Flavel, SE Holgate, NE Tillamook and SE Woodward) had both phases 4 and 8 active, which implied that each crosswalk crossing $82^{\text {nd }}$ Avenue had a separate pedestrian phase associated with it. Three intersections (SE Boise, SE Mill and NE Wasco) had only pedestrian phase 4 operational, indicating that both crosswalks were tied to the same pedestrian phase.

It is evident from Figure 4-5 that certain intersections (SE Division, SE Holgate and SE Flavel) experience higher pedestrian actuations than the other intersections. The 
higher actuations were observed at intersections which had larger number of destinations surrounding the intersections. The number of actuations per day ranged from a maximum of 548 for SE Holgate to a minimum of 146 at NE Wasco. The availability of the pedestrian actuation data also allows the study of trends.

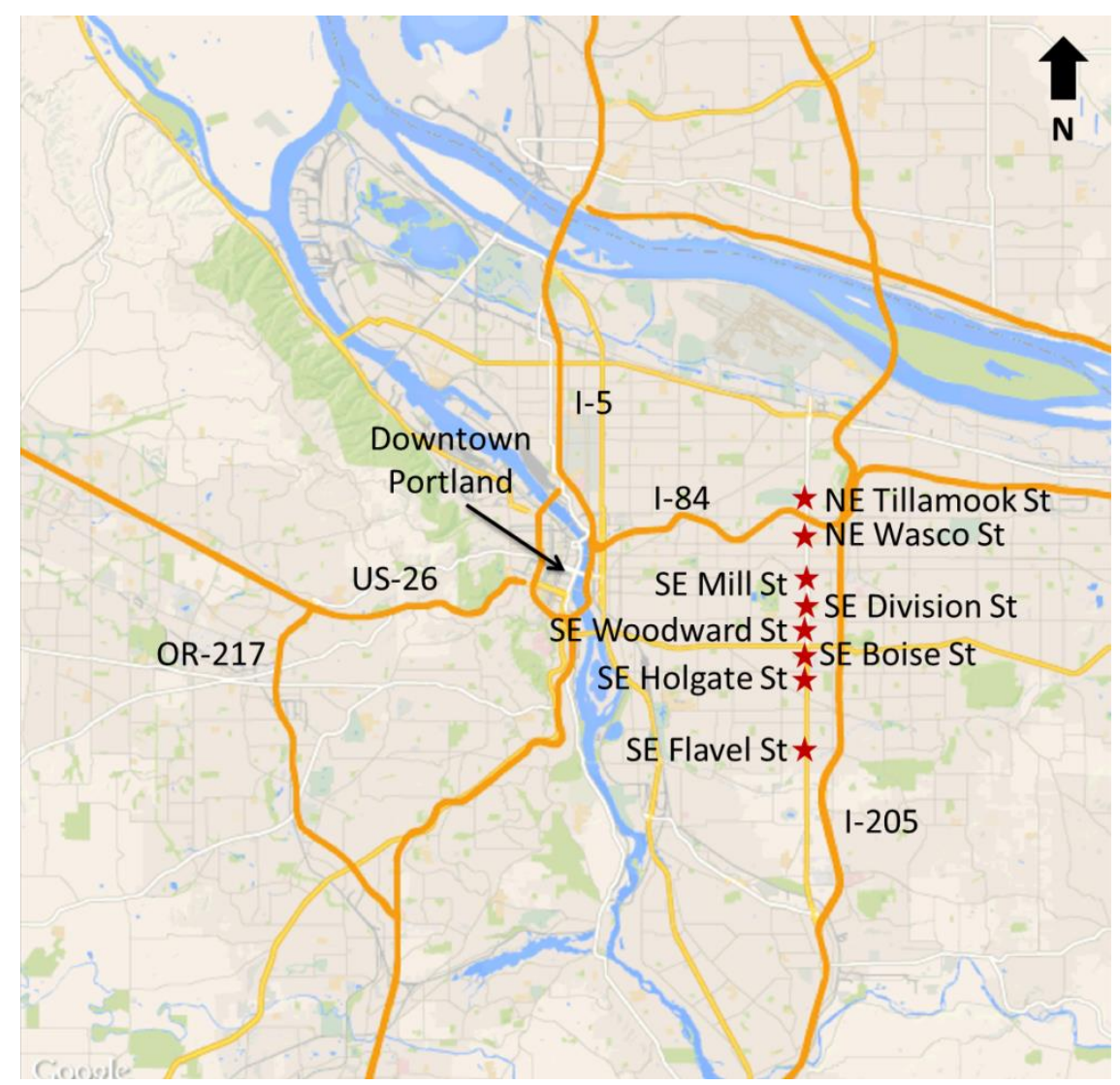

Figure 4-4 Map of Intersections with Actuation and Delay Data 


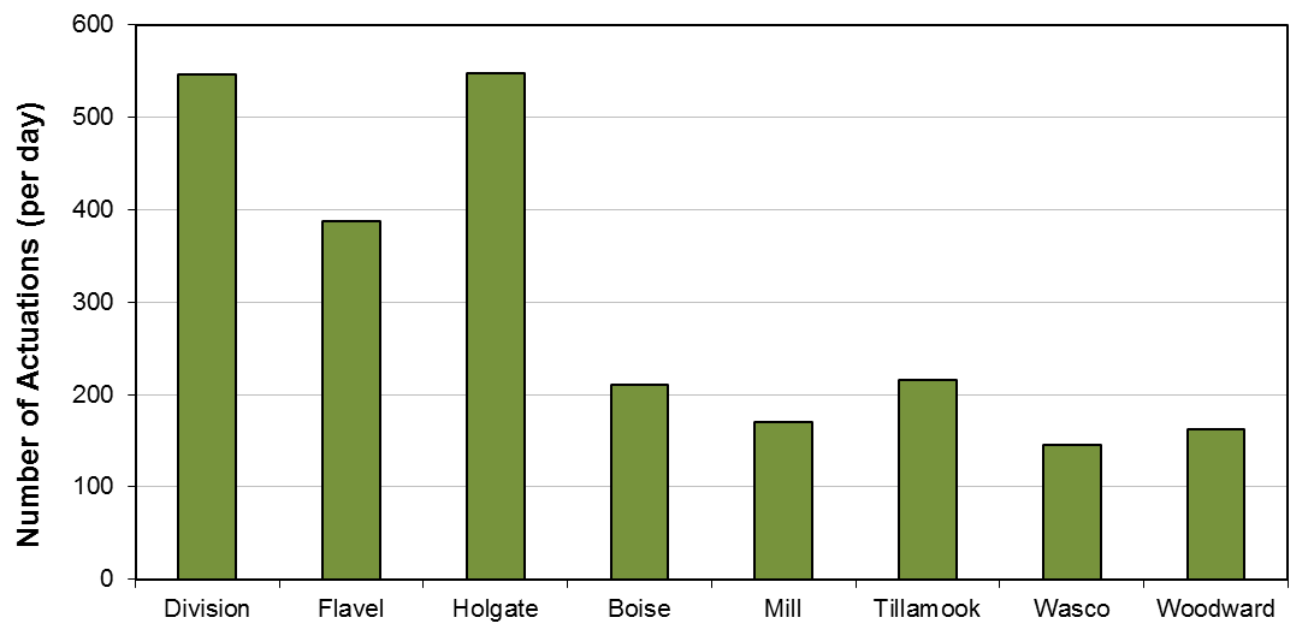

Figure 4-5 Pedestrian Activity across Intersections on Monday, July 18, 2011

Figure 4-6 shows the number of actuations for the intersection of SE $82^{\text {nd }}$ Avenue $\&$ SE Division Street for one week $(07 / 15-07 / 21)$ in July 2011. The plot shows an expected trend of higher weekday actuations and lower weekend actuations. Weekday actuations are consistent Monday through Thursday and trend lower on Friday, during the analyzed time period.

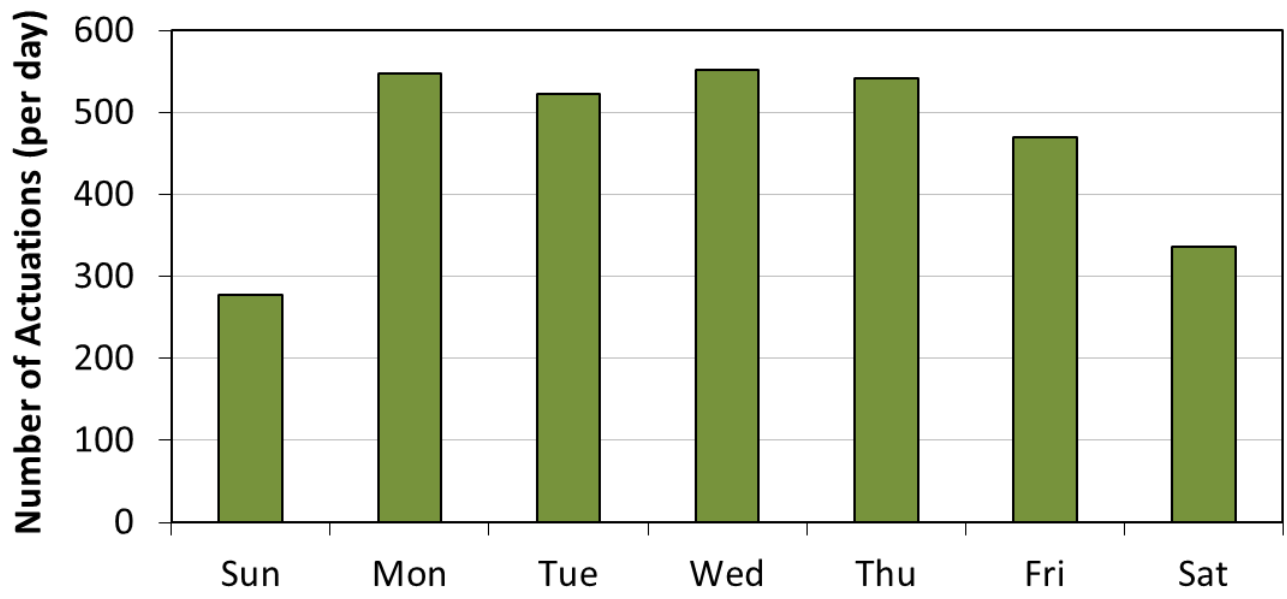

Figure 4-6 Pedestrian Actuations at SE 82nd and SE Division (July 15 -21, 2011) 
At intersections where both pedestrian phases are active for crossing the main street, actuation data can be recorded separately for each phase. Figure 4-7 shows the pedestrian actuations separated by phases. The actuations are for pedestrian phases 4 and 8 for Tuesday, July 19, 2011 at the intersection of $82^{\text {nd }}$ Avenue and Division Street. The plot for phase 4 shows little or no activity at night, higher actuations during AM peak, midday and PM peak periods. The plot for phase 8 on the right shows highest actuations during midday followed by AM and PM peak periods.

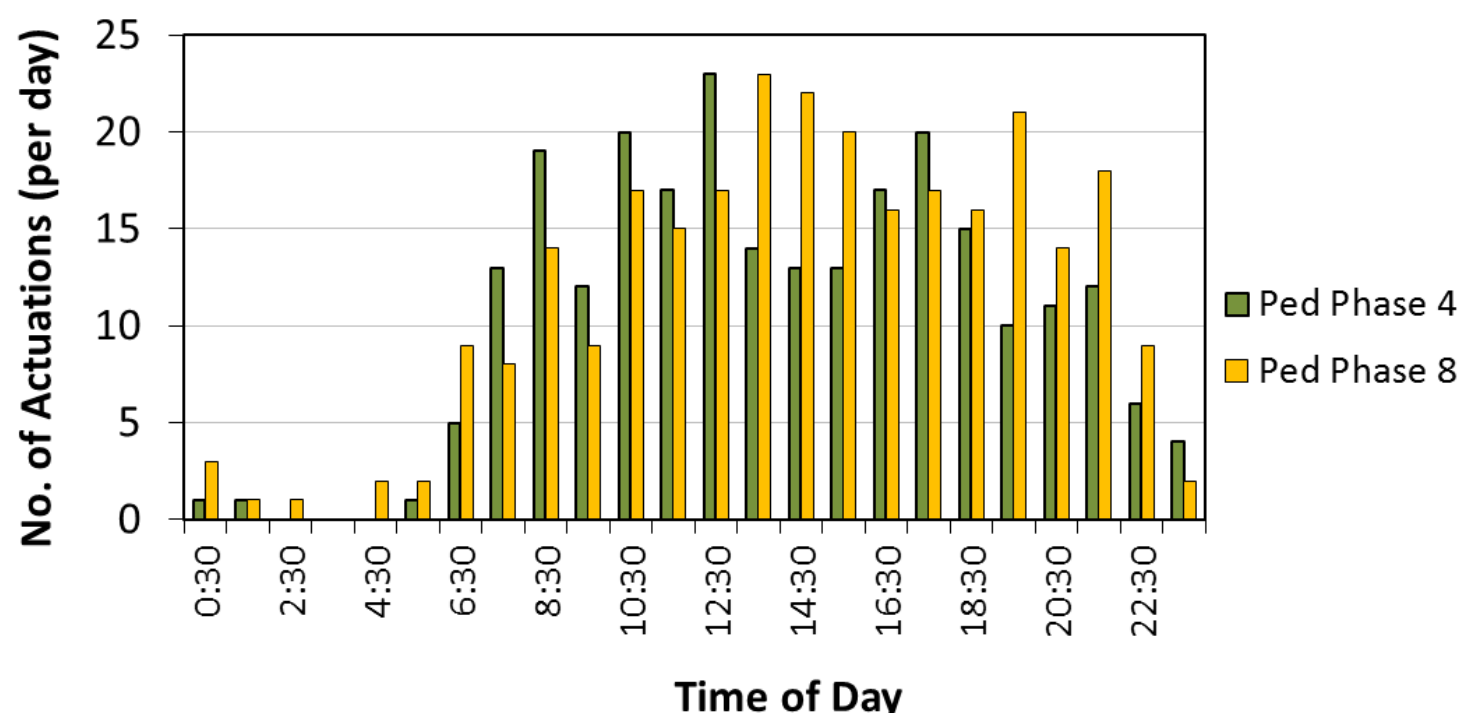

Figure 4-7 Pedestrian Actuations by Phase at SE 82 ${ }^{\text {nd }}$ and SE Division on July 19, 2011

Since the individual actuation data are not available with the bin method, an average delay value for each intersection cannot be estimated directly. However, with some simple assumptions it is possible to estimate the maximum and minimum ranges of the average delay per actuation. Assigning the binned counts to either the maximum or 
minimum delays possible and assuming the maximum delay is equal to the cycle length results in the following equations:

$$
\begin{gathered}
\text { Total Minimum Delay }=0 * C_{1}+21 * C_{2}+41 * C_{3} \\
\text { Total Maximum Delay }=20 * C_{1}+40 * C_{2}+C L * C_{3} \\
\text { Max Average Delay }=\frac{\text { Total Max Delay }}{\left(C_{1}+C_{2}+C_{3}\right)} \\
\text { Min Average Delay }=\frac{\text { Total Min Delay }}{\left(C_{1}+C_{2}+C_{3}\right)} \\
\text { Average Delay for Intersection }=(\text { Min Average Delay, Max Average Delay })
\end{gathered}
$$
where,

$$
\begin{aligned}
& \text { Count in bin } 1=\mathrm{C}_{1} \\
& \text { Count in bin } 2=\mathrm{C}_{2} \\
& \text { Count in bin } 3=\mathrm{C}_{3} \\
& \text { Cycle Length }=\mathrm{CL}
\end{aligned}
$$

Table 4-3 shows the sample calculations for the ranges of delay for one day $(7 / 25 / 2011)$ at five intersections as well as comparison of the two methods for three intersections. The delays obtained from transit priority logging method were classified into bins and compared to the delay from the volume bins. The results indicate that the TP method records fewer actuations compared to the volume bin method. The difference arises due to the ability of the transit priority logger to record only one event at a time, as described previously. 
Table 4-3 Average Delay per Actuation

\begin{tabular}{|c|c|c|c|c|c|c|c|c|c|}
\hline \multirow{3}{*}{ Intersection } & \multicolumn{6}{|c|}{ Counts } & \multicolumn{3}{|c|}{ Delays per actuation (s) } \\
\hline & \multicolumn{2}{|c|}{ Bin 1} & \multicolumn{2}{|c|}{ Bin 2} & \multicolumn{2}{|c|}{ Bin 3} & \multirow{2}{*}{$\begin{array}{l}\text { TP } \\
\text { Avg }\end{array}$} & \multicolumn{2}{|c|}{ Vol } \\
\hline & TP & Vol & TP & Vol & TP & Vol & & Min & Max \\
\hline NE $82^{\text {nd }} \&$ NE Tillamook & 55 & 59 & 34 & 48 & 80 & 93 & 38.21 & 24.16 & 57.00 \\
\hline NE $82^{\text {nd }} \&$ NE Wasco & 46 & 45 & 39 & 43 & 79 & 83 & 38.13 & 25.18 & 57.19 \\
\hline SE $82^{\text {nd }} \&$ SE Mill & 33 & 32 & 34 & 34 & 99 & 104 & 49.69 & 29.28 & 72.21 \\
\hline SE $82^{\text {nd }} \&$ SE Boise & - & 24 & - & 48 & - & 114 & - & 30.55 & 76.53 \\
\hline SE82 ${ }^{\text {nd }} \&$ SE Holgate & - & 97 & - & 123 & - & 315 & - & 28.97 & 72.36 \\
\hline
\end{tabular}

Table 4-3 shows that the minimum average delay per actuation is similar for the five intersections listed above. However, the maximum average delay is higher at three intersections (SE Mill, SE Boise and SE Holgate). One clear limitation of these methods is that the number of pedestrians per actuation is unknown. Thus, delay calculations represent a lower bound. However the actuation trends can be considered as a proxy for crossing demand in the absence of demand data (Day et al., 2011). Long term ranges of average delay per actuation can be estimated and tracked over time to assess whether the average delay is trending upwards or downwards with time. The average delay can be used to calculate pedestrian LOS of each intersection and assess if changes need to be made to improve LOS.

A number of factors impact delay at an intersection. Clearly, longer cycle lengths will, on average, result in longer pedestrian delays. The City of Portland also follows a pedestrian friendly policy of letting the coordination phases rest in walk, if there is no call on the side streets. This policy ensures that pedestrians maximize their allowable walk 
time and minimizes delay for the coordinated phases. However, for the side street phases, delay could potentially increase since the cycle timer has to serve the pedestrian clearance time for the main phases, if there is a call on the side street instead of directly bringing up the yellow phase. The next section(s) will evaluate the impacts of pedestrian friendly strategies such lengthening permissive windows, temporary removal of signals from coordination and shorter cycle lengths on delay.

\subsection{Field Test of Delay Effects of Control Strategies to Favor Pedestrians}

The previous chapters demonstrated the importance of delay in crossing decisions and the use of existing resources for measuring delay. While the primary focus of prior efforts in the development of pedestrian strategies has been safety improvements, this section reports on the findings of field tests of strategies designed to increase efficiency for pedestrians by reducing their delay. The impacts resulting from changes to permissive length and mode of operation were evaluated using before and after measurements of delay and are discussed below.

\subsubsection{Study Locations}

The two locations for in-field tests were chosen on the basis of varying signal configurations (half vs. full signal) and available infrastructure (push buttons, pedestrian actuated phases, communication to the signal controller, 2070 controllers, newer version of signal controller software and pre-programmed internal logic commands to capture actuations and delay). The first location shown in Figure 4-8, NE $33^{\text {rd }}$ Avenue and NE Hancock Street is a half-signal, where the major street approach (NE $33^{\text {rd }}$ Avenue) is 
controlled by a traffic signal and the minor approach (NE Hancock Street) is controlled by a stop sign. The second location, NE Sandy Boulevard and NE $16^{\text {th }}$ Street is fully signalized. At both these intersections, the major street signal phases are coordinated with the adjacent signals along that corridor.

The intersection of NE $33^{\text {rd }}$ Avenue and NE Hancock Street is located in the northeast quadrant of the city of Portland, Oregon. NE $33^{\text {rd }}$ Avenue is the major street at this intersection and has one travel lane in each direction with a left-turn lane. Hancock Street is the minor street at this intersection.

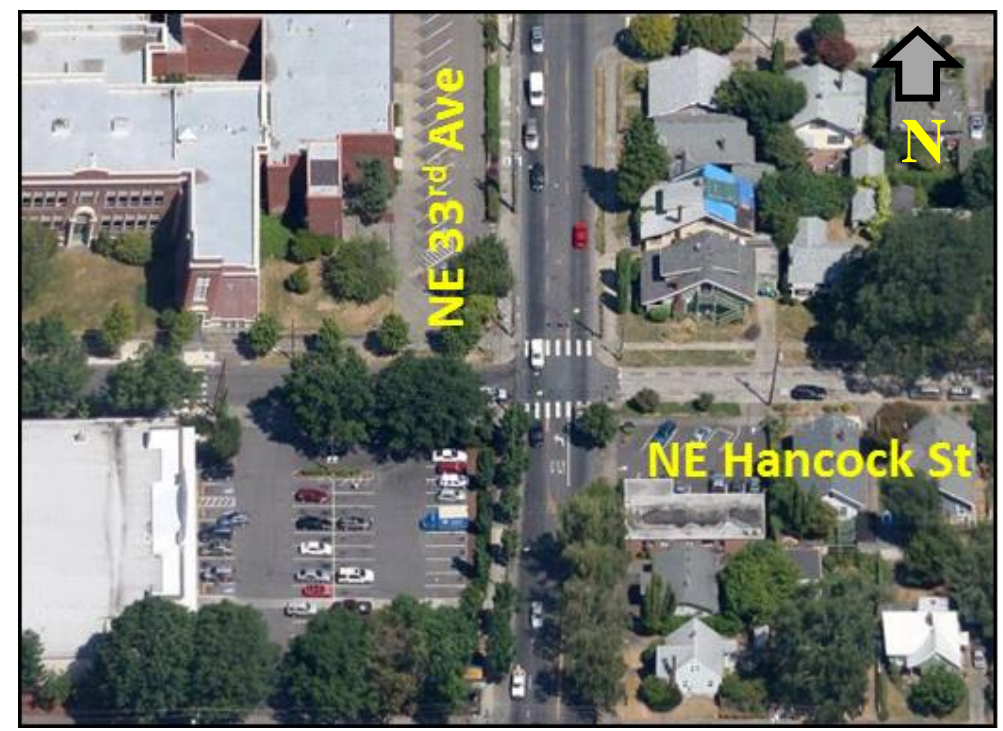

Figure 4-8 NE 33rd Avenue and NE Hancock Street

Two signal phases are in operation at this intersection as shown in Figure 4-9. Phase 2 is associated with the major street and Phase 4 is associated with the pedestrian signals on the minor approach. The minimum and maximum green times for phase 2 are 20 and 50 sec respectively. Prior to this study, this intersection was operating in a coordinated mode with fixed time of day plans. Four coordination plans were in operation at this 
intersection. Plans 1-3 have a common cycle length of $90 \mathrm{sec}$ with the only differences being in the offset. Plan 4, which operates during the off-peak periods, has a cycle length of $70 \mathrm{sec}$. Figure 4-10 shows the time of day schedule for this intersection.
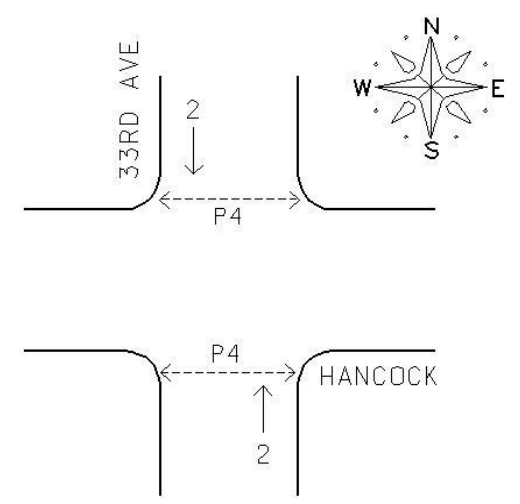

PHASE DIAGRAM

Figure 4-9 Phase Diagram for NE 33rd Avenue and NE Hancock Street

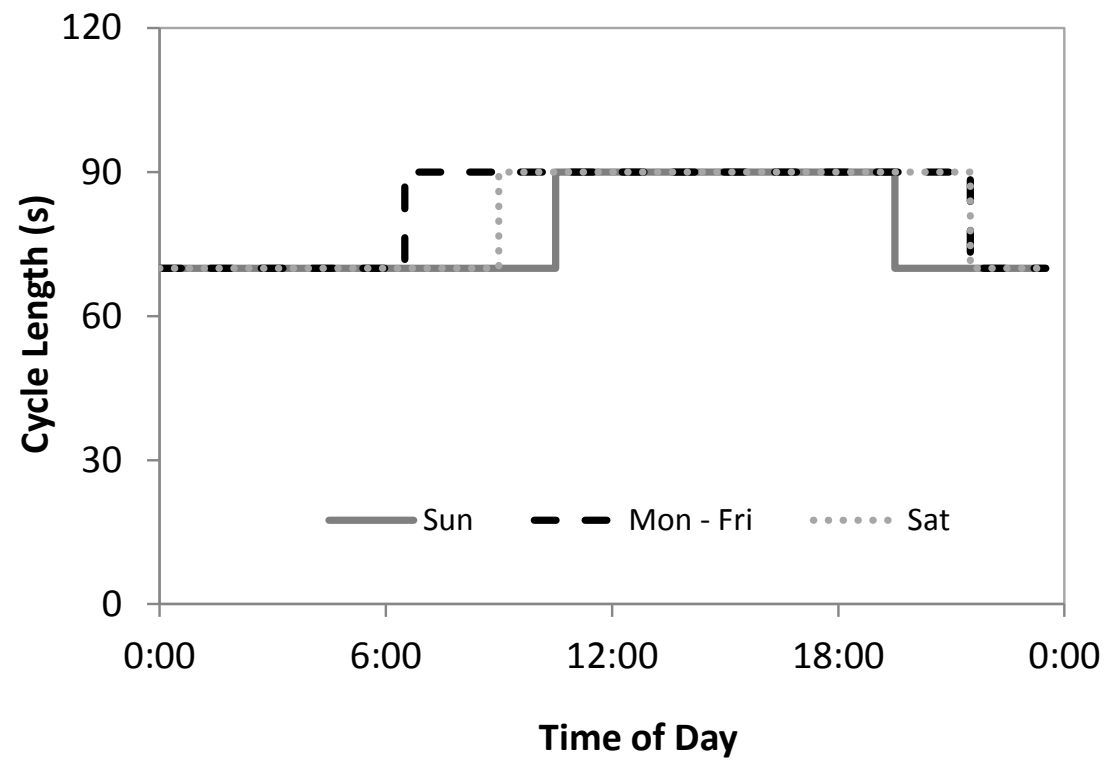

Figure 4-10 Coordination Schedule and Time of Day Plans

Cycle lengths varied between 70 and $90 \mathrm{sec}$ at this intersection. Timing plans with longer cycle lengths are used during the day to accommodate greater vehicle demand and shorter 
cycle lengths are used during night and early morning hours to be more responsive to pedestrians. Longer cycle lengths may be beneficial for vehicles on the major approach due to the longer green times for the vehicles; they are disadvantageous for pedestrians due to longer delay.

During coordinated operation the delay experienced by a pedestrian depends on when the call is received during the cycle by the signal controller. The delay is shorter if the call is received within the permissive period because the controller allows the call to be serviced. According to the Signal Timing Manual, permissive length is defined as a period of time after the yield point where a call on a non-coordinated phase can be serviced without delaying the start of the coordinated phase (Koonce et al., 2008). For increased clarification, pedestrian calls are defined as early or late depending on when in the cycle they are received by the signal controller. A call is designated as an early call if it is received prior to the start of the permissive period or the yield point. If the call is received during the permissive period, it is defined as a late call. Figure 4-11 shows circle diagrams illustrating how the signal controller responds to an early and late pedestrian call for the base condition (permissive $=19 \mathrm{sec}$ ). If a pedestrian "early" call is received during the phase 2 green interval - after cycle time 19 and before the end of the cycle (70 or $90 \mathrm{sec}$ depending on the cycle length) - the pedestrian is served during the following cycle at cycle time 5.6 after the clearance (YAR) for phase 2 has been served. If a call comes in between 0 and $19 \mathrm{sec}$ in the cycle timer, a "late" call gets served during the same cycle and results in the shortest delay (equal to the clearance time for phase 2). The increased permissive tested in this research increases the amount of time that a "late" 
pedestrian call can be served during the current cycle. In summary, early calls will have longer delay and late calls within the permissive period have the shortest delay.

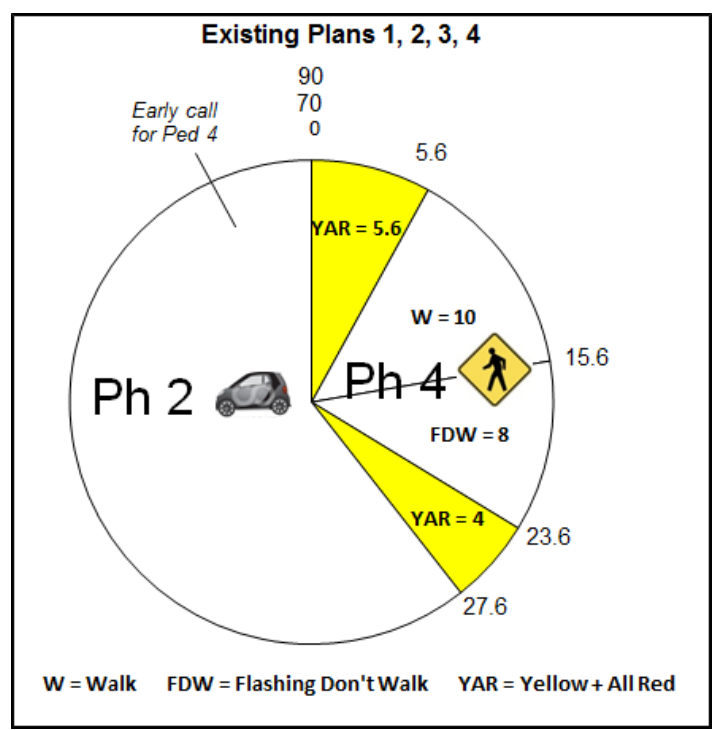

(a)

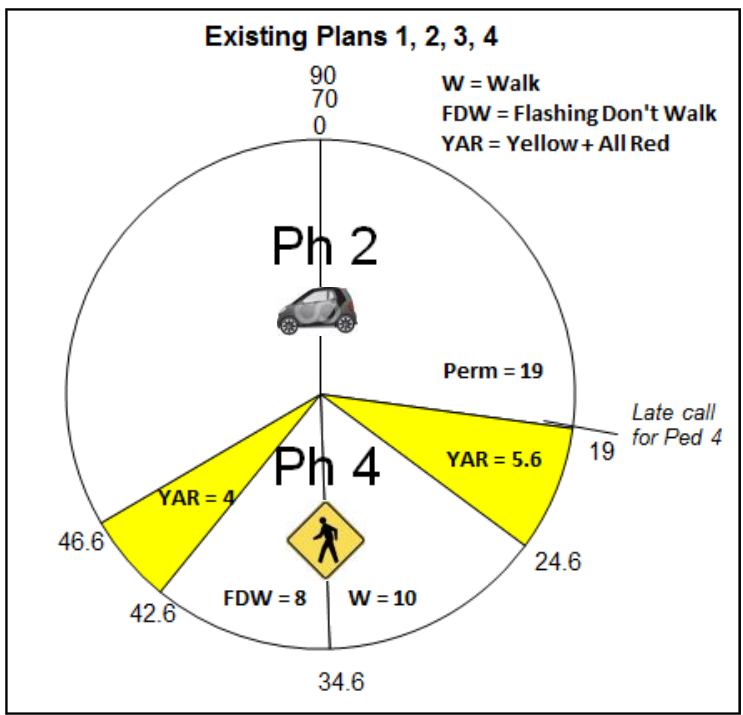

(b)

Figure 4-11 Early and Late Pedestrian Calls

The strategy tested at this intersection was to increase the permissive length, while remaining in coordination. The results are discussed in the following section.

The second study intersection is also located in northeast Portland, Oregon. NE Sandy Boulevard is the major street and is a 4-lane arterial with two travel lanes in each direction. The minor street at this intersection is NE $16^{\text {th }}$ Ave which is oriented in the north-south direction. Businesses occupy all four corners of this intersection. Figure 4-12 shows the aerial view of this intersection.

Four signal phases are in operation at this intersection as shown in Figure 4-13. Phases 2 and 6 are associated with the eastbound and westbound movements on NE Sandy Blvd, and phases 4 and 8 are associated with the northbound and southbound 
movements on NE $16^{\text {th }}$ Ave. Phases 2 and 6 are on vehicle and pedestrian recall. Therefore, pedestrian actuations and delay data were collected only for phase 4, which corresponds to the east leg crosswalk crossing NE Sandy Blvd. In the normal mode of operation, this intersection was coordinated all the time. In the absence of vehicle or pedestrian calls on phases 4 and 8 , the signal controller allots the unused time in the cycle to phases 2 and 6 , and also rests in walk for pedestrian phases 2 and 6 . While this strategy is good for pedestrians crossing NE $16^{\text {th }}$ Ave, it induces extra delay for pedestrians requesting service for crossing NE Sandy Blvd, due to the necessity to provide clearance time (Flashing Don't Walk) for the pedestrian phases prior to switching to phases 4 and 8.

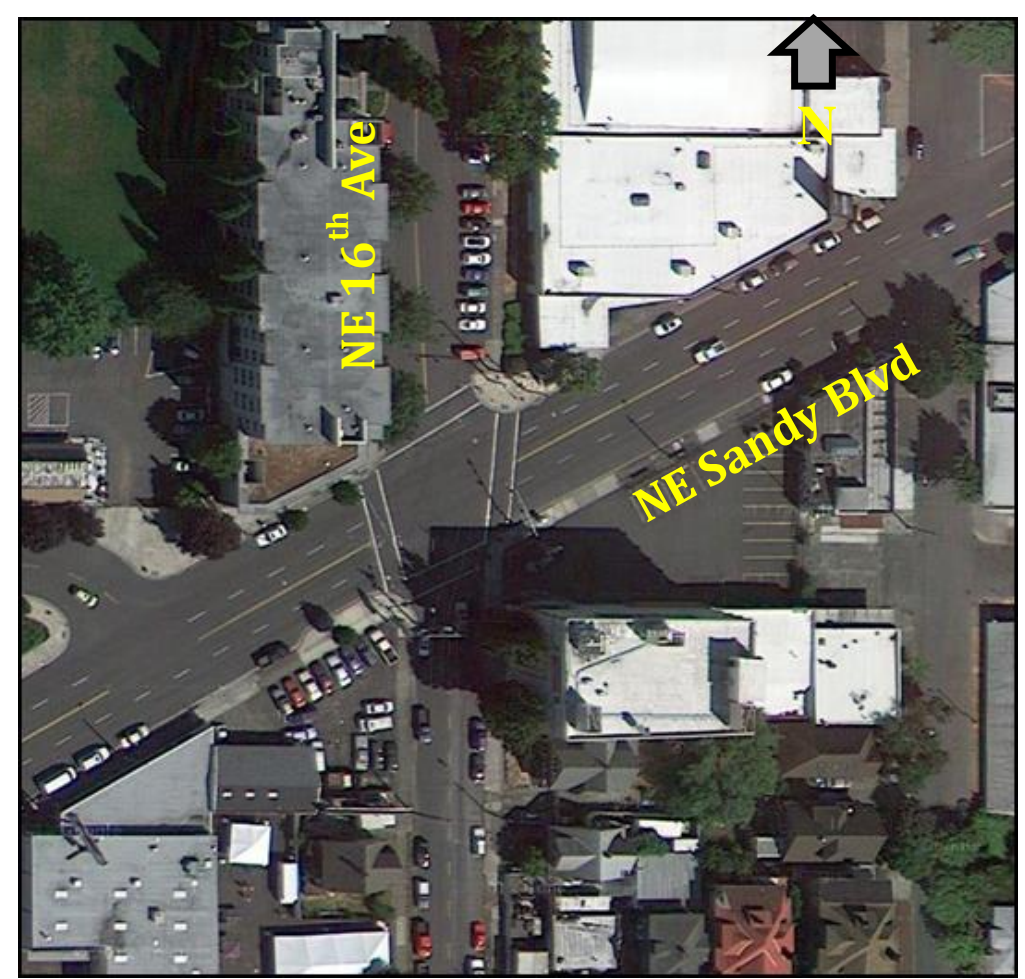

Figure 4-12 NE Sandy Boulevard and NE 16th Avenue 


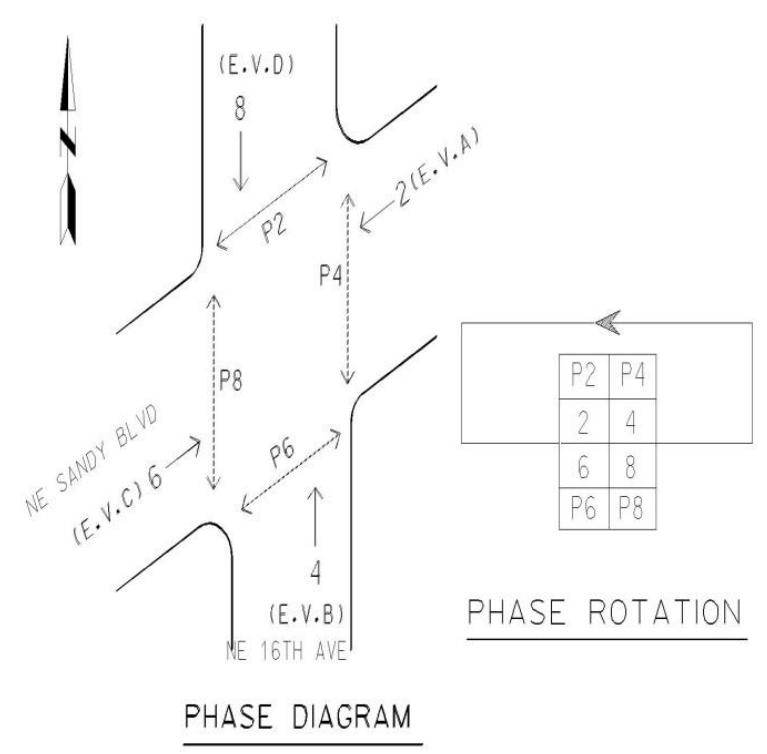

Figure 4-13 Phase Diagram for NE Sandy Boulevard and NE 16th Avenue

Four coordination plans are in operation at this intersection, all having a common cycle length of $70 \mathrm{sec}$ with the only difference between the plans being in the offsets. The strategy followed at this intersection to reduce pedestrian delay was to selectively remove the signal from coordination during the off-peak traffic periods. Allowing a signal to operate in free mode can result in variable cycle lengths, and more responsive pedestrian operations. The limitation with the current HCM pedestrian analysis is that there is no procedure to estimate the benefits of free operation. The resulting outcomes and findings are discussed in the next section.

\subsubsection{Analysis and Results}

Two methods previously discussed in section 4.3 were used to measure and automatically record pedestrian actuations and delay at two chosen locations. Actuation refers to the count of push button activations and delay is measured as the time difference between push button actuation and walk phase being served. Internal logic commands using 
Northwest Signal (NWS) Voyage software were programmed into Type 2070 controllers at the two locations to collect the required data.

At NE $33^{\text {rd }}$ Ave and NE Hancock St, the transit priority logging ability of the controller was used to collect actuations and measure delay. As outlined earlier, the controller at this intersection was coordinated based on time of day plans. In order to quantify the change in average delay per actuation based on changes to the permissive length and mode of operation, the transit priority logs that recorded pedestrian actuations and delay were collected prior to and after each change was made. Four scenarios were tested at this intersection. Data for the base (existing) condition was collected during June 19 - June 24, 2012. The permissive length was then increased from $19 \mathrm{sec}$ to $28 \mathrm{sec}$ and data pertaining to this condition (Scenario 1) was collected during June 26 - July 1, 2012. The permissive length was further increased to $35 \mathrm{sec}$ (Scenario 2) and data were collected during the period July 3 - July 8, 2012. The third scenario (Scenario 3) involved selectively increasing the permissive length from $19 \mathrm{sec}$ to $35 \mathrm{sec}$ only during the offpeak traffic periods. Data for this scenario was collected during July 10 - July 15, 2012. The increases in permissive length were manually determined. Finally, the controller was set free for all time periods (Scenario 4) and data were collected during July 17 - July 22, 2012. The base permissive length $(19 \mathrm{sec})$ was obtained from the controller and other values tested in this research $(28 \mathrm{sec}, 35 \mathrm{sec})$ were manually determined.

A pedestrian call is classified as an early or late call as outlined previously in this section. For Scenarios 2 and 3, if the pedestrian call comes in early, then the circle diagram depicted in Figure 4-11 (a) applies and the controller operates as before. The 
circle diagrams for a late pedestrian call for permissive lengths of $28 \mathrm{sec}$ and $35 \mathrm{sec}$ are shown in Figure 4-14 (a) and (b), respectively. The benefit of increasing the permissive length is accrued primarily when a late pedestrian call is received. Increasing the permissive length to $28 \mathrm{sec}$ and $35 \mathrm{sec}$ ensures that a call received between cycle time 19 and 28 , or cycle time 19 and 35 , respectively, will get served in the same cycle, thereby decreasing pedestrian delay. However a late pedestrian call in one cycle may impact the delay for the next pedestrian call depending on the magnitude of the minimum green time and cycle length for phase 2. At this location, it can occur during time periods when the cycle length is $70 \mathrm{sec}$, a late pedestrian call has been served (permissive $=28$ or 35) and there is early pedestrian call for service after the late call. If there is not enough time remaining in the cycle for minimum green for phase $2(20 \mathrm{sec})$ to be served, phase 2 will finish timing beyond the yield point and then the early pedestrian call is served. Delays resulting from back to back late and early pedestrian calls when cycle length is $90 \mathrm{sec}$ are not impacted. 


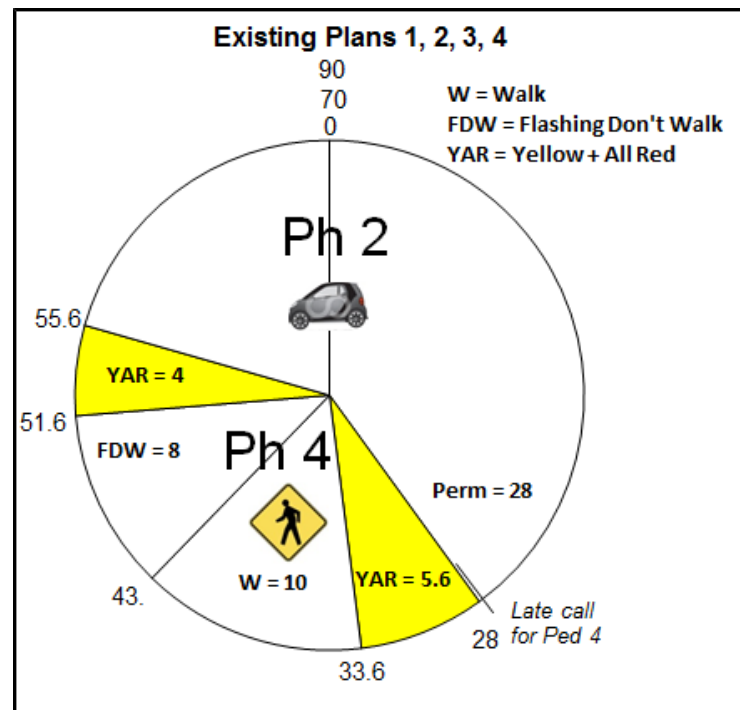

(a)

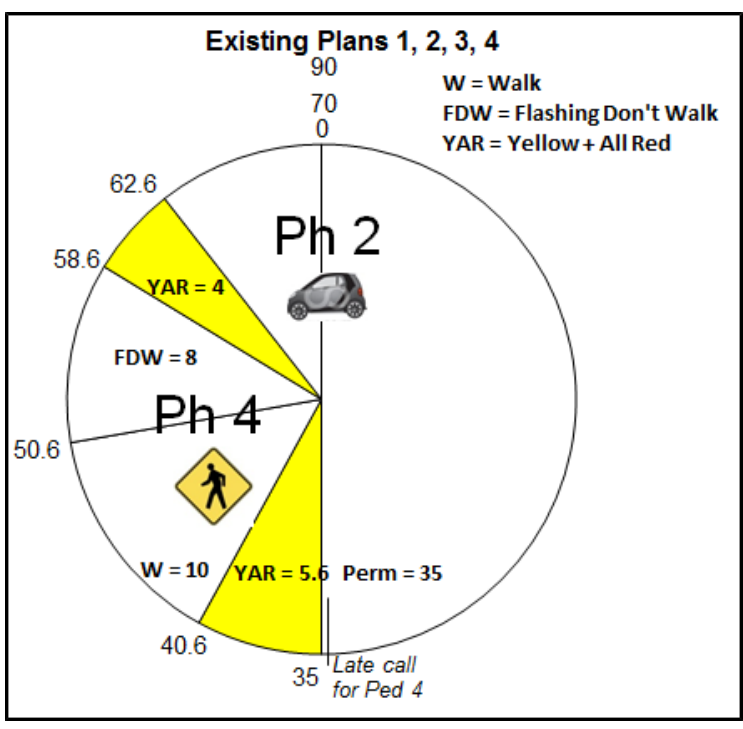

(b)

Figure 4-14 Late Pedestrian Calls for Permissive Length of $28 \mathrm{~s}$ and $35 \mathrm{~s}$

Since two cycle lengths were operational at this intersection, the average delays obtained for the base conditions and well as the different scenarios were tested separately for each cycle length so as to eliminate the effect of cycle lengths, as it is well understood that longer cycle lengths lead to greater delays. Figure 4-15 (a)-(d) show plots of measured pedestrian delay as a function of the actuation time (with respect to the local cycle timer) for the base condition and Scenarios 1-3 for cycle length of $90 \mathrm{sec}$. The above figures show the benefit of increasing the permissive length via reductions in pedestrian delay. Figure 4-15 (a) shows that any pedestrian calls received between cycle time 0 and $19 \mathrm{sec}$ have delay equal to the clearance time for phase 2 of $5.6 \mathrm{sec}$. Calls received after $19 \mathrm{sec}$ have higher magnitudes of delay as seen in the plot, and this delay keeps decreasing as the calls are received later in the cycle. Figure 4-15 (b) - (d) show that pedestrian delay for calls received between cycle time 19 through 28,19 through 35 , and a combination of the two, remains at $5.6 \mathrm{sec}$ due to the increase in the permissive length. The outliers seen 
in the plots are a result of the pedestrian calls received during transition periods when the controller is switching between coordination plans. Figure 4-16 (a) and (b) show the distributions of delay for the base condition as well as Scenarios 1-3 for both cycle lengths.

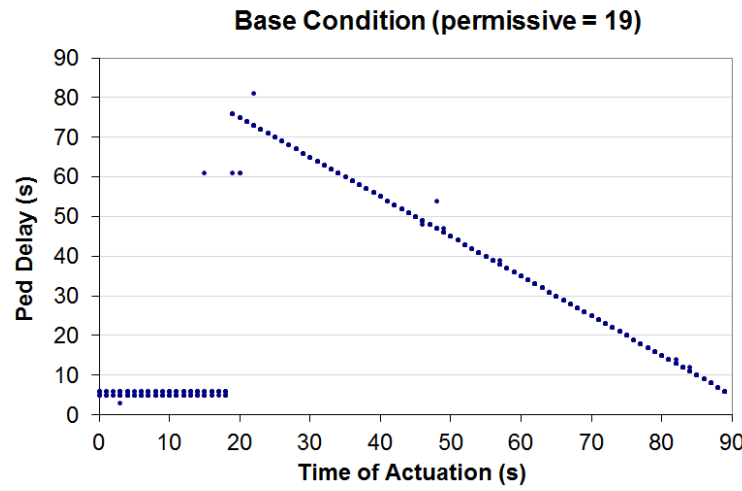

(a)

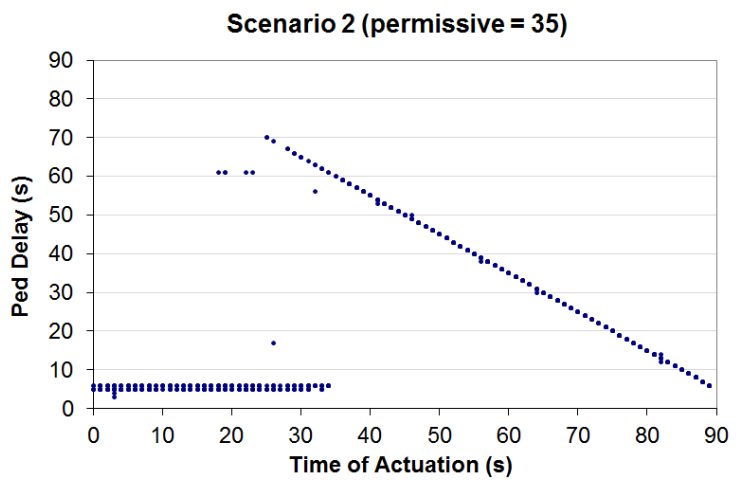

(c)

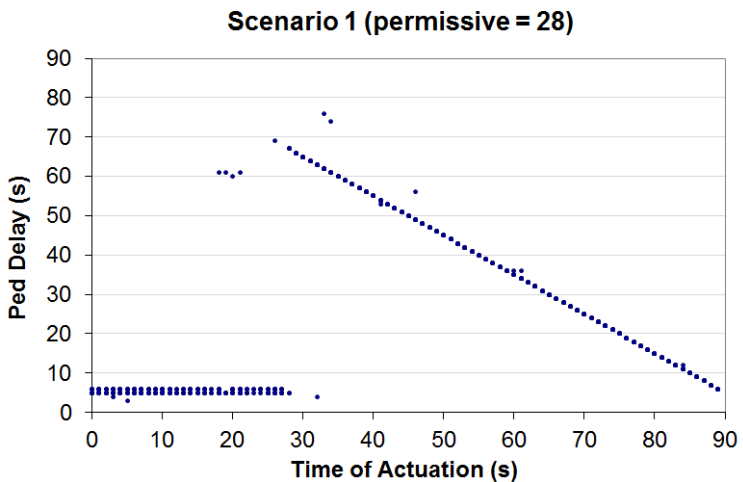

(b)

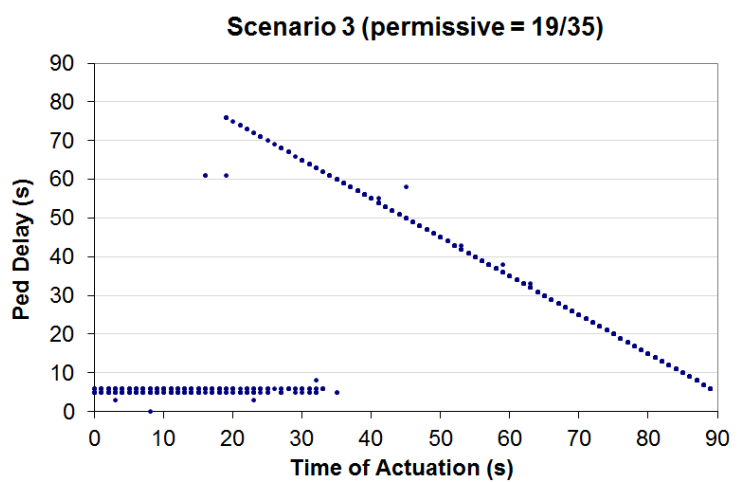

(d)

Figure 4-15 Pedestrian Delay vs. Time of Actuation 


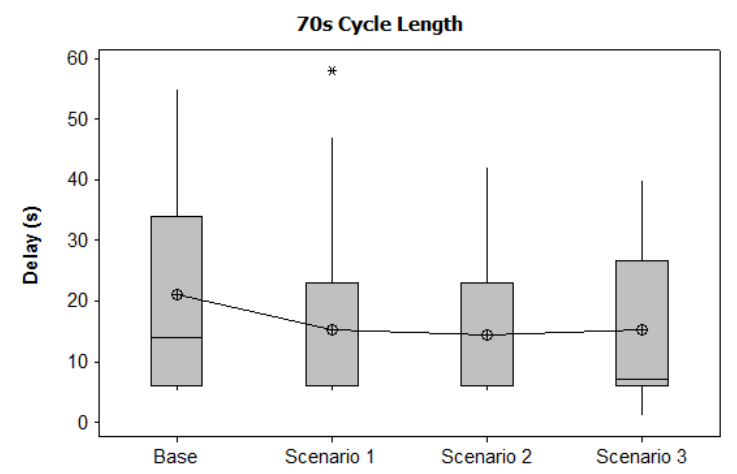

(a)

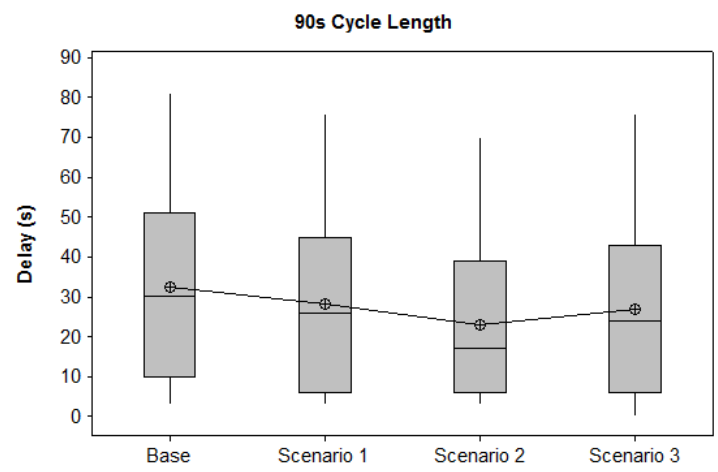

(b)

Figure 4-16 Box Plots of Pedestrian Delay

Descriptive statistics including average delays for the base condition and each of the scenarios for the two cycle lengths are shown in Table 4-4. Also included in Table 4-4 are statistics for Scenario 4, which represents the signal operating in free mode with no set cycle length. Note that the HCM estimated values for pedestrian delay are $22.4 \mathrm{sec}$ and $32.08 \mathrm{sec}$ for cycle lengths of 70 and $90 \mathrm{sec}$, respectively, and it does not change with either permissive length or mode of operation changes.

As seen in Table 4-4, the average delays for Scenarios 1, 2 and 3 have decreased compared to the base values for both cycle lengths. The average delay for Scenario 4 is the lowest, indicating that when a signal operates in a free mode it is beneficial for pedestrians. However, in order to test for significant differences an ANOVA was performed. The Analysis of Variance or ANOVA is used to test the hypotheses that the means among two or more groups are equal. The hypotheses for this test are stated below.

$\mathrm{H}_{0}$ :There is no difference in population means for the different groups $\mathrm{H}_{1}$ : ot all popul ation means are equal 
Table 4-4 Descriptive Statistics for Different Scenarios

\begin{tabular}{|c|c|c|c|c|c|c|c|c|c|}
\hline \multirow[b]{2}{*}{ Statistic } & \multicolumn{4}{|c|}{ Cycle Length (70s) } & \multicolumn{4}{|c|}{ Cycle Length (90s) } & \multirow{2}{*}{$\begin{array}{c}\text { Free } \\
\text { Scenario } \\
4\end{array}$} \\
\hline & Base & $\underset{1}{\text { Scenario }}$ & $\begin{array}{c}\text { Scenario } \\
2\end{array}$ & $\begin{array}{c}\text { Scenario } \\
3\end{array}$ & Base & $\begin{array}{c}\text { Scenario } \\
1\end{array}$ & $\begin{array}{c}\text { Scenario } \\
2\end{array}$ & $\begin{array}{c}\text { Scenario } \\
3\end{array}$ & \\
\hline Mean & 21.03 & 15.22 & 14.25 & 15.11 & 32.38 & 28.29 & 23.09 & 26.99 & 11.46 \\
\hline Median & 14 & 6 & 6 & 7 & 30 & 26 & 17 & 24 & 6 \\
\hline Mode & 5 & 6 & 6 & 6 & 6 & 6 & 6 & 6 & 6 \\
\hline Std. dev & 16.59 & 13.02 & 12.22 & 11.91 & 22.48 & 20.29 & 18.73 & 20.11 & 12.80 \\
\hline Range & 50 & 53 & 37 & 39 & 78 & 73 & 67 & 76 & 59 \\
\hline Min & 5 & 5 & 5 & 1 & 3 & 3 & 3 & 0 & 5 \\
\hline Max & 55 & 58 & 42 & 40 & 81 & 76 & 70 & 76 & 64 \\
\hline Count & 85 & 108 & 123 & 114 & 1057 & 1036 & 1002 & 1193 & 1221 \\
\hline
\end{tabular}

The statistical software Minitab was used to perform the test for each value of cycle length. For the 70 second cycle length, the p-value for the ANOVA test was obtained as 0.002. Since, the $\mathrm{p}$-value is less than $\alpha=0.05$, the null hypotheses is rejected. For the 90 sec cycle length, the p-value is obtained as 0.000 . Therefore, in this case also, the null hypothesis is rejected. Tukey's post-hoc test was conducted to see which population means showed a significant difference. Table 4-5 shows the results of Tukey's test.

Table 4-5 Post hoc Tests for Different Scenarios

\begin{tabular}{l|rrrr}
\hline Cycle Length & \multicolumn{1}{|c}{ Source } & \multicolumn{1}{c}{ Count } & Mean & Grouping \\
\hline \multirow{4}{*}{70} & Base & 85 & 21.04 & $\mathrm{~A}$ \\
& Scenario 1 & 108 & 15.22 & $\mathrm{~B}$ \\
& Scenario 2 & 123 & 14.25 & $\mathrm{~B}$ \\
& Scenario 3 & 114 & 15.11 & $\mathrm{~B}$ \\
\hline \multirow{4}{*}{90} & Base & 1057 & 32.38 & $\mathrm{~A}$ \\
& Scenario 1 & 1036 & 28.29 & $\mathrm{~B}$ \\
& Scenario 2 & 1002 & 23.09 & $\mathrm{C}$ \\
& Scenario 3 & 1193 & 26.99 & $\mathrm{~B}$ \\
\hline
\end{tabular}

From Table 4-5, it is observed that for the $70 \mathrm{sec}$ cycle length there was a significant difference between the average delays for the base case and Scenarios 1,2 and 3. However, no significant difference was found between Scenarios 1, 2 and 3 indicating that while the change in permissive length reduced average delay, further increase of the 
permissive length and using different values of permissive lengths during the peak and off-peak periods did not produce a significant reduction in average delay. For the $90 \mathrm{sec}$ cycle length a significant difference was found between the base case and all three scenarios. In addition, Scenarios 1 and 3 were not significantly different from each other, indicating that using a permissive length of 19 or $28 \mathrm{sec}$ in the peak, and $35 \mathrm{sec}$ in the offpeak was not statistically significant. However, Scenario 2 was statistically significant indicating that using a permissive length of $35 \mathrm{sec}$ produced a significant reduction in average delay per actuation.

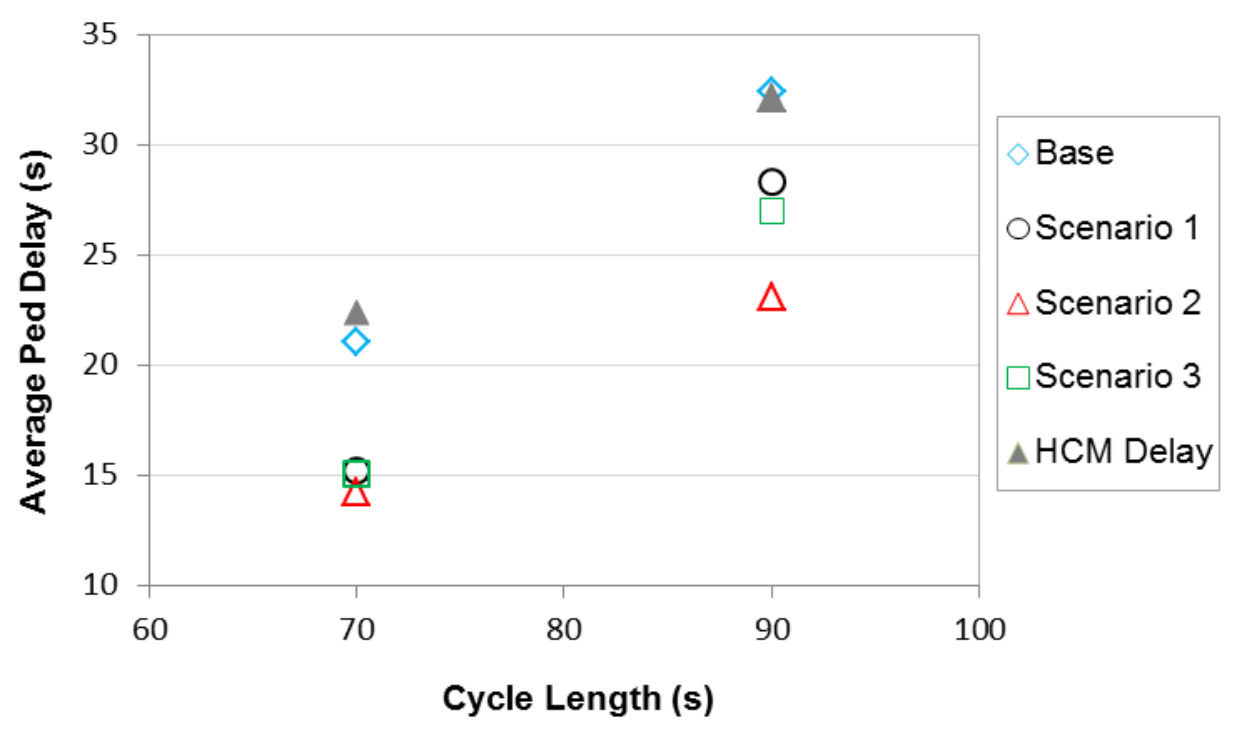

Figure 4-17 Pedestrian Delay vs. Cycle Length

Figure 4-17 shows the average delay obtained for different scenarios as well as the HCM estimated delay as a function of cycle length. While in coordination, it is apparent that pedestrians in Scenario 2 (largest permissive length increase) experienced the shortest delay irrespective of cycle lengths. 
NE Sandy Blvd. and NE $16^{\text {th }}$ Avenue was coordinated at all times in its base (existing) condition with a common cycle length of $70 \mathrm{sec}$ across various coordination plans. Since this signal is located on a major arterial with significant vehicle traffic, the strategy adopted at this intersection in order to achieve a reduction in pedestrian delay, was to remove the signal out of coordination only during off-peak hours, while keeping it in coordination during the AM (6:30 - 9:00) and PM (15:30 - 18:45) peak periods on weekdays (Monday - Friday).

The data available for pre and post analysis were in the form of binned delay from the volume logs. Data for the existing condition were collected during June 26 - July 2, 2012. Data for the test scenario was collected during July 10 - July 16 , 2012. Since the volume bin method provides less detailed data, it was not possible to perform similar analysis as with the data for NE $33^{\text {rd }}$ Ave and NE Hancock St. Instead the distribution of binned delay was studied for the existing and test scenarios. Figure 4-18 shows the distribution of binned delay.

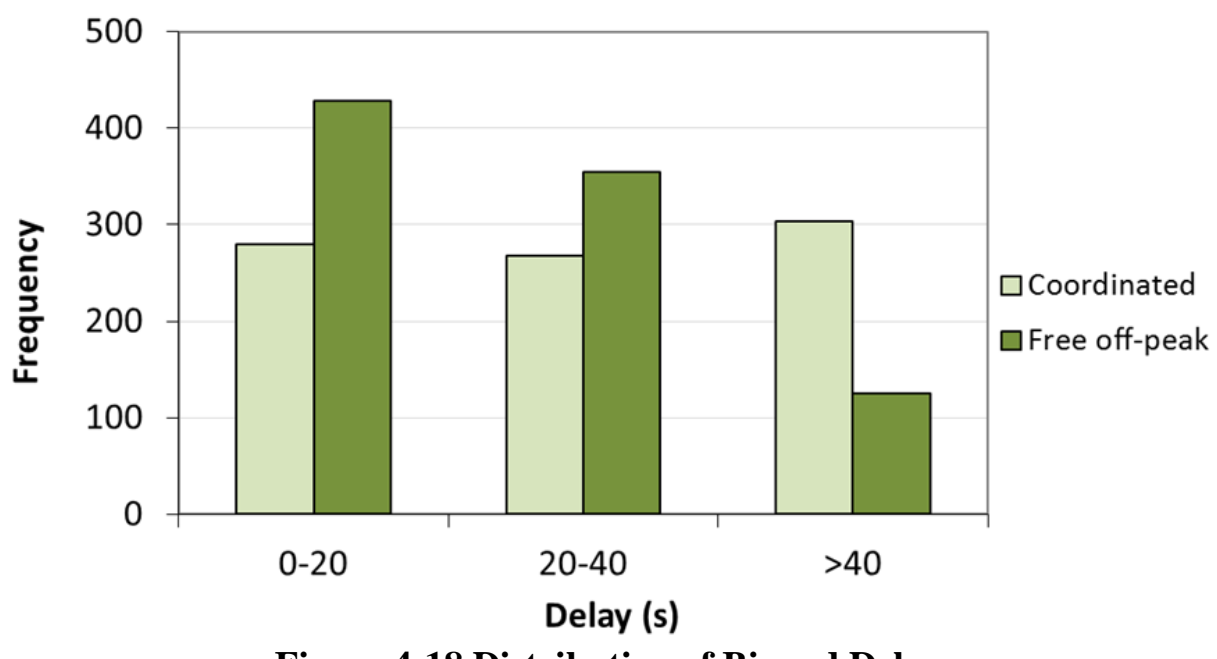

Figure 4-18 Distribution of Binned Delay 
A total of 852 actuations were observed in the one week period when the signal was operating in coordinated mode, and 908 actuations were recorded during free off-peak operation. From Figure 4-18, it is observed that during coordinated operation the bin with delays greater than $40 \mathrm{sec}$ has the highest frequency, whereas during the free off-peak operation, the trend is reversed indicating the distribution of delay has shifted. To test if the shift was significant, a chi-square two sample test was performed. The null and alternate hypotheses for the chi square test are stated below.

$\mathrm{H}_{0}$ : The two samples come from a common distribution $\mathrm{H}_{1}$ :The two samples do not come from a common distribution

The p-value is obtained as 0.000 , which is less than the 0.05 level of significance. Therefore the null hypothesis is rejected and there is evidence to indicate that distributions are significantly different. Further analysis of the actuations with the highest delay indicates that these occurred during the coordinated AM and PM peak periods. The results obtained at this location indicate that operating the signal in free mode during the off-peak periods is beneficial to pedestrians and reduces their delay.

\subsection{Summary}

Delay is an important performance metric from an efficiency perspective and is often used to characterize the performance of the intersection via level of service calculations. Although some cities and jurisdictions have a stated policy of "pedestrians first", signal 
timing policies have traditionally favored vehicles over pedestrians, leading to large delays for pedestrians. Prior research has established the link between large delays and risky behaviors leading to signal non-compliance.

This chapter outlined two methods to measure pedestrian delay using existing Type 2070 signal controllers and pushbutton actuations. These methods were developed using existing resources and hence are cost-effective and easily deployable in the field. The actuation and delay data obtained from these methods enables the development of pedestrian performance measures and allows an engineer to characterize pedestrian service at intersections. In field tests of alternate pedestrian strategies: permissive length increase and change in mode of operation were conducted and the resulting impacts on pedestrian delay were analyzed at two locations. Increasing permissive length resulted in significantly reduced delay for pedestrians. Cycle length impacts on pedestrian delay were also observed, with larger cycle lengths leading to higher delay for pedestrians. The mode of operation also had an impact on pedestrian delay, operating the signal in free or uncoordinated mode led to statistically significant lower pedestrian delays. The HCM equation for pedestrian delay estimation was confirmed to be deficient since it does not incorporate the impacts of permissive length and/or mode of operation.

While field deployments of these strategies allowed the study of impacts on pedestrians through the measurement of pedestrian delay, impacts on other modes could not be studied as the related performance metrics could not be obtained from the signal controller. Therefore, micro-simulation techniques were utilized to study the effects of alternate pedestrian strategies on all modes and are presented in the next chapter. 


\section{EXPLORING PEDESTRIAN TIMING STRATEGIES THROUGH SIMULATION MODELING}

This chapter describes the micro-simulation modeling framework in VISSIM and analyzes the impacts on all modes as a result of change in mode of signal controller operation. Vehicle volumes are varied in three ranges based on volume-capacity ratios $(\mathrm{V} / \mathrm{C})$ of less than $0.3,0.3-0.7$ and greater than 0.7 . Pedestrian volumes are also varied in three ranges based on actuation frequency and frequency of pedestrian calls in the design time period, which is one hour. Forty two simulation models are run to determine the feasibility ranges for coordinated or free operation and control strategies to benefit pedestrians are discussed.

\subsection{Background}

A number of tools have been developed for analyzing traffic. Traffic analysis tools are typically grouped into analytical and simulation models. Analytical models use mathematical formulations to determine traffic states (capacity, density, speed, delay and queuing) on facilities (Akcelik, 2007). These tools are specifically suited for analyzing small scale facilities. Simulation models are often used to model traffic flows in a network. These models can be multi-modal in nature and are used to model the interactions between different modes on a transportation network. These tools are useful in evaluating design alternatives and for decision making purposes. There are three categories of simulation models - macroscopic, mesoscopic and microscopic models. In macroscopic models, the simulation takes place on a section basis, without explicitly 
considering individual vehicles. Some well-known examples of macroscopic simulation models are PASSER, SYNCHRO, TRANSYT and TRANSYT7F. Mesoscopic models are a blend of Macroscopic and Microscopic models. Micro simulation models model the movement of individual vehicles in the traffic stream based on car-following and lanechanging models. The most popular among these are PARAMICS, AIMSUN, VISSIM, SIMTRAFFIC and CORSIM.

Micro simulation models are being increasingly used as an analysis tool worldwide. The advantages of micro simulation models are their ability to model system wide impacts of alternatives and various geometric configurations. While these models can provide detailed statistics, there are a few issues worth noting. These models often require large amounts of data and the accuracy of data inputs into the simulation model affects the precision of results. These models also need to be properly calibrated and validated to yield accurate results. Some degree of user skill is also required to build a representative model.

In this research, VISSIM micro simulation software is used to model the interactions between vehicles, pedestrians and bicycles on an urban street network to evaluate the impacts of changing the signal controller mode of operation from coordinated to free, and to assess what traffic regimes are best suited for each mode of operation. The following sections describe the steps taken in model development, calibration and validation. 


\subsection{Model Development}

The data required for the simulation and validation were gathered via field observations either manually or extracted from video. The basic steps followed during the modeling process are:

1. Site selection

2. Data collection

3. Network development

4. Calibration

5. Validation

Each of these steps is discussed in the following sections.

\subsubsection{Site Selection}

Site selection was based on certain requirements such as presence of traffic signals along the selected route, coordinated operations, presence of heavy pedestrian activity along the corridor (pedestrians and bicycles). NE Multnomah Street in Portland, Oregon was chosen as the study corridor for this research. Three signalized intersections were chosen for analysis: NE Multnomah Street and NE $11^{\text {th }}$ Avenue, NE Multnomah Street and NE $13^{\text {th }}$ Avenue, and NE Multnomah Street and NE $15^{\text {th }}$ Avenue. These are shown in Figure 5-1. These intersections are about $500 \mathrm{ft}$ apart with eleven pedestrian crosswalks. This corridor is a part of the Lloyd District, which is a commercial neighborhood in Northeast Portland, OR. The Lloyd District is bounded by N/NE Broadway to the north, NE $16^{\text {th }}$ Avenue to the east, I-84 to the south and Willamette River to the west. This area includes 
destinations such as Lloyd Center Mall, a movie theater, various office buildings and restaurants.

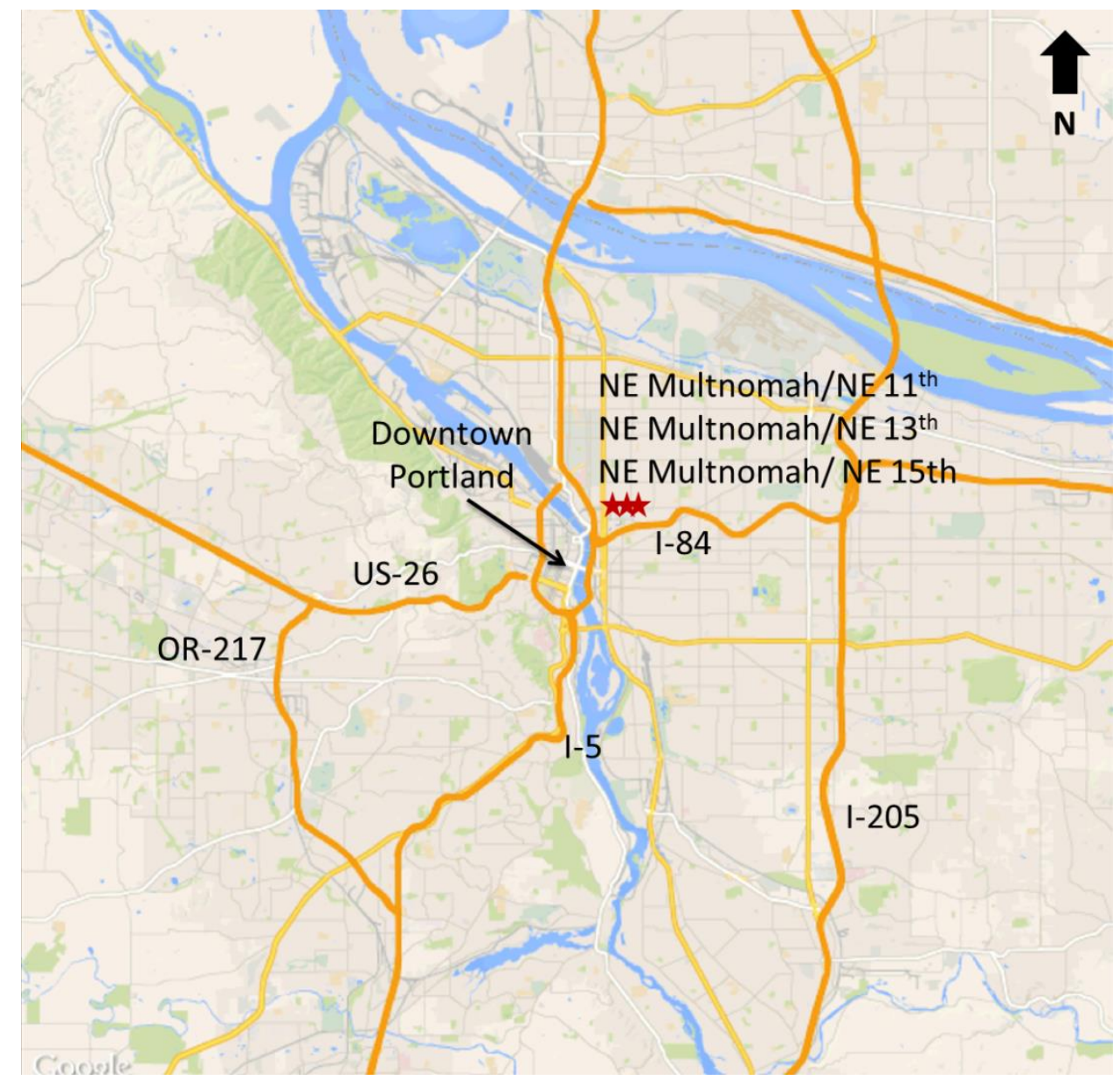

Figure 5-1 Intersections Chosen for Simulation

Prior to 2012, Multnomah Street had 5 lane cross-section, with two travel lanes in the east-west direction along with a center turn lane as shown in Figure 5-2. In late 2012, the corridor was reconfigured to a 3 lane cross-section, with one travel lane in each direction, with additional turn lanes at intersections. A protected cycle track was added in the east 
and westbound direction for bicyclists. Figure 5-3 shows the new cross-section of NE Multnomah Street.

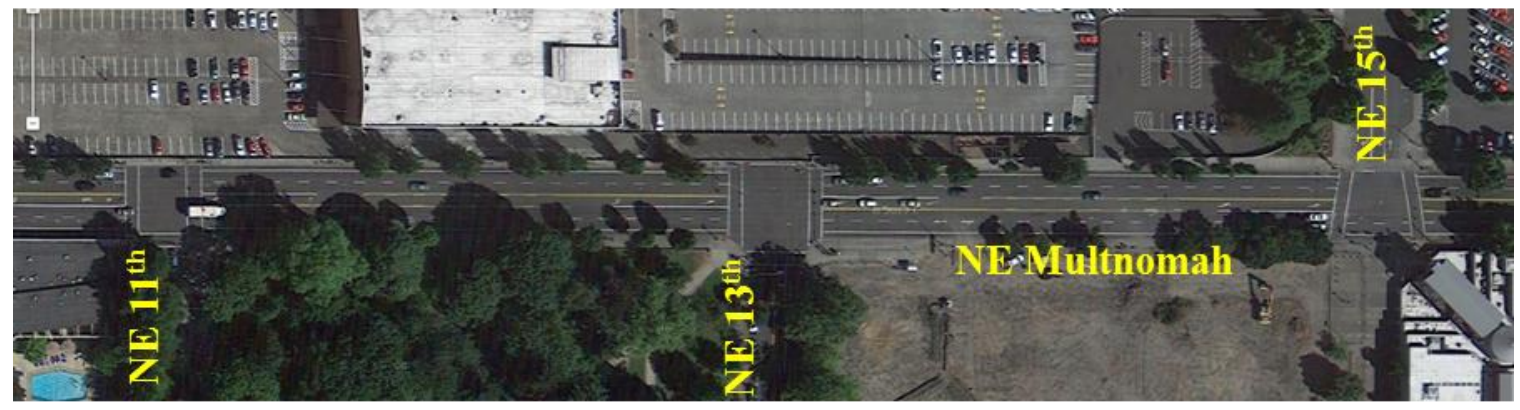

Figure 5-2 NE Multnomah Street Old Cross-section

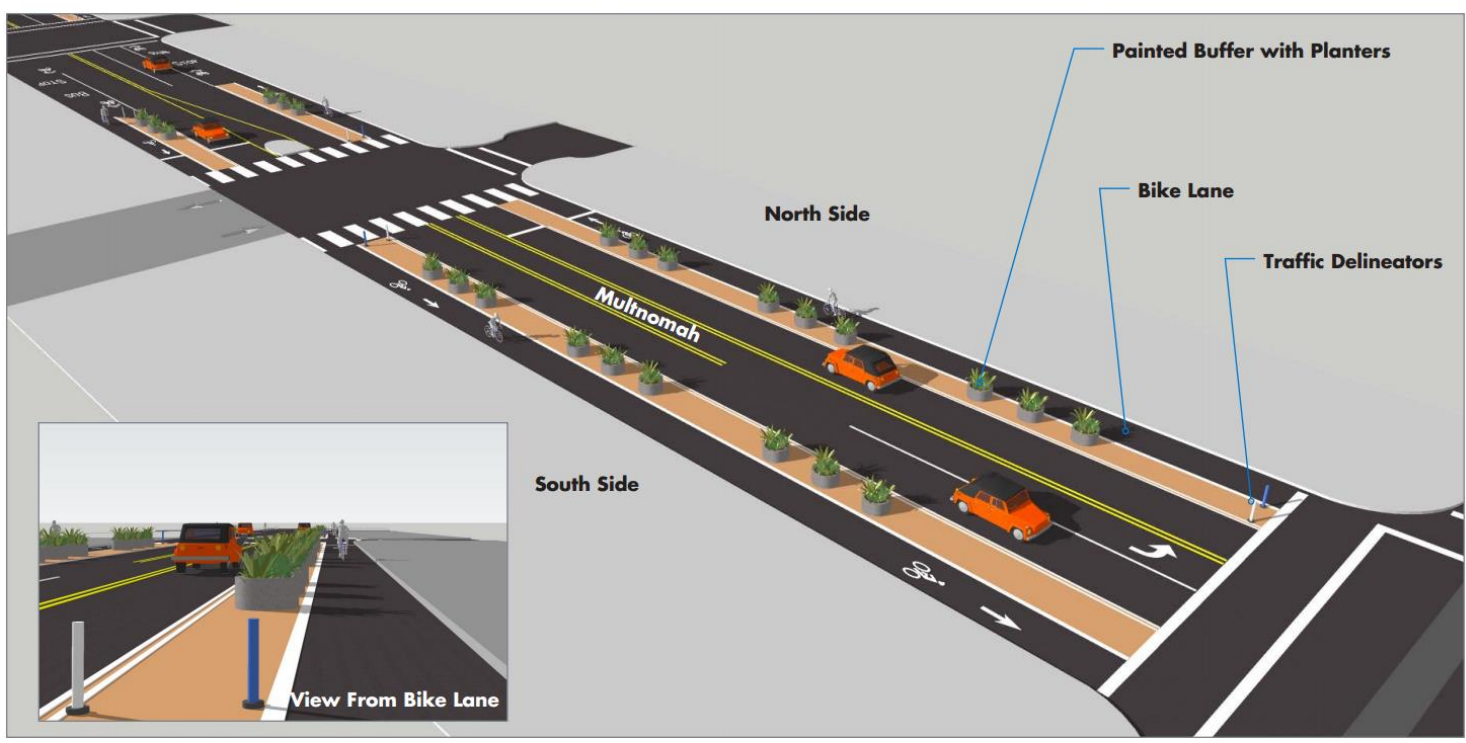

Figure 5-3 NE Multnomah Street New Cross-section with Cycle Track (Source: Portland Bureau of Transportation)

\subsubsection{Data Collection}

Vehicle, bicycle and pedestrian volumes were required as a necessary input for the simulation model. Vehicle and bicycle volumes were collected using pneumatic tube counters at five locations for 24 hours as shown in Figure 5-4 on Thursday, October 3, 2013. At four locations (Multnomah east and westbound, $11^{\text {th }}$ and $13^{\text {th }}$ northbound) 
pneumatic tubes by MetroCount ${ }^{\circledR}$ were used and at the fifth location (15 ${ }^{\text {th }}$ southbound), Pico tube counter was used. The Metrocount ${ }^{\circledR}$ tubes were capable of counting bicycles and vehicles whereas the Pico counters could only count vehicles.

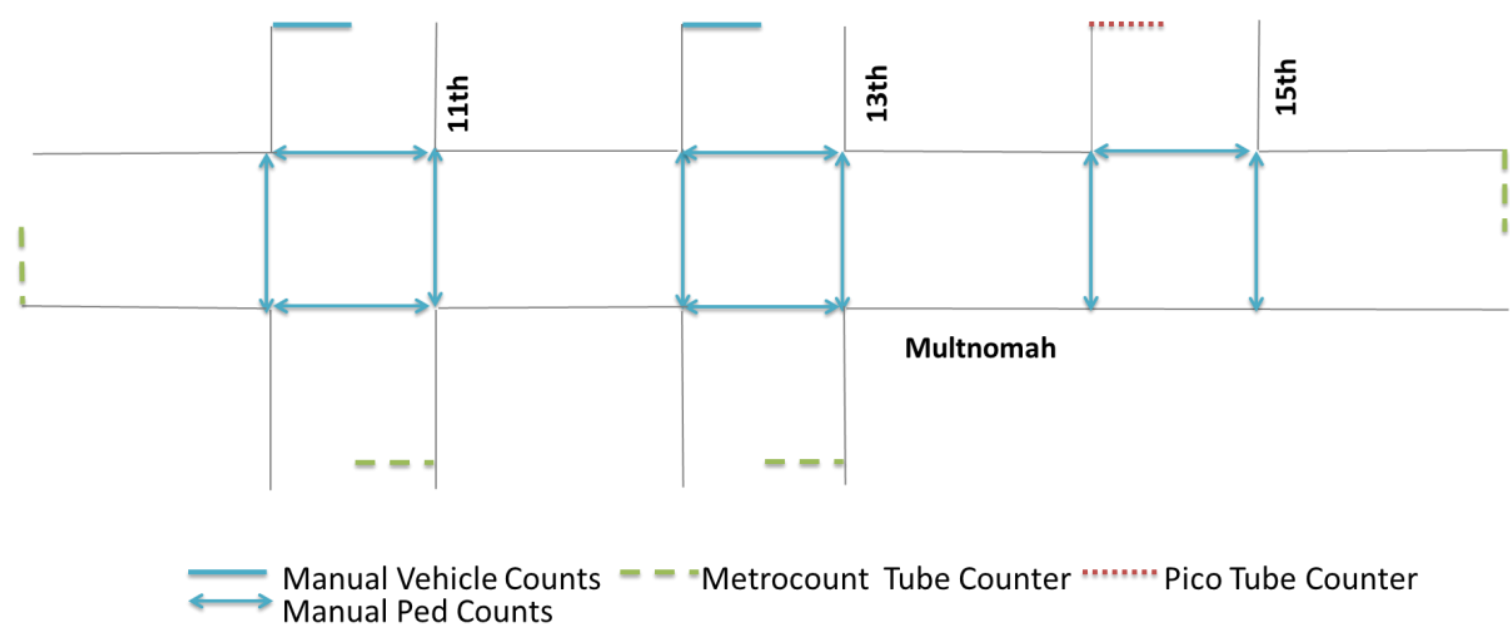

Figure 5-4 Bicycle and Vehicle Count Locations

The MetroCount ${ }^{\circledR}$ MC5600 system and associated software stores information on every axle hit and uses a classification scheme to differentiate vehicles into classes. Prior research by Boulder County, CO revealed that the classification scheme developed by Metro Count was not accurate for counting bicycles (Hyde-Wright et al., 2014). Their study found that Metro Count's software was either misclassifying groups of bicyclists as trucks or did not classify them at all (Hyde-Wright et al., 2014). Hence they developed a Boulder County modified classification theme (BOCO) that revised the rules for truck classes to exclude groups of bicyclists and created new classes for groups of bicyclists. The BOCO scheme was used in this study to gather bicycle and vehicle volumes. Figure 5-5 shows the light vehicle, heavy vehicle and bicycle volumes obtained from the tube counts. 
Multnomah EB Oct 3 - Oct 4, 2013

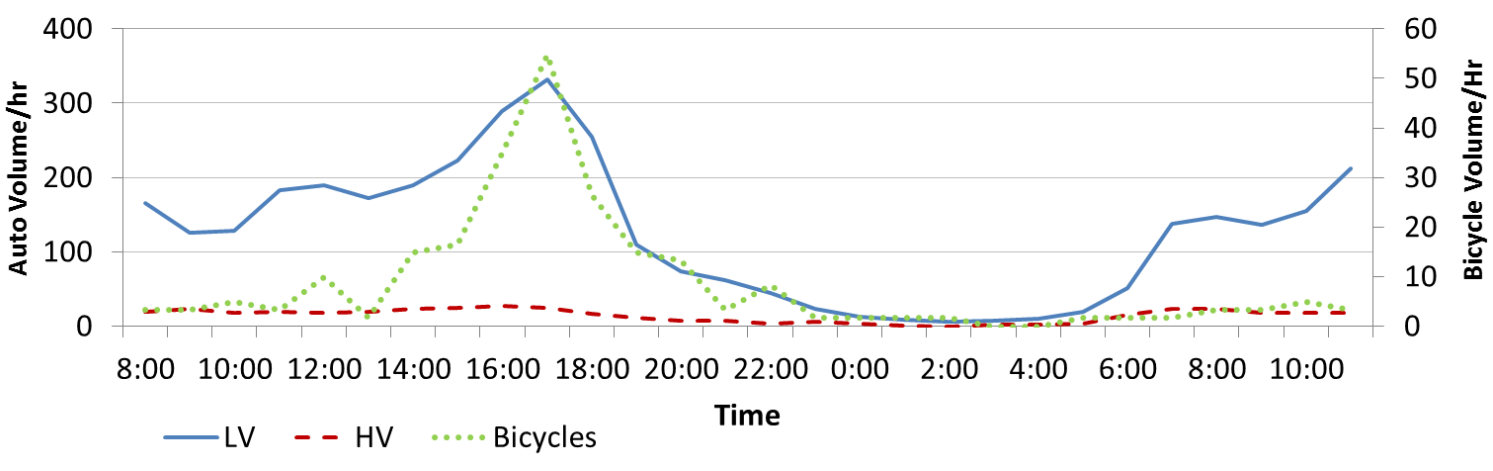

Multnomah WB Oct 3 - Oct 4, 2013

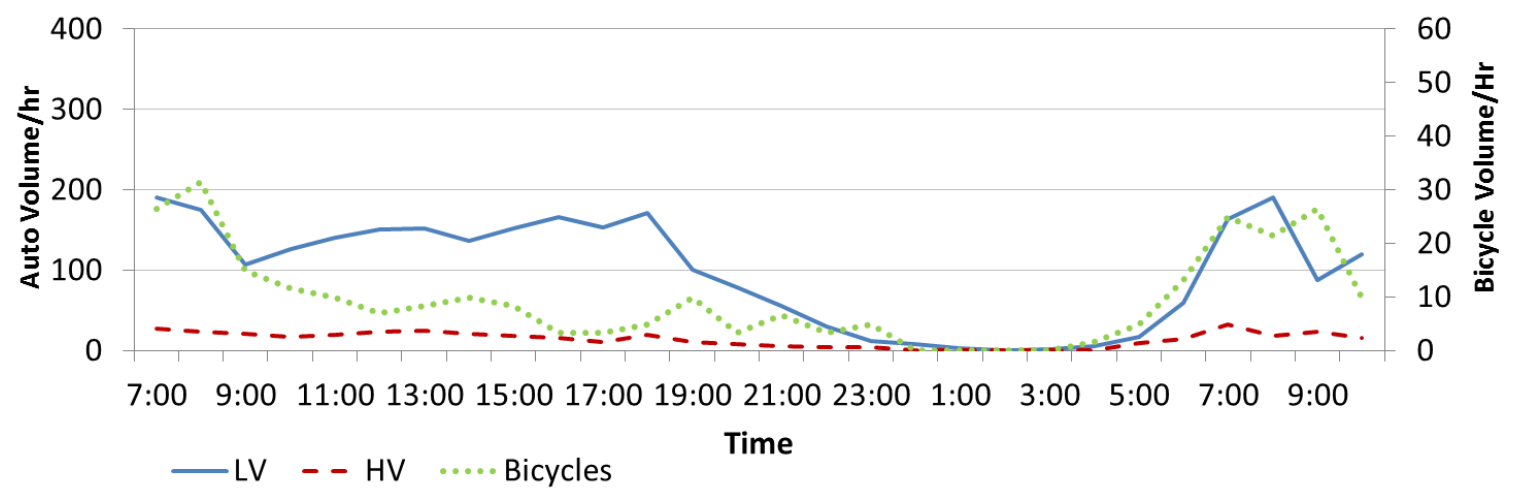

11th NB Oct 3 - Oct 4, 2013

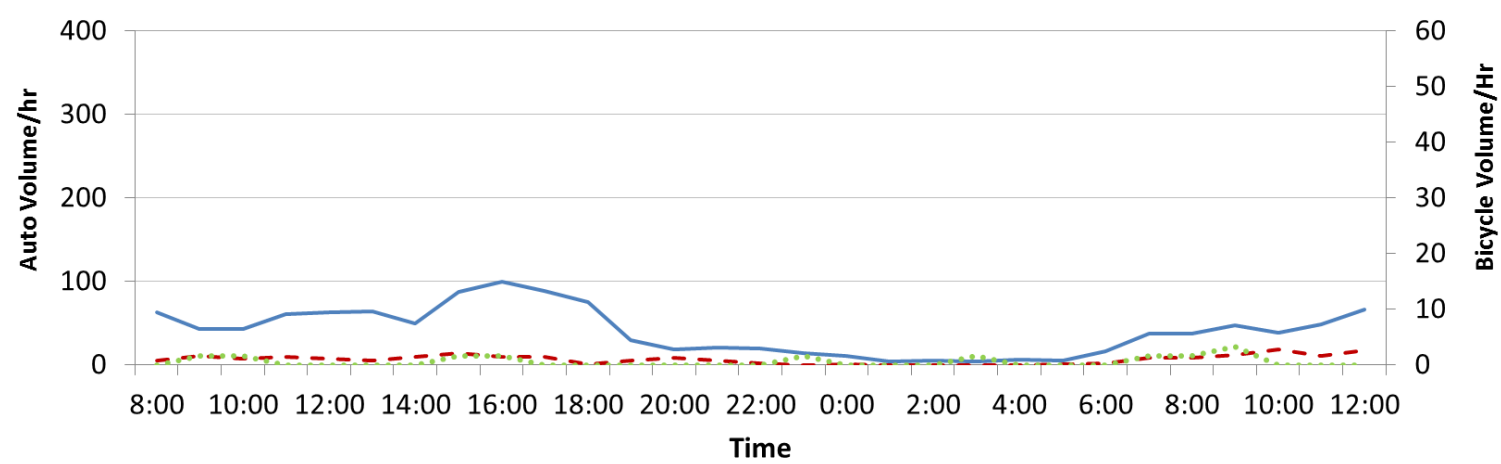

- LV - - HV ....B. Bicycles 
13th NB Oct 3-Oct 4, 2013

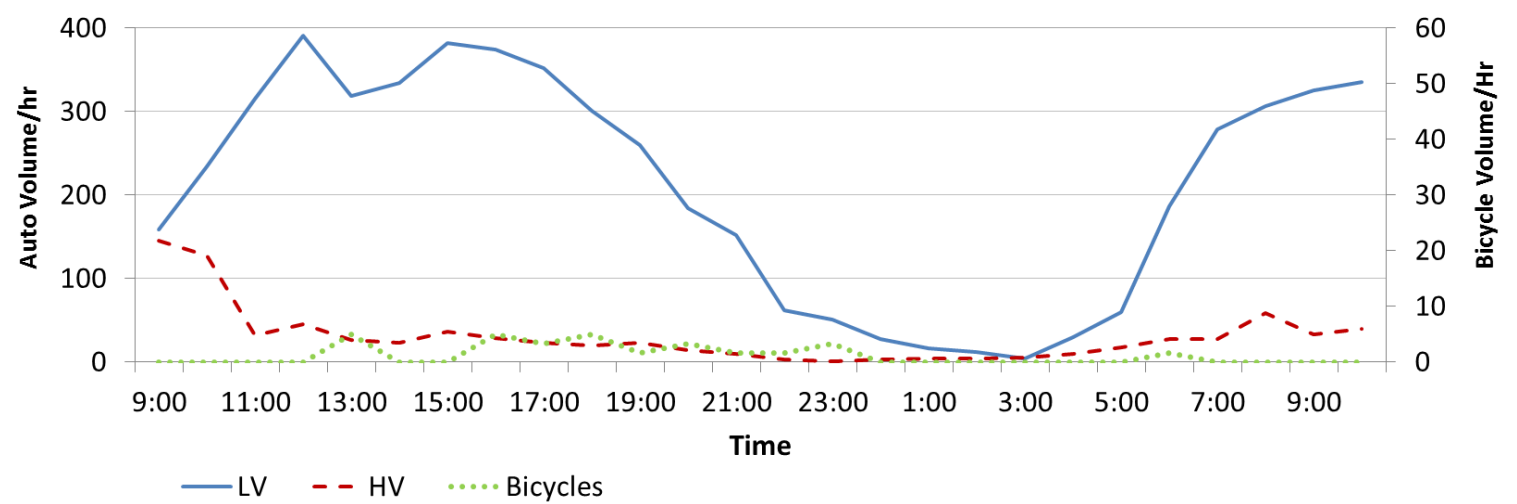

15th SB Oct 3 - Oct 4, 2013

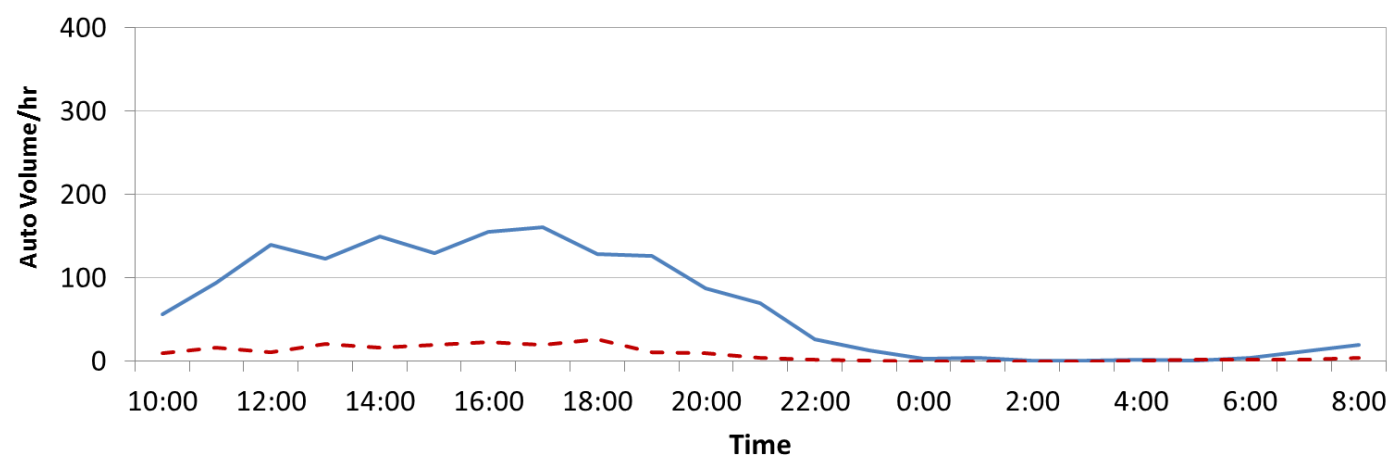

- LV $\quad--\mathrm{HV}$

Figure 5-5 Vehicle and Bicycle Volumes

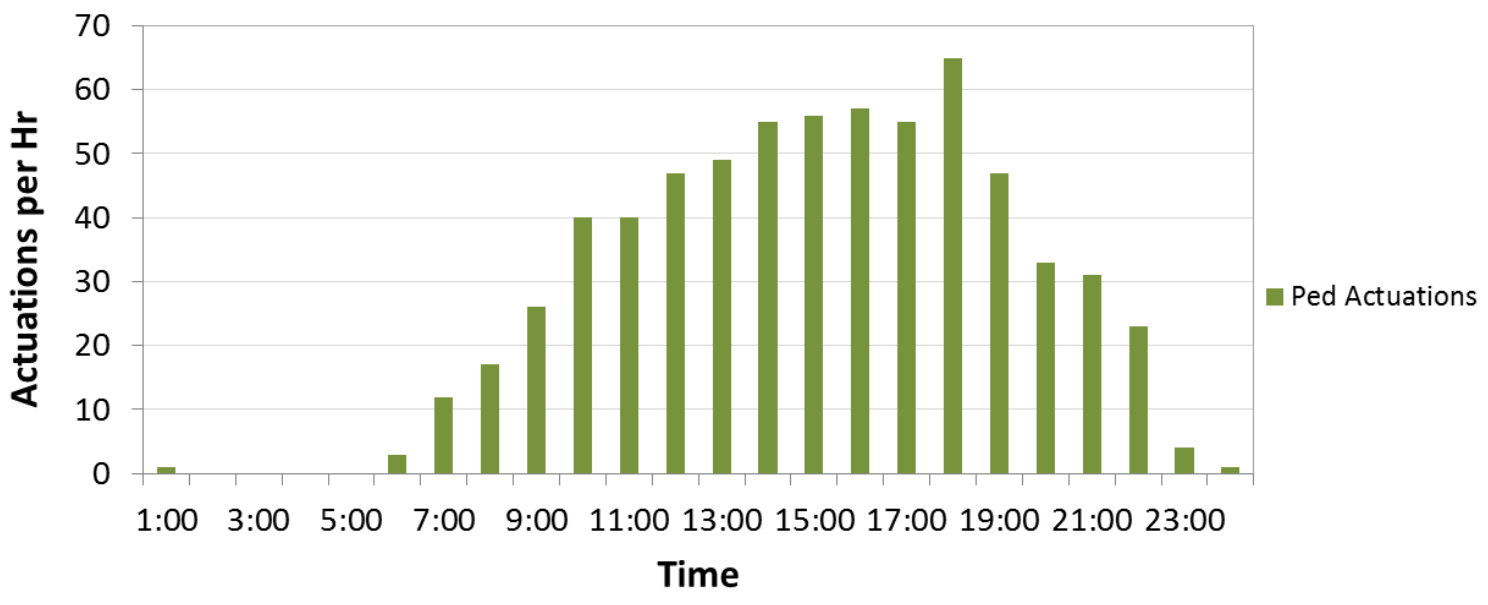

Figure 5-6 Pedestrian Actuations at Multnomah and $13^{\text {th }}$, October 3, 2013 
Figure 5-6 shows the pedestrian actuations on October 3, 2013 at Multnomah and $13^{\text {th }}$. The pedestrian actuations are higher in the afternoon, with highest actuations observed during the evening. Based on the volumes observed in Figure 5-5, three time periods were chosen for analysis: 7-8 AM, 12-1 PM and 4-6 PM. The chosen morning and evening time periods corresponded to the traditional traffic peak periods for vehicles. Although the noon hour did not have the highest pedestrian actuation activity, the vehicle volumes during this time period were low, therefore representing a scenario with low vehicle volumes and moderate pedestrian demand. Three simulation models were developed in VISSIM, one corresponding to each time period. For the two approaches (NE $11^{\text {th }}$ Ave SB, NE $13^{\text {th }}$ Ave SB) where flows were not captured using pneumatic tubes, manual observations of volumes for three hours were recorded. Pedestrian flows for all eleven crosswalks were also obtained manually for these three time periods. Pedestrian volume for each crosswalk was recorded manually using data collection sheets, by an observer standing at the intersection during each time period. These observations were collected during weekdays in October 2013. Table 5-1 shows the vehicle flows for all approaches for the three time periods.

Table 5-1 Vehicle Volumes per Hour per Approach

\begin{tabular}{l|rrr}
\hline Approach & $\begin{array}{r}\boldsymbol{A M} \\
(\mathbf{7 - 8} \boldsymbol{A M})\end{array}$ & $\begin{array}{r}\text { Mid-day } \\
(\mathbf{1 2 - 1} \boldsymbol{P M})\end{array}$ & $\begin{array}{r}\boldsymbol{P M} \\
(\mathbf{5 - 6} \boldsymbol{P M})\end{array}$ \\
\hline Multnomah EB & 161 & 208 & 357 \\
$11^{\text {th }} \mathrm{NB}$ & 45 & 70 & 97 \\
$11^{\text {th }} \mathrm{SB}$ & 0 & 52 & 60 \\
$13^{\text {th }} \mathrm{NB}$ & 305 & 436 & 375 \\
$13^{\text {th }} \mathrm{SB}$ & 0 & 5 & 12 \\
$15^{\text {th }} \mathrm{SB}$ & 14 & 151 & 181 \\
Multnomah WB & 218 & 175 & 164 \\
Total & $\mathbf{7 4 3}$ & $\mathbf{1 0 9 7}$ & $\mathbf{1 2 4 6}$ \\
\hline
\end{tabular}


The bicycle flows were obtained from the tube counts as shown in Figure 5-5. Here they are presented for the three analyzed time periods in Table 5-2. The pedestrian volumes for each intersection separated by direction are shown in Table 5-3.

Table 5-2 Bicycle Volumes on Multnomah Boulevard

\begin{tabular}{l|rrr}
\hline \multirow{2}{*}{ Approach } & $\begin{array}{r}\text { AM } \\
(7-8 \boldsymbol{A M})\end{array}$ & $\begin{array}{c}\text { Mid-day } \\
(\mathbf{1 2 - 1} \boldsymbol{P M})\end{array}$ & $\begin{array}{r}\boldsymbol{P M} \\
(\mathbf{5 - 6} \boldsymbol{P M})\end{array}$ \\
\hline Multnomah EB & 2 & 10 & 55 \\
Multnomah WB & 27 & 7 & 3 \\
Total & $\mathbf{2 9}$ & $\mathbf{1 7}$ & $\mathbf{5 8}$ \\
\hline
\end{tabular}

Table 5-3 Pedestrian Volumes

\begin{tabular}{l|rrrr|rrrr|rrrr}
\hline Intersection & \multicolumn{4}{|c|}{$\boldsymbol{A M}$} & \multicolumn{4}{|c|}{ Mid-day } & \multicolumn{4}{c}{$\boldsymbol{P M}$} \\
\hline & NB & SB & EB & WB & NB & SB & EB & WB & NB & SB & EB & WB \\
11th & 55 & 31 & 18 & 40 & 280 & 186 & 70 & 97 & 205 & 164 & 56 & 30 \\
13th & 26 & 32 & 21 & 44 & 138 & 133 & 95 & 72 & 110 & 144 & 89 & 68 \\
15th & 11 & 44 & 6 & 32 & 13 & 28 & 24 & 22 & 54 & 42 & 25 & 7 \\
Total & & & & $\mathbf{3 6 0}$ & & & & $\mathbf{1 1 5 8}$ & & & & $\mathbf{9 9 4}$ \\
\hline
\end{tabular}

Turning movement counts for each movement were obtained through manual observations for a 15 minute period for each study hour at each intersection. The 15 minute turning movement ratios were assumed to be constant for the entire hour and were then used to allocate the total volume per approach during each analyzed time period into specific movements. These ratios varied based on time of day.

Signal timing for each intersection was obtained from TransSuite ${ }^{\circledR}$, the central ATMS software for the City of Portland, Oregon. The signal timing plans yielded information on basic signal timing parameters such as minimum and maximum green time, pedestrian walk and clearance times, phase sequence, rotation and coordination schedule. The east and westbound movements along Multnomah St. were coordinated during all time periods. The cycle length at the three intersections varied between 70 and 
$80 \mathrm{sec}$. The $70 \mathrm{sec}$ cycle length was operational between $8 \mathrm{PM}-10 \mathrm{AM}$ and the $80 \mathrm{sec}$ cycle length was active during the rest of the day.

\subsubsection{Network Development}

The network for analysis was created in VISSIM. Road geometry was partly obtained from Google Maps ${ }^{\circledR}$ Satellite images. As mentioned earlier, this corridor was reconfigured in late 2012 by removing a traffic lane in each direction and through the addition of a cycle track. Although, the satellite images in Google Maps ${ }^{\circledR}$ were not updated to reflect the new configuration of the corridor with the cycle track, they were used to draw the general alignment of the corridor and spacing between intersections to scale. The width of each turn lane and through lane was coded as $10 \mathrm{ft}$, the width of the cycle track was coded as $7 \mathrm{ft}$ and the width of each pedestrian crosswalk was coded as 6 $\mathrm{ft}$. The vehicle speeds on Multnomah St. were obtained from the City of Portland's records and the speeds on the side streets were assumed. The speed of vehicles on Multnomah St. were coded to vary between $28-32 \mathrm{mph}$, the speeds on the side streets $\left(13^{\text {th }} \mathrm{NB}, 11^{\text {th }} \mathrm{NB}\right.$, and $\left.15^{\text {th }} \mathrm{SB}\right)$ were coded to vary between $23-27 \mathrm{mph}$ and exits from the mall $\left(11^{\text {th }} \mathrm{SB}\right.$ and $\left.13^{\text {th }} \mathrm{SB}\right)$ were coded to vary between $8-12 \mathrm{mph}$. Bicycle speeds were assumed to vary between 8 and $20 \mathrm{mph}$ and pedestrian speeds were assumed to vary between 3 - $5 \mathrm{mph}$. These speeds were obtained from other prior simulation models that were developed in the Portland metro region.

Based on the vehicle classification information from tube counts, the vehicle composition on Multnomah St. was coded as 90\% light vehicles and 10\% heavy vehicles. 
Similarly the composition on the side streets was coded as $91 \%$ light vehicles and $9 \%$ heavy vehicles. Auto, bicycle and pedestrian volumes as shown in Table 5-1, Table 5-2 and Table 5-3 were loaded onto the network. As the network was only approximately 0.3 miles in length, buses were ignored. Figure 5-7 shows the network in VISSIM.

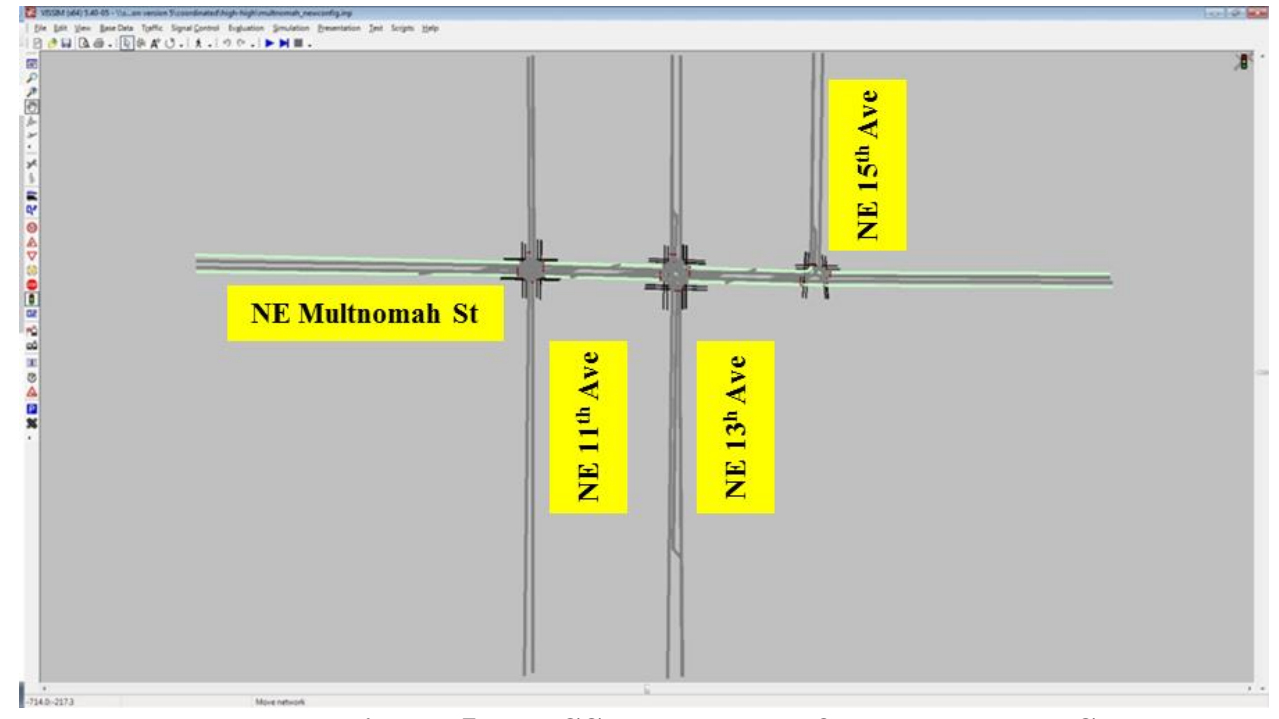

Figure 5-7 VISSIM Network for Multnomah Street

The Ring Barrier Controller (RBC) was used for coding signal timing at the intersections. This controller closely mimics the Voyage software used by the signal controllers at the City of Portland. The cycle lengths, splits, offsets and other signal timing parameters were obtained from current signal timing plans maintained by the City of Portland.

\subsubsection{Calibration}

Calibration is the process used to obtain a reliable model by specifying certain parameter values so that the model replicates local traffic conditions as accurately as possible (ODOT, 2011). The first step in the calibration process is to compare the input and output traffic volumes. In addition to volumes, ODOT's calibration guidelines suggest that other 
parameters such as travel times, spot speeds, congestion levels and duration, queue lengths and overall driver behavior should also be compared to field observations (ODOT, 2011).

\subsubsection{Volume Calibration}

In order to calibrate the models, simulation input and output volumes are compared to assess how closely they match. The GEH formula is the recommended metric to compare flows. The formula is given by:

$$
G E H=\sqrt{\frac{2(m-c)^{2}}{m+c}}
$$

where,

$\mathrm{m}=$ output traffic volume from simulation model (vph)

$\mathrm{c}=$ input traffic volume (vph)

ODOT VISSIM protocol report provides guidance on acceptable values for GEH statistic (ODOT, 2011). The criteria are listed in Table 5-4.

Table 5-4 GEH Criteria

\begin{tabular}{l|l}
\hline Value of Statistic & Criteria \\
\hline GEH $<5.0$ & Acceptable fit \\
$5.0<=\mathrm{GEH}<=10.0$ & Caution: possible model error or bad data \\
GEH $>10.0$ & Unacceptable \\
\hline
\end{tabular}

(Source: ODOT, Protocol for VISSIM Simulation, June 2011)

ODOT recommends that GEH statistics should be calculated for all intersection turns and mainline links and for traffic volumes at all entry and exit locations for each model and the criteria presented in Table 5-4 used to assess the validity of the model results. 
Table 5-5 GEH Statistic Calculations for Multnomah and $11^{\text {th }}$ using Mid-day Model

\begin{tabular}{|c|c|c|c|}
\hline Movement & Output & Input & $G E H$ \\
\hline EBLT & 61 & 58 & 0.39 \\
\hline EBTH & 131 & 129 & 0.18 \\
\hline EBRT & 22 & 21 & 0.22 \\
\hline SBLT & 31 & 29 & 0.37 \\
\hline SBTH & 8 & 7 & 0.37 \\
\hline SBRT & 16 & 16 & 0 \\
\hline WBLT & 48 & 45 & 0.44 \\
\hline WBTH & 262 & 259 & 0.19 \\
\hline WBRT & 15 & 16 & 0.25 \\
\hline NBLT & 34 & 30 & 0.35 \\
\hline NBTH & 12 & 11 & 0.29 \\
\hline NBRT & 28 & 27 & 0.19 \\
\hline EBTH Bike & 9 & 10 & 0.32 \\
\hline WBTH Bike & 7 & 7 & 0 \\
\hline East X-walk S-N Ped & 212 & 205 & 0.48 \\
\hline East X-walk N-S Ped & 102 & 103 & 0.10 \\
\hline South X-walk W-E Ped & 38 & 40 & 0.32 \\
\hline South X-walk E-W Ped & 32 & 34 & 0.35 \\
\hline West X-walk S-N Ped & 75 & 75 & 0 \\
\hline West X-walk N-S Ped & 82 & 83 & 0.11 \\
\hline North X-walk W-E Ped & 29 & 30 & 0.18 \\
\hline North X-walk E-W Ped & 65 & 63 & 0.25 \\
\hline Total & 1318 & 1300 & 0.50 \\
\hline
\end{tabular}

As stated earlier, three models based on AM, noon and PM volumes were developed.

Based on the above criteria, the input and output volumes for each individual model were compared using the GEH statistic. For every movement, calculated GEH statistic value was less than 5.0 thereby indicating that the models were acceptable. As an example, calculated GEH statistics values are shown in Table 5-5 for the intersection of Multnomah and $11^{\text {th }}$ during $12-1$ PM using the mid-day model.

\subsubsection{Travel Time Criteria}

Calibration criteria for travel times are listed in Table 5-6. The criteria suggest that modeled travel times from the simulation should be either within \pm 1 minute for short trips or within $\pm 15 \%$ of the observed travel times for longer trips. 
Table 5-6 Travel Time Criteria

\begin{tabular}{l|l}
\hline \multicolumn{1}{c|}{ Criteria } & \multicolumn{1}{c}{ Acceptance Targets } \\
\hline $\begin{array}{l}\text { Modeled travel time within }+1 \text { minute for routes observed } \\
\text { travel times less than } 7 \text { minutes }\end{array}$ & $\begin{array}{l}\text { All routes identified in the data collection } \\
\text { plan }\end{array}$ \\
$\begin{array}{l}\text { Modeled travel time within }+15 \% \text { for routes with observed } \\
\text { travel times greater than } 7 \text { minutes }\end{array}$ & $\begin{array}{l}\text { All routes identified in the data collection } \\
\text { plan }\end{array}$ \\
\hline
\end{tabular}

(Source: ODOT, Protocol for VISSIM Simulation, June 2011)

Travel times were measured using the floating car technique for the east and westbound through movements on NE Multnomah St. 9 travel time runs were conducted in the east bound direction and 10 travel time runs were conducted in the westbound direction.

Measured and simulated travel times are shown in Table 5-7. The mean travel time for the simulation is the average of 10 runs.

Table 5-7 Travel Time Calibration

\begin{tabular}{l|cr}
\hline & \multicolumn{3}{|c}{ Average Travel Time (s) } \\
\hline & Multnomah EB & Multnomah WB \\
Observed $(\mathrm{n}=9(\mathrm{~EB}), \mathrm{n}=10(\mathrm{WB}))$ & 59.46 & 75.5 \\
Simulated $(\mathrm{n}=10)$ & 63.26 & 82.35 \\
& Percent Difference & 0.09 \\
\hline
\end{tabular}

The percent difference between the observed and simulated travel times in the eastbound and westbound directions was $0.12 \%$ and $0.09 \%$ respectively. Since the travel time differences were within \pm 1 minute threshold, the model was deemed acceptable.

\subsubsection{Queuing}

ODOT guidelines recommend that queue lengths obtained from the model should also be compared to field observations to ensure that the intersection operation in the simulated model replicates the operation in the field. Excessive queuing or shorter queues may indicate errors in coding of signal timing or vehicle volumes (ODOT, 2011). The queue lengths are typically compared qualitatively. Queue lengths from the mid-day model were 
visually compared to field observations using qualitative measures and they matched well. The maximum and average queue lengths for through movements at each intersection during mid-day as obtained from the simulation model are shown in Table $5-8$.

Table 5-8 Queue Lengths for Through Movements during Mid-day

\begin{tabular}{r|rrr}
\hline Intersection & Approach & $\begin{array}{r}\text { Max Queue } \\
\text { Length (ft) }\end{array}$ & $\begin{array}{r}\text { Avg Queue } \\
\text { Length (ft) }\end{array}$ \\
\hline & EBTH & 112.66 & 6.36 \\
& WBTH & 180.65 & 19.33 \\
Multnomah and 11th & NBTH & 95.26 & 8.21 \\
& SBTH & 91.74 & 6.52 \\
\hline & EBTH & 117.28 & 8.89 \\
& WBTH & 182.71 & 20.99 \\
Multnomah and 13th & NBTH & 173.14 & 20.52 \\
& SBTH & 24.87 & 0.52 \\
\hline \multirow{3}{*}{ Multnomah and 15th } & EBTH & 100.85 & 5.36 \\
& WBTH & 140.06 & 8.17 \\
\hline
\end{tabular}

Once the models were calibrated, performance measures were extracted from each time of day model and are discussed below.

\subsection{Time of Day Model Results}

Three time of day models were run in VISSIM using the appropriate volumes and signal timing parameters. The signals were in coordination during all three time periods and the relevant cycle lengths, splits and offsets were coded in based on the existing signal timing plans. The main street pedestrian phase was placed in recall and rest in walk setting was also enabled to mimic the operation in the field. The rest in walk feature allows the pedestrian walk phase to expand during the coordinated movement green until a conflicting call on the side street is received. Side street pedestrian phases at Multnomah and $11^{\text {th }}$ and Multnomah and $15^{\text {th }}$ were also placed on pedestrian recall and side street 
phase at Multnomah and $15^{\text {th }}$ was placed on maximum recall to replicate the field settings.

Performance metrics such as overall average delay per person, average delay per person by mode, average stopped delay by mode, maximum and average queue lengths were extracted from the simulation. The results presented here represent an average of 10 simulation runs. Figure 5-8 shows the network volume by mode for each of the analyzed time periods.

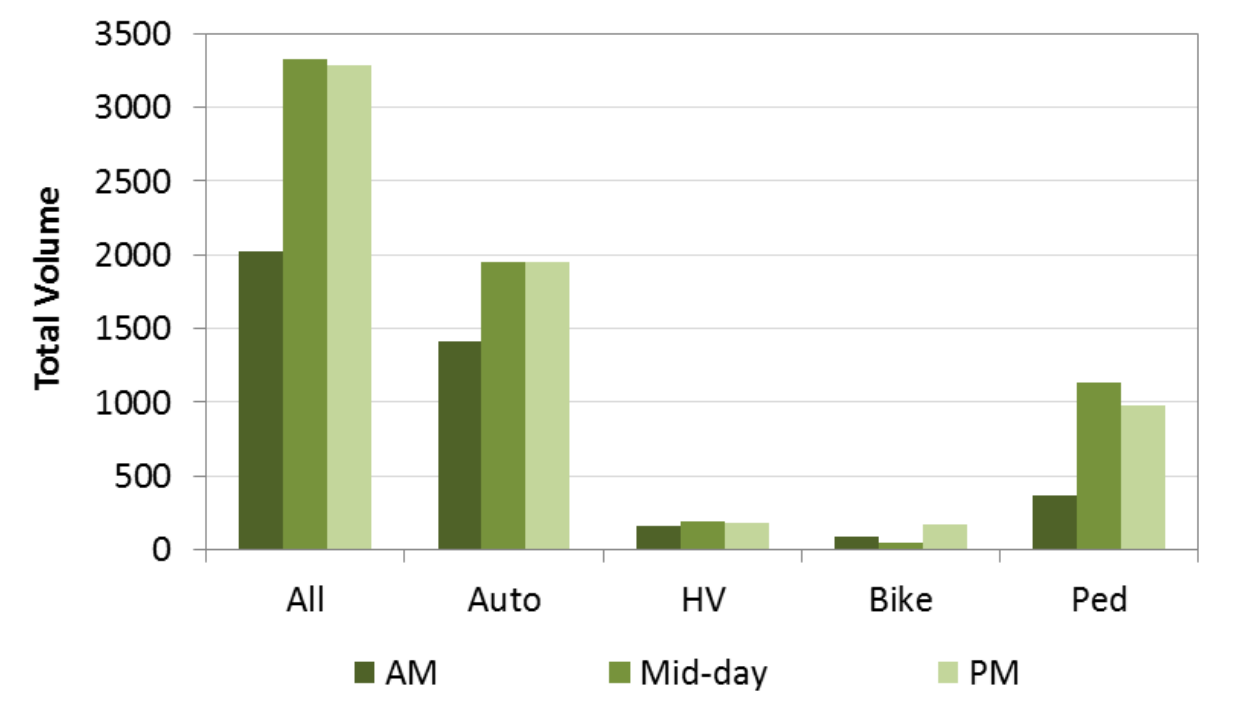

Figure 5-8 Network Volume by Mode

It is apparent from the above plot that the Multnomah corridor is busier during the midday and PM peak periods compared to the AM period. The highest percentages of pedestrians are observed during mid-day, when the ratio of pedestrians to vehicles (light and heavy) is 0.53 . The corresponding ratios of pedestrians to vehicles during the AM and PM peak periods are 0.23 and 0.45 . 
Average delays for the network were also extracted from the simulation models and are shown in Figure 5-9. The average delays for auto users were less than $20 \mathrm{sec}$ during all the analyzed time periods, with the delays during AM and PM peaks slightly lower than mid-day. For pedestrians however, the delay is lowest during the AM peak and increases during the mid-day and PM peak and approaches the 30s threshold. Bicycle delays are lower than any other mode because only the through bicycles on Multnomah Street are simulated in this research and as such they benefit from the green band and progression during coordination.

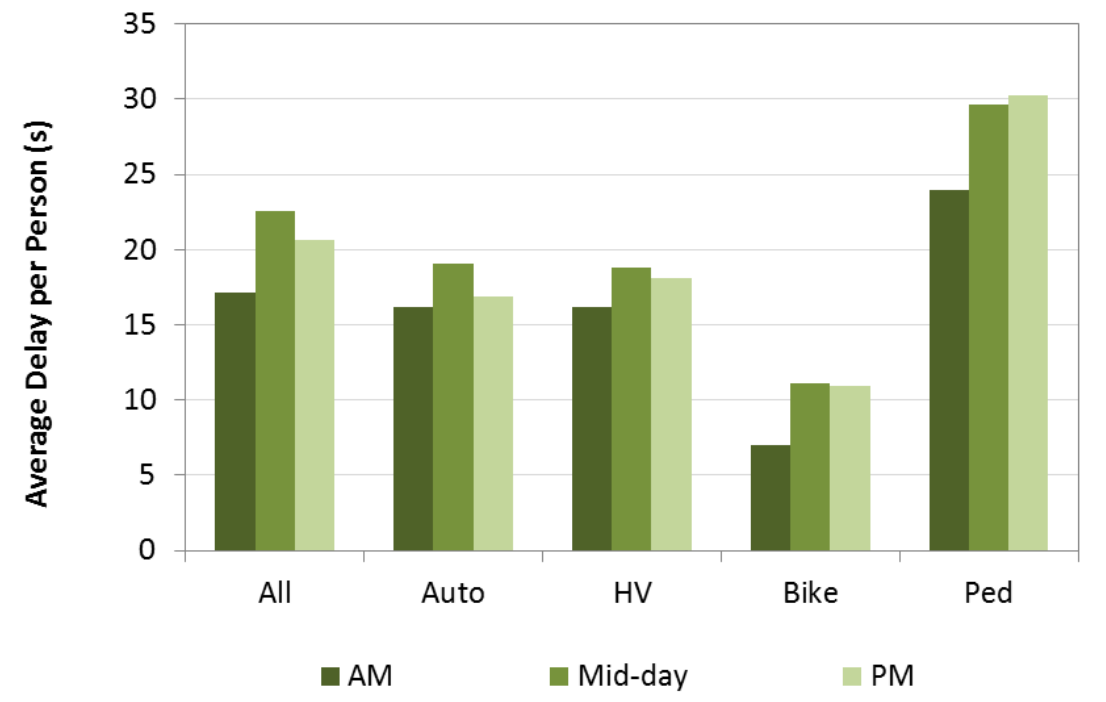

Figure 5-9 Average Delays per Person by Mode

Other performance metrics by time of day are shown in Table 5-9. In the existing configuration, the average stopped delay is highest for pedestrians and lowest for bikes during all analyzed time periods. The average number of stops also follows a similar trend. 
Table 5-9 Performance Measures by Time of Day

\begin{tabular}{l|rrr}
\hline \multicolumn{1}{r|}{ Performance Measure } & AM & Mid-day & PM \\
\hline Total Number of People & 2024.9 & 3325.80 & 3285.10 \\
Average Stopped Delay (All modes) (s) & 12.83 & 19.11 & 17.36 \\
Average Stopped Delay per LV (s) & 11.06 & 14.54 & 12.72 \\
Average Stopped Delay per HV (s) & 9.84 & 12.94 & 12.38 \\
Average Stopped Delay per Bike (s) & 5.68 & 9.47 & 9.27 \\
Average Stopped Delay per Ped (s) & 22.77 & 28.40 & 28.98 \\
Average No. of Stops (All modes) & 0.66 & 0.68 & 0.64 \\
Average No. of Stops per Vehicle (LV) & 0.65 & 0.61 & 0.57 \\
Average No. of Stops per Vehicle (HV) & 0.57 & 0.58 & 0.54 \\
Average No. of Stops per Vehicle (Bike) & 0.42 & 0.55 & 0.54 \\
Average No. of Stops per Vehicle (Ped) & 0.8 & 0.83 & 0.84 \\
Max Queue (ft) & 302.00 & 253.94 & 201.15 \\
\hline
\end{tabular}

The distribution of average green times for each intersection and phase for the three time periods are shown in Figure 5-10, Figure 5-11 and Figure 5-12. The distribution shows that in general average green times for the coordinated phases increase during mid-day and PM, due to higher traffic demands as well as longer cycle lengths. The exception to this rule is Multnomah and $13^{\text {th }}$, where due to the heavy traffic demand on the side street phase 8 (northbound $13^{\text {th }}$ Ave), the coordinated movement average green times decrease during the mid-day and PM peak periods compared to the AM peak period. 


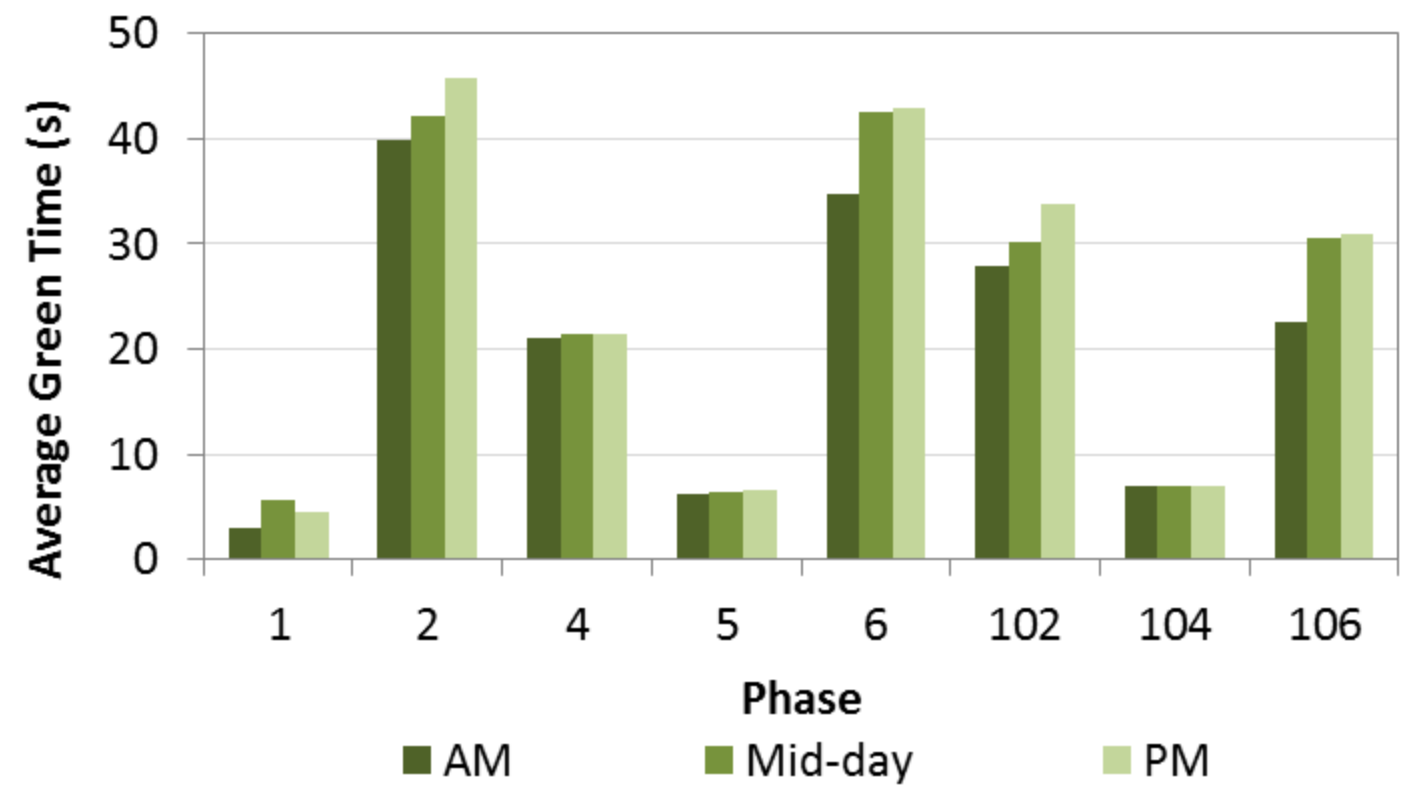

Figure 5-10 Average Green Times at Multnomah and $11^{\text {th }}$

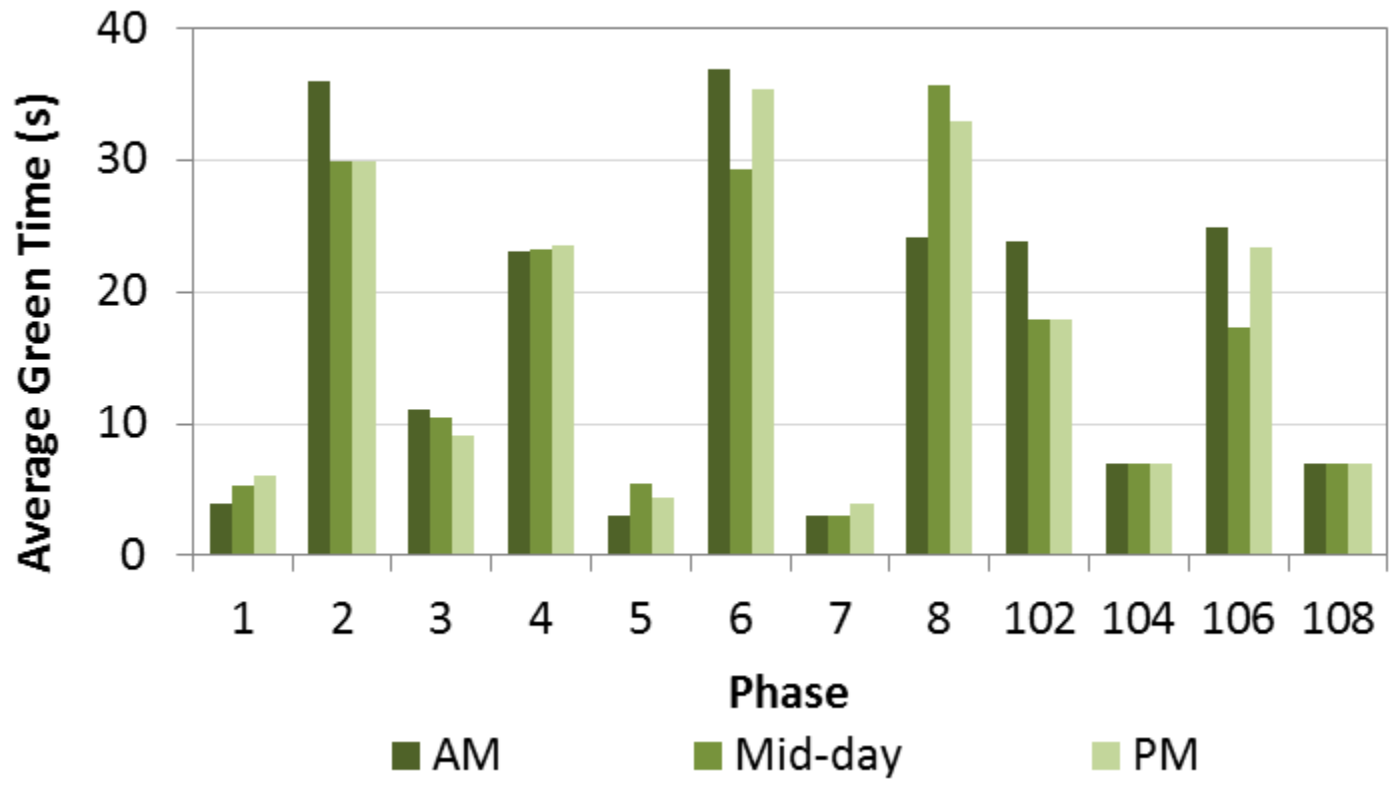

Figure 5-11 Average Green Times at Multnomah and $13^{\text {th }}$ 


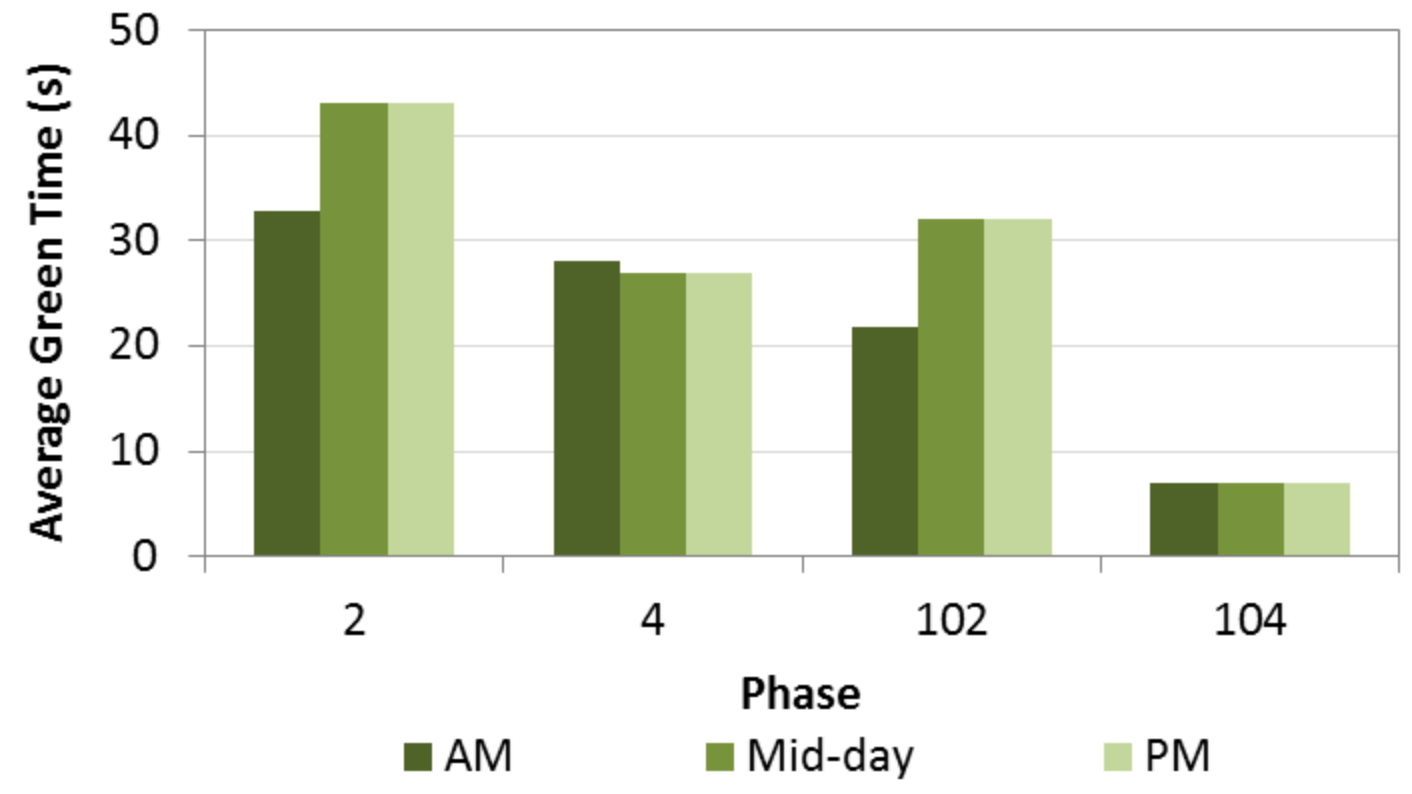

Figure 5-12 Average Green Times at Multnomah and $15^{\text {th }}$

As seen from Figure 5-8, pedestrians form a significant proportion of the traffic along this corridor during mid-day and PM peak periods. During AM and PM peak periods, traffic demand on Multnomah St. is higher than the side street. However during mid-day traffic demand on NE $13^{\text {th }}$ Ave is higher than traffic demand on Multnomah St. Therefore any strategies that are employed to benefit the side street pedestrians will also help the side street traffic.

In order to study the impacts of pedestrian friendly strategies such as changing the mode of operation from coordinated to free, certain hypothetical scenarios with varying pedestrian and auto volumes were constructed and these are presented in the next section. 


\subsection{Hypothetical Network Analysis}

A hypothetical network based on the validated Multnomah Street network was used to study the impacts on delay resulting from change in signal controller mode of operation from coordinated to free. The use of the hypothetical network allowed the flexibility to test the effects of semi-actuated and fully actuated free operation on pedestrian delay. Scenarios pertaining to coordinated and free operation were tested with varying vehicular and pedestrian demands to determine the traffic regimes where each mode of operation would be best suited. The metric for determining feasibility of mode of operation (coordinated or free) was minimization of overall network delay.

\subsubsection{Hypothetical Network}

While the majority of the Multnomah St. network features were carried over to the hypothetical network, a few changes were made. The assumptions made for the hypothetical model are described in this section. All Multnomah pedestrian movements are placed on recall and rest-in-walk enabled for coordinated operation. This was unchanged from the Multnomah St. network. All side street vehicle movements are actuated. Previously in the Multnomah St. network, some of these movements did not have detection and were placed on recall. All side street pedestrian movements are actuated. Previously, some of these movements were placed on recall in the original Multnomah St. network. The vehicle, bicycle and pedestrian flows were varied in three ranges of high, medium and low demand. The medium and low volumes were assumed 
to be $60 \%$ and $30 \%$ of the high volume respectively. In order to determine the high auto volumes, the capacity for each lane group was estimated, which is given by:

$$
c_{i}=s_{i} \frac{g_{i}}{C}
$$

where,

$\mathrm{c}_{\mathrm{i}}=$ capacity of lane group $\mathrm{i}(\mathrm{veh} / \mathrm{hr})$

$\mathrm{s}_{\mathrm{i}}=$ saturation flow rate for lane group $\mathrm{i}(\mathrm{veh} / \mathrm{hr})$

$\mathrm{g}_{\mathrm{i}} / \mathrm{C}=$ effective green ratio for lane group $\mathrm{i}$

The volume to capacity ratio for the lane group is calculated as

$$
X_{i}=\frac{v}{c_{i}}=\frac{v}{s_{i} g_{i}}=\frac{v_{i} C}{s_{i} g_{i}}
$$

where,

$\mathrm{X}_{\mathrm{i}}=\left(\mathrm{v} / \mathrm{c}_{\mathrm{i}}\right)=$ ratio for lane group $\mathrm{i}$,

$\mathrm{v}_{\mathrm{i}}=$ actual or projected demand flow rate for lane group i (veh/hr)

$\mathrm{s}_{\mathrm{i}}=$ saturation flow rate for lane group i (veh/hr)

$\mathrm{g}_{\mathrm{i}}=$ effective green time for lane group i (sec)

$\mathrm{C}=$ cycle length $(\mathrm{sec})$

The volumes for each lane group for the high volume scenario were assumed such that a $\mathrm{v} / \mathrm{c}$ ratio of 0.7 or greater was achieved for the coordinated movements (through movements). The exception to that rule was Multnomah and $15^{\text {th }}$, where the $\mathrm{v} / \mathrm{c}$ ratio for high scenario was $0.5-0.6$, due to only two phases being operational at the intersection, which in turn resulted in more green time for the coordinated movement. The $\mathrm{v} / \mathrm{c}$ ratios 
for the coordinated movements for high, medium and low volume scenarios at the three intersections are shown in Table 5-10.

Table 5-10 V/C Ratios for Different Scenarios

\begin{tabular}{l|rr|rr|rr}
\hline \multicolumn{1}{c|}{ Intersection } & \multicolumn{2}{|c|}{ High } & \multicolumn{2}{c|}{ Medium } & \multicolumn{2}{c}{ Low } \\
\hline & EB & WB & EB & WB & EB & WB \\
Multnomah and 11th & 0.73 & 0.71 & 0.43 & 0.44 & 0.24 & 0.22 \\
Multnomah and 13th & 0.96 & 0.77 & 0.57 & 0.47 & 0.29 & 0.23 \\
Multnomah and 15th & 0.6 & 0.5 & 0.36 & 0.3 & 0.18 & 0.15 \\
\hline
\end{tabular}

Pedestrian volumes were also divided into three ranges of high, medium and low. Based on the input volume provided by the user, VISSIM loads the pedestrians onto crosswalks in a random manner using the Poisson distribution. The volumes were assumed based on the frequency of pedestrian phases in an hour. In the high scenario, the demand was assumed such that a pedestrian phase would come up every cycle. The pedestrian volume was obtained by observing multiple simulation runs with varying pedestrian volumes and determining the number of cycles during which the pedestrian phase was served. For example, the maximum number of cycles in one hour with a $80 \mathrm{sec}$ cycle length is 45 (3600/80). Assuming a pedestrian volume of 150 per crosswalk, the number of pedestrian phases served in one hour based on observation of multiple VISSIM runs is 45. This implies that the pedestrian phase is served every cycle and the frequency is $100 \%$. In the medium and low scenarios, the pedestrian phase was designed to be served during approximately $60 \%$ and $30 \%$ of the number of cycles in one hour. Table 5-11 shows the varying pedestrian flows for the high, medium and low scenarios. 
Table 5-11 Pedestrian Flows for Different Scenarios

\begin{tabular}{l|rrr}
\hline & High & Medium & Low \\
\hline Ped Volume/X-Walk & 150 & 50 & 10 \\
\# of Cycles (80 s CL) & 45 & 45 & 45 \\
\# of Observed Ped Phases & 45 & 29 & 10 \\
Ped Phase Frequency (\# & $100 \%$ & $64 \%$ & $22 \%$ \\
Ped Phases/\# Cycles) & & & \\
\hline
\end{tabular}

The vehicle, bicycle and pedestrian volumes for the high scenario are shown in Figure 5-13. The bicycle volumes are in red (bold). As stated earlier, the medium and low volumes for the auto and bicycle were assumed to $60 \%$ and $30 \%$ of the high volume respectively.

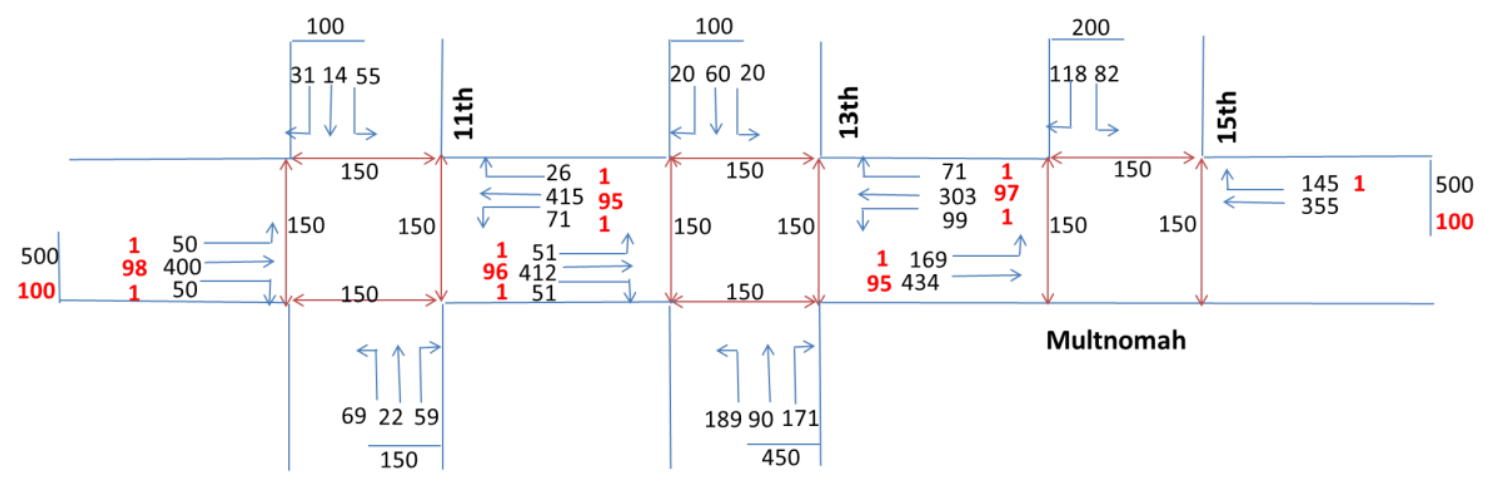

Figure 5-13 Auto, Bicycle and Pedestrian Volumes in the High Scenario

The speeds and vehicle compositions were left unchanged. The mid-day Multnomah signal timings including cycle length, splits and offsets were assumed for all scenarios.

\subsubsection{Simulation Parameters}

Based on the varying combinations of auto and pedestrian volumes, a total of 18 simulation models were constructed; 9 for each mode of operation. Figure 5-14 shows a graphic of the various simulation models that were developed. 


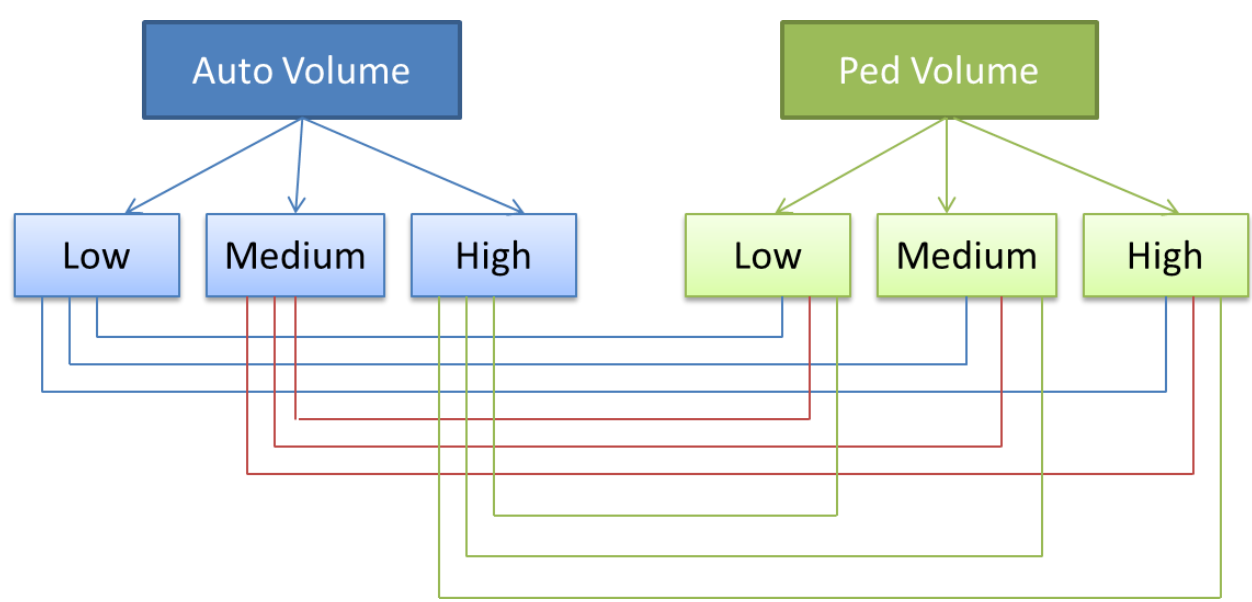

Figure 5-14 Simulation Models

For each combination of auto and pedestrian volume, 10 runs were carried out. The number of runs was selected based on the recommendation in ODOT's VISSIM protocol guide (ODOT, 2011). Using a random number generator, a starting random seed was generated. Since there were 18 different flow combinations, a total of 180 simulation runs were created. Each run was approximately 75 minutes long and the data from the first 15 minutes was discarded for analysis purposes as the network was still being populated during this time. The simulation resolution was set to 10 time steps/s similar to the calibrated Multnomah St. network.

\subsubsection{Results}

The resulting simulation outputs were analyzed and performance metrics were extracted similar to original Multnomah Street network. Average delay per user was the metric used to assess the performance of the mode of operation. Delays for coordinated operation were compared to the delays resulting from free operation using Welch's two sample T-Test with unequal variances. The t-statistic is given by the following formula 


$$
\mathrm{t}=\frac{\overline{\mathrm{X}}_{1}-\overline{\mathrm{X}}_{2}}{\sqrt{\frac{\mathrm{s}_{1}^{2}}{\mathrm{~N}_{1}}+\frac{\mathrm{s}_{2}^{2}}{\mathrm{~N}_{2}}}}
$$

where,

$\overline{\mathrm{X}}_{\mathrm{i}}=$ sample mean

$\mathrm{s}_{\mathrm{i}}^{2}=$ sample variance

$\mathrm{N}_{\mathrm{i}}=$ sample size

The null hypothesis in this analysis is that the two means are equal. The p-value is estimated for each scenario and if p-value is less than level of confidence $(\alpha=0.05)$, the null hypothesis is rejected. For all the nine scenarios, overall average delay per user was significantly different for the two operations. The average delays for each scenario along with the corresponding $p$-values are provided in the appendix.

Table 5-12 shows the performance metrics for the low auto volumes with varying pedestrian demand. The percent difference in delay is calculated as the ratio of difference in delay between the free mode and coordinated mode to the coordinated delay as shown below:

$$
\% \text { Diff in Delay }=\frac{(\text { Free Delay-Coordinated Delay }) * 100}{\text { Coordinated Delay }}
$$


Table 5-12 Performance Metrics for Low Auto and Varying Pedestrian Volumes

\begin{tabular}{l|rrr}
\hline & \multicolumn{3}{|c}{ \% Difference } \\
& \multicolumn{4}{|c}{$(($ Free Metric-Coord Metric)*100)/Coord Metric) } \\
& Low Auto-Low Ped & Low Auto-Med Ped & Low Auto-High Ped \\
\hline Avg. Person Delay(All) (s) & $\mathbf{- 3 2 . 3 7}$ & $\mathbf{- 1 4 . 3 6}$ & $\mathbf{- 1 0 . 8 7}$ \\
Avg. LV Delay (s) & $\mathbf{- 3 6 . 6 6}$ & $\mathbf{- 1 6 . 4 4}$ & $\mathbf{- 2 . 6 4}$ \\
Avg. HV Delay (s) & $\mathbf{- 3 3 . 6 3}$ & $\mathbf{- 1 6 . 7 0}$ & $\mathbf{- 3 . 4 2}$ \\
Avg. Bike Delay (s) & $-\mathbf{1 0 . 9 3}$ & $\mathbf{1 9 . 2 3}$ & $\mathbf{2 7 . 3 0}$ \\
Avg. Ped Delay (s) & $-\mathbf{1 7 . 1 2}$ & $\mathbf{- 1 6 . 8 4}$ & $\mathbf{- 1 5 . 1 3}$ \\
Avg. Stopped Delay(All) (s) & $-\mathbf{4 4 . 1 8}$ & $\mathbf{- 2 0 . 5 4}$ & $\mathbf{- 1 5 . 3 8}$ \\
Avg. LV Stopped Delay (s) & $-\mathbf{5 1 . 4 6}$ & $\mathbf{- 2 7 . 5 9}$ & $\mathbf{- 1 3 . 0 8}$ \\
Avg. HV Stopped Delay (s) & $-\mathbf{5 3 . 2 1}$ & $\mathbf{- 3 2 . 7 1}$ & $\mathbf{- 1 6 . 0 4}$ \\
Avg. Bike Stopped Delay (s) & $-\mathbf{1 9 . 1 4}$ & $\mathbf{1 5 . 5 6}$ & $\mathbf{2 3 . 8 4}$ \\
Avg. Ped Stopped Delay (s) & $\mathbf{- 1 8 . 9 7}$ & $\mathbf{- 1 8 . 2 3}$ & $\mathbf{- 1 6 . 4 2}$ \\
Avg. \# of Stops(All) & $\mathbf{9 . 6 9}$ & $\mathbf{1 4 . 8 7}$ & $\mathbf{1 6 . 9 8}$ \\
Avg. \# of Auto Stops & $\mathbf{7 . 6 2}$ & $\mathbf{1 2 . 2 9}$ & $\mathbf{2 2 . 9 1}$ \\
Avg. \# of HV Stops & $-\mathbf{5 . 0 8}$ & $\mathbf{8 . 0 7}$ & $\mathbf{1 5 . 9 8}$ \\
Avg. \# of Bike Stops & $\mathbf{1 2 . 2 7}$ & $\mathbf{3 5 . 8 7}$ & $\mathbf{4 6 . 9 4}$ \\
Avg. \# of Ped Stops & $\mathbf{2 5 . 9 1}$ & $\mathbf{1 3 . 5 1}$ & $\mathbf{1 2 . 9 8}$ \\
Max Queue (ft) & $\mathbf{- 1 5 . 2 8}$ & $\mathbf{- 8 . 3 5}$ & $\mathbf{1 3 . 8 4}$ \\
\hline
\end{tabular}

The negative values in Table 5-12 indicate that free operation delay is lower than coordinated delay. For low auto volumes and varying pedestrian volumes (low, medium or high), the average delay per user when all modes are considered is lower when the signal is operating in free mode compared to the coordinated operation as seen in the above table. The reduction in delay ranged from $32.37 \%$ for the low auto- low ped scenario to $10.87 \%$ for the low auto-high ped scenario. Average delay per user for autos, heavy vehicles and pedestrians was lower for all three scenarios, however average delay for bicyclists increased during free operation for the low auto-medium ped and low autohigh ped scenarios. As the volume of pedestrians increases, the signal is likely to be more responsive to side street pedestrians during free operation. Since the bicycle 
movements on this network are coded as only through movements on the main line, their delay increases due to lack of a green band for progression through the corridor during free operation. The impact of the lack of progression is also seen in the difference between the average number of stops for bicyclists during coordinated and free operation. The percent increase in the average number of stops for a bicyclist ranges from 12.27 for low auto-low ped scenario to 46.94 for the low auto-high ped scenario.

Table 5-13 shows the performance metrics for medium auto volumes $(0.3<\mathrm{v} / \mathrm{c}<$ 0.7 ) and varying pedestrian volumes. The trend seen is very similar to the low auto volume scenario, where free operation produced lower overall average delay per user compared to the coordinated operation. Average delay per bicyclist increased for free operation due to the reasons outlined earlier. Average number of stops also increased per user for all modes during free operation. 
Table 5-13 Performance Metrics for Medium Auto and Varying Pedestrian Volumes

\begin{tabular}{l|rrr}
\hline & \multicolumn{3}{|c}{ \% Difference } \\
& Med Auto-Low Ped & Med Auto-Med Ped & Med Auto-High Ped \\
\hline Avg. Person Delay(All) (s) & $\mathbf{- 2 0 . 1 6}$ & $\mathbf{- 8 . 7 9}$ & $\mathbf{- 7 . 0 5}$ \\
Avg. LV Delay (s) & -23.02 & $-\mathbf{8 . 3 2}$ & $\mathbf{- 2 . 0 6}$ \\
Avg. HV Delay (s) & $-\mathbf{2 0 . 9 2}$ & $\mathbf{- 1 0 . 5 5}$ & $\mathbf{- 4 . 4 4}$ \\
Avg. Bike Delay (s) & $\mathbf{1 5 . 1 5}$ & $\mathbf{1 4 . 7 9}$ & $\mathbf{1 1 . 2 9}$ \\
Avg. Ped Delay (s) & $-\mathbf{1 6 . 9 3}$ & $\mathbf{- 1 5 . 2 9}$ & $\mathbf{- 1 2 . 6 4}$ \\
Avg. Stopped Delay(All) (s) & $\mathbf{- 3 3 . 2 1}$ & $\mathbf{- 1 6 . 9 7}$ & $\mathbf{- 1 2 . 5 7}$ \\
Avg. LV Stopped Delay (s) & $\mathbf{- 3 7 . 4 7}$ & $\mathbf{- 1 9 . 4 1}$ & $\mathbf{- 1 2 . 1 4}$ \\
Avg. HV Stopped Delay (s) & $-\mathbf{4 1 . 2 2}$ & $\mathbf{- 2 5 . 7 5}$ & $\mathbf{- 1 6 . 5 0}$ \\
Avg. Bike Stopped Delay (s) & $\mathbf{1 0 . 2 8}$ & $\mathbf{1 0 . 8 9}$ & $\mathbf{7 . 0 5}$ \\
Avg. Ped Stopped Delay (s) & $-\mathbf{1 8 . 8 6}$ & $\mathbf{- 1 6 . 6 3}$ & $\mathbf{- 1 3 . 6 9}$ \\
Avg. \# of Stops(All) & $\mathbf{1 6 . 4 5}$ & $\mathbf{1 7 . 2 0}$ & $\mathbf{1 6 . 5 4}$ \\
Avg. \# of Auto Stops & $\mathbf{1 4 . 0 4}$ & $\mathbf{1 6 . 3 8}$ & $\mathbf{1 9 . 5 5}$ \\
Avg. \# of HV Stops & $\mathbf{6 . 2 5}$ & $\mathbf{8 . 8 1}$ & $\mathbf{1 4 . 3 4}$ \\
Avg. \# of Bike Stops & $\mathbf{4 1 . 9 4}$ & $\mathbf{3 6 . 7 8}$ & $\mathbf{3 8 . 7 1}$ \\
Avg. \# of Ped Stops & $\mathbf{2 2 . 4 4}$ & $\mathbf{1 4 . 2 1}$ & $\mathbf{1 0 . 7 1}$ \\
Max Queue (ft) & $\mathbf{9 . 3 7}$ & $\mathbf{8 . 6 7}$ & $\mathbf{9 . 8 6}$ \\
\hline
\end{tabular}

The performance metrics for the high auto volumes (v/c $>0.7)$ are shown in Table 5-14.

While the average delay per user for the high auto-low ped is still lower during free operation, the trend reverses for the high auto-medium ped and high auto-high ped scenarios. The average delays per pedestrian are always lower for free operation in the scenarios tested here. However, the delays for the other modes increase during free operation with higher auto volumes. For the high auto-high ped scenario, the average auto delay per user increases $49 \%$ and average bicycle delay increases $15.78 \%$ during free operation. 
Table 5-14 Performance Metrics for High Auto and Varying Pedestrian Volumes

\begin{tabular}{l|rrr}
\hline & \multicolumn{3}{|c}{ \% Difference } \\
& $(($ Free Metric-Coord Metric)*100)/Coord Metric) \\
\hline & High Auto-Low Ped & High Auto-Med Ped & High Auto-High Ped \\
Avg. Person Delay(All) (s) & $\mathbf{- 9 . 9 6}$ & $\mathbf{8 . 5 3}$ & $\mathbf{2 7 . 7 6}$ \\
Avg. LV Delay (s) & $\mathbf{- 1 0 . 5 8}$ & $\mathbf{1 2 . 2 3}$ & $\mathbf{4 9 . 1 4}$ \\
Avg. HV Delay (s) & $-\mathbf{1 1 . 5 2}$ & $\mathbf{1 3 . 3 8}$ & $\mathbf{4 2 . 6 9}$ \\
Avg. Bike Delay (s) & $\mathbf{4 . 0 6}$ & $\mathbf{1 1 . 9 8}$ & $\mathbf{1 5 . 7 8}$ \\
Avg. Ped Delay (s) & $\mathbf{- 1 5 . 4 2}$ & $\mathbf{- 1 1 . 4 3}$ & $\mathbf{- 7 . 0 0}$ \\
Avg. Stopped Delay(All) (s) & $\mathbf{2 2 . 8 7}$ & $\mathbf{- 1 . 3 5}$ & $\mathbf{1 7 . 7 2}$ \\
Avg. LV Stopped Delay (s) & $\mathbf{2 4 . 1 5}$ & $\mathbf{0 . 8 5}$ & $\mathbf{3 8 . 8 0}$ \\
Avg. HV Stopped Delay (s) & $\mathbf{2 9 . 0 7}$ & $\mathbf{- 0 . 2 2}$ & $\mathbf{3 3 . 4 7}$ \\
Avg. Bike Stopped Delay (s) & $-\mathbf{2 . 1 1}$ & $\mathbf{7 . 4 6}$ & $\mathbf{1 1 . 7 7}$ \\
Avg. Ped Stopped Delay (s) & $\mathbf{- 1 6 . 8 9}$ & $\mathbf{- 1 2 . 4 6}$ & $\mathbf{- 7 . 6 6}$ \\
Avg. \# of Stops(All) & $\mathbf{1 9 . 4 1}$ & $\mathbf{3 1 . 8 6}$ & $\mathbf{4 3 . 6 0}$ \\
Avg. \# of Auto Stops & $\mathbf{1 8 . 7 4}$ & $\mathbf{3 3 . 6 5}$ & $\mathbf{6 1 . 2 2}$ \\
Avg. \# of HV Stops & $\mathbf{1 3 . 7 2}$ & $\mathbf{4 2 . 4 0}$ & $\mathbf{5 7 . 7 3}$ \\
Avg. \# of Bike Stops & 35.21 & $\mathbf{3 7 . 4 5}$ & $\mathbf{4 0 . 9 5}$ \\
Avg. \# of Ped Stops & $\mathbf{1 7 . 6 9}$ & $\mathbf{1 2 . 8 3}$ & $\mathbf{9 . 5 0}$ \\
Max Queue (ft) & $\mathbf{2 . 9 9}$ & $\mathbf{1 4 . 8 1}$ & $\mathbf{5 7 . 0 9}$ \\
\hline
\end{tabular}

The average delays for pedestrians presented in the above tables include both delays for both mainline pedestrian movements that are on recall as well as the side street pedestrian movements that are actuated. Since the focus of this dissertation is to investigate methods to reduce delay for side street pedestrians, the average delays are analyzed separately for the different auto and pedestrian volume scenarios.

The actuated pedestrian and coordinated through movement delays are shown in Figure 5-15 for the low auto-low ped scenario. For the actuated pedestrian movement, delay reductions range from $51 \%$ at Multnomah and $11^{\text {th }}$ to $67 \%$ at Multnomah and $15^{\text {th }}$, corresponding to a decrease of 17-26 seconds per user depending on the intersection, when the signals are operating free. On the other hand, while the coordinated movement 
delays increase during free operation, the increase is small in magnitude to the order of 12 seconds, which often times is not perceptible for the user.

Similar trends are seen for the low auto-high ped and the high auto-low ped scenarios. For the low auto-high ped scenario, the actuated pedestrian delay reductions range between $25 \%$ - $47 \%$ corresponding to an 11-16 seconds of time savings per user as seen in Figure 5-16 (a). The coordinated movement delay increases range between 3-5 seconds per user as seen in Figure 5-16 (b). The delay reductions for the actuated pedestrian movement fluctuate between $33 \%-45 \%$ corresponding to a 14 second time savings per user for free operation for the high auto-low ped scenario. Figure 5-17(a)-(b) shows the plots for high auto-low ped scenario.

For the high auto-high ped scenario, while the actuated pedestrian movement still experiences reduced delays during free operation, the increase in delay for the coordinated movements is large ranging from $43 \%$ to $171 \%$, which correspond to $12-17$ seconds increase in delay per user as seen in Figure 5-18 (a) - (b). Thus the costs of free operation increases in coordinated movement delays outweigh the benefits of reduced pedestrian delay, when overall delay per person minimization is considered. 


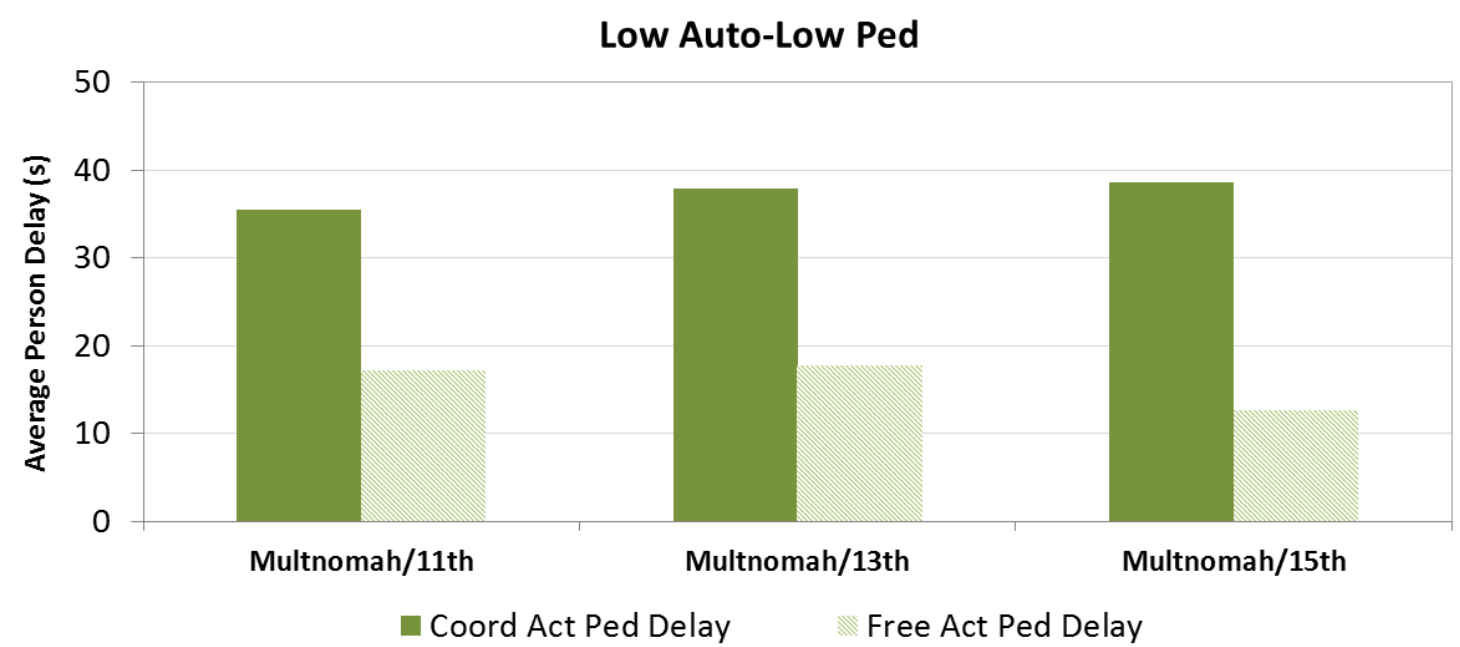

(a)

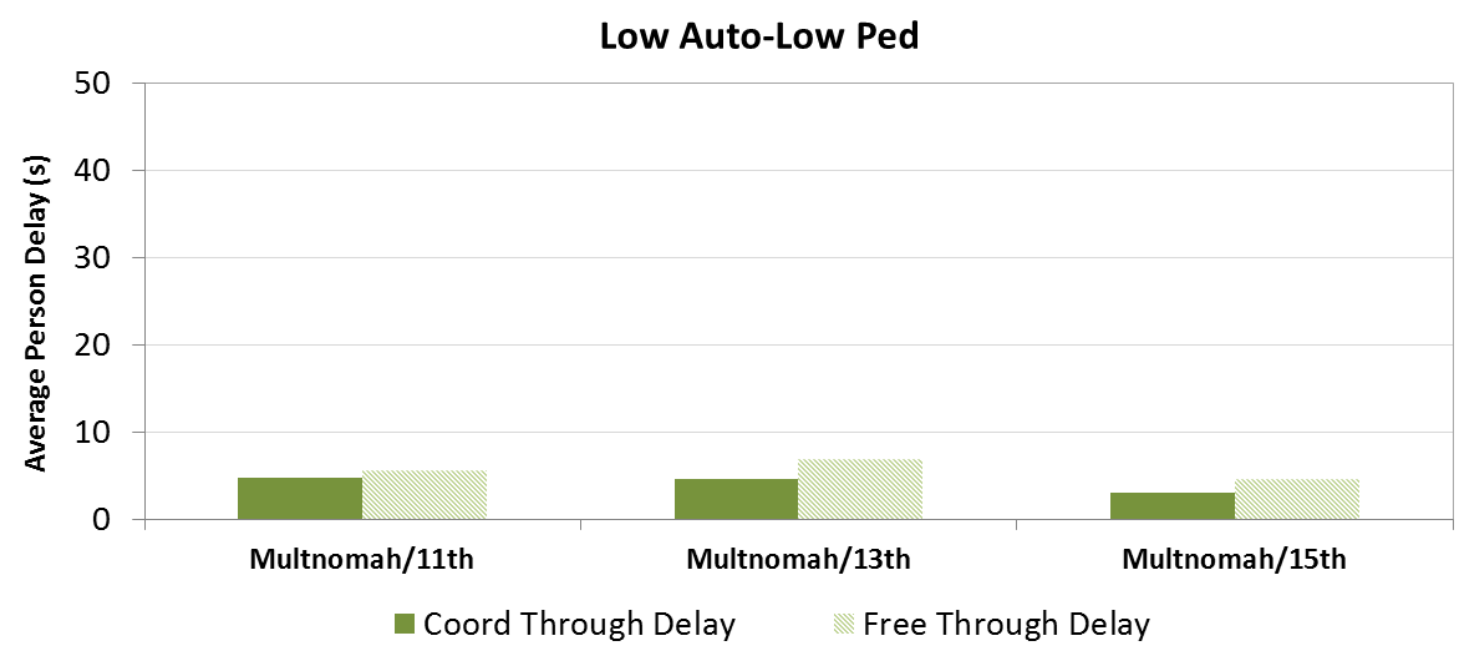

(b)

Figure 5-15 Actuated Pedestrian and Coordinated Movement Delay for Low AutoLow Ped Scenario 


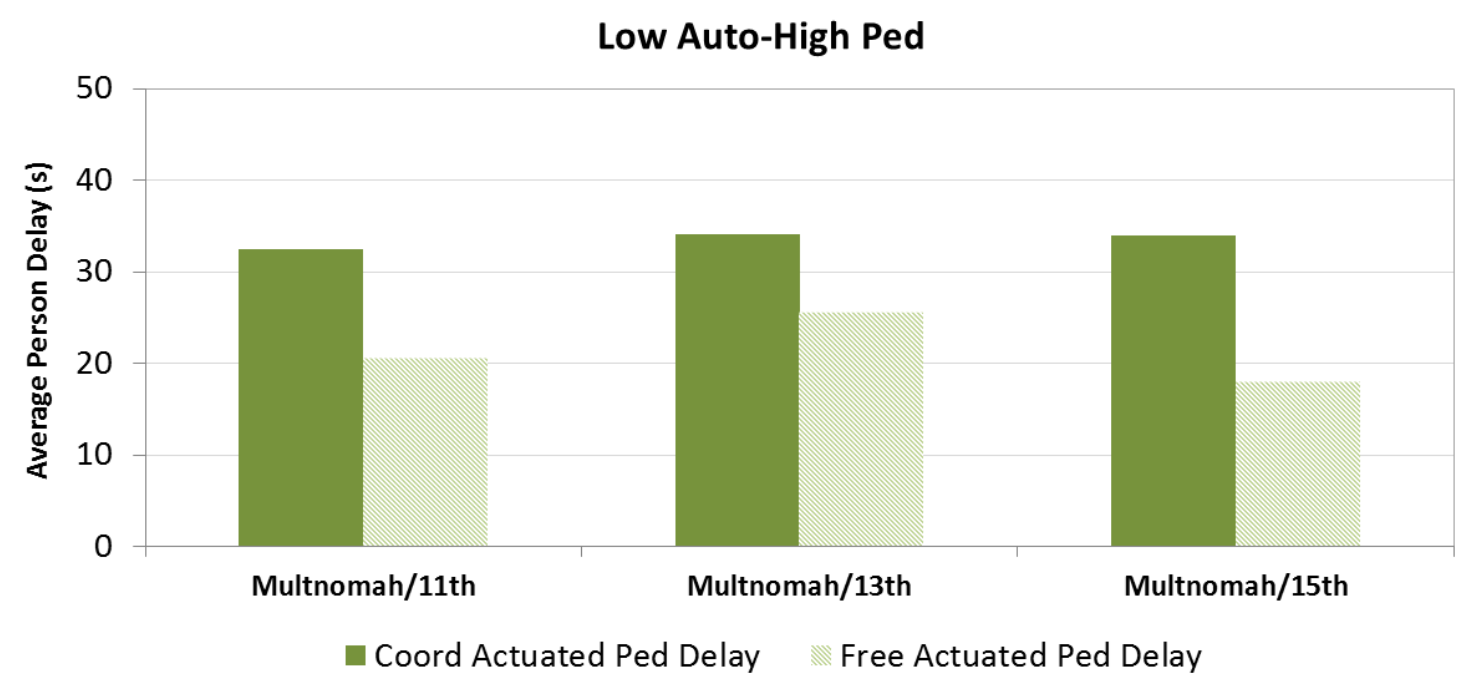

(a)

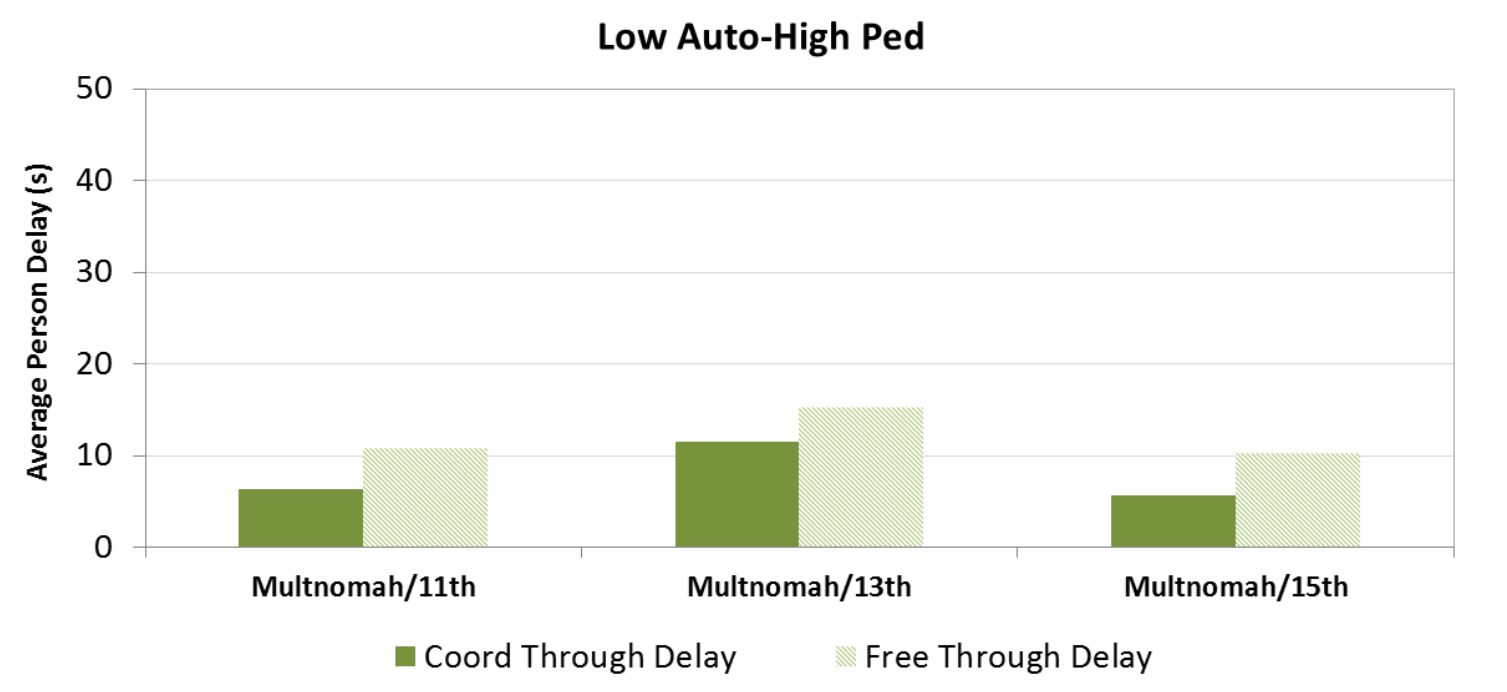

(b)

Figure 5-16 Actuated Pedestrian and Coordinated Movement Delay for Low AutoHigh Ped Scenario 
High Auto-Low Ped

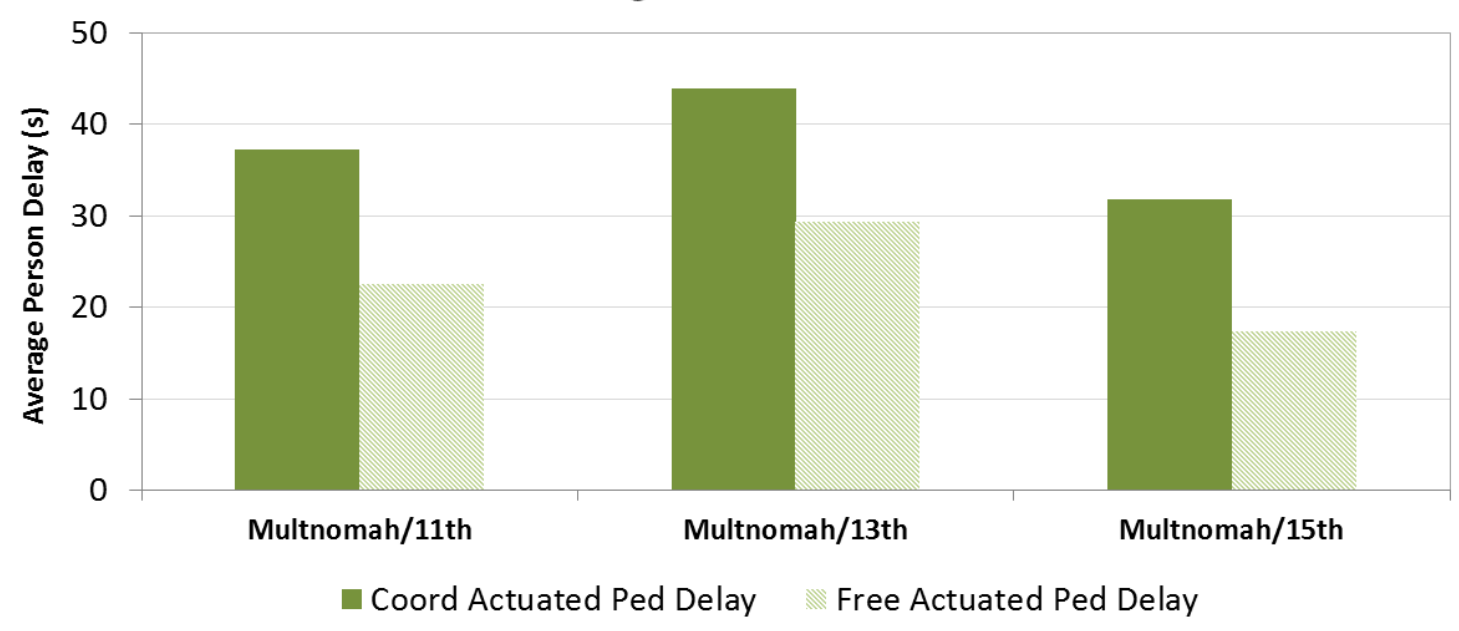

(a)

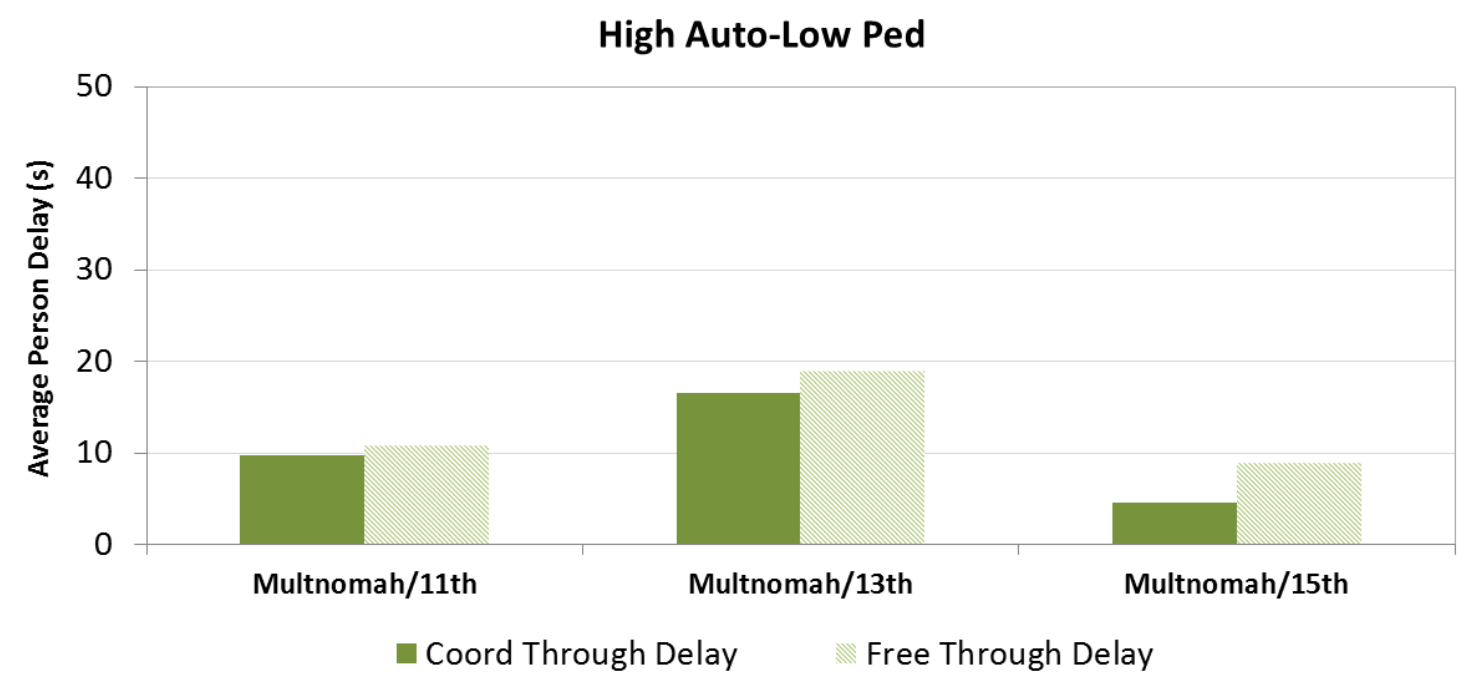

(b)

Figure 5-17 Actuated Pedestrian and Coordinated Movement Delay for the High Auto-Low Ped Scenario 
High Auto-High Ped

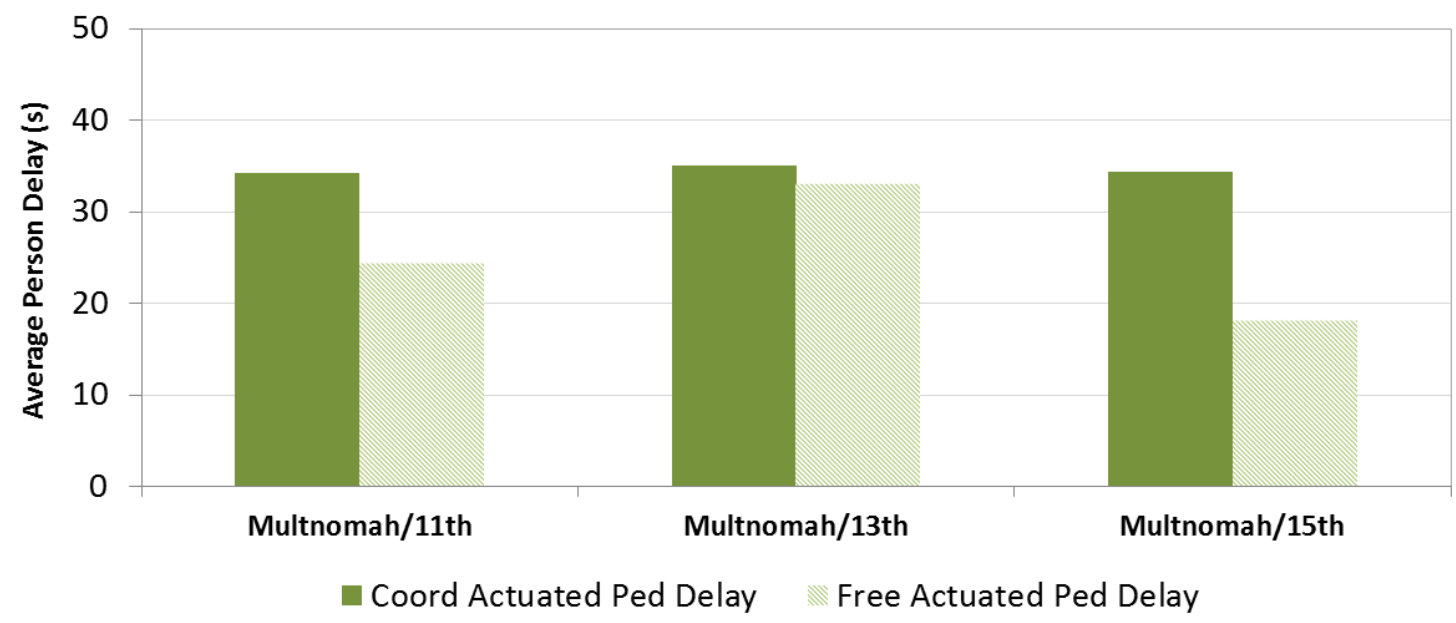

(a)

High Auto-High Ped

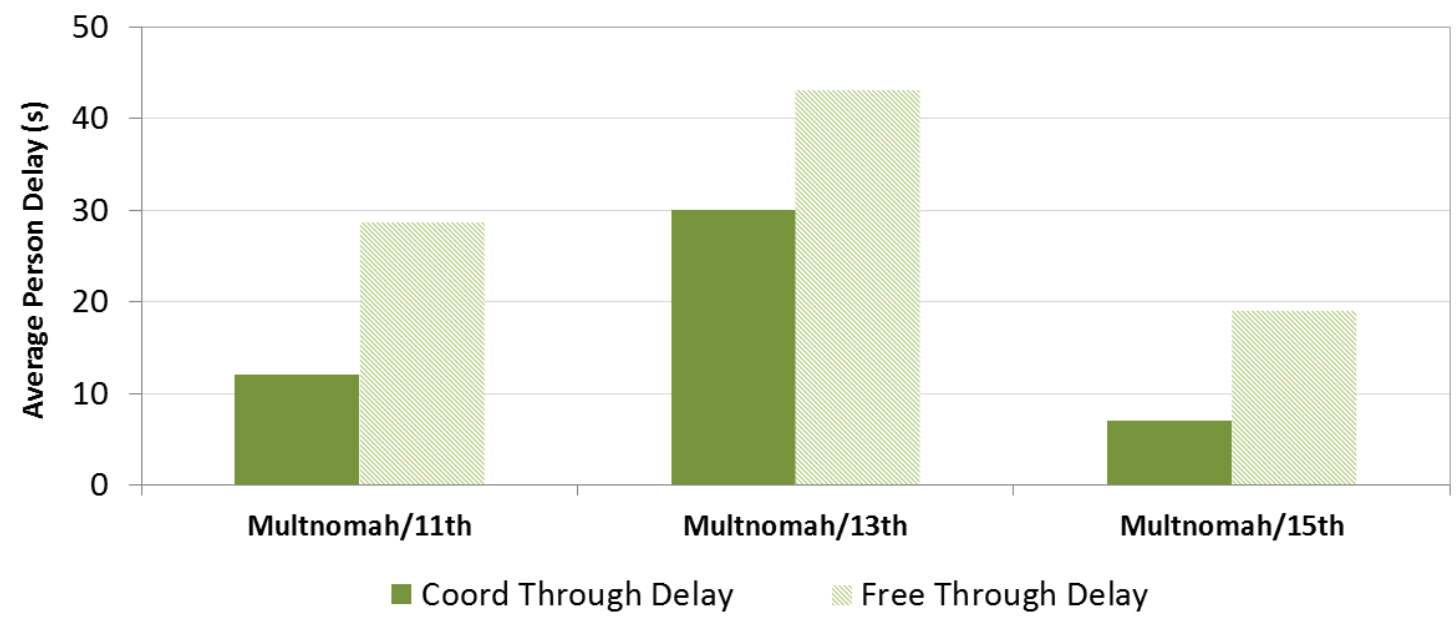

(b)

Figure 5-18 Actuated Pedestrian and Coordinated Through Movement Delays for High Auto-High Ped Scenario 
Combining the overall average delays per user into one graph provides important policy implications, when all users in the network are considered. Figure 5-19 shows the plot of percent change in average delay per user between free and coordinated operation for all 9 scenarios.

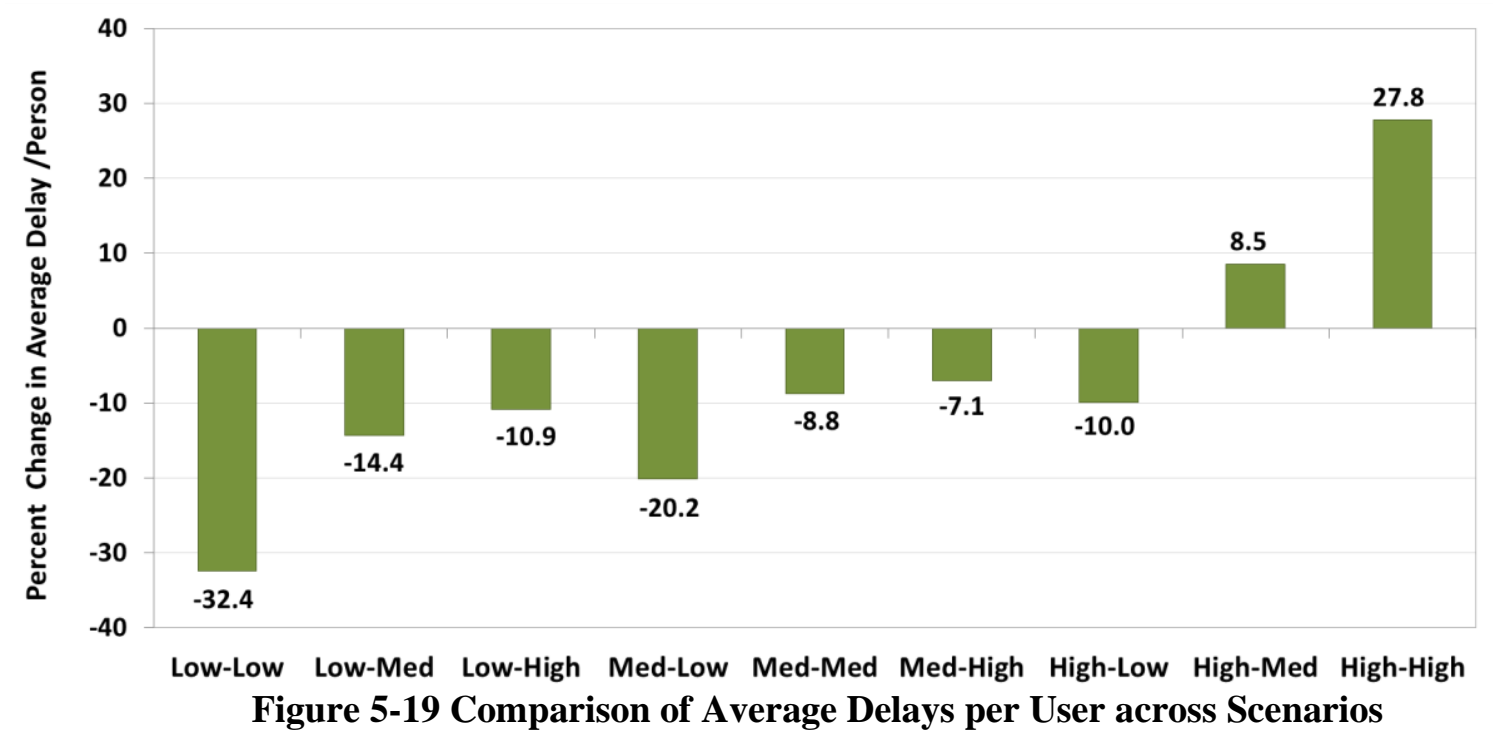

In the above analysis, existing signal timings including cycle lengths, splits and offsets from the field were used for coordinated operation. In order to make a fair comparison between average delays resulting from the two modes of operation, the signal timing optimization software VISTRO was used to optimize the splits and offsets for each scenario, while keeping cycle length constant $(80 \mathrm{sec})$. The resulting overall average delays were compared and are shown in Figure 5-20. The plot shows similar trends as seen earlier in Figure 5-19 with lower average delays observed for free operation for low - medium auto volumes. The average delays along with $p$-values from the $t$-tests are presented in the Appendix. 


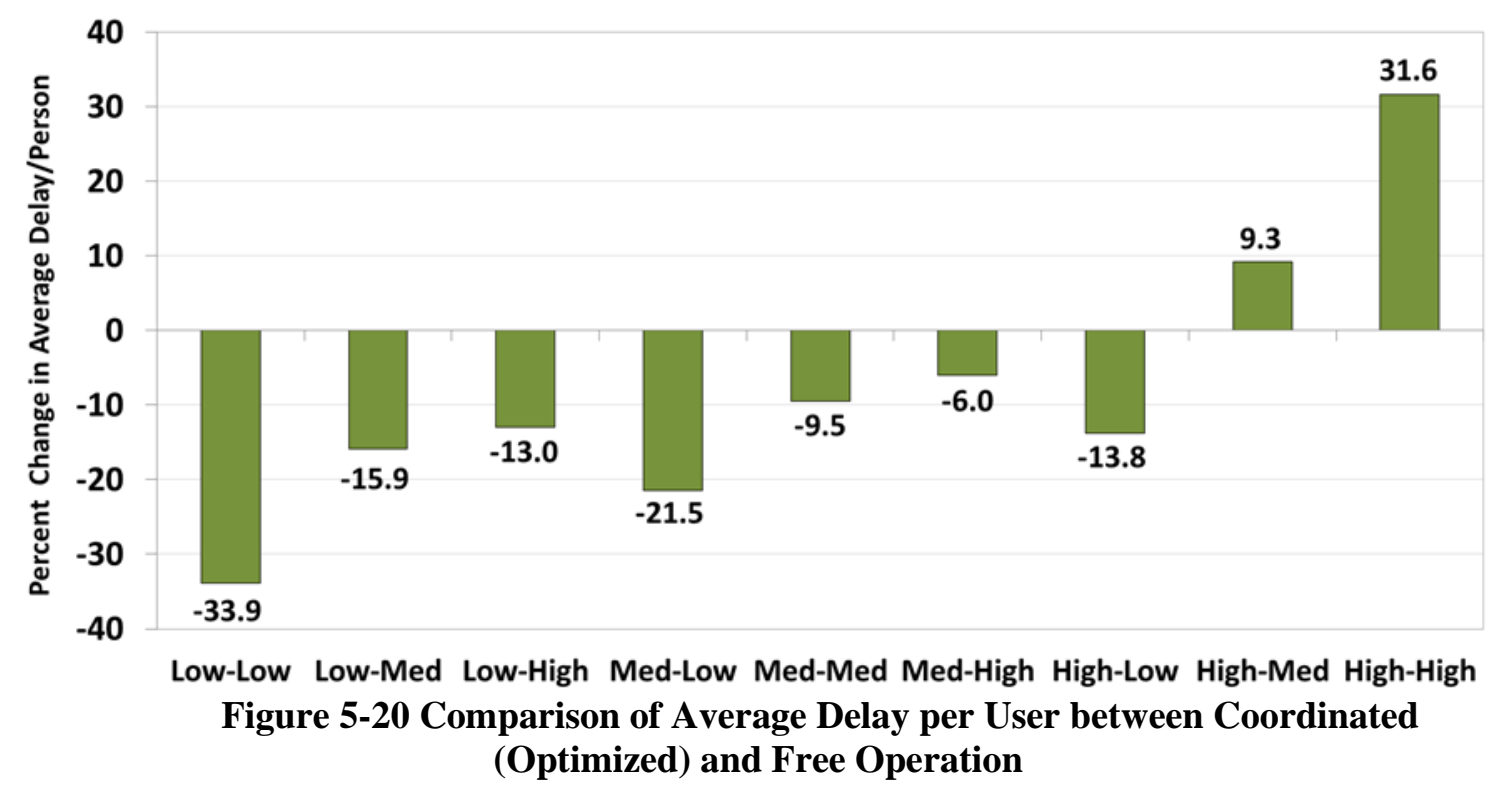

In the analysis presented above, for every tested scenario, pedestrian delay is always lower during free operation irrespective of the auto volumes. However from an overall delay minimization perspective, the above plot clearly indicates that free operation is beneficial for low and medium auto volumes ( $\mathrm{v} / \mathrm{c}$ ratios for mainline through movements $<0.7)$. Coordinated operation is beneficial when auto volumes are nearing capacity (V/C $>0.7)$. Current practice in signal timing at many jurisdictions favors coordination at all times, even during low volume conditions. The analysis presented here has shown that there are clear operational regimes where change in mode of operation has benefits from a network perspective.

\subsubsection{Cycle Length Impacts}

An important consideration in any delay analysis during coordinated operation is the magnitude of cycle length. Previous studies have tested the impact of cycle length on pedestrian delays, with the finding that lower cycle lengths benefit pedestrians (Noland 
1996, Ishaque et al., 2007). In this research, simulations using a higher cycle length of 120 seconds were run to assess whether the finding regarding the feasibility regimes of coordinated and free operation was applicable given the varying vehicular and pedestrian demands. The 120 second cycle length was chosen because it is commonly used in the United States.

The signal timing optimization software VISTRO was used to optimize the splits and offsets for the hypothetical Multnomah Street network using a cycle length of 120s. VISTRO provides two possible optimization levels namely local or network. Optimization at the local level is done on an intersection by intersection basis with no effort towards coordination. Network optimization on the other hand, considers the interaction between signalized intersections (VISTRO User Manual, 2011). The interaction is achieved through a platoon dispersion model. Splits and offsets were optimized along the network using genetic algorithm and the objective of the optimization was to minimize delay and number of stops of the coordinated movements. Four volume combinations of low auto-low ped, low auto-high ped, high auto-low ped and high auto-high ped were simulated and average delay per person was compared between the coordinated and free modes of operation. For each scenario, signal timings were optimized using VISTRO with a set cycle length of 120s. Figure 5-21 shows a plot of the percentage change in average delay comparing the two modes of operation using the higher cycle length of 120 s. The plot follows a similar trend seen earlier in the earlier section, with free operation showing more benefits in terms of overall reduced delay per 
person for low auto volumes and coordination being more beneficial for higher mainline through auto volumes.

Table 5-15 shows the modal differences in average delay per person between free operation and coordinated operation at higher cycle lengths. Similar trends seen earlier are repeated here.

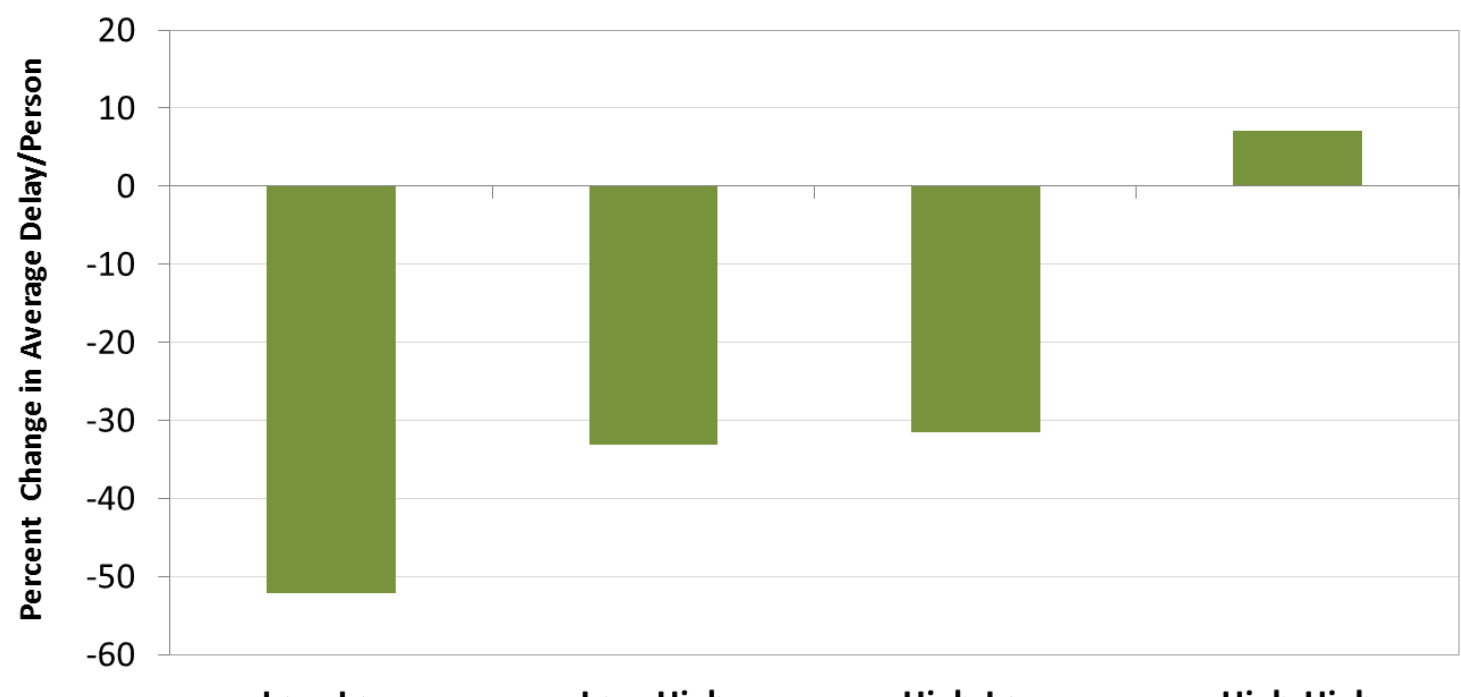

Figure 5-21 Percent Change in Average Delay per Person using 120s Cycle Length

Table 5-15 Average Delay Differences between Modes during Free operation and Coordination using Higher Cycle Lengths

\begin{tabular}{l|rrrrr}
\hline $\begin{array}{c}\text { \% Difference } \\
\text { in Average Delay/person }\end{array}$ & \multicolumn{1}{c}{ All } & \multicolumn{1}{c}{ Auto } & Heavy Veh & \multicolumn{1}{c}{ Bicycle } & \multicolumn{1}{c}{ Pedestrian } \\
\hline Low Auto-Low Ped & -52.10 & -56.76 & -51.13 & 8.52 & -36.19 \\
Low Auto-High Ped & -33.11 & -28.57 & -19.91 & 103.83 & -37.40 \\
High Auto-Low Ped & -31.54 & -34.06 & -32.13 & 40.83 & -38.64 \\
High Auto-High Ped & 7.08 & 23.66 & 31.11 & 53.93 & -28.10 \\
\hline
\end{tabular}

Free operation benefits pedestrians with overall delay being lower during all analyzed scenarios. Bicycle delay is higher because the lack of coordination during free operation implies that bicyclists have to stop more often at intersections, thereby increasing their delay. Auto and heavy vehicle delay is lower during free operation for lower auto 
volumes and for the high auto-low ped scenario. At high auto volume and medium to high pedestrian demand, coordinated delay is lower than free delay for autos and heavy vehicles.

A comparison of the average delays across cycle lengths and during the free operation is also performed. Except for bicycles, overall average delay as well as delay for other modes increased with higher cycle length. Two plots for the low auto-low ped and the high auto-high ped scenarios comparing average delays across modes between the two cycle lengths and free operation are shown in Figure 5-22. The plots for the other scenarios follow similar trends.

The implication from this analysis is that longer cycle lengths while in coordination primarily benefit the coordinated through movements (auto, heavy vehicle bicycle and main street pedestrian if rest-in walk is enabled) while increasing delay for all other movements. Due to the increase in side street vehicle and non-coordinated movement delay as well as side street pedestrian delay, overall delay increases for all modes except bicycle due to the higher cycle length. 


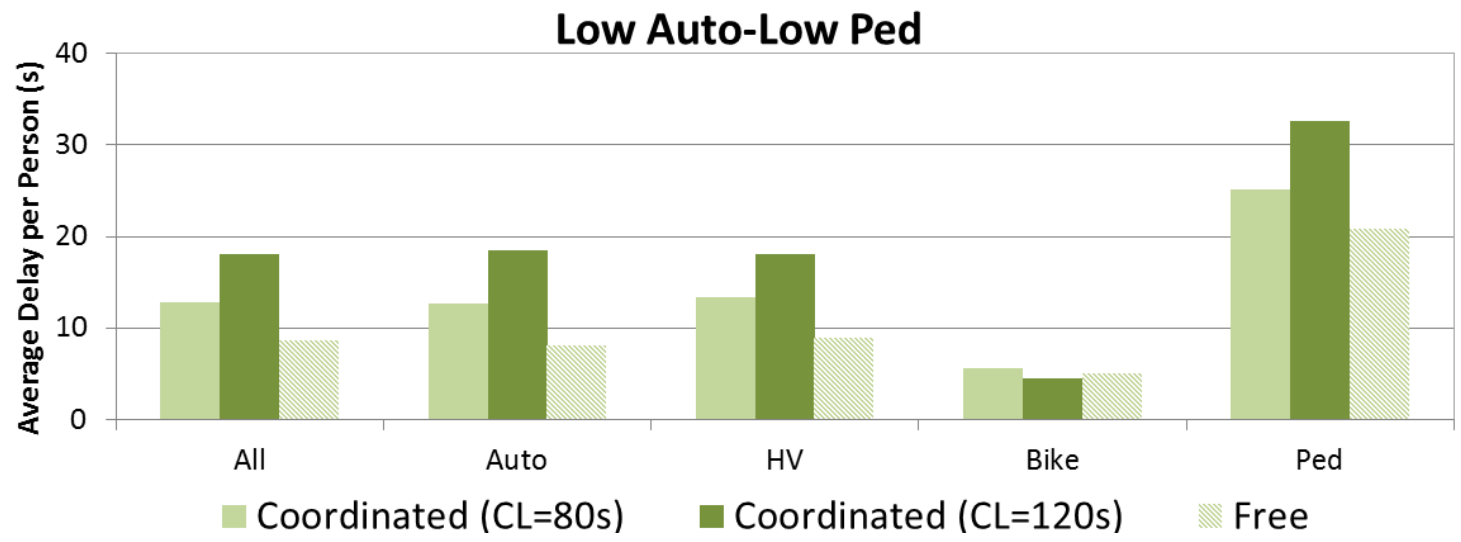

(a)

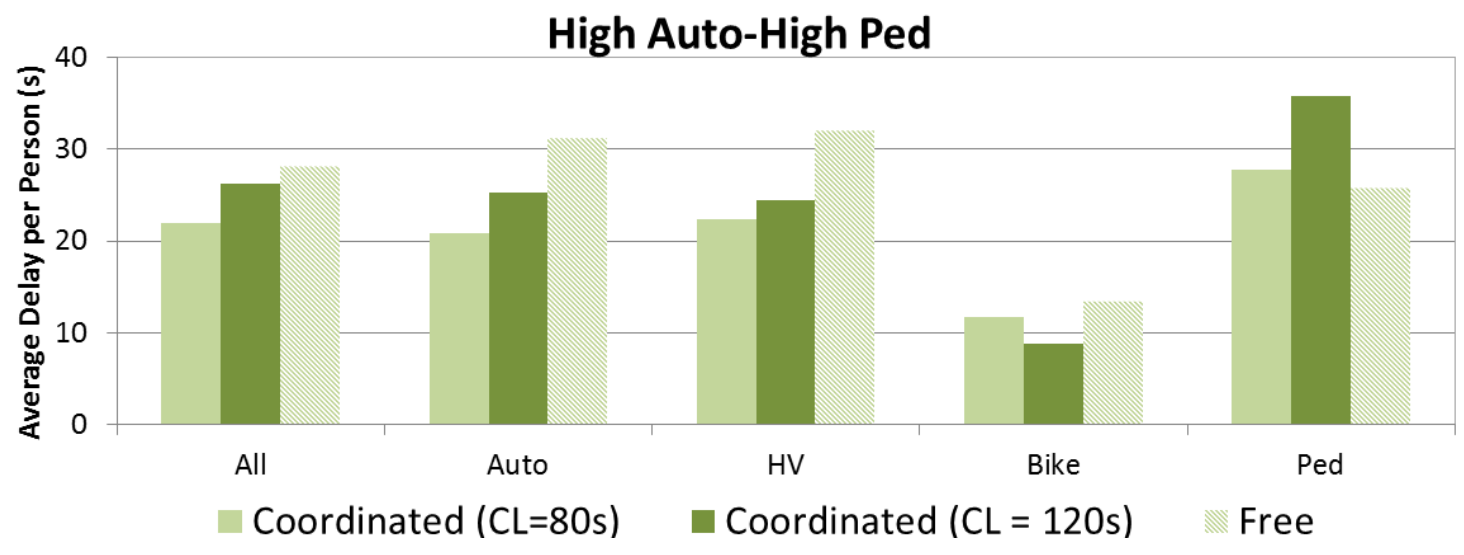

(b)

Figure 5-22 Comparison of Modal Delays across Cycle Lengths and Free Operation

\subsubsection{Detection on all Approaches}

The analysis in previous sections assumed detection only for the side street movements (vehicle and pedestrian). The major street through movements did not have any detection in the prior analysis during coordination and during free operation pedestrian recall was active for the mainline pedestrian movements, which in turn ensured that the vehicular 
green for the main line through movements was also served. In this section, the impacts of detection on all approaches are studied.

The simulation analysis conducted in the prior section was repeated with the addition of detection to the major street through lanes and removal of pedestrian recall for the major street pedestrian phases. The objective of this exercise was to determine if the additional information provided to the signal controller via the detectors, increased signal responsiveness and induced further delay reductions. Average delay per user during coordination $(\mathrm{CL}=80 \mathrm{sec})$ was compared to delay resulting from fully actuated free operation and is shown in Figure 5-23. Overall trends were similar to the prior analysis. The biggest benefit of fully actuated free operation is seen for the low auto-low ped scenario with overall reduction of $47 \%$. For the high auto-high ped scenario, the overall delay reduction was negligible and the trend indicated that the benefits of coordination would outweigh those of free operation for any further increase in demand.

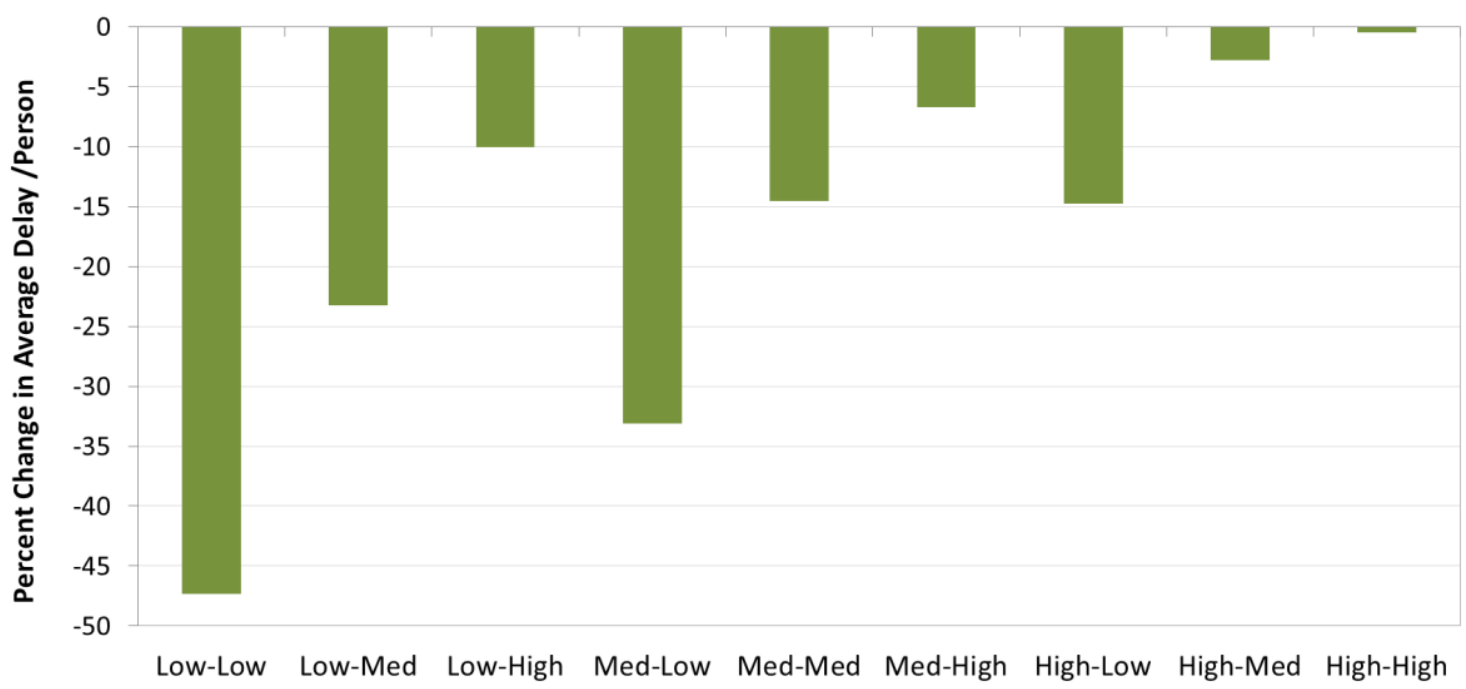

Figure 5-23 Comparison of Average Delays during Coordination and Fully Actuated Free Operation 
Table 5-16 shows the percent difference in average delay between free and fully actuated free operation. The percent difference is calculated using the formula:

$$
\% \text { Difference }=\frac{(\text { Fully Act Free Delay-Free Delay }) * 100}{\text { Free Delay }}
$$

Negative values imply that the delay during full actuation is less than delay when it is semi-actuated. As the values indicate, there are benefits to full actuation in terms of reduced overall average delay especially during low auto-low ped scenario. While the high auto-high ped scenario also shows reduced delays during free operation when it is fully actuated, the resulting average delay is not different from the delay resulting from coordinated operation. At $\mathrm{V} / \mathrm{C}$ ratios greater than 0.7 , coordinated operations are recommended and as such detection on all approaches during coordination may not be necessary unless fully actuated coordination is considered. The premise of fully actuated coordination is to allow a portion of the coordinated phases to be actuated so that they can gap out and allow other phases to be served when demand is low (Day, 2007). Benefits via reductions in $\mathrm{v} / \mathrm{c}$ ratios and fewer occurrences of split failures were observed (Day, 2007).Therefore, fully actuated free operation has most benefits during the low volume scenarios, by allowing the signal to be more responsive to light traffic conditions. 
Table 5-16 Differences in Delay between Free and Fully Actuated Free Operation

\begin{tabular}{l|r}
\hline Scenario & \multicolumn{2}{|c}{$\begin{array}{c}\text { Difference in \% } \\
\text { Average }\end{array}$} \\
& Delay \\
\hline Low Auto-Low Ped & -22.08 \\
Low Auto-Med Ped & -10.36 \\
Low Auto-High Ped & 0.90 \\
Med Auto-Low Ped & -16.23 \\
Med Auto-Med Ped & -6.32 \\
Med Auto-High Ped & 0.32 \\
High Auto-Low Ped & -5.36 \\
High Auto-Med Ped & -10.45 \\
High Auto-High Ped & -22.08 \\
\hline
\end{tabular}

\subsubsection{Discussion}

This research has demonstrated empirically, the various traffic regimes that are best suited for strategies that benefit pedestrians from an efficiency perspective. The strategies tested in the simulation included changes to signal controller mode of operation, cycle length variations and fully actuated signal operation.

Under the conditions assumed in the simulation, the results demonstrate that free operation is always beneficial for pedestrians leading to reduced delay as compared to coordinated operation. Free operation also shows network benefits under low and medium volumes $(\mathrm{V} / \mathrm{C}$ for main line through movements $<0.7)$. Detection on all approaches was shown to further increase the efficiency of free operation at low volumes by inducing further reductions in average delay. Coordinated operation was beneficial when traffic volumes are high $(\mathrm{V} / \mathrm{C}>0.7)$. While in coordination, lower cycle lengths generally benefit pedestrians. 
System operators and signal timing engineers face tradeoffs each day while operating the signals at various intersections. The primary tradeoff is balancing safety vs. efficiency. Secondary tradeoffs include balancing the delay between modes such that no mode is unduly penalized. Although traditional signal timing policies have favored motor vehicles, this policy needs to be reconsidered if cities want to develop livable communities that promote walking and bicycling. Therefore, with the aim of providing guidance to system operators, a concept graphic based on the results obtained in this research has been developed, that seeks to consolidate the findings with the objective of informing policy decisions. Figure 5-24 shows the graphic with recommendations for strategies for operational decisions at the intersection level.

Although the results showed that free operation provides benefits when $\mathrm{V} / \mathrm{C}$ is less than 0.7 for the main line through movements, this graphic takes a conservative approach by recommending free operation when $\mathrm{V} / \mathrm{C}$ is less than 0.5 . Coordination with the ability to manage the type of pedestrian response is recommended for the middle regime where $\mathrm{V} / \mathrm{C}$ is between 0.5 and 0.8 and the pedestrian actuation frequency is low or medium. The type of response for pedestrian service will depend on the policies adopted by agencies and the priority hierarchy assigned for each mode. Pedestrian friendly strategies such as temporary removal of a signal from coordination by increasing permissive length or providing pedestrian priority service could be employed if the agencies want to prioritize pedestrians. For $\mathrm{V} / \mathrm{C}$ ratios greater than 0.8 , coordination is recommended with short cycle lengths, so that pedestrians are not faced with large delays. It is recommended that pedestrian signals be actuated with pushbuttons when the 
actuation frequency is less than $70 \%$ of the number of cycles during the design time period. Higher actuation frequencies indicated the need for pedestrian recall. Placing the pedestrian movements on recall during periods of low actuation have the potential to impose unwarranted delays on the main street movements leading to larger delay overall and lower system efficiency.

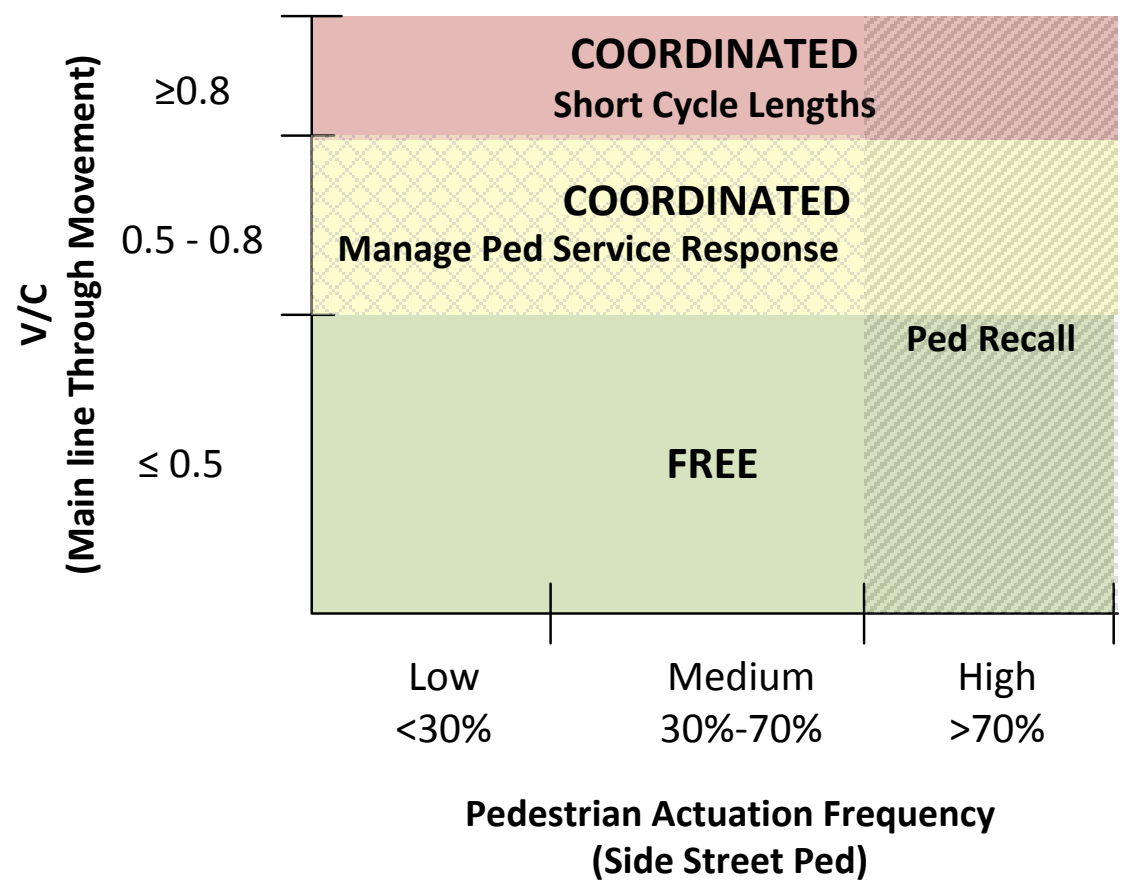

Figure 5-24 Strategy for Changing Signal Controller Mode of Operation

Switching the pedestrian operation from actuated to recall based on time of day could be challenging for pedestrians given the existing detection technology limitations at intersections. In the future, this could be mitigated with advances in automated detection technology that could respond efficiently to fluctuations in pedestrian volume.

To implement the strategies discussed above using the graphic, the primary inputs required are major street through volumes and side street pedestrian actuation frequency. Typically, many cities gather volumes on arterials using some form of detection (loop, 
microwave, radar or video). The actuation frequency is easily logged through the signal controller.

Using time of day auto volumes and pedestrian actuation patterns, system operators can identify time periods during the day when a certain signal controller mode of operation is justified based on the lowest overall average delay per user.

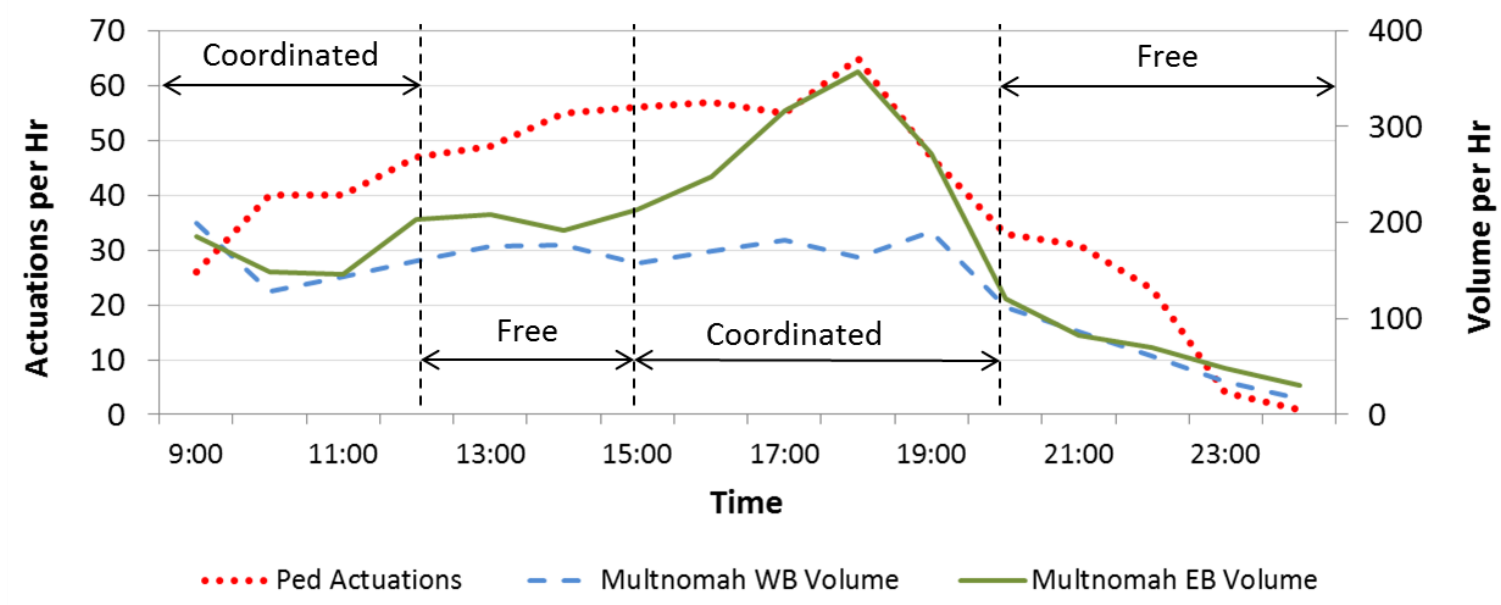

Figure 5-25 Concept Mode of Operation based on Time of Day

As an example, Figure 5-25 shows the auto volumes and pedestrian actuations for the Multnomah Corridor on Thursday, October 3, 2013. As discussed earlier, the pedestrian volumes along the three analyzed intersections at this corridor are higher during mid-day and PM peak periods compared to the AM peak. Therefore, the corridor could stay in coordination during the AM peak hour until noon (6 AM - $12 \mathrm{PM})$ to prioritize auto, bicycle and transit volumes along the major street. This time period would probably represent the high auto scenario where coordinated operation is recommended as discussed earlier. Due to the higher pedestrian demand and low vehicle demand during noon and early afternoon, the signals could switch to coordinated operation between 12 - 
3 PM to benefit pedestrians (Low Auto - High Ped). Between 3 - 8 PM, the signals could switch back to coordinated operation to benefit the heavy PM peak period traffic volumes (High Auto - High Ped). Allowing the signals to operate in a free mode at night (after 8 PM) would allow the signals to be more responsive to the low traffic conditions (Low Auto - Low Ped).

Allowing free operation during certain times of the day could be easily applicable to signals that are close to high pedestrian demand generators such as shopping malls and theaters. The strategy could also be used at signals with low compliance rate or high pedestrian crash rate to improve conditions. While this research presented an empirical framework to assess the optimal mode of operation based on overall average delay per user, there are other factors that could also predispose certain locations to one mode of operation. Closely spaced intersections (1/4 mile or less) can benefit from coordination due to platooning effect (NACTO, 2014). The ratio of side street to major street volume is another factor that could impact the decision on mode of operation. If the ratio is low, coordinated operation may be preferred as it would benefit the higher volumes on the major street. Conversely, higher ratios would favor free operation. This strategy of changing the signal controller mode of operation would be best suited for minor arterials with intersecting cross streets that have low vehicular volumes, but may have moderate or high pedestrian demand.

The framework designed here is applied to another corridor in Portland, Oregon to assess the transferability of the findings. The analysis is repeated for Division Street 
which is a major arterial corridor and the findings and discussion are presented in the following section.

\subsection{Case Study}

Division Street is a major east-west arterial corridor in Portland, Oregon carrying approximately 18,000 vehicle trips per day. Three intersections were chosen for simulation analysis to test the robustness of the operational strategy described in the previous section. The intersections chosen were SE $119^{\text {th }}$ Avenue, SE $122^{\text {nd }}$ Avenue and SE $130^{\text {th }}$ Avenue along SE Division Street. Along this stretch, Division Street has two lanes in each direction, with additional turn lanes at intersections. SE $119^{\text {th }}$ Avenue and SE Division Street is a T-intersection, with one travel lane on SE $119^{\text {th }}$ Ave. SE $122^{\text {nd }}$ Avenue has two through lanes and additional right and left turn lanes in the north and south bound directions. SE $130^{\text {th }}$ Avenue had one traffic lane in each direction. This stretch of the corridor was chosen because the intersection of SE $122^{\text {nd }}$ Avenue and SE Division Street is a test bed intersection for the Portland Bureau of Transportation's Signal and Street Lighting Division for evaluating new technologies. This intersection was equipped with a variety of detection technologies and afforded the possibility of field deployment of the strategies developed here. In addition, the intersection of SE $122^{\text {nd }}$ and SE Division St was identified as a high crash location in Portland, Oregon (Portland Bureau of Transportation, 2012). A total of 64 crashes involving pedestrians were reported between 2000-2009 along SE $122^{\text {nd }}$ Avenue, with over half of them occurring at 
signalized intersections (PBOT, 2012). Figure 5-26 shows the network of three intersections that were included in the simulation.

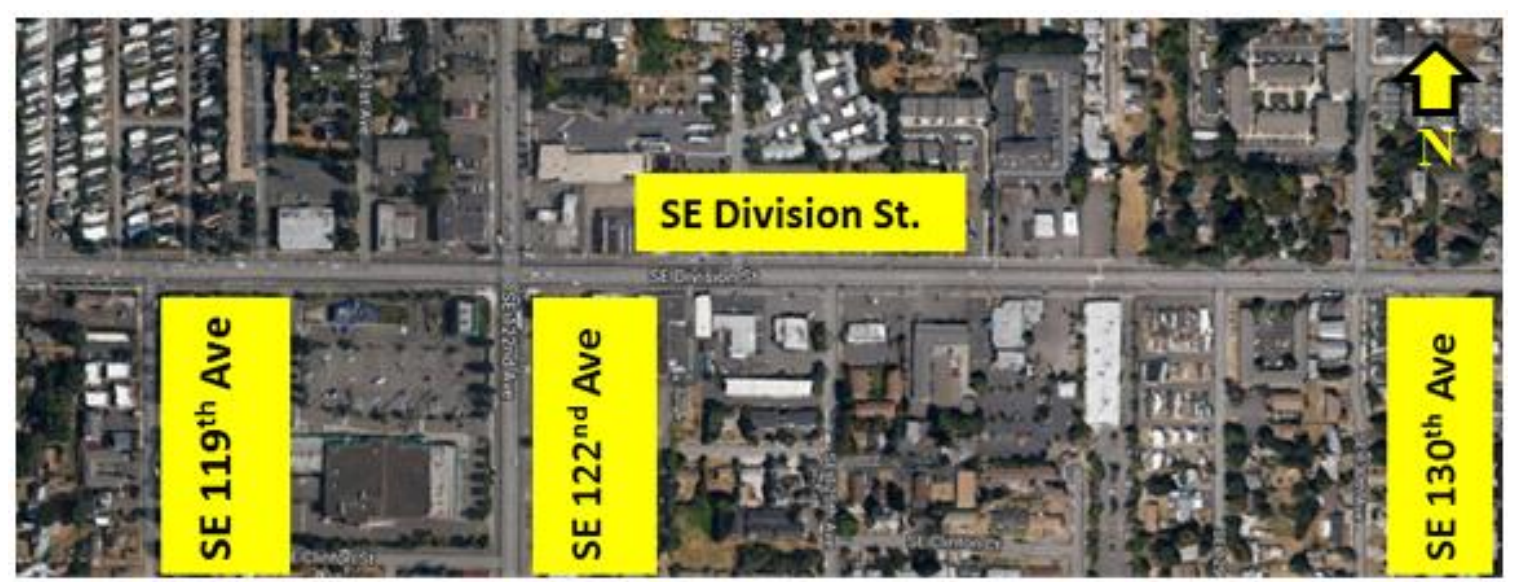

Figure 5-26 Division Street Corridor

\subsubsection{Data Inputs for Simulation}

The data inputs for simulation were gathered from a variety of sources. The lane configuration and network geometry was derived from Google Earth satellite imagery. A background picture of the network was saved and imported into VISSIM for network coding and development. The width of all lanes including turn lanes was assumed to be $10 \mathrm{ft}$. All pedestrian crosswalk widths were assumed as $6 \mathrm{ft}$. The speed on SE Division Street as well as SE $122^{\text {nd }}$ Avenue was assumed as $35 \mathrm{mph}$. The speeds on SE $119^{\text {th }}$ Avenue and SE $130^{\text {th }}$ Avenue were assumed as $25 \mathrm{mph}$. The speeds were either obtained from posted speed limit signs on the respective facilities or assumed when the posted speed limits were not available. Pedestrian speeds were allowed to vary between $3-5$ mph. The traffic compositions on the major and minor streets were assumed as $95 \%$ cars and $5 \%$ heavy vehicles. To simplify the analysis bicycles were not included in the network. A coded VISSIM network is shown in Figure 5-27. 


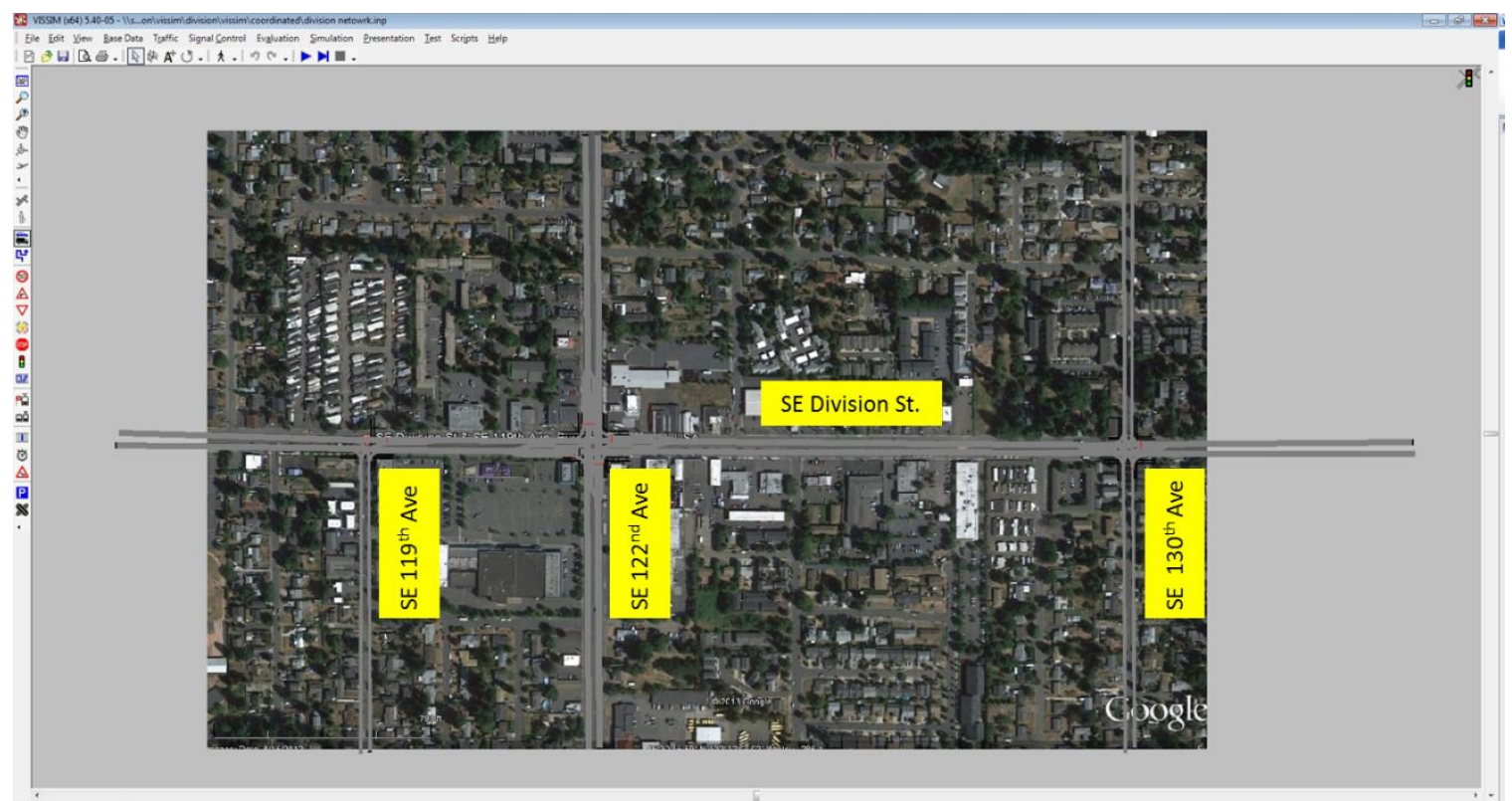

Figure 5-27 Division Street Network in VISSIM

Traffic counts for simulation were obtained from the City of Portland for SE $115^{\text {th }}$ Avenue and SE $130^{\text {th }}$ Avenue. Counts were available for the 7-9 AM and 4-6 PM peak hour time periods. Detailed turning movement counts were available for all approaches at these intersections. Although these counts were taken in 2010-2011, they were used in the analysis due to lack of other count data. Since the geometry of SE $115^{\text {th }}$ Avenue was similar to SE $119^{\text {th }}$ Avenue, the counts taken on $115^{\text {th }}$ Avenue were assumed for SE $119^{\text {th }}$ Avenue. Using the volumes from the City of Portland, the $\mathrm{V} / \mathrm{C}$ ratios for mainline Division Street at $119^{\text {th }}$ Ave and $130^{\text {th }}$ Ave using PM peak hour counts were estimated in the range of $0.4-0.7$. Using the earlier definitions of $\mathrm{V} / \mathrm{C}$ ratios, these numbers were in the medium volume range. Therefore volumes for $122^{\text {nd }}$ Ave were also chosen such the $\mathrm{V} / \mathrm{C}$ ratios were in the medium range of $0.4-0.7$. Based on the geometry of the intersection at $122^{\text {nd }}$ Avenue and Division Street which included two through lanes, the volumes on SE $122^{\text {nd }}$ Avenue were primarily assumed to be through movements with 
minimal turning volume. The counts from 5-6 PM were used for analysis and are shown in Figure 5-28. Pedestrian counts were available for some crosswalks and these counts were in the low pedestrian volume range. For crosswalks where counts were unavailable, counts were assumed such that they were in the low range as well.

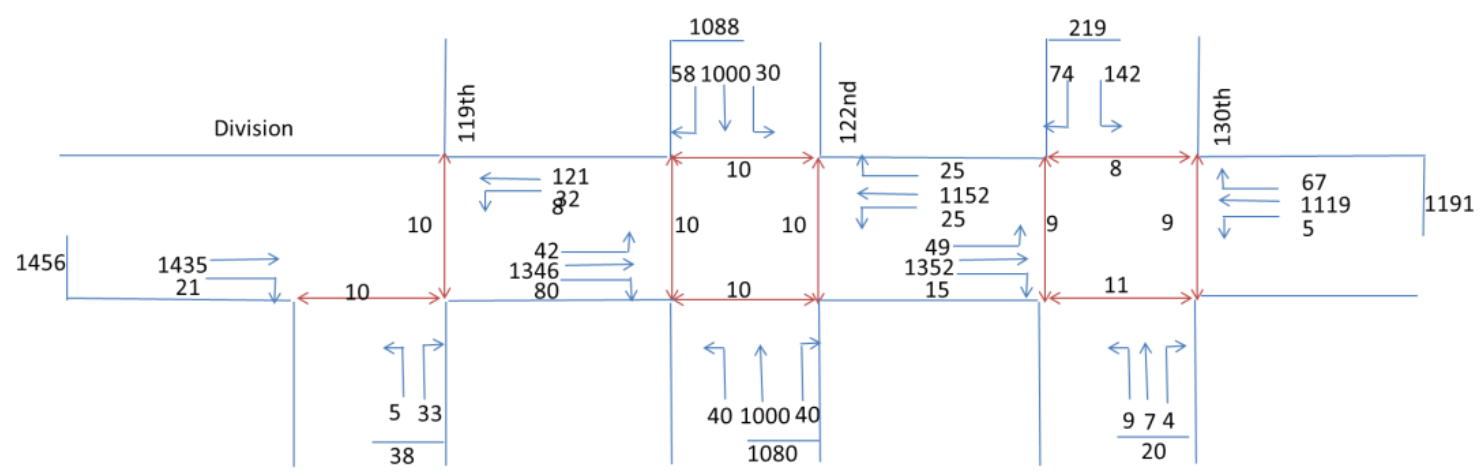

Figure 5-28 Division Street Network Volumes

Signal timings and phasing sequences for the RBC controller were obtained from the City of Portland's timing plans. The intersections of SE $119^{\text {th }}$ Avenue and SE $130^{\text {th }}$ Avenue were equipped with the older Type 170 signal controllers and were coordinated whereas the $122^{\text {nd }}$ Avenue intersection was operating in free mode. Two cycle lengths of 110 and 120s corresponding to three time of day plans were utilized at the coordinated intersections. The 120s cycle length was used between 2:30 and 6:30 PM and as such encompassed the PM peak period. Since the objective of this analysis was to evaluate the feasibility regimes for coordinated and free operation and to quantify the benefits for either operation, the three section network was placed in coordination during the simulation with a cycle length of $120 \mathrm{sec}$. Splits and offsets for the $120 \mathrm{~s}$ cycle length at each intersection were optimized using VISTRO with the objective of minimizing delay and stops for the coordinated movements as shown in Figure 5-29. 


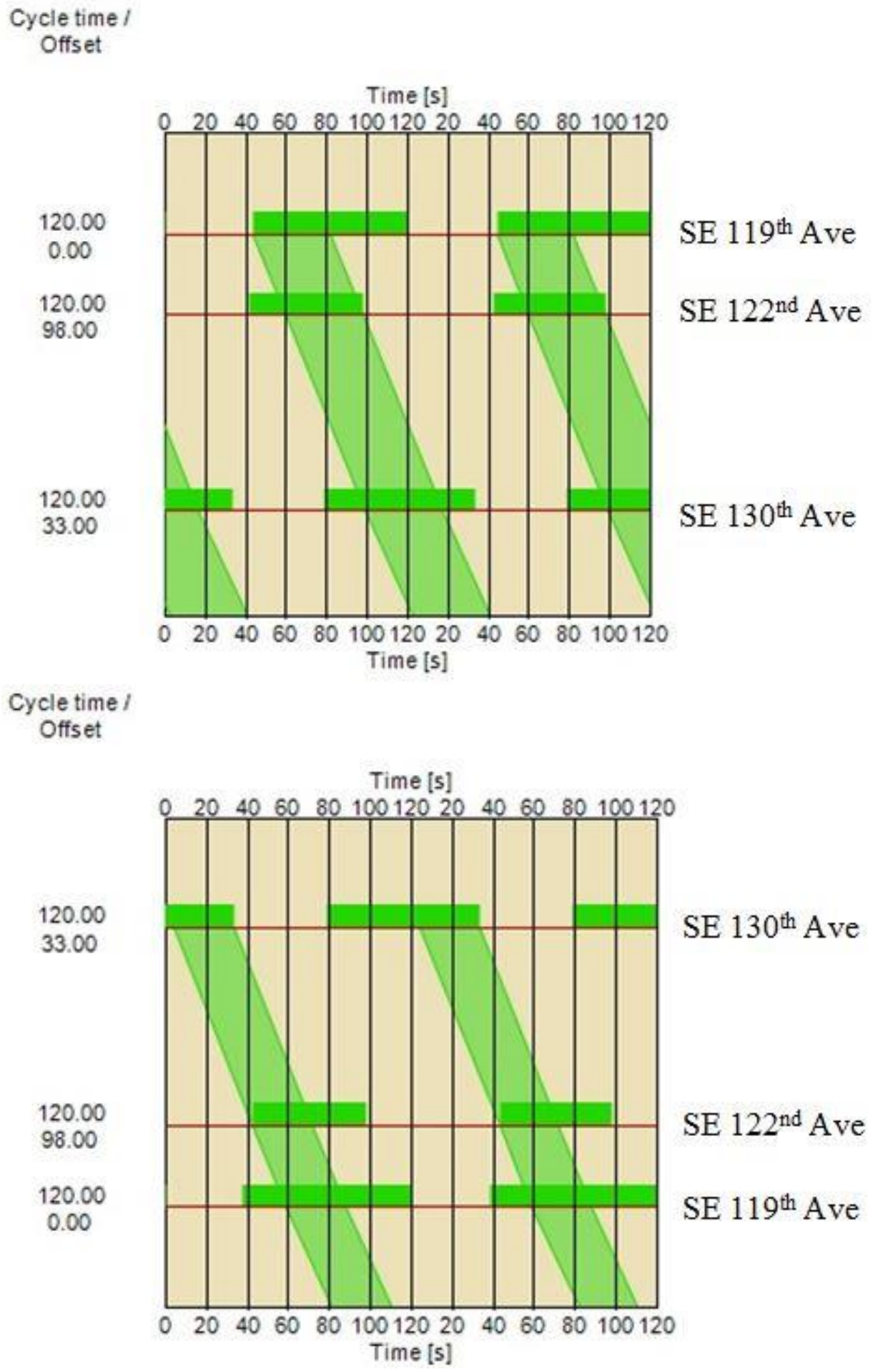

Figure 5-29 East and Westbound Bandwidths for Progression on Division Street 


\subsubsection{Results}

Based on the prior findings on the Multnomah corridor, the expected finding was that overall average delay for the medium auto-low pedestrian scenario compared between the coordinated and free operation would be lower for the free operation. 10 simulation runs were performed for the coordinated and free operations (fully actuated) and average delay metrics were extracted. As expected, the overall average delay was lower in the free mode of operation. Average delays for all modes (cars, heavy vehicles and pedestrians) were also lower in this scenario. Figure 5-30 shows the plot of percent change in average delays overall and by mode between the coordinated and free modes of operation.

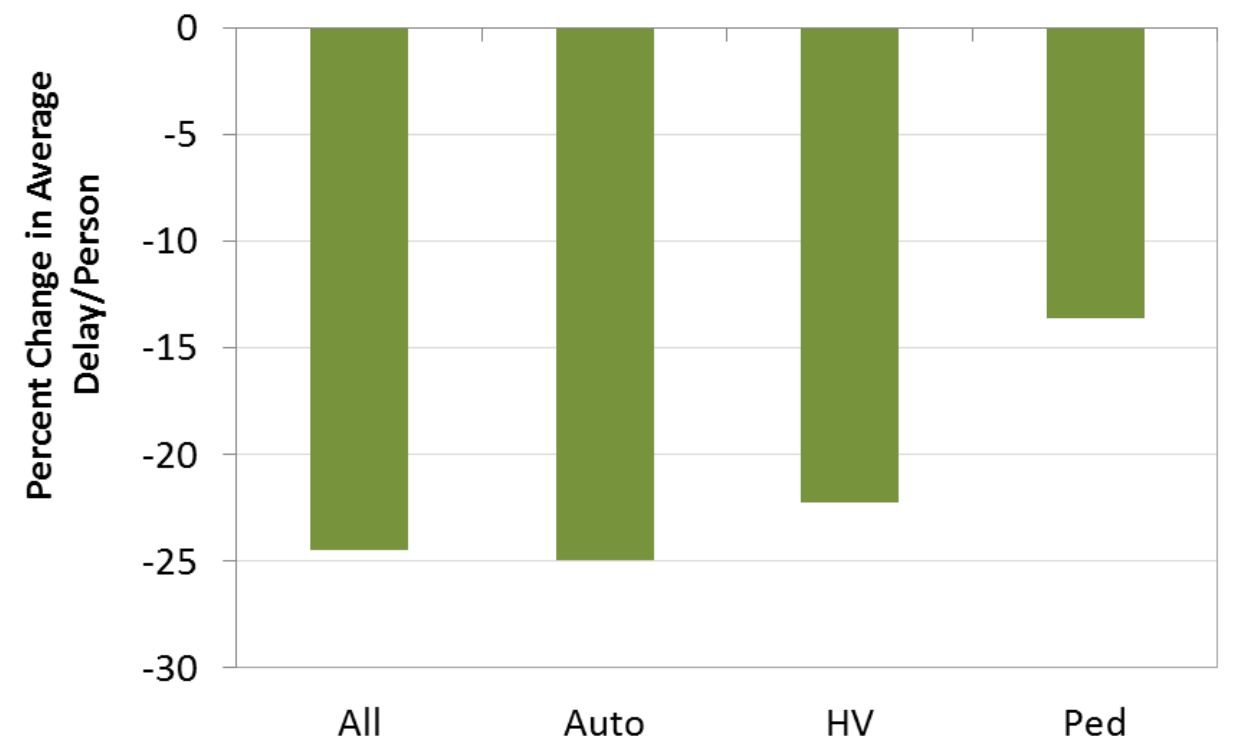

Figure 5-30 Average Delay Comparison between Coordinated and Fully Actuated Free Modes on Division Street Network

As outlined earlier, pedestrian and auto volumes were chosen for the low auto - low ped and the high auto - high ped scenarios based on $\mathrm{V} / \mathrm{C}$ ratio ranges and pedestrian phase frequency. The analysis presented earlier for hypothetical Multnomah Street network was 
repeated for just the low auto-low ped and high auto-high ped scenarios along the Division Street corridor as the objective was to assess whether general trends seen earlier were followed. Figure 5-31 shows the difference in percent average delays on Division Street for the two scenarios. Note that the medium auto-low ped scenario corresponded to the existing conditions as described in earlier.

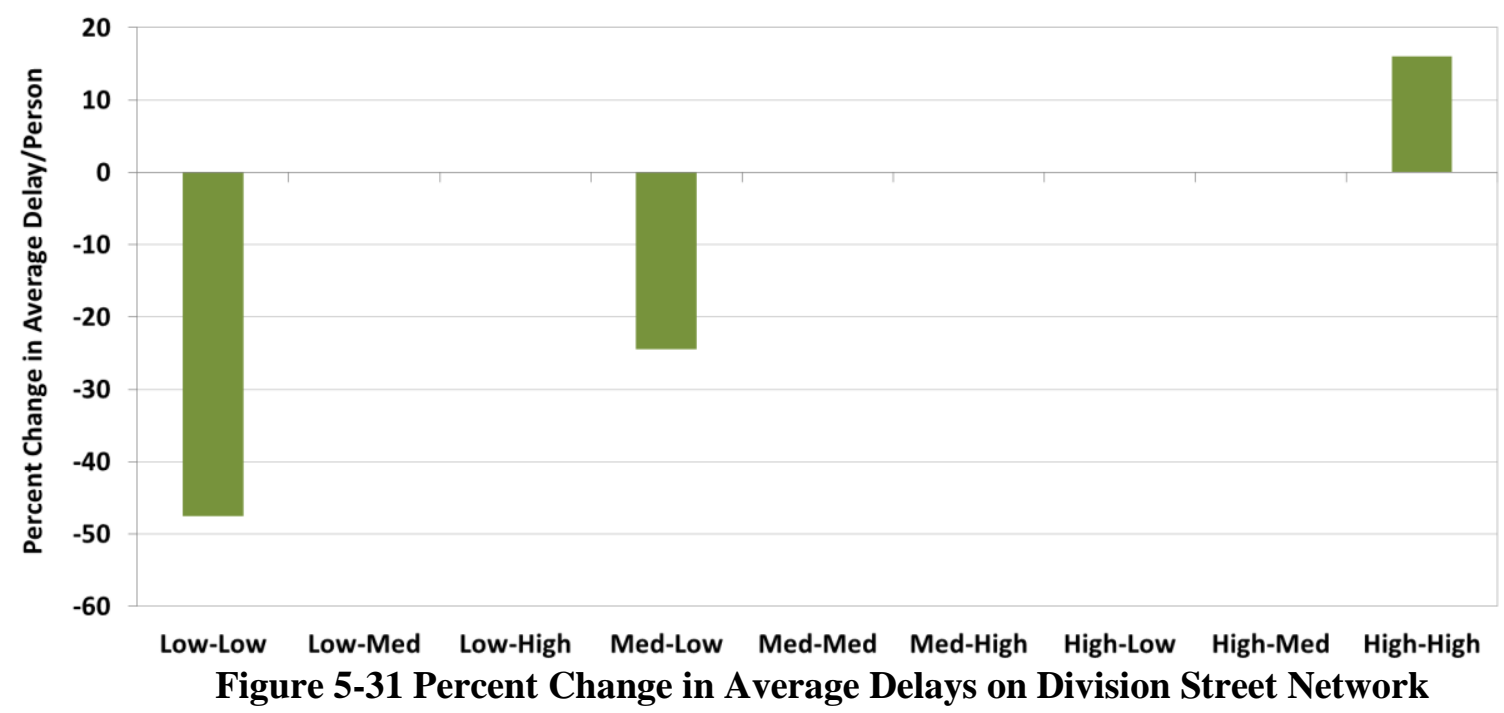

The plot above shows similar trends as seen in the hypothetical analysis on Multnomah Street. This analysis reinforces the prior findings that free operation shows benefits for light traffic conditions and coordination is preferred for heavy traffic conditions.

\subsection{Summary}

This chapter presented the findings of analysis that evaluated operational signal timing strategies related to mode of operation that could be employed at intersections to benefit pedestrians. Various combinations of volumes were used to assess the impacts of 
coordinated or free operation on all modes. Instead of average vehicle delay, overall person delay was used to study the tradeoffs of the two modes of operation.

The general finding was that free operation was always beneficial for pedestrians within the limits of the assumptions made in this research. Considering system impacts, free operation showed more benefits in terms of reduced overall delay at low volumes and the benefits of coordination are realized at higher main line volumes. During coordination, shorter cycle lengths in general are beneficial to pedestrians, confirming a prior research finding (Noland, 1996, Ishaque et al. 2006). The results also show greater reductions in delay at low volumes when fully actuated free operation is implemented.

Guidance in the form of operational strategies that allow the system operator to determine the best mode of operation was also developed during this research. The general framework can be easily applied, by knowing traffic volumes on the main line and pedestrian signal actuation frequency for the side street. Agencies often gather main line volumes to measure system performance. Methods to gather actuations and delay were outlined in section 4.3; while these methods are specific to the signal controller software used by the City of Portland, similar methods could be developed elsewhere with other software. The strategies presented in this research represent low cost approaches, which can be easily implemented with existing resources to increase efficiency. 


\section{CONCLUSIONS AND RECOMMENDATIONS}

This dissertation has made contributions in the areas of travel behavior research and traffic operations by exploring pedestrian attitudes and perceptions while crossing and designing control strategies to accommodate pedestrians at signalized intersections. The findings related to pedestrian perceptions and attitudes regarding delay, crossing time and the role of safety and compliance in crossing add to the body of literature in travel behavior research and are helpful in designing safe crossings. The control strategies are a valuable tool for system operators and signal timing engineers for accommodating multimodal users efficiently at intersections. A review of key findings along with a discussion of implications and recommendations is presented below.

\subsection{Contributions to the Literature}

The limitations of current signal timing strategies with respect to lack of pedestrian accommodation at signalized intersections were illustrated in Chapter 1. Existing signal timing design does not explicitly consider pedestrian needs or prioritize pedestrians. As discussed in Chapter 3, there is also a gap in understanding how pedestrian detection at intersections influences perceptions, attitudes and crossing behavior at intersections.

The main objectives of this research were to quantify the factors that influence pedestrian crossing behavior at signalized intersections and to develop strategies that could improve crossing behavior by reducing pedestrian delay. An intercept survey of crossing pedestrians was undertaken to understand how demographics such as gender and age, trip characteristics such as trip frequency, length and purpose and type of pedestrian 
detection at intersections impacted perceptions of satisfaction with delay and determinants of crossing decisions such as safety and/or compliance. Novel methods using existing resources to measure pedestrian delay in the signal controller were developed and deployed at various intersections in Portland, OR to understand the magnitude of delay. Both pedestrian actuations and delay were measured within the signal controller. Next, the impacts of two strategies to reduce pedestrian delay: increase in permissive length and change in mode of operation from coordinated to free were quantified. A matrix identifying optimal feasibility regimes for the two strategies was developed to help system operators with signal timing decisions at intersections that promote efficiency for all modes. To address the limitations of lack of control strategies that incorporate pedestrians and gaps in pedestrian crossing behavior research, this study offers three main contributions to the literature:

1. Provides a validated method for logging pedestrian delay per actuation using Type 2070 signal controllers and Voyage software.

2. Quantifies the impacts of demographics, trip characteristics and type of pedestrian detection (actuated or recall) at intersections on pedestrian attitudes and perceptions.

3. Provides guidance in the form of a matrix that is based on volume-capacity ratios and pedestrian actuation frequency rate for determining appropriate signal controller mode of operation for reducing pedestrian delay at signalized intersections.

These findings are discussed in the subsections that follow. 


\subsubsection{Signal Controller Data Logging}

Performance metrics are critical in determining how well the transportation system is meeting its desired goals and objectives. From a traffic operations perspective, performance measures or measures of effectiveness (MOE's) offer the engineers and system operators, the ability to quantify the performance of an intersection, segment, corridor or network. Fundamental to generating performance measures is the availability of high quality data. Recent research at Purdue University, in collaboration with Indiana Department of Transportation that has advanced and improved the signal controller's data logging capabilities has shown tremendous promise (Day et al., 2010). However, adoption of the suggestions of this research into the state of practice is lagging.

During this research, through collaborative efforts with the City of Portland's signal timing staff, methods to record pedestrian actuations and delay per actuation were developed. This thesis represents the first effort of its kind in Portland, OR, using 2070 signal controllers and W Signal's Voyage software to collect pedestrian delay per actuation. The methods developed during the course of this research, provide the opportunity to develop and analyze a number of pedestrian performance metrics such as the number of cycles with pedestrian phases, trends in pedestrian actuations (which could be a proxy for pedestrian demand) and delay measures (maximum, minimum and average delay per actuation). These measures allow the signal timing engineer to assess and evaluate the intersection from a pedestrian perspective. Agencies and cities can adapt the methodology to flag intersections that exceed certain thresholds for pedestrian delay, take remedial action such as updating signal timing and analyze the intersection performance 
post changes. In the absence of actual demand data, the actuations can be considered as a proxy for demand and can also be used for safety analysis.

Current efforts are underway at the City of Portland and signal controller software developer (NW Signal) to incorporate automated delay measurement in the signal controller for all modes of traffic. Currently the data logging methods have been implemented at 20 locations within the City of Portland. The actuation and delay data is currently being archived in PORTAL (see http://demo.portal.its.pdx.edu/Portal/index.php/pedbike). Visualizations are being developed to aid practitioners and researchers in understanding and evaluating trends in pedestrian activity at intersections.

\subsubsection{Pedestrian Crossing Behavior}

In urban areas, street crossings and resulting delay are often viewed as a deterrent for walking. Understanding pedestrian crossing behavior is fundamental to designing and operating safe facilities (Zaki et al., 2012). As summarized in Chapter 3, an intercept survey of crossing pedestrians at signalized intersections was undertaken to quantify the role of factors such as age, gender, group status, trip purpose, frequency and length and detection infrastructure on a person's decision to cross the street and their insights on satisfaction with delay. Based on modeling effort, some key findings are listed below.

- Majority of the walking trips $(70 \%)$ were found to be short $(<10$ minutes in length). Assuming a walking speed of $3.5 \mathrm{ft} / \mathrm{s}$, the distance traveled in 10 minutes 
is 0.4 miles. This confirms a finding in literature, with research showing that people generally walk no more than 0.5 miles (Noland, 1996).

- Significant differences in perceptions of safety and satisfaction with crossing time and delay with age were found. A higher proportion of younger adults perceived the intersections as safe and expressed greater dissatisfaction with delay compared to older adults. This corroborates a prior finding that older pedestrians can endure longer waiting time than younger pedestrians (Wang et al., 2011).

- Safety played a larger role in pedestrians' decision to cross the street than compliance, with a larger percentage of people agreeing with the statement that their crossing decisions were based on safety than compliance ( $85.7 \%$ vs. $59.7 \%)$.

- Significant differences in attitudes regarding crossing behavior with respect to gender were found. More women than men based their crossing decisions on considerations of both safety and compliance, the chi-square test found significant gender based differences with regards to safety and compliance considerations while crossing $(\mathrm{p}<0.05)$. This confirms a past finding in literature that noncompliance among males was higher compared to females (Diaz, 2002, Rosenbloom et al., 2009, Wang et al., 2011).

- Trip purpose was found to be a significant factor in crossing decisions. Respondents on work trips were associated with 0.37 times the odds of agreement with the statement that safety was a factor while crossing compared to respondents on shopping trips, possibly due to time constraints. Wang et al., found that trip purposes such as work or school were associated with an increased 
risk of violation (Wang et al., 2011). Similarly, respondents on recreational trips and/or executing errands were less concerned about safety while crossing, possibly due to the time constraints associated with their trips. Conversely respondents returning home were more likely to be concerned about safety because they are less time constrained.

- Group status also influences crossing decisions; people crossing in groups had $60 \%$ lower odds to consider safety while crossing. Bradbury et al. found that pedestrians were 3.7 times more likely to wait for the signal, if there were other pedestrians also present at the intersection (Bradbury et al., 2012). A similar finding was also encountered by Rosenbloom, that presence of other waiting pedestrians decreased the likelihood of non-compliance (Rosenbloom, 2009).

- Respondents who had positive perceptions of safety were less likely to consider safety as an influencing factor in crossing decisions. Many factors can influence a pedestrian's perceptions of safety. These include vehicle volumes and speeds, crossing time and distance, lighting conditions along the roadway and familiarity with intersections.

- Respondents who stated that their trip lengths were longer (> $15 \mathrm{~min}$ ) were less likely to consider safety while crossing compared to respondents on short trips $(<$ $5 \mathrm{~min}$ ). Respondents on longer trips were probably using walking as their exclusive mode of transportation. With respondents on shorter trips, walking was possibly used in conjunction with other transportation modes, as a link between the transportation system network and the origin or destination of their trip. 
People on longer trips were probably less concerned with safety because their waiting times would accrue at each intersection along their route leading to longer trip times, which is not desirable.

- Many factors influenced the likelihood of a pedestrian's satisfaction with delay. Younger adults, public transportation users and pedestrians on short trips were less likely to be satisfied with delay.

- Type of pedestrian infrastructure at intersections (recall vs. actuated phases) also influenced a pedestrian's likelihood of satisfaction with delay. Pedestrians crossing at intersections with recall phases were associated with 5-7 times the odds of being satisfied with delay compared to actuated intersections.

\subsubsection{Pedestrian Responsive Signal Timing Strategies}

Micro-simulation models are effective tools to study the impact tradeoffs between modes for multimodal analysis. The micro-simulation software VISSIM was used to create a network consisting of signalized intersections and auto, bicycle and pedestrian volumes were varied hypothetically to determine the optimal regimes for signal controller mode of operation (coordinated, free) based on minimization of per person delay. A case study to test the transferability of findings was also conducted on a different corridor. Significant findings are presented below.

- Free operation is generally beneficial for pedestrians as the signals are more responsive and less constrained when compared to coordinated operations. Average delay reductions for $7-18 \%$ were observed for pedestrians during free 
operation as compared to the coordinated operation on Multnomah Street network.

- Coordination primarily benefits major street through movements (auto and bicycle). Side street movements (auto and ped) were found to have higher delays compared to main street movements.

- Treating all users equally, free operation is associated with overall larger delay savings when traffic volumes are low or moderate $(\mathrm{V} / \mathrm{C}$ ratios for the main line through movements are less than 0.7). The greatest benefit of free operation is realized during the low volume regime (auto main line $\mathrm{V} / \mathrm{C}<0.3$ and rate of ped phase frequency $<0.3$ ) with a $32 \%$ reduction in overall delay as compared to the coordinated operation. Free signal operation during low mainline traffic volumes also benefits side street vehicle movements via reductions in delay, in addition to pedestrian delay reductions.

- Treating all users equally, coordinated operation is associated with overall larger delay savings when main line $\mathrm{V} / \mathrm{C}$ ratios are greater than 0.7 . The greatest benefit of coordinated operation is realized during the high volume regime (auto main line $\mathrm{V} / \mathrm{C}>0.7$ and rate of ped phase frequency $=100 \%$ ) with a $28 \%$ reduction in overall delay as compared to the coordinated operation.

- Generally, higher cycle lengths are associated with larger delays for the side street movements (auto and pedestrian) and lower delays for the coordinated movements. 
- The greatest benefit of detection on all approaches is observed during the low volume regime with reductions in average delay ranging between 0 and $22 \%$ when delays are compared between free and fully actuated free operations.

\subsection{Implications and Recommendations}

There are many policy implications and specific recommendations stemming from this research, which are discussed in the following subsections.

\subsubsection{Using Existing Resources to Leverage Performance Measurement}

There is growing interest in performance measurement for arterial operations. These measures often require high quality, high resolution data. Visualization tools allow the users to graphically assess system performance and identify problem locations. Currently many agencies collect little/no data from the controller, which in turn inhibits performance measurement. Signal system data, if at all collected, are stored for a brief time and eventually discarded; very few agencies archive arterial data. Archiving arterial data allows users to see trends in performance measures across modes and analyze the effects of multiple strategies at an intersection or corridor level. Arterial data could also be used to compare travel times across modes. Cities and agencies can benefit from leveraging existing resources to collect and archive arterial data as discussed in Chapter 4. 


\subsubsection{Signal Timing for all Users}

The traditional practice of prioritizing vehicles over users of all other modes should be reevaluated in the context of livability. As communities engage in efforts to improve livability and aim to transform their streets into vibrant public spaces that foster interactions, a new approach that balances user needs is necessary to accommodate all modes and improve safety for all users.

The quality of the transportation system in part is influenced by signal timing. Operation decisions at intersections play a role in travel behavior and mode choice. Agencies and cities should evaluate their policies regarding coordination of traffic signals and maximum delay thresholds for each mode. As this research suggests, coordination is beneficial during peak periods, when mainline auto volumes are high. During coordination, pedestrian friendly strategies such as keeping cycle lengths short to minimize delay for side street pedestrians and other modes and allowing the signal to leave coordination to serve the pedestrian when the situation is warranted should be considered. Coordinating signals could also result in higher vehicular speeds, which are unsafe for pedestrians. Therefore, timing signals to allow progression at low vehicular speeds can discourage speeding and lead to a safer environment (NACTO, 2014).

Allowing certain signals to run free during certain time periods in a day, such as the noon hour, when there is high pedestrian activity, can reduce delays for pedestrians and also help promote walking.

Current pedestrian accommodation at signalized intersections has been primarily viewed through a safety lens, without any consideration for delay. While trade-offs exist 
between safety and efficiency at intersections, both must be considered when accommodating pedestrians (Vallyon et al., 2011). Although the control strategies evaluated in this research are primarily efficiency based, there are a few safety implications to consider. Studies have shown a link between signal non-compliance and pedestrian crashes (Zeeger, 1985, Shinar, 2008) and between non-compliance and delay (HCM 2010). Therefore reducing pedestrian delay can result in both efficiency and safety benefits. In 2012, 70\% of pedestrian fatalities occurred at night and older pedestrians accounted for $20 \%$ of all pedestrian fatalities (NHTSA, 2012). Typical conditions at night include low traffic volumes with gaps resulting in higher motor vehicle speeds and poor light conditions making it harder for drivers to see pedestrians. The inherent risk to a pedestrian who violates signal indications at night is higher than during day. Allowing the signals to be more responsive at night, by operating in uncoordinated mode during low traffic volume conditions, can result in lowered delays for pedestrians, which in turn promotes signal compliance and leads to improved safety.

The findings from this research as based on the consideration of equal value of time for all users. Since pedestrians are the most vulnerable users of the transportation system, an alternative approach could conceivably provide the highest priority to pedestrians at intersections. This greater accommodation of pedestrians could lead to higher pedestrian volumes due to latent demand. This could also spur research into additional control strategies that seek to balance both safety and efficiency for pedestrians. 


\subsubsection{The Three E's for Pedestrian Safety}

The role of engineering, education and enforcement in traffic safety improvements are well known. Effective measures often combine all three tools to enhance safety.

Engineering measures such as shorter crossings designed to reduce pedestrian exposure, using pedestrian recall feature at intersections with high pedestrian volumes, providing accessible pushbuttons with auditory/visual feedback for actuated pedestrian phases, and improving signage, can promote signal compliance and reduce risky behaviors. While operational decisions at the intersection level influence pedestrian crossing behavior, other demographic and social factors also play a role. Analysis of survey data in this study revealed that men and people crossing in groups are less concerned about compliance while crossing. In addition, young adults are also less satisfied with waiting time to cross. Young children and older adults, who were not captured in this study, are vulnerable populations, who are traditionally more at risk and more dependent on walking. Targeted education measures and public education campaigns designed to increase awareness of safe crossing behavior among these segments of the population can help in promoting safety. Educating drivers on yielding laws and compliant behavior may also improve the overall safety at intersections. High visibility enforcement can serve as a deterrent for non-compliant behavior by motorists; several studies have reported an increase in drivers yielding behavior and a reduction in crashes (Britt et al. 1995, Van Houten et al. 2013). In addition, enforcing rules for pedestrians can also be beneficial in reducing adverse outcomes. 


\subsection{Areas for Future Research}

There are several areas for future research that have arisen as a result of this research. As interest in the concept of "complete streets" grows, the idea of accommodating pedestrians and other users safely, without disproportionately increasing their delay is gaining traction. More research is needed in the following areas:

- Continuing investigation on pedestrian control strategies at intersections

- Safety vs. efficiency tradeoffs for various pedestrian strategies

- Effect of increased permissive length on other modes

- Evaluation of priority pedestrian service and investigation of feasibility regimes, where it may be warranted 


\section{REFERENCES}

Abrams, C. M., and S. A. Smith. Selection of Pedestrian Signal Phasing. In Transportation Research Record: Journal of the Transportation Research Board, No. 629, Transportation Research Board of the National Academies, Washington, D.C., 1977, pp. 1-6.

Akcelik, R. Microsimulation and Analytical Models for Traffic Engineering. Presentation at the ARRB-AUSTROADS Micro simulation Forum, September 2007.

Ayuso, M. Guillen, M., and M. Alcaz. The Impact of Traffic Violations on the Estimated Cost of Traffic Accidents with Victims. Accident Analysis and

Prevention, Volume 42, No. 2, 2010, pp.709-717.

Bechtel, A., K. MacLeod, and D. Ragland. Pedestrian Scramble Signal in Chinatown Neighborhood of Oakland, California: An Evaluation. In Transportation Research Record: Journal of the Transportation Research Board, No. 1878, Transportation Research Board of the National Academies, Washington, D.C., 2004, pp. 19-26.

Berkow, M., Monsere, C.M., Koonce, P., Bertini, R.L. and Wolfe, M. Prototype for Data Fusion Using Stationary and Mobile Data Sources for Improved Arterial Performance Measurement. In Transportation Research Record: Journal of the Transportation Research Board, No. 2099, Transportation Research Board of the National Academies, Washington, D.C., 2009, pp. 102-112.

Bhattacharya, P. and Virkler, M. Optimization for Pedestrian and Vehicular Delay in a Signal Network. In Transportation Research Record: Journal of the Transportation Research Board, No. 1939, Transportation Research Board of the National Academies, Washington, D.C., 2005, pp. 115-122.

Braun, R. and Roddin, M. Quantifying the Benefits of Separating Pedestrians and Vehicles. NCHRP Report 189, Transportation Research Board, Transportation Research Board of the National Academies, Washington DC., 1978.

Bradbury, K., Stevens, J., Boyle, L., and Rutherford, S. To Go or Not To Go Pedestrian Behavior at Intersections with Standard Pedestrian Call Buttons. In Transportation Research Record: Journal of the Transportation Research Board, No. 2299, Transportation Research Board of the National Academies, Washington, D.C., 2012, pp. 174-179. 
Britt, J.W., Bergman, A.B., and Moffat, J. Law Enforcement, Pedestrian Safety amd Driver Compliance with Crosswalk Laws: Evaluation of a Four Year Campaign in Seattle, Washington. In Transportation Research Record: Journal of the Transportation Research Board, No. 1485, Transportation Research Board of the National Academies, Washington, D.C., 1995, pp. 160-167.

Byrne, N., P. Koonce, R.L. Bertini, C. Pangilinan and M. Lasky. Using Hardware-in-the-Loop Simulation to Evaluate Signal Control Strategies for Transit Signal Priority. In Transportation Research Record No. 1925, Transportation Research Board of the National Academies, Washington, D.C., 2005, pp. 227-234.

Cohen, J., Cohen, P., West, S.G. and L.S. Aiken. Applied Multiple Regression/Correlation Analysis for the Behavioral Sciences, Third Edition.

Day, C.M. and E. Smaglik. Quantitative Evaluation of Fully Actuated Versus Non-Actuated Coordinated Phases. Proceedings of the 87th Annual Meeting of the Transportation Research Board, Washington D.C., January 2007.

Day, C.M., Premachandra, H., and Bullock, D.M. Rate of Pedestrian Signal Phase Actuation as a Proxy Measurement of Pedestrian Demand. Proceedings of the $91^{\text {st }}$ Annual Meeting of the Transportation Research Board, Washington D.C., January 2011.

E.M. Diaz. Theory of Planned Behavior and Pedestrians' Intention to Violate Traffic Regulations. Transportation Research Part F, Volume 5, 2002, pp. 169175.

Dunn, R. and R. Petty. Mid-Block Pedestrian Crossings - An Examination of Delay. Proceedings of the $12^{\text {th }}$ Annual Australian Road Research Board Conference, Hobart, Tasmania, Aug, 1984.

Eccles, K. A., R. Tao, and B. C. Mangum. Evaluation of Pedestrian Countdown Signals in Montgomery County, Maryland. In Transportation Research Record: Journal of the Transportation Research Board,No. 1878, Transportation Research Board of the National Academies, Washington, D.C., 2004, pp. 36-41.

Fayish, A. C. and F. Goss. Safety Effectiveness of Leading Pedestrian Intervals Evaluated by a Before-After Study with Comparison Groups. In Transportation Research Record: Journal of the Transportation Research Board, No. 2198,TRB, National Research Council, Washington, D.C., 2010, pp. 15-22. 
Federal Highway Administration. Manual of Uniform Traffic Control Devices, 2009.

Federal Highway Administration. The National Bicycling and Walking Study. 15Year Status Report. Accessed on July 27 $7^{\text {th }}, 2013$.

Fruin, J.J. Pedestrians: Planning and Design, Revised Edition 1987.

Gårder, P. Pedestrian Safety at Traffic Signals: A Study Carried Out with the Help of a Traffic Conflicts Technique. Accident Analysis and Prevention, Vol. 21, No. 5, 1989, pp. 435-444.

Hamed, M. Analysis of Pedestrians' Behavior at Pedestrian Crossings. Safety Science, Vol. 38, No. 1, 2001, pp. 63-82.

Highway Capacity Manual 2010, Transportation Research Board of the National Academies, Washington D.C.

Hubbard, S., Bullock, D.M. and C.M. Day. Integration of Real-Time Pedestrian Performance Measures into Existing Infrastructure of Traffic Signal System. In Transportation Research Record: Journal of the Transportation Research Board, No. 2080, Transportation Research Board of the National Academies, Washington, D.C., 2008, pp. 37-47.

Hughes, R., H. Huang, C. Zegeer, and M. Cynecki. Automated Detection of Pedestrians in Conjunction with Standard Pedestrian Push Buttons at Signalized Intersections. In Transportation Research Record: Journal of the Transportation Research Board, No. 1705, Transportation Research Board of the National Academies, Washington, D.C., 2000, pp. 32-39.

Hyde-Wright, A., Graham, B. and K. Nordback. Counting Bicyclists with Pneumatic Tube Counters on Shared Roadways. In ITE Journal, Institute of Transportation Engineers, 2014, pp. 32-37.

Ishaque, M. M. Policies for Pedestrian Access: A Multi-Modal Tradeoff Analysis Using Micro-Simulation Techniques. PhD dissertation. University of London, 2006.

Ishaque, M. M., and R. B. Noland. Multimodal Microsimulation of Vehicle and Pedestrian Signal Timings. In Transportation Research Record: Journal of the Transportation Research Board, No. 1939, Transportation Research Board of the National Academies, Washington, D.C., 2005, pp. 107-114. 
Karkee, G., Pulugurtha, S., and S. Nambisan. Statistical Analysis of Pedestrian Crossing Behavior on Streets. Proceedings of the $88^{\text {th }}$ Annual Meeting of the Transportation Research Board, Washington, D.C., 2009.

Kattan, L., Acharjee, S. and R. Tay. Pedestrian Scramble Operations. Pilot Study in Calgary, Alberta, Canada. In Transportation Research Record 2140, Transportation Research Board of the National Academies, Washington, D.C., 2009, pp. 79-84.

Keegan, Owen, and Margaret O’Mahony. Modifying Pedestrian Behavior. In Transportation Research Part A: Policy and Practice 37, no. 10, 2003, pp. 889901.

Kitamura, R., Mokhtarian, P.L., and L. Laidet. A Micro-Analysis of Land Use and Travel in Five Neighborhoods in the San Francisco Bay Area. In Transportation, Volume 24, 1997, pp. 125-158.

Knoblauch, R.L., Pietrucha, M.T., and M. Nitzburg. Field Studies of Pedestrian Walking Speed and Start-Up Time. In Transportation Research Record: Journal of the Transportation Research Board, No. 1538, 1996, pp. 27-38.

Koonce, P., Rodegerdts, L., Lee, K., Quayle, S., Beaird. S., Braud, C., Bonneson, K., Tarnoff, P. and T. Urbanik. Traffic Signal Timing Manual. Accessed at http://ops.fhwa.dot.gov/publications/fhwahop08024/index.htm

Kothuri, S., Reynolds, T., Monsere, C. and P. Koonce. Preliminary Development of Methods to Automatically Gather Bicycle Counts and Pedestrian Delay at Signalized Intersections. In Proceedings of the $90^{\text {th }}$ Annual Meeting of the Transportation Research Board, Transportation Research Board of the National Academies, Washington D.C., 2012.

Kothuri, S., Reynolds, T., Monsere, C. and P. Koonce. Testing Strategies to Reduce Pedestrian Delay at Signalized Intersections: A Pilot Study in Portland, Oregon. In Proceedings of the $92^{\text {nd }}$ Annual Meeting of the Transportation Research Board, January 13-17, 2013.

Koonce, P., Rodegerdts, L., Lee, K., Quayle, S., Beaird, S., Braud, C., Bonneson, J., Tarnoff, P. and Urbanik, T. Traffic Signal Timing Manual, Federal Highway Administration, 2008.

Little, J. D. C., M. D. Kelson, and N. H. Gartner. MAXBAND: A Program for Setting Signals on Arterials and Triangular Networks. In Transportation Research 
Record: Journal of the Transportation Research Board, No.795, Transportation Research Board of the National Academies, Washington, D.C., 1981, pp. 40-46.

Liu, H. and W. Ma. A Virtual Vehicle Probe Model for Time-Dependent Travel Time Estimation on Signalized Arterials, In Transportation Research Part C: Emerging Technologies, No. 17, Issue 1, TRB, National Research Council, Washington, D.C., 2009, pp. 11-26.

Macgregor, C., Smiley, A., and W. Dunk. Identifying Gaps in Child Pedestrian Safety: Comparing What Children Do with What Parents Teach. In

Transportation Research Record: Journal of the Transportation Research Board, No. 1674, Transportation Research Board of the National Academies, Washington DC, 1999, pp. 32-40.

National Association of City Transportation Officials. Urban Street Design Guide, 2013.

National Highway Traffic Safety Administration. Traffic Safety Facts, 2011 Data. Accessed from http://www-nrd.nhtsa.dot.gov/Pubs/811748.pdf

National Highway Traffic Safety Administration. Traffic Safety Facts, 2012 Data. Accessed from http://www-nrd.nhtsa.dot.gov/Pubs/811888.pdf

National Highway Traffic Safety Administration (NHTSA). National Survey of Bicyclist and Pedestrian Attitudes and Behavior. Volume II Findings Report, 2008. Accessed from http://www.nhtsa.gov/Driving+Safety/Research+\&+Evaluation/National+Survey + of + Bicyclist+and + Pedestrian + Attitudes + and + Behavior

Noland, R. B. Optimal Travel Time Trade-Offs at Signalized Pedestrian Crossings. Center for Transport Studies, Department of Civil and Environmental Engineering, Imperial College, London, 2003.

Noland, R. Pedestrian Travel Times and Motor Vehicle Traffic Signals. In Transportation Research Record: Journal of the Transportation Research Board, No. 1553, Transportation Research Board of the National Academies, Washington DC, 1996, pp. 28-33.

Oregon Department of Transportation. Protocol for VISSIM Simulation, June 2011. 
Pedestrian Safety Countermeasure Deployment Project. FHWA, U.S. Department of Transportation.

http://safety.fhwa.dot.gov/ped_bike/ped/ped_scdproj/sf_ch1.htm.

Pretty, R. The Delay to Pedestrians and Vehicles at Signalized Intersections. ITE Journal, May 1979, pp. 20-23.

Quayle, S.M., Koonce, P., DePencier, D., and D.M. Bullock. Arterial Performance Measures with Media Access Control Readers Portland, Oregon Pilot Study. In Transportation Research Record: Journal of the Transportation Research Board, No. 2192, Transportation Research Board of the National Academies, Washington, D.C., 2010, pp. 185-193.

Roshandeh, A.M., Levinson, H.S., Li, Z., Patel, H., and Zhou, B. New Methodology for Intersection Signal Timing Optimization to Simultaneously Minimize Vehicle and Pedestrian Delays. In Journal of Transportation Engineering, American Society of Civil Engineers, 2013, pp. 0401400904014009-10.

Rosenbloom, T. Crossing at a Red Light: Behavior of Individuals and Groups. In Transportation Research Part F, Vol 12, 2009, pp. 389-394.

Shinar, David. Traffic Safety and Human Behavior. Emerald Group Publishing, Ltd., 2008.

Skabardonis, A. and N. Geroliminis, Real-time Monitoring and Control on Signalized Arterials, Journal of Intelligent Transportation Systems, June 2008, Vol. 12, No. 2, pp. 64-74.

Struckman-Johnson, D., Lund, A., Williams, A. and D. Osborne. Comparative Effects of Driver Improvement Programs on Crashes and Violations. Accident Analysis and Prevention, Volume 21, No.3, 1989, pp. 203-215.

Tarnoff, P.J., J. S. Wasson, S.E. Young, M. Ganig, D.M. Bullock, and J.R. Sturdevant. The Continuing Evolution of Travel Time Data Information Collection and Processing. Proceedings of the $88^{\text {th }}$ Annual Meeting of the Transportation Research Board, TRB, National Research Council, Washington, DC, 2009.

Tian, Z. Z., T. Urbanik, R. Engelbrecht, and K. Balke. Pedestrian Timing Alternatives and Impacts on Coordinated Signal Systems Under Split-Phasing Operations. In Transportation Research Record: Journal of the Transportation 
Research Board, No. 1748, Transportation Research Board of the National Academies, Washington, D.C., 2001, pp. 46-54.

Santos, A., McGuckin, N., Nakamoto, H Y., Gray, D. and Liss, S. Summary of Travel Trends : 2009 Household Travel Survey. Accessed from http://nhts.ornl.gov/2009/pub/stt.pdf

S.M. Turner. Advanced Techniques for Travel Time Data Collection. In Transportation Research Record: Journal of the Transportation Research Board, No. 1551, Transportation Research Board of the National Academies, Washington, D.C., 1996, pp. 51-58.

P. Turner. Making Crosswalks Safer for Pedestrians. Project Report, Center for Urban Transportation Research, University of South Florida, Tampa, 2000.

Vallyon, C. and S. Turner. Reducing Pedestrian Delay at Traffic Signals. New Zealand Transport Agency Research Report 440, 2011. Accessed from http://www.trb.org/Main/Blurbs/165672.aspx

Van Houten, R., R. A. Retting, C. M. Farmer, and J. Van Houten. Field Evaluation of a Leading Pedestrian Interval Signal Phase at Three Urban Intersections. In Transportation Research Record: Journal of the Transportation Research Board, No. 1734, Transportation Research Board of the National Academies, Washington, D.C., 2000, pp. 86-92.

Van Houten, R., R. D. Ellis, Jr., J. Sanda, and J.-L. Kim. PedestrianPush-Button Confirmation Increases Call Button Usage and Compliance. In Transportation Research Record: Journal of the Transportation Research Board, No. 1982, Transportation Research Board of the National Academies, Washington, D.C., 2006, pp. 99-103.

Van Houten, R., Malenfant, L., Huitema, B. and R. Blomberg. The Effects of High Visibility Enforcement on Driver Compliance to Pedestrian Yield Right-ofWay Laws. In Transportation Research Record: Journal of the Transportation Research Board, No. 2393, Transportation Research Board of the National Academies, Washington, D.C., 2013, pp. 41-49.

M.R. Virkler. Pedestrian Compliance Effects on Signal Delay. In Transportation Research Record: Journal of the Transportation Research Board, No. 1636, Transportation Research Board of the National Academies, Washington D.C., 1998, pp. 88-91. 
Wang, X. and Z. Tian. Pedestrian Delay at Signalized Intersections with a TwoStage Crossing Design. In Transportation Research Record: Journal of the Transportation Research Board, No. 2173, Transportation Research Board of the National Academies, Washington, D.C., 2010, pp. 133-138.

Wang, W., Guo, H., Gao, Z. and H. Bubb. Individual Differences of Pedestrian Behavior in Midblock Crosswalk and Intersection. International Journal of Crashworthiness Vol 16, No. 1, 2011, pp. 1-9.

F.V. Webster. Traffic Signal Settings, Road Research Technical Paper no. 39, Transport Research Laboratory, Berkshire, 1958.

Yanfeng, W., Z. Shunying, W. Hong, L. Bing, and L. Mei. Characteristic Analysis of Violation Crossing Behavior Based on Logistics Model. Proc., International Conference on Intelligent Computation Technology and Automation, Changsha, China, Vol. 1, IEEE, Los Alamitos, Calif., 2010, pp. 926928.

Zaidel, D. M., and I. Hocherman. Safety of Pedestrian Crossings at Signalized Intersections. In Transportation Research Record 1141, Transportation Research Board of the National Academies, Washington, D.C., 1987, pp. 1-6.

Zaki, M.H., Sayed, T., Ismail, K. and F. Alrukaibi. Identification of Pedestrians Non-Conforming Behavior at Urban Intersections using Computer Vision. In Transportation Research Record: No. 2279, Transportation Research Board of the National Academies, Washington DC, 2012, pp. 54-64.

Zeeger, C.V., Opiela, K.S., and Cynecki, M.J. Pedestrian Signalization Alternatives. Federal Highway Administration, July 1985.

Zegeer, C. V., and M. J. Cynecki. Determination of Motorist Violations and Pedestrian-Related Countermeasures Related to Right-Turn-on Red. In Transportation Research Record 1010, Transportation Research Board of the National Academies, Washington, D.C., 1985, pp. 16-28.

Zhang, H. M. Link-Journey-Speed Model for Arterial Traffic. In Transportation Research Record: Journal of the Transportation Research Board, No. 1676, TRB, National Research Council, Washington, D.C., 1999, pp. 109-115. 


\section{APPENDIX}

Table A-1 Statistical Comparison of Delays for Low Auto Volume Scenarios with Existing Signal Timing for the Multnomah Street Network

\begin{tabular}{l|rrrr}
\hline Scenario & Simulation Runs & Coordinated Delay & Free Delay & p-value \\
\hline \multirow{5}{*}{ Low Auto - Low Ped } & Run 1 & 12.6 & 8.3 & \\
& Run 2 & 13.3 & 9.0 & \\
& Run 3 & 13.3 & 8.7 & \\
& Run 4 & 13.0 & 8.9 & \\
& Run 5 & 12.5 & 8.4 & $3.49 \mathrm{E}^{-14}$ \\
& Run 6 & 13.2 & 9.2 & \\
& Run 7 & 12.6 & 9.2 & \\
& Run 8 & 13.1 & 8.6 & \\
& Run 9 & 12.1 & 8.1 & \\
& Run 10 & 12.2 & 8.1 \\
\hline \multirow{5}{*}{ Low Auto- Med Ped } & Run 1 & 16.3 & 14.2 & \\
& Run 2 & 15.9 & 14.3 & \\
& Run 3 & 16.6 & 13.9 & \\
& Run 4 & 17.3 & 14.7 & \\
& Run 5 & 17.0 & 14.4 & \\
& Run 6 & 16.1 & 14.1 & $1.72 \mathrm{E}^{-08}$ \\
& Run 7 & 17.0 & 14.2 & \\
& Run 8 & 16.5 & 14.2 & \\
& Run 9 & 17.2 & 14.0 & \\
& Run 10 & 15.8 & 13.9 & \\
& Run 1 & 20.2 & 17.7 & \\
& Run 2 & 20.1 & 17.6 & \\
& Run 3 & 19.7 & 18.1 & \\
& Run 4 & 19.3 & 17.7 & \\
& Run 5 & 20.0 & 17.9 & \\
& Run 6 & 20.5 & 18.0 & $1.13 \mathrm{E}^{-09}$ \\
& Run 7 & 20.4 & 17.5 & \\
& Run 8 & 20.2 & 17.8 & \\
& Run 9 & 20.0 & 18.1 & \\
& Run 10 & 19.3 & 17.6 &
\end{tabular}


Table A-2 Statistical Comparison of Delays for the Medium Auto Volume Scenarios with Existing Signal Timing on Multnomah Street Network

\begin{tabular}{l|rrrr}
\hline Scenario & Simulation Runs & Coordinated Delay & Free Delay & p-value \\
\hline & Run 1 & 13.7 & 10.5 & \\
& Run 2 & 13.5 & 10.8 & \\
Run 3 & 14.2 & 11.1 & \\
& Run 4 & 13.1 & 11.1 & \\
Med Auto - Low Ped & Run 5 & 13.5 & 10.7 & \\
& Run 6 & 13.7 & 10.6 & $6.23 \mathrm{E}^{-19}$ \\
& Run 7 & 13.7 & 11.5 & \\
& Run 8 & 13.9 & 11.5 & \\
& Run 9 & 13.8 & 10.9 & \\
& Run 10 & 14.3 & 11.0 & \\
& Run 1 & 16.4 & 15.5 & \\
& Run 2 & 16.2 & 15.6 & \\
& Run 3 & 16.7 & 15.6 & \\
& Run 4 & 16.8 & 14.9 & \\
& Run 5 & 16.3 & 15.0 & \\
& Run 6 & 16.6 & 15.1 & $2.88 \mathrm{E}^{-06}$ \\
& Run 7 & 16.3 & 15.6 & \\
& Run 8 & 17.2 & 14.2 & \\
& Run 9 & 16.6 & 14.4 & \\
& Run 10 & 15.8 & 14.5 & \\
& Run 1 & 20.0 & 18.3 & \\
& Run 2 & 20.3 & 18.5 & \\
& Run 3 & 20.4 & 19.0 & \\
& Run 4 & 20.6 & 18.4 & \\
& Run 5 & 20.0 & 18.3 & \\
& Run 6 & 19.8 & 18.8 & $1.32 \mathrm{E}^{-08}$ \\
& Run 7 & 19.5 & 18.4 & \\
& Run 8 & 19.8 & 18.5 & \\
& Run 9 & 19.7 & 18.5 & \\
& Run 10 & 19.8 & 19.1 & \\
& & &
\end{tabular}


Table A-3 Statistical Comparison of Delays for the High Auto Volume Scenarios with Existing Signal Timing on Multnomah Street Network

\begin{tabular}{|c|c|c|c|c|}
\hline Scenario & Simulation Runs & Coordinated & Free & p-value \\
\hline \multirow{10}{*}{ High Auto - Low Ped } & Run 1 & 16.7 & 15.6 & \multirow{10}{*}{$1.01 \mathrm{E}^{-06}$} \\
\hline & Run 2 & 15.5 & 15.1 & \\
\hline & Run 3 & 16.9 & 14.5 & \\
\hline & Run 4 & 16.0 & 14.4 & \\
\hline & Run 5 & 16.1 & 14.4 & \\
\hline & Run 6 & 16.8 & 14.0 & \\
\hline & Run 7 & 15.9 & 15.6 & \\
\hline & Run 8 & 16.6 & 14.7 & \\
\hline & Run 9 & 16.4 & 14.5 & \\
\hline & Run 10 & 16.8 & 14.6 & \\
\hline \multirow{10}{*}{ High Auto - Med Ped } & Run 1 & 19.3 & 22.6 & \multirow{10}{*}{0.02} \\
\hline & Run 2 & 19.9 & 21.8 & \\
\hline & Run 3 & 20.4 & 21.7 & \\
\hline & Run 4 & 19.4 & 18.6 & \\
\hline & Run 5 & 19.7 & 20.4 & \\
\hline & Run 6 & 19.7 & 18.9 & \\
\hline & Run 7 & 19.3 & 21.8 & \\
\hline & Run 8 & 19.5 & 19.9 & \\
\hline & Run 9 & 18.9 & 24.5 & \\
\hline & Run 10 & 19.7 & 22.3 & \\
\hline \multirow{10}{*}{ High Auto - High Ped } & Run 1 & 21.9 & 24.1 & \multirow{10}{*}{0.00} \\
\hline & Run 2 & 22.9 & 30.1 & \\
\hline & Run 3 & 21.3 & 26.1 & \\
\hline & Run 4 & 22.1 & 36.1 & \\
\hline & Run 5 & 22.0 & 25.7 & \\
\hline & Run 6 & 21.8 & 36.3 & \\
\hline & Run 7 & 21.7 & 27.0 & \\
\hline & Run 8 & 22.2 & 25.9 & \\
\hline & Run 9 & 22.5 & 24.5 & \\
\hline & Run 10 & 21.7 & 25.4 & \\
\hline
\end{tabular}


Table A-4 Statistical Comparison of Delays for Low Auto Volume Scenarios with Optimized Signal Timing (80s CL) for the Multnomah Street Network

\begin{tabular}{|c|c|c|c|c|}
\hline Scenario & Simulation Runs & Coordinated & Free & p-value \\
\hline \multirow{10}{*}{ Low Auto - Low Ped } & Run 1 & 13.3 & 8.3 & \multirow{10}{*}{$2.53 \mathrm{E}^{-13}$} \\
\hline & Run 2 & 13.7 & 9.0 & \\
\hline & Run 3 & 13.9 & 8.7 & \\
\hline & Run 4 & 13.1 & 8.9 & \\
\hline & Run 5 & 12.5 & 8.4 & \\
\hline & Run 6 & 13.4 & 9.2 & \\
\hline & Run 7 & 12.8 & 9.2 & \\
\hline & Run 8 & 13.3 & 8.6 & \\
\hline & Run 9 & 12.6 & 8.1 & \\
\hline & Run 10 & 12.2 & 8.1 & \\
\hline \multirow{10}{*}{ Low Auto - Med Ped } & Run 1 & 16.9 & 14.2 & \multirow{10}{*}{$4.08 \mathrm{E}^{-13}$} \\
\hline & Run 2 & 16.3 & 14.3 & \\
\hline & Run 3 & 16.5 & 13.9 & \\
\hline & Run 4 & 17.4 & 14.7 & \\
\hline & Run 5 & 17.1 & 14.4 & \\
\hline & Run 6 & 16.7 & 14.1 & \\
\hline & Run 7 & 16.9 & 14.2 & \\
\hline & Run 8 & 17.2 & 14.2 & \\
\hline & Run 9 & 16.7 & 14.0 & \\
\hline & Run 10 & 17.1 & 13.9 & \\
\hline \multirow{10}{*}{ Low Auto - High Ped } & Run 1 & 20.6 & 17.7 & \multirow{10}{*}{$2.44 \mathrm{E}^{-13}$} \\
\hline & Run 2 & 20.3 & 17.6 & \\
\hline & Run 3 & 20.1 & 18.1 & \\
\hline & Run 4 & 20.1 & 17.7 & \\
\hline & Run 5 & 20.4 & 17.9 & \\
\hline & Run 6 & 20.7 & 18.0 & \\
\hline & Run 7 & 20.6 & 17.5 & \\
\hline & Run 8 & 20.9 & 17.8 & \\
\hline & Run 9 & 20.9 & 18.1 & \\
\hline & Run 10 & 20.1 & 17.6 & \\
\hline
\end{tabular}


Table A-5 Statistical Comparison of Delays for Medium Auto Volume Scenarios with Optimized Signal Timing (80s CL) for the Multnomah Street Network

\begin{tabular}{|c|c|c|c|c|}
\hline Scenario & Simulation Runs & Coordinated & Free & p-value \\
\hline \multirow{10}{*}{ Med Auto - Low Ped } & Run 1 & 13.6 & 10.5 & \multirow{10}{*}{$1.56 \mathrm{E}^{-13}$} \\
\hline & Run 2 & 14.1 & 10.8 & \\
\hline & Run 3 & 13.9 & 11.1 & \\
\hline & Run 4 & 13.8 & 11.1 & \\
\hline & Run 5 & 13.6 & 10.7 & \\
\hline & Run 6 & 13.9 & 10.6 & \\
\hline & Run 7 & 13.8 & 11.5 & \\
\hline & Run 8 & 13.9 & 11.5 & \\
\hline & Run 9 & 14.7 & 10.9 & \\
\hline & Run 10 & 14.4 & 11.0 & \\
\hline \multirow{10}{*}{ Med Auto - Med Ped } & Run 1 & 17.0 & 15.5 & \multirow{10}{*}{$9.63 \mathrm{E}^{-07}$} \\
\hline & Run 2 & 16.4 & 15.6 & \\
\hline & Run 3 & 16.9 & 15.6 & \\
\hline & Run 4 & 16.4 & 14.9 & \\
\hline & Run 5 & 16.8 & 15.0 & \\
\hline & Run 6 & 17.0 & 15.1 & \\
\hline & Run 7 & 16.3 & 15.6 & \\
\hline & Run 8 & 17.0 & 14.2 & \\
\hline & Run 9 & 16.5 & 14.4 & \\
\hline & Run 10 & 15.9 & 14.5 & \\
\hline \multirow{10}{*}{ Med Auto - High Ped } & Run 1 & 19.6 & 18.3 & \multirow{10}{*}{$3.16 \mathrm{E}^{-08}$} \\
\hline & Run 2 & 19.9 & 18.5 & \\
\hline & Run 3 & 19.9 & 19.0 & \\
\hline & Run 4 & 20.0 & 18.4 & \\
\hline & Run 5 & 19.2 & 18.3 & \\
\hline & Run 6 & 19.7 & 18.8 & \\
\hline & Run 7 & 19.4 & 18.4 & \\
\hline & Run 8 & 20.0 & 18.5 & \\
\hline & Run 9 & 19.8 & 18.5 & \\
\hline & Run 10 & 20.1 & 19.1 & \\
\hline
\end{tabular}


Table A-6 Statistical Comparison of Delays for High Auto Volume Scenarios with Optimized Signal Timing (80s CL) for the Multnomah Street Network

\begin{tabular}{|c|c|c|c|c|}
\hline Scenario & Simulation Runs & Coordinated & Free & p-value \\
\hline \multirow{10}{*}{ High Auto - Low Ped } & Run 1 & 17.0 & 15.6 & \multirow{10}{*}{$5.09 \mathrm{E}^{-09}$} \\
\hline & Run 2 & 17.2 & 15.1 & \\
\hline & Run 3 & 17.4 & 14.5 & \\
\hline & Run 4 & 16.6 & 14.4 & \\
\hline & Run 5 & 17.2 & 14.4 & \\
\hline & Run 6 & 17.4 & 14.0 & \\
\hline & Run 7 & 16.5 & 15.6 & \\
\hline & Run 8 & 17.3 & 14.7 & \\
\hline & Run 9 & 17.3 & 14.5 & \\
\hline & Run 10 & 17.0 & 14.6 & \\
\hline \multirow{10}{*}{ High Auto - Med Ped } & Run 1 & 19.6 & 22.6 & \multirow{10}{*}{0.01} \\
\hline & Run 2 & 19.6 & 21.8 & \\
\hline & Run 3 & 19.4 & 21.7 & \\
\hline & Run 4 & 19.7 & 18.6 & \\
\hline & Run 5 & 19.3 & 20.4 & \\
\hline & Run 6 & 19.5 & 18.9 & \\
\hline & Run 7 & 19.2 & 21.8 & \\
\hline & Run 8 & 19.9 & 19.9 & \\
\hline & Run 9 & 19.2 & 24.5 & \\
\hline & Run 10 & 19.1 & 22.3 & \\
\hline \multirow{10}{*}{ High Auto - High Ped } & Run 1 & 21.1 & 24.1 & \multirow{10}{*}{0.00} \\
\hline & Run 2 & 21.7 & 30.1 & \\
\hline & Run 3 & 21.1 & 26.1 & \\
\hline & Run 4 & 21.7 & 36.1 & \\
\hline & Run 5 & 21.0 & 25.7 & \\
\hline & Run 6 & 21.3 & 36.3 & \\
\hline & Run 7 & 21.2 & 27.0 & \\
\hline & Run 8 & 21.5 & 25.9 & \\
\hline & Run 9 & 21.7 & 24.5 & \\
\hline & Run 10 & 21.4 & 25.4 & \\
\hline
\end{tabular}

